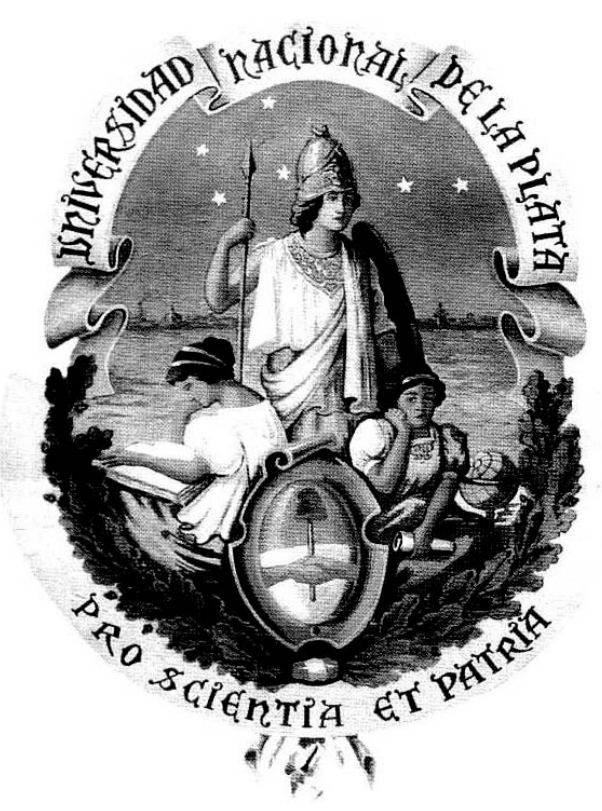

Universidad Nacional de La Plata

Facultad de Ciencias Astronómicas y Geofísicas

Evolución QUíMICA DE GALAXIAS:

RELACIÓN MASA-METALICIDAD Y PERFILES DE ABUNDANCIAS

Lic. Florencia Collacchioni

Directora: Dra. Sofía Alejandra Cora

Codirectora: Dra. Claudia del Pilar Lagos Urbina

Tesis presentada para optar por el grado de

Doctora en Astronomía 


\section{Evolución Química de Galaxias: Relación masa-metalicidad y Perfiles de abundancias}

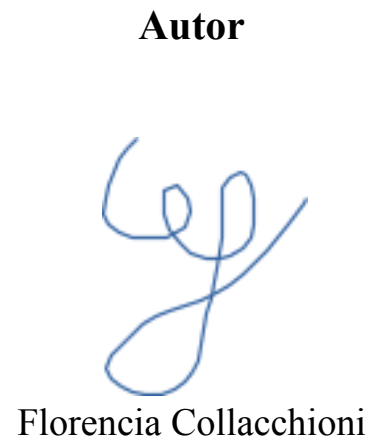

\section{Directores}

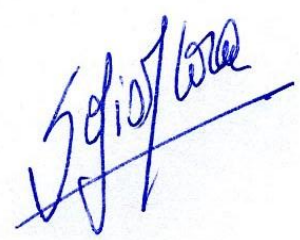

Director/a de tesis

Dra. Sofía Alejandra Cora

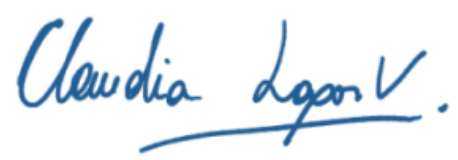

Codirector/a de tesis

Dra. Claudia del Pilar Lagos Urbina

Miembros del jurado: Dr. Guillermo Hägele, Dra. María Emilia De Rossi y Dra. Susana Pedrosa

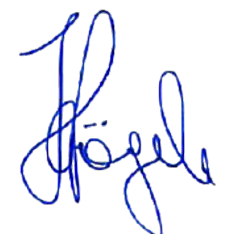

Presidente del Jurado ${ }^{1}$

Guillermo Hägele

\footnotetext{
1 Se debe incluir la firma del presidente del Jurado quien deberá avalar que dicho ejemplar es la versión final de la Tesis defendida, incluyendo las modificaciones menores sugeridas por el Jurado en los casos que corresponda. De forma opcional pueden estar incluidas las firmas de los miembros restantes del jurado.
} 


\section{Resumen}

Durante las últimas décadas, se han realizado numerosos e importantes avances respecto a nuestro entendimiento sobre la formación y evolución de galaxias, tanto desde un enfoque observacional como teórico. En particular, el estudio de la metalicidad (definida a partir de abundancias químicas) de distintas componentes de las galaxias permite entender los diferentes procesos que ocurren en las galaxias durante su evolución, debido a que la misma está relacionada tanto con distintas propiedades galácticas, como la masa estelar, la tasa de formación estelar y el contenido gaseoso, así como con importantes procesos físicos que ocurren en las galaxias. Sin embargo, desde el punto de vista observacional, el estudio de la metalicidad resulta un desafío, ya que existen diferencias entre los resultados arrojados por los distintos métodos utilizados para estimarla y, por lo tanto, surgen discrepancias en la determinación de las propiedades vinculadas. Debido a esto, la comparación con resultados simulados no resulta sencilla, y constantemente se están perfeccionando modelados de procesos físicos para entender cómo, elemento a elemento, las galaxias van enriqueciéndose químicamente.

En esta Tesis, nos enfocamos en estudiar de manera teórica la metalicidad del gas de las galaxias mediante un análisis tanto global como local. Nuestro objetivo es aportar información sobre los procesos que dan lugar a los cambios del enriquecimiento químico durante la evolución del Universo.

En primer lugar, estudiamos la evolución de la relación masa-metalicidad usando el modelo semianalítico de formación y evolución de galaxias SAG. Esta relación muestra que galaxias con mayor masa estelar también presentan mayor cantidad de metales en la fase fría de su componente gaseosa. Sin embargo, se ha encontrado que la forma de esta relación cambia con el tiempo. A partir del análisis del impacto de la retroalimentación energética y química de las supernovas, encontramos que si ésta es mayor a alto corrimiento al rojo es posible explicar la evolución de la relación masa-metalicidad.

En segundo lugar, estudiamos los perfiles radiales de metalicidad utilizando las simulaciones hidrodinámicas cosmológicas EAGLE, las cuales permiten estudiar las galaxias como objetos extendidos (a diferencia de SAG). En este desarrollo, analizamos qué propiedades galácticas afectan la forma que los perfiles presentan, para reconocer distintos procesos físicos que dejan su huella en ellos. Hallamos que la pendiente interna de los perfiles de metalicidad está regulada por la tasa con la cual el gas es acretado por las galaxias.

Finalmente, extendemos el análisis previamente descrito y tomamos regiones de aproximadamente $1 \mathrm{kpc} \times 1 \mathrm{kpc}$ en los discos de las galaxias simuladas por EAGLE para estudiar cómo la metalicidad se ve alterada a escala local por distintos factores, como la densidad superficial de masa estelar y la fracción de gas acretado. Encontramos, así, que la metalicidad local del gas anti-correlaciona con la fracción de gas acretado y que esta relación resulta más fundamental que con otras propiedades.

En toda esta investigación, abordamos, por un lado, un problema clave de los modelos semianalíticos en cuanto a las predicciones de la relación masa-metalicidad y avanzamos en el entendimiento de su evolución. Por otro, desmenuzamos los discos de las galaxias simuladas para analizar en detalle la distribución de metales que resulta del aporte del gas acretado de las galaxias, su dependencia con las regiones en que esto sucede, y los efectos que tiene tal distribución en las propiedades de las galaxias en una escala tanto local como global. De esta manera, este trabajo aporta información clave en el estudio de la evolución de las galaxias. 


\section{Agradecimientos}

Esta Tesis no hubiera sido posible sin el aporte y el apoyo de muchas personas.

Quiero agradecer por todas sus enseñanzas a mis directoras, la Dra. Sofía Cora y la Dra. Claudia Lagos. Más allá de las repreguntadas, las corridas y los husos horarios, siempre estuvieron más que predispuestas a ayudarme a transitar el doctorado, con mucha paciencia y cariño. Trabajar con personas de tal calibre y que además son mujeres es muy importante para mí.

Además de tener directoras hermosas, quiero destacar y agradecer a nuestro grupo. Gracias Tomy, Tuna y Cristian por ayudarme cuando no salía un código o para charlar de algún tema (sea científico o no). También quiero agradecerle a la Dra. Camila Correa, quien fue clave para desarrollar uno de los Capítulos de esta Tesis.

Agradezco a las personas que han sido parte de la Secretaría de Posgrado durante mi tiempo como estudiante de doctorado -Ileana, Milva, María Laura y Consuelo- por la predisposición en solucionar los problemas que me fueron surgiendo. También quiero agradecer a los miembros del Jurado, el Dr. Guillermo Hägele, la Dra. María Emilia De Rossi y la Dra. Susana Pedrosa. Sus comentarios ayudaron a mejorar este trabajo y los intercambios tenidos guiarán mis futuras producciones.

Infinitas gracias a las personas que me ayudaron a seguir en los momentos más estresantes (y en los no tanto también) con un mate, una birrita, un abrazo prepandémico, una charla motivacional o un grito grupal. Les quiero al infinito y más allá.

Gracias a mi fan \#1: mi mamá. A pesar de todavía no terminar de entender del todo mi trabajo, me apoya en todo lo que hago.

Y por supuesto, gracias a Lauti. Sin vos no creo haber podido escribir una tesis en plena pandemia. 


\section{Índice general}

Resumen

Agradecimientos

1. Introducción 1

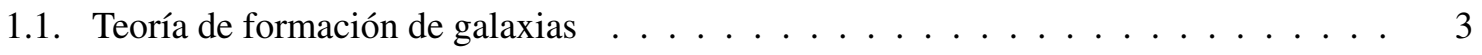

1.1.1. Simulaciones de DM: Método de $N$-cuerpos . . . . . . . . . . . 5

1.1.2. Simulaciones hidrodinámicas . . . . . . . . . . . . 6

1.1.3. Modelos semi-analíticos . . . . . . . . . . . . . . . . 7

1.2. Importancia del enriquecimiento químico en la formación de galaxias . . . . . . . . 7

1.2.1. La relación masa-metalicidad . . . . . . . . . . . . . . . 9

1.2.2. Estudio de los perfiles radiales de abundancia . . . . . . . . . . . . . . 11

1.2.3. Metalicidades en la nueva era . . . . . . . . . . . . . . . 15

1.3. Motivación y estructura de esta Tesis . . . . . . . . . . . . . . . . . . 18

2. Modelos de Formación y Evolución de Galaxias 19

2.1. Modelo semi-analítico SAG . . . . . . . . . . . . . . . . . . 20

2.1.1. Simulación cosmológica MDPL2 . . . . . . . . . . . . . . 20

2.1.2. Características generales del modelo semi-analítico SAG . . . . . . . . . . 21

2.1.3. Modelado de feedback de SNs . . . . . . . . . . . . . . . . . . . . . 24

2.1.4. Modelo de enriquecimiento químico . . . . . . . . . . . . . . . . 25

2.1.5. Calibración del modelo SAG . . . . . . . . . . . . . . . . . . . 27

2.1.6. Definiciones de variantes del modelo . . . . . . . . . . . . . . . . . . . 30

2.2. Simulaciones hidrodinámicas cosmológicas EAGLE . . . . . . . . . . . . 31

2.2.1. Técnica SPH utilizada . . . . . . . . . . . . . . . . . . 32

2.2.2. Modelos físicos de subgrilla implementados en EAGLE . . . . . . . . . . 33

2.2.3. Simulaciones usadas en este trabajo . . . . . . . . . . . . . . . . . 40

2.2.4. Calibraciones de las simulaciones EAGLE y tipos de convergencia . . . . . . 41

3. El impacto del Feedback de SN en la Relación Masa-Metalicidad 46

3.1. La relación masa-metalicidad a $z=0 \ldots \ldots \ldots$. . . . . . . . 46

3.2. Evolución de la Relación Masa-Metalicidad . . . . . . . . . . . . . . . . . . . 48

3.3. El origen de la Evolución de la MZR . . . . . . . . . . . . . . . . . . . 50

3.3.1. Comparación de la MZR predicha por variantes de SAG . . . . . . . . . 50

3.3.2. Evolución de la densidad cósmica del gas frío y la SFR . . . . . . . . . . . . . . . 51

3.3.3. El rol de la dilución de metales en eyecciones de gas . . . . . . . . . . . . 55

3.3.4. La importancia el enriquecimiento químico de la fase caliente del gas . . . . 56

3.4. Conclusiones sobre la evolución de la MZR . . . . . . . . . . . . . . . 58 
4. El efecto de la Tasa de Gas Acretado en los Perfiles Radiales de Abundancia 61

4.1. Muestra de galaxias simuladas . . . . . . . . . . . . . . . . . 61

4.2. Cálculo de la tasa de acreción de gas . . . . . . . . . . . . . . . . . 62

4.3. Estimación de los perfiles radiales de metalicidad del gas . . . . . . . . . . . 63

4.4. La conexión entre la metalicidad, la masa estelar y la $\dot{M}_{\text {accr }} \ldots \ldots \ldots$. . . . . . . . 63

4.5. Efecto de la $\dot{M}_{\text {accr }}$ en los gradientes de metalicidad del gas . . . . . . . . . . . 66

4.5.1. La relación entre RMP y $\dot{M}_{\text {accr }} \ldots \ldots \ldots \ldots$. . . . . . . . . . 66

4.5.2. La relación entre RMP y masa estelar . . . . . . . . . . . . . . . . . . 68

4.5.3. La relación entre SFR, $\dot{M}_{\text {accr }}$ y RMP . . . . . . . . . . . . . . . . . . . . 71

4.5.4. La relación entre RMP y la fracción de gas . . . . . . . . . . . . . . . . 74

4.6. Discusión sobre el impacto de la $\dot{M}_{\text {accr }}$ en los RMP . . . . . . . . . . . . . . . 75

4.7. Conclusiones sobre el estudio de los RMPs . . . . . . . . . . . . . . 78

5. Análisis del contenido químico de regiones locales en galaxias simuladas $\quad \mathbf{8 0}$

5.1. Descripción de regiones locales simuladas y sus propiedades . . . . . . . . . . . 81

5.1.1. Fracción de gas acretado . . . . . . . . . . . . . . . . . . 83

5.2. Conexión entre relaciones resueltas e integradas . . . . . . . . . . . . . . 83

5.2.1. La Relación Masa-Metalicidad resuelta . . . . . . . . . . . . . . . . . . 84

5.2.2. La Secuencia Principal resuelta en galaxias . . . . . . . . . . . . . . . . . . 86

5.3. Fracción de gas acretado como reguladora de la metalicidad . . . . . . . . . . . 89

5.3.1. Análisis de los residuos de la metalicidad . . . . . . . . . . . . . . . . . . 91

5.4. Regiones de acreción de gas en galaxias . . . . . . . . . . . . . . . . . . . . 92

5.5. Conclusiones sobre el estudio de la metalicidad local . . . . . . . . . . . . . . . . 99

$\begin{array}{ll}\text { 6. Conclusiones } & 101\end{array}$

A. Masas de oxígeno en las distintas componentes bariónicas de SAG 114

B. Características de la relación $Z_{\mathrm{hot}}-M_{\star}$ a $z=0$ en SAG 116

B.1. Relación entre $M_{\text {halo }}$ y la dispersión en la relación $Z_{\mathrm{hot}}-M_{\star} \ldots \ldots \ldots \ldots$

B.2. Conversión de $\bar{Z}_{\mathrm{Fe}, 500}-T_{500}$ a $Z_{\mathrm{hot}}-M_{\star} \ldots \ldots \ldots \ldots \ldots$. . . . . . . 117

C. Convergencia de la relación $\dot{M}_{\text {accr }}-$ RMP en EAGLE 118

D. Comparación de la pendiente de los RMP con trabajos anteriores 120

E. Tasa de acreción de gas como función de la masa estelar en EAGLE 122

F. Relación entre la fracción de hidrógeno molecular y la $\dot{M}_{\text {accr }}$ en EAGLE

G. Influencia del tamaño de spaxels en la estimación de propiedades locales de las galaxias 126 


\section{Capítulo 1}

\section{Introducción}

El concepto de galaxias como "universos aislados" data de mediados de Siglo XVIII a través de los trabajos de Wright (1750) y Kant (1755), aunque el estudio de la formación de galaxias no comenzó hasta luego de que Hubble (1929) confirmara su naturaleza extragaláctica. De hecho, muchos de los trabajos iniciales sobre formación y evolución galáctica se llevaban a cabo por la necesidad de responder preguntas de índole cosmológica (como, por ejemplo, si el Universo comenzó con una gran explosión conocida como Big Bang). En este sentido, las galaxias constituyen las unidades fundamentales del Universo, donde las estrellas pueden encontrarse organizadas (Mo et al., 1998; Naab \& Ostriker, 2017). A partir de los descubrimientos de Hubble, el estudio de la formación y evolución de galaxias ha tomado una relevancia importante y crecido por sí mismo.

Las teorías de formación de galaxias consisten en describir los complejos procesos físicos que tienen lugar en las galaxias con el fin de explicar la formación de estas. Dado que las galaxias son observadas a escalas temporales y espaciales cosmológicas, la descripción de su formación y evolución debe involucrar la cosmología y el estudio de las propiedades del espacio-tiempo a gran escala. El modelo cosmológico moderno (o modelo cosmológico estándar) se basa en el principio cosmológico, la hipótesis de que el Universo es homogéneo e isótropo, y en la teoría de la relatividad general de Einstein, de acuerdo a la cual la estructura del espacio-tiempo es determinada por la distribución de masa del Universo. Así, el modelo cosmológico estándar especifica la geometría a gran escala del Universo, y también predice su historia térmica y su contenido de masa y energía. Sin embargo, al suponer un Universo homogéneo e isótropo no es posible explicar la formación de estructuras. Este problema es resuelto al extender la cosmología estándar con la teoría inflacionaria, la cual supone que el Universo temprano experimenta una etapa de rápida expansión exponencial (llamada inflación) debida a la energía de vacío de uno o más campos cuánticos. En muchos modelos inflacionarios, las fluctuaciones cuánticas de esta energía de vacío pueden producir perturbaciones en el campo de densidad de masa con propiedades consistentes a las observadas en la estructura a gran escala (Mo et al., 2010).

El modelo más aceptado para describir el origen y desarrollo de la estructura del Universo en expansión es el llamado modelo $\Lambda \mathrm{CDM}$ (donde $\Lambda$ es la llamada constante cosmológica y CDM se debe a Cold Dark Matter, White \& Rees, 1978; Blumenthal et al., 1984). El mismo puede predecir tanto el ensamble de las estructuras de masa como la historia de formación galáctica. En este modelo, se considera que el Universo consta de tres componentes principales: materia "bariónica" como protones, neutrones y electrones (aunque estos sean leptones y no bariones, en el modelo cosmológico se los incluye al hablar de materia bariónica) que componen el Universo visible, materia oscura y energía oscura (la naturaleza de estas dos últimas es aún desconocida). Varias observaciones revelaron que nuestro Universo es consistente con una geometía plana y la densidad de energía está compuesta en un $\sim 75 \%$ de densidad de energía debido a la constante cosmológica $\Lambda, \sim 21 \%$ debida a la materia oscura fría, y un $4 \%$ restante correspondiente a la materia bariónica de la que las estrellas y galaxias 
están compuestas (Bennett et al., 2013; Planck Collaboration et al., 2020). Dado que el grueso de la masa gravitante en el Universo se encuentra en forma no bariónica (aquélla que no emite radiación y se separa del plasma en expansión antes de la recombinación; Ellis 2000), una galaxia en el Universo es, entonces, material bariónico atraído gravitatoriamente por el pozo de potencial de los halos de materia oscura (DM, por dark matter). Estos halos son el resultado del crecimiento de las fluctuaciones de densidad por inestabilidad gravitacional en los regímenes lineal y cuasi-lineal, seguido del colapso gravitacional de las sobredensidades en el régimen no-lineal y posterior proceso de virialización (Press \& Schechter, 1974). La formación de las galaxias depende del juego entre el enfriamiento del gas y la formación estelar, así como también de las interacciones y fusiones galácticas producto del crecimiento de masa de las estructuras, la cual está gobernada por la fusión jerárquica de los halos de DM.

Dentro de los denominados modelos jerárquicos dominados por CDM hay variantes que difieren en los parámateros cosmológicos asumidos (densidad de materia $\Omega_{\mathrm{m}}$, densidad bariónica $\Omega_{\mathrm{b}}$, densidad de energía oscura $\Omega_{\Lambda}$, constante de Hubble $H_{0}$, etc.), y en las condiciones iniciales, caracterizadas por un espectro de potencias de las fluctuaciones del campo de densidad iniciales con pendiente $n$ ( $n=1$ en la mayoría de los modelos inflacionarios), y normalización absoluta $\sigma_{8}$ (estimada en términos de la variación de las fluctuaciones de masa a escala de $8 h^{-1} \mathrm{Mpc}$ ). Ninguno de estos parámetros clave es conocido con certeza (Ellis, 2000). Los observables más relevantes que, en los últimos años, han sido usados para respaldar alguna de estas variaciones incluyen la normalización absoluta de las fluctuaciones a gran escala del fondo cósmico de microondas (CMB, por cosmic microwave background; Bunn \& White 1997; Planck Collaboration et al. 2020), la densidad numérica local de cúmulos ricos de galaxias (White et al., 1993), el espectro de potencias de la distribución local de galaxias (Peacock, 1997), y supernovas del tipo Ia (SNIa) como brillo estándar (o standard candles, Riess et al. 1998). En términos generales, estos observables no restringen completamente las variantes del modelo $\Lambda \mathrm{CDM}$ si se le permite a los parámetros cosmológicos tener un rango libre. Sin embargo, la evolución de estructuras con corrimiento al rojo (redshift, de aquí en más) entre $0<z<2$, tiempo en que se cree que la población local de galaxias se ha ensamblado, depende sensiblemente de los valores de $\Omega_{\mathrm{m}}$ y $\Omega_{\Lambda}$. Entonces, si resultara posible restringir independientemente estos parámetros, observaciones sistemáticas de galaxias distantes proveerían información con menor ambigüedad sobre los aspectos físicos de la formación de galaxias (Ellis, 2000). La conclusión de esto es que será difícil encontrar un atajo al progreso sustancial en el entendimiento de la formación galáctica dejando de lado los esfuerzos para acotar los parámetros cosmológicos (Ellis, 2000). De acuerdo al último relevamiento de Planck Collaboration et al. (2020), la densidad de energía oscura toma un valor de $\Omega_{\Lambda}=0.685 \pm 0.007$, mientras que la densidad de materia resulta $\Omega_{\mathrm{m}}=0.315 \pm 0.007$.

La astronomía, y en particular el estudio de la formación de las galaxias, es excepcionalmente entorpecida por los sesgos observacionales (Benson, 2010). En este sentido, las simulaciones cosmológicas de formación de galaxias juegan un papel crucial para nuestro entendimiento de la formación galáctica. Esto se debe a que, por un lado, las simulaciones cosmológicas no están limitadas por los intrumentos utilizados en las observaciones y, por el otro, tienen la capacidad de predecir posibles caminos evolutivos, que luego pueden ser contrastados con futuras observaciones. Actualmente, las simulaciones modernas de formación de galaxias reproducen un gran número de resultados observacionales y crean universos virtuales que son, a primer orden, una buena representación del Universo real. Por ejemplo, dado que se cree que la estructura a gran escala del Universo se produjo a través de inestabilidades gravitacionales, la distribución de velocidades peculiares de las galaxias producidas por el campo gravitacional es una relación directa de la distribución de masa subyacente (Mo et al., 2010). Al mismo tiempo, estas simulaciones también son empleadas para explorar modelos cosmológicos alternativos con modificaciones de la naturaleza en la física conocida (como teorías de DM tibia o gravedad modificada; ejemplos Bode et al. 2001; Macciò et al. 2019; Winther et al. 2015; Arnold et al. 2019). Este progreso en el campo de las simulaciones de formación galáctica ha sido 
principalmente motivado por la necesidad de un mejor entendimiento de la física de formación de galaxias, el refinamiento de los métodos numéricos, y el continuo crecimiento del poder de cómputo (Vogelsberger et al., 2020).

A pesar de que las simulaciones cosmológicas de formación de galaxias usan una variedad de diferentes métodos numéricos e implementaciones de la física involucrada, las mismas convergen en un amplio rango de predicciones de la evolución de las galaxias (Somerville \& Davé, 2015; Vogelsberger et al., 2020). Algunas de ellas son la función de masa de halo (Tinker et al., 2008), la distribución de materia oscura a gran escala (Springel et al., 2006), la distribución de hierro en el medio intra cúmulo (Vogelsberger et al., 2018), la longitud de correlación de dos puntos dependiente de la masa (Springel et al., 2018), la formación de discos de las galaxias de tipo tardío (Wetzel et al., 2016), y formación de sistemas de tipo temprano dominados por esferoides (Naab et al., 2014). Entonces, pareciera que los mecanismos físicos básicos que moldean la población de galaxias han sido identificados, y que su actual modelado es suficiente para reproducir poblaciones de galaxias realistas. Sin embargo, estos procesos físicos son implementados a través de modelos de sub-resolución más bien rudimentarios. Los modelos de sub-resolución (también conocidos como modelos de subgrilla) apuntan a capturar la física relevante a través de una descripción eficaz. Estos modelos se construyen a partir de un cierto número de parámetros ajustables y posiblemente degenerados, por lo que la reproducción de grandes cantidades de observables no implica automáticamente un modelo correcto y físicamente posible. Al construir nuevos modelos y simulaciones, es importante recordar que el fin mayor de las simulaciones no es solamente ajustar a los datos observados, sino realizar aportes en el entendimiento de la física de formación de galaxias (Vogelsberger et al., 2020).

Las galaxias resultan un laboratorio complejo, en donde diversos eventos astrofísicos tienen lugar. En particular, el estudio de la evolución química de las galaxias resulta fundamental para poder entender su formación. La cantidad de elementos químicos en el gas de las galaxias puede alterar los tiempos de enfriamiento del gas y de la consecuente formación estelar, y dar indicios de acreción y/o eyección de material por fuera del halo galáctico. El objetivo de esta Tesis es, entonces, realizar un estudio teórico de los cambios en la composición química de las galaxias de acuerdo a los diversos procesos que suceden en ellas, con el fin de esclarecer su rol en la formación y evolución de galaxias. Para ello, estudiamos distintos procesos y relaciones galácticas utilizando diferentes herramientas numéricas. Por un lado, hacemos uso del modelo semi-analítico de formación y evolución de galaxias SAG (Cora et al., 2018, C18 a partir de aquí) para estudiar el impacto producido por los vientos estelares y las explosiones de supernovas (SNs) en la evolución de la relación que vincula la metalicidad del gas con la masa estelar de las galaxias (relación masa-metalicidad). Por el otro, analizamos las salidas de las simulaciones hidrodinámicas EAGLE (Evolution and Assembly of GaLaxies and their Environments, Schaye et al., 2015, S15 a partir de aquí) para poder contar con la distribución espacial de la metalicidad de las galaxias. Así, estudiamos la importancia de la acreción de gas en el proceso de enriquecimiento químico y, en consecuencia, de la evolución de las galaxias.

En lo que resta de este Capítulo introductorio se presenta una descripción resumida de las diferentes herramientas numéricas actualmente existentes para estudiar la formación de galaxias (Sección 1.1), se detalla el contexto actual en el que se encuentra el paradigma de evolución química de galaxias (Sección 1.2), y se presentan las motivaciones de esta Tesis y su estructura (Sección 1.3).

\subsection{Teoría de formación de galaxias}

La materia oscura constituye la espina dorsal de la formación de galaxias, las cuales se esperan que se formen en los centros de las sobredensidades de materia oscura, llamadas halos, debido a la atracción gravitatoria que la materia oscura ejerce sobre la materia bariónica. Se supone que la CDM (componente considerada por los modelos utilizados en esta Tesis) puede ser representada por un fluido no colisional que se desacopla del plasma caliente antes de la época de recombinación cuando 
ya era no-relativista. El límite continuo de la no-interacción de partículas de DM se describe a través de la ecuación de Boltzmann no colisional, acoplada con la ecuación de Poisson. Este par de ecuaciones suele resolverse utilizando una gravedad Newtoniana dado que las estructuras a gran escala no lineales inducen velocidades por debajo de la velocidad de la luz (Mo et al., 2010). La gran dimensionalidad de la ecuación de Boltzmann sin colisiones prohíbe métodos que den soluciones numéricas eficaces basadas en técnicas de discretización estándar para ecuaciones diferenciales parciales. Por consiguiente, en la última década, otras técnicas numéricas han sido desarrolladas para resolver este problema más eficientemente. En especial, en los últimos años se han visto enormes progresos provenientes de dos fuentes: simulaciones de gran volumen modelando grandes muestras de galaxias, y simulaciones "aumentadas" (técnica zoom-in, de aquí en más referidas como simulaciones zoom-in) con modelos refinados de formación de galaxias que resuelven los procesos físicos con mayor detalle (Vogelsberger et al., 2020). Las simulaciones cosmológicas de formación galáctica han sido usadas también para explorar modelos cosmológicos alternativos al CDM. En el nivel más básico, el modelo cosmológico puede ser alterado de tres maneras: formas alternativas de materia oscura, formas alternativas de energía oscura, o formas alternativas de gravedad. Esta área de investigación es muy activa, pero para esta Tesis nos enfocaremos en investigar la formación y evolución de galaxias suponiendo un modelo $\Lambda$ CDM.

Existen, comúnmente, dos tipos de condiciones iniciales aplicadas: (1) volúmenes grande periódicos uniformemente muestreados, y (2) condiciones iniciales zoom, donde hay una alta resolución de la región de interés rodeada por un campo de densidad con baja resolución. La primera se enfoca en estudiar distintas relaciones galácticas de manera estadística, aplicando condiciones de borde periódicas para imitar la homogeneidad e isotropía a gran escala de la distribución de materia en el Universo (es decir, el principio cosmológico). Mientras que la segunda se centra en propiedades detalladas, por lo que las mismas re-simulan con mayor resolución una región o galaxia en particular seleccionada a partir de la simulación de gran escala. El costo computacional de estas simulaciones zoom-in incrementa con la masa del objeto a estudiar a una dada resolución en masa. Por ejemplo, simulaciones zoom-in de galaxias enanas son computacionalmente menos costosas que las simulaciones zoom-in de grandes cúmulos de galaxias debido a la necesidad de utilizar alta resolución en ambos tipos de objetos (Vogelsberger et al., 2020). Algunas simulaciones también emplean condiciones iniciales restringidas para imitar, por ejemplo, el Universo Local (como las sobredensidades de materia oscura cercanas, entre otras; Salmon 1996).

Los modelos de formación de galaxias dependen de un cierto número de parámetros ajustables. Dependiendo de la implementación exacta del modelo, estos parámetros pueden ser elegidos en base a argumentos físicos o pueden requerir un cierto procedimiento de calibración. Este último acercamiento es usualmente utilizado en simulaciones de gran volumen, donde los modelos de subgrilla son menos detallados en comparación con las simulaciones zoom-in. El proceso de calibración consiste en la exploración de un espacio de parámetros involucrados en la descripción de procesos físicos implementados en los modelos a través de un gran número de simulaciones. La calibración está entonces basada en la comparación de los resultados de estas simulaciones con algunos observables clave de la población de galaxias, como la densidad de tasa de formación estelar en función del tiempo cósmico, la función de masa estelar de galaxias a $z=0$ y la relación masa estelar-masa de halo a tiempo presente (ver la reseña de Somerville \& Davé, 2015).

En esta sección, describimos brevemente las técnicas numéricas más usadas para simular poblaciones de galaxias y las que usaremos a lo largo de este trabajo: las simulaciones de materia oscura (Sección 1.1.1), las simulaciones cosmológicas hidrodinámicas (Sección 1.1.2) y los modelos semianalíticos (Sección 1.1.3). El campo de las simulaciones numéricas ha alcanzado un interesante punto donde las simulaciones cosmológicas hidrodinámicas han empezado a reproducir observaciones fundamentales a un nivel parecido al de los modelos semi-analíticos (SAMs, por Semi-Analytic Models). Es interesante notar que gran parte de este éxito se debe a que las simulaciones hidrodinámicas han 
adoptado una aproximación que usan los SAMs: la variación de los parámetros libres que regulan los procesos físicos para poder reproducir ciertos observables, y la experimentación con distintas recetas de subgrilla. Resulta alentador que las dos técnicas han alcanzado conclusiones cualitativamente similares sobre la formación y evolución de galaxias (Somerville \& Davé, 2015).

\subsubsection{Simulaciones de DM: Método de $N$-cuerpos}

En un universo dominado por DM, una suposición común es que los bariones no cambian el comportamiento de la DM y, por lo tanto, las propiedades de los halos de DM pueden ser obtenidas de las simulaciones que sólo modelan la evolución del campo gravitatorio dictado por la DM. En estas simulaciones, los halos de DM son identificados a través de métodos para encontrar grupos gravitatoriamente ligados de partículas de DM como los algoritmos friend-of-friends y extensiones de éstos. En una cosmología que incluye CDM, las estructuras se forman a través de las fusiones jerárquicas de halos de DM (Baugh, 2006), como se muestra en el esquema de la Figura 1.1.

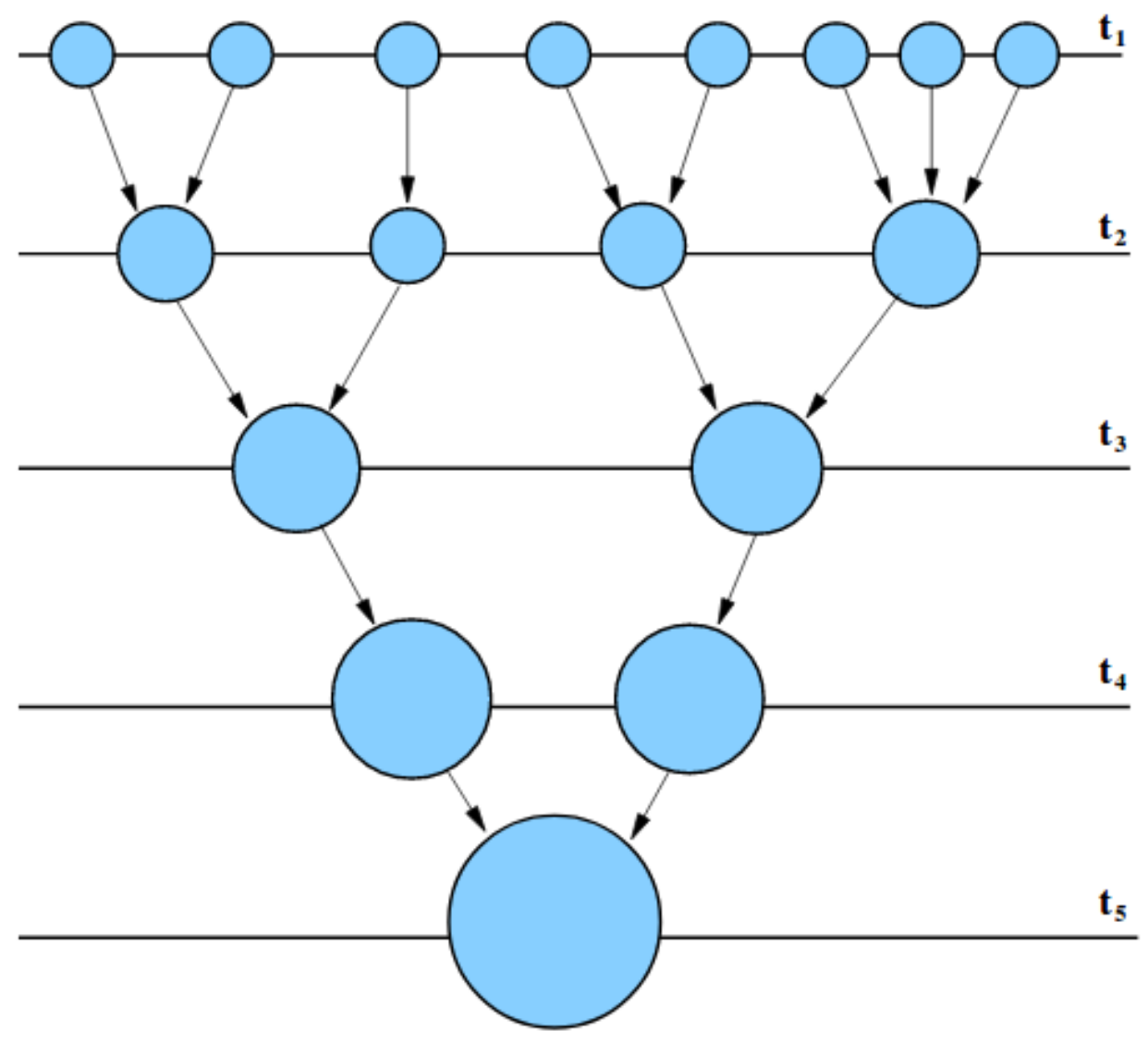

Figura 1.1: Figura 5 de Baugh (2006) que muestra un esquema del árbol de fusión de un halo de materia oscura. Las líneas horizontales representan salidas, snapshots, en la evolución de la historia del halo, correspondientes a distintos pasos de tiempo en una simulación de $N$-cuerpos o a un árbol de fusión realizado con la técnica Monte Carlo $\left(t_{1}<t_{2}\right)$. El tamaño de los círculos indica la masa del halo. Los halos crecen por medio de los eventos de fusión entre halos y por acreción de objetos que se encuentran por debajo de la resolución en masa de halos (halos no resueltos), como se muestra entre los pasos $t_{3}$ y $t_{4}$. El halo final se muestra en $t_{5}$.

Un resultado directo de esto es la función de masa de halo, la cual ha sido estudiada exhaustivamente y resulta una importante prueba de la naturaleza de la DM. La forma que esta función tiene 
a altas masas y su evolución con el tiempo pueden ser usados como restricciones a los parámetros cosmológicos (Eke et al., 1996; Ludlow et al., 2019a; Vogelsberger et al., 2020). Un gran éxito de las simulaciones de CDM es su habilidad para predecir la distribución de la materia a grandes escalas, descrita por la función de correlación de dos puntos (Springel et al., 2006). Además de la habilidad de las simulaciones cosmológicas de CDM en predecir la distribución de la materia a grandes escalas, las mismas también han establecido múltiples características de la distribución de DM resultante del colapso y la virialización de los halos de DM, representados por los perfiles de densidad de Navarro-Frenk-White (NFW, Navarro et al. 1997) y de Einasto (Einasto, 1965). Los halos de DM simulados, entonces, resultan ser prácticamente isótropos en sus regiones internas y son, de cierto modo, dependientes del radio a grandes distancias del centro (Vogelsberger et al., 2020).

\subsubsection{Simulaciones hidrodinámicas}

A pesar de que la distribución de materia en el Universo puede ser comprendida a través de los halos de DM, la componente visible de las galaxias consiste en bariones (principalmente gas y estrellas). La simulación de los procesos físicos que afectan a la componente bariónica es así crucial para realizar predicciones del Universo visible. Inicialmente, la componente bariónica está solamente compuesta por gas, en su mayoría hidrógeno y helio. Eventualmente, parte de este gas se transforma en estrellas durante la formación de estructuras. Los gases astrofísicos son, en las simulaciones cosmológicas, típicamente descritos como gases ideales no viscosos, a los que se les suman términos que representan una viscosidad artificial. Estos gases siguen las ecuaciones de Euler, por lo que pueden ser expresadas en diferentes formas llevando a diferentes esquemas de discretización numérica. La resolución de las ecuaciones hidrodinámicas en las simulaciones cosmológicas es numéricamente demandante debido al gran rango dinámico, a los flujos altamente supersónicos, y a los altos números de Reynolds.

Las ecuaciones hidrodinámicas tienen que ser complementadas por varios procesos astrofísicos, que se espera que sucedan a escalas más pequeñas que la resolución de estas simulaciones y que moldean la población de galaxias. La mayoría de estos procesos son implementados a través de modelos de subgrilla, los cuales son necesarios dada la limitada resolución numérica de las simulaciones. Las simulaciones de la componente de DM típicamente combinan la implementación de métodos eficaces de $N$-cuerpos y esquemas de paralelización. Las simulaciones de la componente bariónica son, sin embargo, más desafiantes debido a que requieren de esquemas numéricos hidrodinámicos confiables y modelos de subgrilla bien planteados.

Las ecuaciones hidrodinámicas pueden ser discretizadas de maneras diversas implementando métodos que robustamente caen en tres clases: técnicas Lagrangianas, Eulerianas o Lagrange-Euler arbitrarias. Las especificaciones Lagrangianas suponen un observador que sigue un fluido individual, con propiedades como la densidad, a medida que se mueve a través del espacio y el tiempo. Las especificaciones Eulerianas, por otro lado, se enfocan en regiones específicas del espacio por donde el fluido fluye al pasar el tiempo. Las técnicas Lagrange-Euler arbitrarias, en cambio, permiten elegir libremente entre las opciones anteriores. Asimismo, las aproximaciones numéricas pueden también distinguirse entre algoritmos de malla libre o malla fija, donde los primeros no requieren conexiones entre los nodos y los segundos se basan en interacciones entre los nodos vecinos.

Las simulaciones cosmológicas tienen que cubrir una gran escala espacial y temporal. Esto implica que las simulaciones tienen que apuntar al mayor número posible de elementos resueltos. Sin embargo, incluso las simulaciones de avanzada no pueden capturar todas las escalas relevantes. Las simulaciones son, entonces, usualmente realizadas a diferentes niveles de resolución para entender la dependencia exacta de los resultados con el número de los elementos de resolución. Así, se dice que la predicción de una simulación es convergente una vez que dicha predicción no cambia significativamente al incrementar la resolución numérica. 


\subsubsection{Modelos semi-analíticos}

El estudio de la física de bariones a través de simulaciones hidrodinámicas es computacionalmente costoso comparado con las simulaciones de $N$-cuerpos de DM. Una aproximación alternativa es modelar la física bariónica mediante la combinación de simulaciones numéricas cosmológicas de DM y SAMs de formación y evolución de galaxias; estos últimos tienen en cuenta los procesos físicos que afectan a la componente bariónica. En este sentido, los SAMs construyen la población de galaxias usando como base la estructura de los halos de DM, los cuales se extraen de una simulación cosmológica de DM, junto con los árboles de fusión de los halos. Este método no resuelve explícitamente las ecuaciones fundamentales que gobiernan la hidrodinámica de un fluido, que puede ser representado por partículas o celdas de grillado, sino que adopta un conjunto de ecuaciones simplificadas que intervienen en el modelado de las distintas componentes bariónicas de las galaxias, tales como disco y halo gaseosos, disco estelar, bulbo estelar (Baugh, 2006; Benson, 2010).

Los modelos semi-analíticos siguen, por ejemplo, la acreción de gas en los halos, el enfriamiento de gas caliente y su conversión en estrellas, o los procesos de retroalimentación (feedback de aquí en más) que remueven gas frío de las galaxias o calientan el halo de gas, entre otros. Un esquema de los diversos procesos físicos que pueden implementarse en los SAMs puede verse en la Figura 1.2.

La ventaja fundamental de los SAMs es que son computacionalmente económicos en comparación con las simulaciones hidrodinámicas (Benson, 2010). Esto facilita la construcción de muestras de galaxias órdenes de magnitud mayores a las posibles en simulaciones hidrodinámicas actuales. Al mismo tiempo, permiten explorar el espacio de parámetros de manera rápida, así como también la física implementada (Henriques et al., 2009; Silk \& Mamon, 2012). Esto permite hacer predicciones para grandes volúmenes, o simular galaxias con diversos rangos de masa de halo, y también permite explorar ampliamente diferentes recetas de subgrilla (Somerville \& Davé, 2015). Es entonces posible desempeñar un amplio rango de cálculos, usando diferentes variantes del modelo con bajo costo computacional, al tiempo que se genera una población de galaxias que puede ser comparada con los datos observacionales de manera similar a los resultados de las simulaciones cosmológicas hidrodinámicas (Vogelsberger et al., 2020). Sin embargo, una desventaja de los SAMs es que son menos auto-consistentes en comparación con las simulaciones hidrodinámicas (Vogelsberger et al., 2020), en el sentido de que no se evoluciona conjuntamente la DM con la materia bariónica. Además, el estudio de las propiedades detalladas del gas (como por ejemplo el gas circumgaláctico) con estos modelos no es directamente posible dado que la componente del gas no se encuentra resuelta.

\subsection{Importancia del enriquecimiento químico en la formación de gala- xias}

Desde hace más de medio siglo se ha estudiado la importancia del contenido químico en la componente gaseosa de las galaxias (McClure \& van den Bergh, 1968; Lequeux et al., 1979). Este, mejor conocido como metalicidad del gas ${ }^{1}$, resulta de gran importancia ya que se cree que está relacionado con diversos procesos de formación y evolución que moldean a las galaxias a través del tiempo. Las estrellas, a lo largo de sus vidas, generan nuevos elementos químicos gracias a la nucleosíntesis estelar. Estos elementos son expulsados al medio que rodea a estas estrellas a través de vientos estelares y, al final de sus vidas, por medio de explosiones de SN. La gran energía que estos procesos liberan calientan el gas que circula por la galaxia. A su vez, el material expulsado contamina este medio, enriqueciéndolo químicamente a medida que pasa el tiempo. Sin embargo, las galaxias no se encuentran aisladas, de forma que interactúan con otras galaxias o incluso con flujos de gas que caen a ellas

\footnotetext{
${ }^{1}$ Debido al hecho que en toda esta Tesis estudiaremos la metalicidad de la componente gaseosa, de aquí en más nos referiremos a la misma simplemente como "metalicidad" (a menos que se estipule lo contrario).
} 


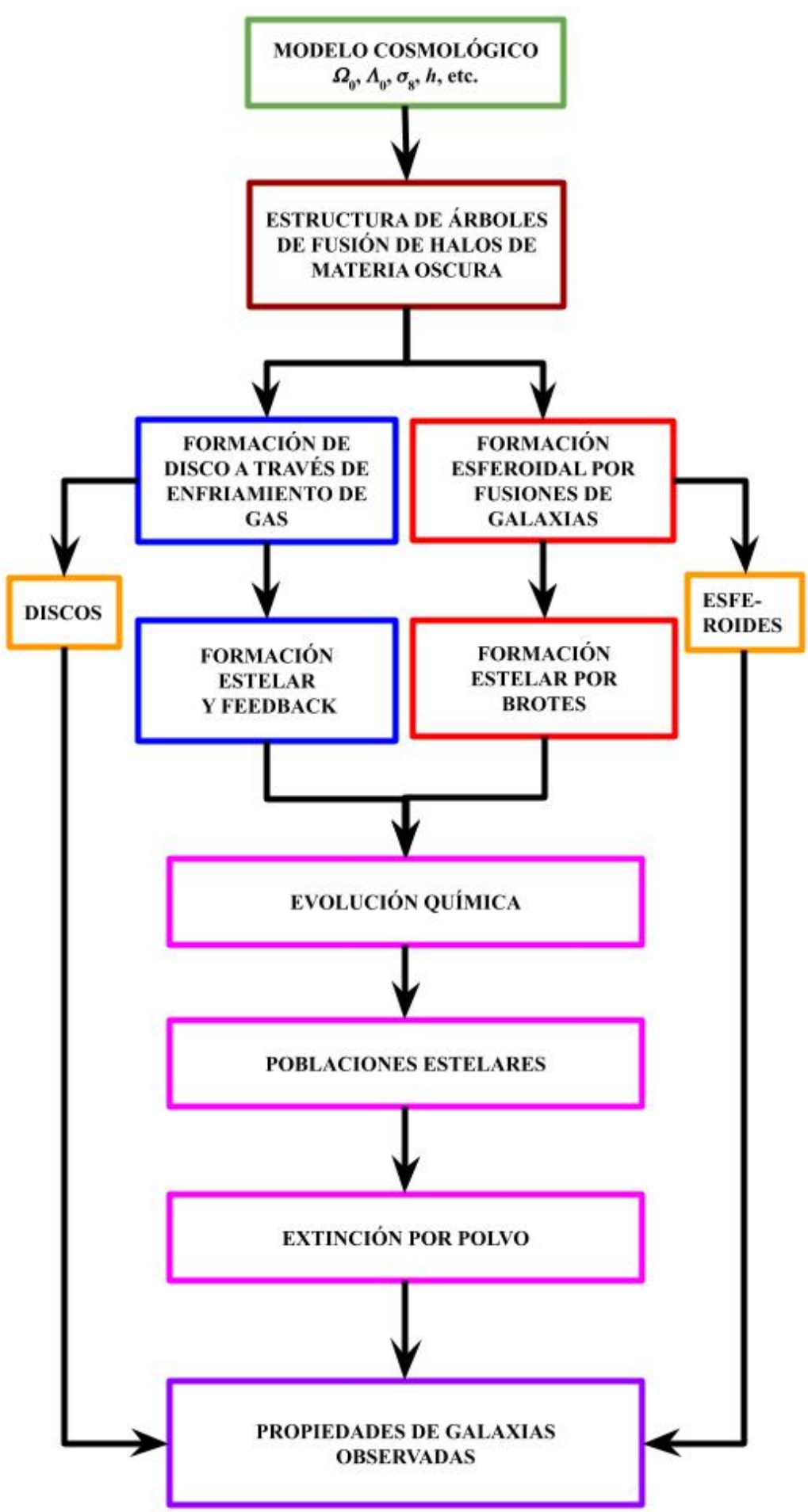

Figura 1.2: Adaptación de la figura 7 de Baugh (2006) que muestra un resumen esquematizado de los ingredientes del modelo jerárquico de formación de galaxias (adoptado de Cole et al. 2000). 
por medio de la gravedad. Este gas puede ser tanto prístino, de composición casi primordial, o previamente enriquecido, alterando los procesos anteriores y complicando el panorama de la evolución química de las galaxias. A su vez, las galaxias son capaces de eyectar material por fuera de su pozo de potencial, resultando éste disponible para que esa misma galaxia lo acrete tiempo después, así como también que lo haga otra galaxia (ver la reseña de Maiolino \& Mannucci 2019).

Todos estos diversos escenarios dificultan una explicación directa de cómo es la evolución de las galaxias, sobre todo cuando hay propiedades que son difíciles de estimar observacionalmente, como la acreción de gas o incluso la metalicidad en sí (Kewley \& Ellison, 2008). En este sentido, poder entender cómo es la evolución del enriquecimiento químico resulta fundamental para poder explicar los distintos caminos de formación y evolución de galaxias.

\subsubsection{La relación masa-metalicidad}

Una relación estadística que describe las propiedades químicas de la población de galaxias es la relación masa-metalicidad (MZR, por Mass Metallicity Relation, donde $Z$ se utiliza para referirse a la metalicidad). La misma correlaciona la masa estelar de las galaxias con la abundancia del oxígeno relativa al hidrógeno de su componente gaseosa, de forma que galaxias con mayor masa estelar también muestran mayor metalicidad (Tremonti et al., 2004), como puede verse en el panel izquierdo de la Figura 1.3. Observacionalmente, la metalicidad de la fase gaseosa es inferida por medio de líneas de emisión a partir del espectro de las galaxias, por lo que estas galaxias tienen una formación estelar activa (Mo et al., 2010). La MZR provee información valiosa sobre el impacto de diferentes procesos físicos que dan lugar a la formación y evolución de galaxias. Al morir, las estrellas producen metales que retornan al medio interestelar (ISM, por Interstellar Medium) a través de la pérdida de masa, enriqueciéndolo al tiempo que se produce una dilución del gas si las caídas de gas puro están presentes (Zahid et al., 2012). Así, esta competencia de mecanismos deja huellas en la abundancia del oxígeno que puede ser usada para inferir acreciones de gas (Zahid et al., 2014b; Lagos et al., 2016). Otro proceso físico importante que puede afectar la metalicidad son las eyecciones galácticas de gas, las cuales pueden transportar parte de sus metales al halo de la galaxia y más lejos (Anglés-Alcázar et al., 2017).

Una característica distintiva de la MZR es que, a masas estelares $\geq 10^{10} \mathrm{M}_{\odot}$ la metalicidad se aplana a valores casi constantes (Tremonti et al., 2004). Este efecto ha sido atribuído al "downsizing" de las galaxias (Maiolino et al., 2008), es decir, galaxias con mayor masa estelar tienen su pico de tasa de formación estelar (SFR, por Star Formation Rate) a redshift más altos. En estos casos, la tasa de contaminación del gas frío en galaxias masivas decae considerablemente comparado con galaxias de baja masa, las cuales todavía forman estrellas e inyectan nuevos metales al ISM. Zahid et al. (2013) argumentan que el aplanamiento puede también ser causado por saturaciones en la metalicidad a una abundancia del oxígeno equivalente a la producida por la nucleosíntesis estelar de las estrellas, que tienen tiempo suficiente para evolucionar y devolver todos esos metales al medio. Estos autores incluso afirman que esta saturación es producto del equilibrio entre los metales inyectados por estrellas al morir (usualmente masivas) y los metales que aún están contenidos en estrellas vivientes (Zahid et al., 2014a).

Muchos autores han encontrado que la MZR evoluciona en su normalización, de modo que a alto redshift las galaxias tienen menor abundancia de oxígeno a masa estelar fija (Erb et al., 2006; Maiolino et al., 2008; Nagao et al., 2008; Mannucci et al., 2009; Møller et al., 2013; Zahid et al., 2014a; Troncoso et al., 2014; Wu et al., 2016; Ly et al., 2016). Se piensa que la evolución de la MZR es una consecuencia de una compleja interacción entre la caída de gas puro hacia las galaxias, las eyecciones de gas rico en metales y la pérdida de masa de las estrellas, como ha sido sugerido de las observaciones de gas atómico y su relación con la metalicidad (Bothwell et al., 2013; Brown et al., 2018).

Otra característica interesante de la MZR es su dispersión observada, incluso a $z=0$, donde 
galaxias de la misma masa estelar presentan un amplio rango de metalicidades ( $1 \sigma$ de \pm 0.3 dex), de acuerdo con la estimación realizada por Mannucci et al. (2010). Estos autores encontraron que introducir la SFR como una variable adicional lleva a una reducción significativa de la dispersión a 0.05 dex, con metalicidades altas asociadas a SFR bajas, a masa estelar fija. El panel derecho de la Figura 1.3 muestra que la dispersión de la MZR puede explicarse al fijar distintos valores de SFR. Ellos fueron guiados por los resultados obtenidos por Ellison et al. (2008) quienes muestran que la MZR tiene una leve correlación negativa con la SFR específica (sSFR, por Specific Star Formation Rate), definida como el cociente entre la SFR y la masa estelar $M_{\star}, \mathrm{SFR} / M_{\star}$, para galaxias con masa estelar de $M_{\star} \leq 10^{10} \mathrm{M}_{\odot}$. Para la misma época, Lara-López et al. (2010) introdujeron el concepto de Plano Fundamental que relaciona masa estelar, metalicidad del gas frío y SFR, el cual pareciera no evolucionar en el tiempo. Esta superficie, a la cual Mannucci et al. (2010) llaman Relación Fundamental de Metalicidad (FMR, por Fundamental Metallicity Relation) es de gran interés porque podría explicar la dispersión de la MZR y proveer información al estudio de galaxias a alto redshift debido a su invarianza temporal (al menos hasta $z \lesssim 2.5$; Mannucci et al. 2010). Sin embargo, el rol de la SFR en la MZR todavía está en debate.
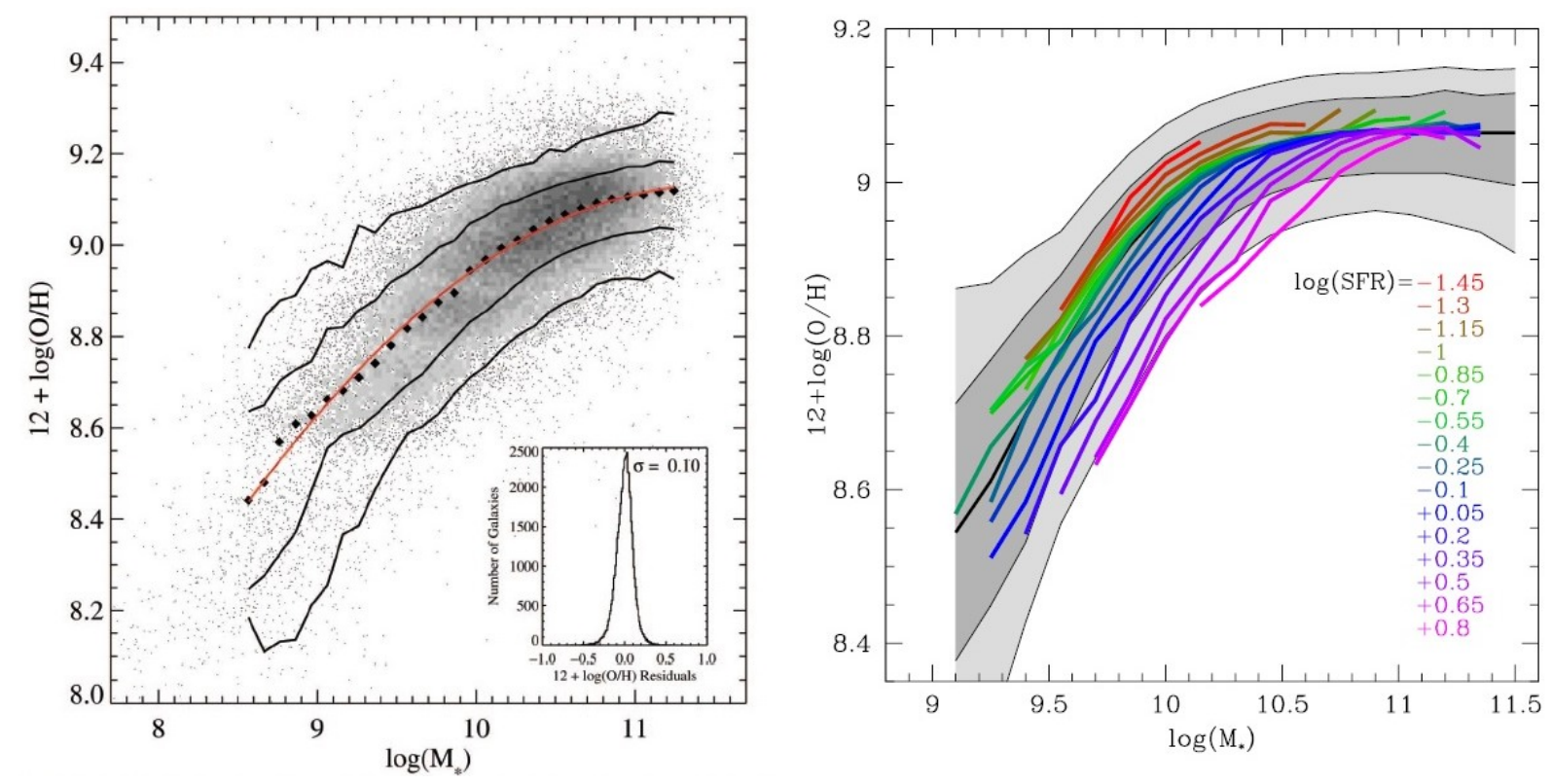

Figura 1.3: Izquierda: Figura 6 de Tremonti et al. (2004), donde se muestra la MZR resultante de $~ 53400$ galaxias del relevamiento Sloan Digital Sky Survey (SDSS). Los diamantes negros representan la mediana en bines de 0.1 dex en masa estelar que incluye al menos 100 puntos. Las líneas sólidas son los contornos que encierran el $60 \%$ y $95 \%$ de la muestra. La línea roja muestra el ajuste polinomial de los datos. El gráfico inmerso muestra los residuos del ajuste. Derecha: Figura 1a de Mannucci et al. (2010), que muestra la MZR para galaxias del relevamiento SDSS. Las áreas grises contienen el 64 y $90 \%$ de todas las galaxias de su muestra observacional, con la línea gruesa central representando la mediana de la relación. Las líneas coloreadas muestran las medianas de las metalicidades, como función de la masa estelar, de galaxias con diferentes valores de tasa de formación estelar.

Sánchez et al. (2013) no encontraron correlación entre la metalicidad y la SFR usando espectroscopía de campo integral, argumentando que la FMR es un producto de la espectroscopía de fibra usada en trabajos previos, la cual sólo da una muestra de las regiones centrales de las galaxias y, por lo tanto, reporta una mirada sesgada de la metalicidad global. Brown et al. (2018) mostraron que, dependiendo de las calibraciones de la metalicidad y la SFR adoptadas, la correlación entre la metalicidad y la SFR puede resultar tanto positiva como negativa. Por otro lado, observaciones del 
hidrógeno atómico $(\mathrm{HI})$ y molecular $\left(\mathrm{H}_{2}\right)$ han sido usadas para sugerir que el contenido de gas puede ser más fundamental que la SFR en determinar la dispersión de la MZR. En la misma línea, Zahid et al. (2014a) usaron un modelo analítico de evolución química para encontrar que la relación entre metalicidad, masa estelar y masa de hidrógeno en gas no evolucionan con el tiempo hasta $z \leq 1.6$. Además, Zahid et al. (2014b) encontraron evidencia observacional de la evolución de la FMR en el mismo rango de redshift. Entonces, ellos concluyeron que la invariancia de la FMR encontrada por Mannucci et al. (2010) es atribuída a los métodos usados para estimar la SFR, y favorecen la idea que la masa de gas en una propiedad más fundamental en la MZR. Bothwell et al. (2013), Hughes et al. (2013) y Brown et al. (2018), entre otros, han mostrado que las metalicidades altas están asociadas con fracciones de gas bajas, a masa estelar fija. Esto pasa naturalmente si la caída de gas juega un papel doble de incrementar la fracción de gas al tiempo que diluye los metales en el ISM (Lara-López et al., 2013).

Intentar reproducir la MZR a bajo y alto redshift ha resultado ser un verdadero desafío para las simulaciones. Modelos semi-analíticos como GALFORM (Cole et al., 2000; Bower et al., 2006; Gonzalez-Perez et al., 2014; Lagos et al., 2012; Lacey et al., 2016) y L-GALAXIES (Springel, 2005; Croton et al., 2006; Guo et al., 2011; Henriques et al., 2015) no reproducen la evolución de la normalización de la MZR encontrada observacionalmente (Guo et al., 2016). Una explicación para este comportamiento puede ser que la metalicidad de las galaxias en SAMs crece a la misma tasa que lo hace la masa estelar (en promedio). No obstante, usando el modelo semi-analítico GAEA (GAlaxy Evolution and Assembly), Xie et al. (2017) confirmaron una evolución en la normalización de la MZR hasta $z \approx 0.7$ para todas las masas estelares que analizaron, y una evolución hasta $z \approx 2$ para la mayoría de las galaxias masivas $\left(M_{\star} \gtrsim 10^{10} \mathrm{M}_{\odot}\right)$. El modelo de eyección y la ley de formación estelar implementados en GAEA fueron considerados como responsables de este resultado, el cual no había sido posible conseguir hasta el momento por otros SAMs.

Las simulaciones cosmológicas hidrodinámicas recientes han tenido más éxito al reproducir la MZR y su evolución. Davé et al. (2011) mostraron que, al cambiar el modelo de vientos estelares en su código de Hidrodinámica de Partículas Suavizada (SPH, por Smoothed Particle Hydrodynamics), pueden encontrar diferentes tasas de evolución de MZR debido al impacto que éste tiene en el juego entre la caída y la eyección de gas. Más recientemente, Davé et al. (2017) mostraron que su nueva simulación cosmológica hidrodinámica MUFASA muestra una MZR cuya normalización evoluciona en 0.4 dex desde $z=0$ hasta $z=6$. Otras simulaciones cosmológicas hidrodinámicas como EAGLE (S15; Crain et al. 2015) muestran una evolución de magnitud similar (Guo et al., 2016; De Rossi et al., 2017). Aún más, Lagos et al. (2016) y De Rossi et al. (2017) mostraron con EAGLE que la metalicidad del gas correlaciona de manera más fundamental con la fracción de gas que con la SFR. Las simulaciones cosmológicas zoom-in FIRE (Feedback In Realistic Environments, Hopkins 2013) también han sido capaces de reproducir la evolución de la normalización de la MZR (Ma et al., 2016), mientras que las discrepancias con las observaciones prevalecen con respecto de la pendiente de la MZR.

\subsubsection{Estudio de los perfiles radiales de abundancia}

La metalicidad total de una galaxia, entendida como la que se estima hasta un determinado radio respecto del su centro galáctico, engloba diversas regiones de una galaxia en donde varios procesos se dan en simultáneo. Para poder tener mejor detalle de los cambios que las galaxias padecen a lo largo de su evolución química, se han utilizado diversas técnicas para estudiar la metalicidad en todo el disco galáctico, como por ejemplo la espectroscopía de rendija larga o long-slit (Díaz et al. 2007; Hägele 2008; Rosa et al. 2014). Estos métodos tienen la ventaja de tener una importante resolución espacial y espectral, aunque se encuentran límitados en cuanto a tiempo de obtención y cantidad de datos que se pueden obtener. En este sentido, surge la necesidad de poder generar grandes muestras de objetos, las cuales presenten homogeneidad en su obtención y tengan una amplia 
cobertura de tipos y regiones de galaxias. Así, y gracias a los avances tecnológicos de los últimos años, nace la espectroscopía de campo integral (IFS, por Integral Field Spectroscopy), utilizada en los relevamientos CALIFA (Calar Alto Legacy Integral Field Area; Sánchez et al., 2012) y SDSSIV MaNGA (Mapping Nearby Galaxies at Apache Point Observator; Bundy et al. 2015), y en los instrumentos KMOS (K-band Multi-Object Spectrograph; Stott et al., 2014), SAMI (Sydney-AAO Multi-objectIntegral-field spectrograp; Bryant et al., 2015; Poetrodjojo et al., 2018) y MUSE (Multi Unit Spectroscopic Explorer; Carton et al., 2018; Erroz-Ferrer et al., 2019). Dado el tipo de estudio realizado en esta Tesis y las ventajas que la técnica de IFS aporta, de aquí en más nos limitaremos a comparar con trabajos que han utilizado este tipo de técnica.

En la última década, la IFS ha permitido avanzar enormemente en el estudio de la MZR al proveer información espacialmente resuelta de la abundancia de metales en un diverso grupo de galaxias, abriendo una nueva ventana al estudio de la importancia del enriquecimiento químico en la evolución de las galaxias. En este sentido, el estudio de los perfiles de metalicidad radial (RMP, por Radial Metallicity Profiles) puede ayudar a entender el papel que el enriquecimiento químico juega en la evolución de las galaxias. Con RMP nos referimos a la abundancia de metales en la componente gaseosa de las galaxias como función de la distancia al centro de las galaxias. Esta abundancia es típicamente inferida a través del análisis de las líneas de emisión nebular (ver Maiolino \& Mannucci 2019 para una reseña reciente). Este perfil es usualmente descrito como $\log _{10}\left(Z / Z_{\odot}\right)=\alpha r+b$, donde $Z / Z_{\odot}$ es la metalicidad en unidades de la metalicidad solar, $\alpha$ es la pendiente del RMP y $b$ su punto cero.

Los estudios pioneros en el tema hallaron que galaxias a bajo redshift $(z \leq 0.5)$ muestran un gradiente negativo del RMP $(\alpha<0)$. Esto significa que las galaxias están más enriquecidas químicamente en sus regiones centrales que en las afueras (Sánchez et al., 2012, 2013; Ho et al., 2015), como puede verse en la Figura 1.4. Este comportamiento es generalmente interpretado como un escenario de formación de adentro hacia afuera (o inside-out, de aquí en más), en donde las estrellas en el centro de las galaxias se forman antes que aquéllas en las regiones externas y, así, tienen más tiempo para enriquecer el ISM, naturalmente produciendo mayor metalicidad de gas en el centro comparado con las zonas externas (Boissier \& Prantzos, 1999). Sin embargo, estudios posteriores han encontrado una variedad de RMPs que complican el panorama. Por ejemplo, Troncoso et al. (2014), basados en el proyecto AMAZE (Assessing the Mass-Abundance red-shift [Z] Evolution), encontraron que galaxias con redshifts en el rango de $3 \leq z \leq 4$ muestran RMPs positivos $(\alpha>0)$, que los autores interpretan como producidos por un flujo de gas de baja metalicidad que cae directo a los centros de las galaxias (ver también Cresci et al. 2010).

En lugar de medir un único $\alpha$ para todo el RMP, Sánchez et al. (2014) y Sánchez-Menguiano et al. (2016) usando el relevamiento CALIFA, y Belfiore et al. (2017) usando el relevamiento SDSSIV MaNGA, además de otros, ajustaron dos leyes de potencia a los RMPs. Dicho de otra manera, encontraron dos valores de $\alpha$, uno para la región interna y otro para la externa, hallando resultados que no pueden ser fácilmente interpretados con un escenario de formación inside-out. Entre estos resultados aparece un aplanamiento de los perfiles en las regiones externas $(\alpha \approx 0)$, o perfiles internos relativamente planos (al menos, menos negativos de lo esperado) en galaxias de alta masa $\left(M_{\star} \gtrsim\right.$ $10^{10} \mathrm{M}_{\odot}$, Sánchez-Menguiano et al. 2016). Esto podría estar relacionado a la remoción preferencial de metales por feedback en las regiones internas de las galaxias (por ejemplo, Lagos et al. 2013; Muratov et al. 2017), así como también una formación estelar significativa en las regiones externas de la galaxia (por ejemplo, debido a fusiones o interacciones cercanas) que pueden rápidamente enriquecer el gas. 

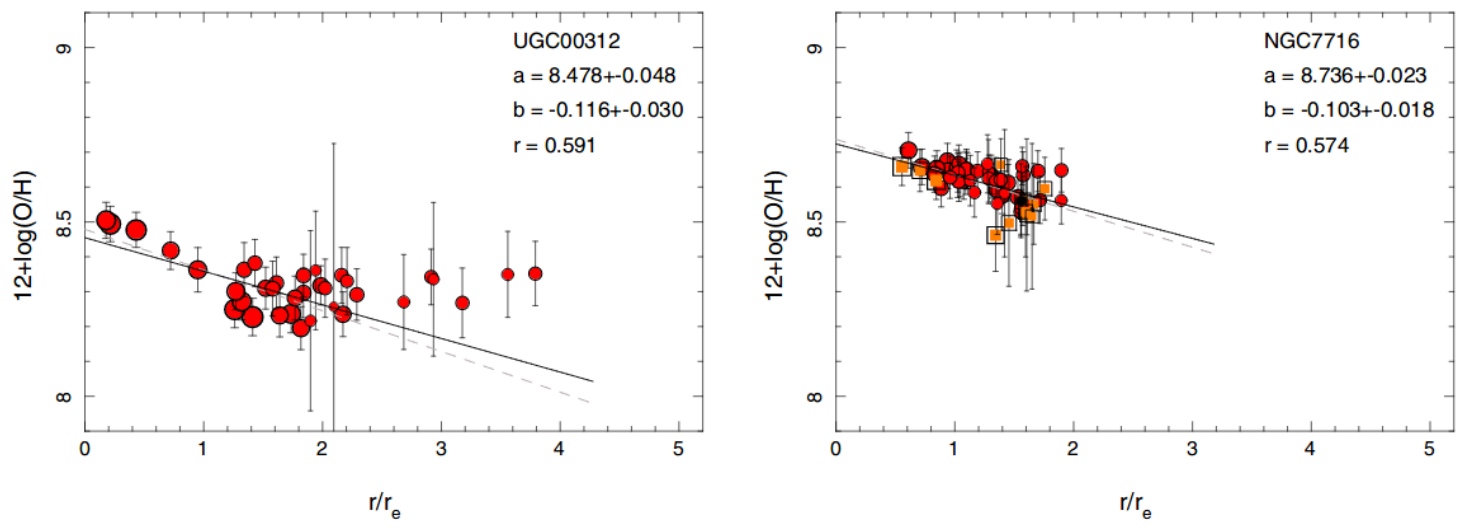

Figura 1.4: Figura 5 de Sánchez et al. (2014), en donde se muestra la distribución radial de la abundancia del oxígeno derivada a partir de observaciones de regiones H II individuales, con errores en la abundancia por debajo de 0.15 dex, como función de la distancia galactocéntrica proyectada (es decir, corregida por inclinación), normalizada al radio efectivo del disco. El tamaño de los símbolos es proporcional a la intensidad de la línea $\mathrm{H}_{\alpha}$. Los círculos rojos representan aquellas regiones $\mathrm{H}$ II donde las líneas de emisión son producto de la formación estelar, mientras que los cuadrados naranjas representan aquellas regiones cuyas líneas de emisión provienen de una mezcla de fuentes de ionización. Las líneas sólidas y a trazos muestran el mejor ajuste lineal y un ajuste lineal pesado con los errores entre los valores de radio 0.3 y $2.1 r_{\mathrm{e}}$. Los resultados del segundo ajuste son mostrados en la figura, incluyendo el punto cero (a), la pendiente (b), y el coeficiente de correlación (r).

Varios estudios han explorado las posibles causas físicas detrás de la forma de los RMPs. De acuerdo con Sánchez et al. (2012), el cambio en las regiones externas (más allá de $\approx 2$ veces el radio efectivo) podrían ser debidos a diferentes densidades en el gas, a la historia de formación estelar, o incluso a la presencia de barras que pueden alterar el flujo de gas interno de las galaxias. Aún así, Zinchenko et al. (2019), usando el relevamiento CALIFA, no encontraron correlación entre la pendiente del RMP y barras o patrones espirales, y Carton et al. (2018) no encontraron correlación entre la pendiente $\alpha$ y la SFR o con las masas estelares de las galaxias cuando analizaron los datos de MUSE. Esto se encuentra en tensión con los reportes de una débil correlación entre $\alpha$ y la sSFR por Stott et al. (2014) y Wuyts et al. (2016) usando los relevamientos KMOS, y con la masa estelar por Ho et al. (2015) quienes usaron muestras de galaxias combinadas de CALIFA y SDSS Data Release (DR) 7. Más aún, Ho et al. (2015) encontraron que al mostrar el RMP normalizado con el radio efectivo de la galaxia se obtiene una correlación entre $\alpha$ y las propiedades de las galaxias, implicando una co-evolución del gas y de las estrellas.

Es justo decir que la tensión entre los diferentes resultados observacionales, que pueden ser en parte debido a las diferentes selecciones de galaxias, puede también sugerir que el RMP no está bien descrito por una sola o doble ley de potencia, y/o que otras propiedades de galaxias pueden correlacionar más fuertemente con el RMP que la sSFR, la masa estelar y la morfología. De hecho, Carton et al. (2015), midiendo el contenido gaseoso del hidrógeno atómico, HI, y usando mediciones de 50 galaxias del SDSS observadas con el radiotelescopio WSRT (Westerbork Synthesis Radio Telescope), encontraron que las pendientes del RMP muestran una fuerte correlación con la fracción de masa de $\mathrm{HI}, \mathrm{HI} / M_{\star}$, de forma tal que las galaxias con mayor fracción de HI también tienen pendiente $\alpha$ más negativa.

Desde una perspectiva teórica, se ha reportado un abanico de resultados provenientes de simulaciones hidrodinámicas de formación de galaxias, los cuales no necesariamente están de acuerdo entre sí. Tissera et al. (2016), usando un volumen cúbico simulado de $14 \mathrm{Mpc}$ de lado a partir de una versión del código hidrodinámico P-GADGET-3, argumentaron que un rango de pendientes, desde muy negativas hasta positivas, puede resultar debido a encuentros cercanos de galaxias, presencia de barras y/o acreción de gas de baja metalicidad en las regiones centrales de las galaxias. Esta variedad 
de resultados son causados por los procesos que desencadenan la actividad de formación estelar y la eyección de gas producto de SNs. Un resultado similar es reportado por Sillero et al. (2017), quienes también utilizaron simulaciones hidrodinámicas provenientes de una versión del código P-GADGET3 , y concluyeron que las interacciones de galaxias generalmente llevan a $\alpha>0$ para los casos de fusiones mayores de galaxias de disco, pero detectan variaciones significativas en la distribución de metalicidad y pendientes durante el proceso de fusión. Además, Sillero et al. (2017) encontraron que la fracción inicial de gas de una fusión de galaxias y la fuerza de la feedback por SNs tienen un efecto en $\alpha$.

Una limitación importante de los estudios mencionados es la pobre estadística debido a los pequeños volúmenes simulados, lo que dificulta la identificación de procesos principales que llevan a valores de $\alpha$ más altos/bajos. Las nuevas generaciones de simulaciones de galaxias han permitido una explicación más rigurosa de estas tendencias con mejores estadísticas gracias a un mayor tamaño de las cajas cosmológicas simuladas. Un ejemplo de esto son las simulaciones EAGLE (S15; Crain et al. 2015); su mayor caja tiene unos $100 \mathrm{Mpc}$ de lado, $\approx 360$ veces mayor volumen que los estudios antes mencionados. Usando EAGLE, Tissera et al. (2019) mostraron que las galaxias que han padecido recientemente numerosas fusiones tienen asociadas RMPs planos, lo que naturalmente lleva a galaxias de baja masa teniendo RMPs más negativos. Tissera et al. (2016) y Sillero et al. (2017) concuerdan en que galaxias con masa estelar de $M_{\star} \leq 10^{10} \mathrm{M}_{\odot}$ muestran RMPs más negativos que las galaxias masivas. Otras simulaciones, sin embargo, encuentran resultados contradictorios. Ma et al. (2017), usando simulaciones cosmológicas zoom del proyecto FIRE (Hopkins et al., 2014), reportaron que galaxias de baja masa tienen RMPs más planos comparadas con galaxias masivas (cabe aclarar que estas simulaciones sufren de un bajo número estadístico). Tissera et al. (2019) tampoco encontraron correlación entre $\alpha$ y el ambiente en donde las galaxias viven, mientras que el tamaño físico de los discos gaseosos de las galaxias aparece como una de las únicas propiedades de galaxias que claramente correlacionan con $\alpha$. Es interesante notar que Tissera et al. (2016) encontraron que, a medida que el redshift aumenta, las galaxias de alta masa $\left(M_{\star} \gtrsim 10^{10} \mathrm{M}_{\odot}\right)$ muestran valores de $\alpha$ menos negativos e incluso, a veces, valores positivos.

En este contexto, es esperable que la acreción de gas moldee tanto la historia de formación estelar como la historia de enriquecimiento químico de las galaxias y, entonces, se podría explorar directamente la correlación entre el RMP y la tasa de acreción de gas $\left(\dot{M}_{\text {accr }}\right)$. Sin embargo, $\dot{M}_{\text {accr }}$ no es accesible fácilmente a través de observaciones, motivando así a los trabajos basados en simulaciones para explorar estos indicadores indirectos, tales como la formación estelar y la masa estelar. En este sentido, la primera resulta una indicadora dado que se espera que sea proporcional al suministro de gas, de forma que la caída de gas pueda impulsar la formación estelar (por ejemplo, Davé et al. 2011; Lilly et al. 2013), mientras que la segunda se relaciona debido a que las galaxias más masivas viven en halos más masivos (Behroozi et al., 2013b) y, entonces deberían también atraer más gas a su pozo de potencial gravitatorio. Al estudiar directamente el efecto del suministro de gas en las galaxias, Perez et al. (2011) encontraron que pendientes más negativas están asociadas con acreción de gas de baja metalicidad, mientras que Sillero et al. (2017) predijeron una correlación entre $\alpha$ y la sSFR sólo en casos donde el gas que cae desencadena formación estelar en las regiones centrales en escalas de tiempo cortas. El escenario propuesto es que una considerable $\dot{M}_{\text {accr }}$ en las regiones centrales de las galaxias puede hacer crecer la densidad del gas, impulsando altos niveles de formación estelar que rápidamente enriquecen las regiones internas, llevando a pendientes más negativas del RMP. Otros estudios sugieren que una acreción extrema (es decir, fusiones) lleva a gradientes de metalicidad invertidos (Troncoso et al., 2014). Entonces, el estudio de los RMPs puede ser esencial para delimitar las propiedades del gas que está siendo acretado a la galaxia (es decir, gas puro o previamente enriquecido), y el efecto que la $\dot{M}_{\text {accr }}$ tiene en la evolución química de las galaxias (Finlator, 2017). 


\subsubsection{Metalicidades en la nueva era}

Para estimar la metalicidad total de una galaxia, se recurre al análisis de las regiones centrales o medición de espectros usando distintas aperturas (Barrera-Ballesteros et al., 2018). Sin embargo, nos podríamos preguntar si esto alcanza para poder explicar la evolución química de las galaxias. Si en lugar de ver a las galaxias como un todo pudiéramos dividirlas en regiones, esto nos ayudaría a identificar más fácil y rápido si hay alguna acreción o eyección de material restringido a una zona en particular y estudiar la influencia que esto tiene en las galaxias en general. En esta línea, podríamos analizar las relaciones globales que ya conocemos en una escala local. Así, estudiar si estas relaciones locales se condicen con las globales, si se mantienen (o no) a través del tiempo, o sus relaciones entre sí genera un valioso aporte al entendimiento de la evolución química de las galaxias y a la evolución de éstas.

El estudio de los perfiles radiales de metalicidad es una muy buena primera aproximación a responder estos interrogantes. Sin embargo, tiene un efecto homogeneizante de la metalicidad al no tener en cuenta las posibles asimetrías de las galaxias, donde usualmente se supone simetría axial para galaxias con formación estelar activa (o SF, por Star Forming). Asimismo, un incremento (o disminución) en la metalicidad o la SFR en una dirección en particular, y no sólo en función de la distancia al centro de la galaxia, resulta más esclarecedor en cuanto a los procesos físicos involucrados en esos cambios. En este sentido, gracias a los últimos avances en las técnicas de IFS resulta posible realizar este tipo de observaciones, en donde se pueden obtener espectros de distintas regiones de un gran número de galaxias y rearmarlas casi en su totalidad. Estas regiones suelen ser denominadas spaxels (producto de la combinación entre las palabras espectro en inglés, spectrum, y pixel), y la información que aportan de las distintas propiedades galácticas permite construir relaciones resueltas o locales ${ }^{2}$

Los primeros en realizar un estudio de la MZR resuelta o local (rMZR, por resolved MassMetallicity Relation) utilizando IFS fueron Rosales-Ortega et al. (2012). Ellos realizaron espectroscopía óptica de regiones HII espacialmente resueltas de galaxias espirales del Universo cercano utilizando los relevamientos PINGS (PPak Integral Field Spectroscopy Nearby Galaxies Surve; RosalesOrtega et al., 2010) y CALIFA, obteniendo una muestra total de 38 galaxias y 1896 regiones de $\sim 1$ kpc. Encontraron que la metalicidad del gas crece con la densidad superficial de masa estelar $\left(\Sigma_{\star}\right)$ de manera similar a como lo hacen las propiedades integradas, incluso encontrando un aplanamiento de la relación local a medida que crecen los valores de metalicidad para valores altos de $\Sigma_{\star}$. Un ejemplo de esta relación puede verse en el panel izquierdo de la Figura 1.5 (obtenida del trabajo de Hwang et al. 2019). Esto llevó a que estos autores concluyeran principalmente dos cosas. Por un lado, la MZR resulta ser una relación integrada de las propiedades locales. Siguiendo este razonamiento, el origen de la MZR global puede ser explicado como el efecto combinado entre la existencia de la rMZR y un sesgo en las aperturas de las mediciones. Por otro lado, el hecho de que la metalicidad incremente con $\Sigma_{\star}$ refleja la existencia de un gradiente radial de metalicidad, dado que los valores más altos de $\Sigma_{\star}$ se localizan en las regiones centrales, lo que implicaría que la metalicidad disminuye con el radio galactocéntrico (y estaría en acuerdo con un escenario de formación inside-out). Además, analizaron la dependencia de la metalicidad y la $\Sigma_{\star}$ con el ancho equivalente de $\mathrm{H}_{\alpha}$, el cual permite estimar la SFR. Así, la existencia de la FMR en galaxias podría ser explicada por la presencia de una relación intrínseca entre la $\Sigma_{\star}$, la metalicidad y la SFR a nivel local.

Posteriormente, surgieron varios trabajos en donde se aumentaron el número de muestras, se estudiaron diferentes propiedades locales e incluso se analizaron galaxias a alto redshift. Analizando $5 \times 10^{6}$ spaxels SF provenientes de 653 galaxias de disco, Barrera-Ballesteros et al. (2016) encontraron nuevamente que la metalicidad crece con la $\Sigma_{\star}$ y concluyeron en que la rMZR puede reproducir no

\footnotetext{
${ }^{2}$ Llamamos relaciones resueltas o locales a las relaciones que surgen a partir de las propiedades físicas de los spaxels. De aquí en más, nos referiremos al término local como al estudio de los spaxels de las galaxias, los cuales pueden tener tamaños de hasta 0.5 segundos de arco en el Universo Local, como es el caso de la muestra que utilizan Hwang et al. (2019) a partir del relevamiento MaNGA.
} 
sólo la MZR global, sino también los perfiles radiales de metalicidad de sus galaxias observadas. A su vez, afirman que el origen de la MZR y de los RMPs es el mismo y que, entonces, el estudio de la rMZR resulta fundamental al poder dar información de dos relaciones globales tan importantes. Además, encontraron que la rMZR resulta independiente de la masa total y la sSFR de la galaxia (excepto a baja masa estelar y alta sSFR). Así, las propiedades locales jugarían un rol principal en la determinación de las propiedades integradas y, por consiguiente, de las relaciones globales en general. Pilyugin et al. (2017) construyeron mapas de abundancia del oxígeno de los discos de 134 galaxias espirales usando espectroscopía 2D del DR3 del relevamiento CALIFA y examinaron la existencia de un quiebre en la pendiente de los RMPs aplicando dos ajustes a los spaxels analizados, uno lineal puro y otro con una línea quebrada (panel derecho de la Figura 1.5). Encontraron una diferencia entre la pendiente quebrada y la lineal menor al 0.05 dex, sugiriendo que una simple regresión lineal es adecuada, al menos a primer orden, para describir la distribución radial de las abundancias del oxígeno en los discos de galaxias espirales. Por su parte, Sánchez Almeida et al. (2018) estudiaron 14 galaxias enanas del Universo Local y presentaron evidencia de una anti-correlación local entre la metalicidad y la densidad superficial de SFR, $\Sigma_{\text {SFR }}$ (obtenidas mediante el índice $\mathrm{N}_{2}{ }^{3}$ y el flujo de $\mathrm{H}_{\alpha}$ ) que imita la anti-correlación global reportada por Mannucci et al. (2010) y Lara-López et al. (2010), y está en contraposición a Barrera-Ballesteros et al. (2016). Mostraron, además, que esta anti-correlación se mantiene incluso si se eliminan las variaciones a gran escala. Es decir, esta anticorrelación estaría regulada por las variaciones locales de las propiedades, en particular de la caída local de gas pobre en metales la cual alimenta la formación estelar.

Usando observaciones realizadas con IFS de casi $10^{6}$ spaxels de 1023 galaxias del relevamiento MaNGA, Barrera-Ballesteros et al. (2018) hallaron que la fracción de gas local juega un papel principal en el enriquecimiento químico local y, en segundo nivel, en la velocidad de escape local. También concluyen que la fracción de gas local y la metalicidad del gas ionizado son resultados de la evolución galáctica ocurriendo a escalas locales, así como también subrayan el impacto de eyecciones de gas locales que dan forma a la distribución de metalicidad interna de galaxias SF. Sin embargo, y en contraposición a los trabajos mencionados, estos autores afirman que el grado de formación estelar local no juega un papel significativo en predecir la metalicidad, por lo que toda correlación entre la metalicidad y la formación estelar es inducida por la mutua correlación entre la fracción de gas local con la formación estelar y la metalicidad. En esta misma línea, Hwang et al. (2019) analizaron $\sim 5 \times 10^{5}$ spaxels de 1222 galaxias SF de MaNGA y encontraron que el $25 \%$ de ellos presenta una metalicidad sorprendentemente menor en comparación a lo que predice la rMZR, a los que llamaron ALM (por anomalously low metallicity). Para poder explicar esto, los autores propusieron que una acreción de gas prístino en estas regiones es la responsable de la disminución en metalicidad. A su vez, argumentan que las regiones de baja metalicidad trazan los sitios de reciente acreción de gas, la cual estimula la formación estelar en proceso. Más aún, encuentran que el número de estas regiones ALM son mayores en galaxias de baja masa, aportando a la idea que la caída local de gas pobre en metales produce una distribución no-homogénea del material químico, a la vez que tiene un mayor efecto en galaxias de baja metalicidad y masa estelar. Por su parte, Sánchez-Menguiano et al. (2019), usando una muestra de 736 galaxias espirales del relevamiento MaNGA $(z<0.05)$, encontraron que el $60 \%$ de sus spaxels muestran una anti-correlación entre la metalicidad y la $\Sigma_{\mathrm{SFR}}$, mientras que el $21 \%$ presenta una correlación positiva de estas propiedades, lo que implica diversos orígenes. Asimismo, concluyeron que la abundancia característica del oxígeno de una galaxia determina sin ambigüedad la forma de la relación local entre la $\Sigma_{\mathrm{SFR}}$ y la metalicidad. Así, la metalicidad del gas global de una galaxia sería el principal factor en determinar la relación local entre la SFR y la metalicidad.

\footnotetext{
${ }^{3}$ El índice $\mathrm{N}_{2}$ se define como el cociente entre la línea de emisión intensa del Nitrógeno una vez ionizado (NII), ubicada a $\lambda=6583 \AA$ a $z=0$, y la línea de emisión de $\mathrm{H}_{\alpha}$, es decir, $\log \left([\mathrm{NII}] \lambda 6583 / \mathrm{H}_{\alpha}\right)$.
} 

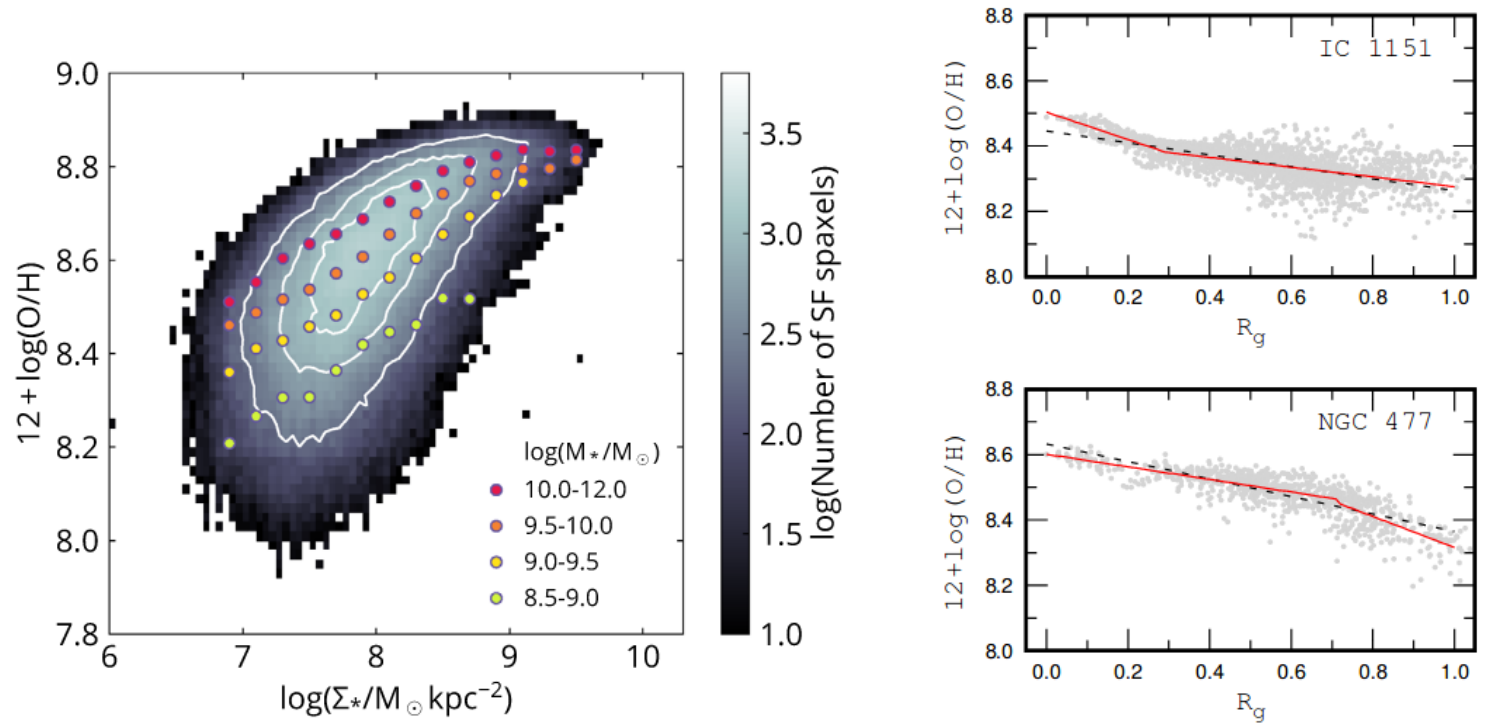

Figura 1.5: Izquierda: Figura 3 de Hwang et al. (2019), donde se muestra la relación local entre la metalicidad y la $\Sigma_{\star}$. Los símbolos representan los picos de distribución de metalicidad en cada rango de masa estelar. La distribución de fondo y los contornos muestran la distribución de todos los spaxels SF puros de galaxias de tipo tardío con masa estelar $>10^{8.5} \mathrm{M}_{\odot}$. Derecha: Ejemplos de la distribución de abundancias en los discos de dos galaxias espirales observadas por Pilyugin et al. (2017) y mostradas en su figura 3 . $\mathrm{R}_{\mathrm{g}}$ es la distancia galactocéntrica normalizada con el radio $\mathrm{R}_{25}$, es decir, el radio isofotal óptico de la galaxia. Los puntos grises representan las abundancias individuales de cada spaxel. La línea negra a trazos muestra un ajuste lineal puro a los datos, mientras que la línea roja sólida representa un ajuste lineal quebrado.

Desde el punto de vista de las simulaciones hidrodinámicas, no hay muchos trabajos que hayan estudiado en detalle la rMZR y las propiedades involucradas con ella. Un ejemplo de tal estudio es el de Trayford \& Schaye (2019), quienes analizan galaxias provenientes de las simulaciones EAGLE aplicando una técnica computacional de suavizado de las propiedades de las partículas, generando regiones similares a los spaxels de $\approx 1 \mathrm{kpc}$ (lo cual resulta por encima del límite de resolución de las simulaciones). Los autores analizan las rMZR y la relación local entre la $\Sigma_{\mathrm{SFR}}$ y $\Sigma_{\star}$, encontrando un buen acuerdo con las observaciones. Además, hacen un estudio de los residuos de estas propiedades locales y encuentran que para valores de $\Sigma_{\star} \lesssim 10^{2} \mathrm{M}_{\odot} \mathrm{pc}^{-2}$, los residuos de la rMZR y los residuos de la relación $\Sigma_{\mathrm{SFR}}-\Sigma_{\star}$ anti-correlacionan, mientras que para valores de $\Sigma_{\star} \gtrsim 10^{2} \mathrm{M}_{\odot} \mathrm{pc}^{-2}$ ocurre lo opuesto. Estos resultados se contraponen con los de Patrício et al. (2019), quienes hallaron que la relación entre estos residuos resulta positiva y dependiente del redshift. A su vez, Trayford \& Schaye (2019) encontraron que el corte de $\Sigma_{\star}$ en la relación de los residuos resulta del cambio de régimen donde predomina el feedback de SN o de Núcleos Galácticos Activos (AGN, por Active Galactic Nuclei). También afirman que la independencia con el redshift de las relaciones entre la metalicidad y la fracción de gas global en las galaxias EAGLE no se preserva a escalas del kpc, implicando que la evolución química es no-local debido al transporte de gas y metales entre las galaxias. Con todo esto, concluyen que, a pesar que la existencia de relaciones locales indican que los procesos físicos (como formación estelar, enriquecimiento químico y feedback) dependen de y operan a escalas locales, las relaciones locales no resultan ser más "fundamentales". En este sentido, la evolución de las galaxias dependería tanto de procesos tanto locales como globales.

Dado que las propiedades de las galaxias simuladas surgen de cálculos hidrodinámicos locales, estudiar las relaciones locales resulta de sumo interés. Debido a que es posible que las simulaciones reproduzcan las propiedades integradas de las galaxias al tiempo que fallan en reproducir las propiedades locales (Crain et al., 2015), las relaciones locales observadas son útiles para diagnosticar 
tanto la estructura como la demografía de las poblaciones de galaxias simuladas. Si las simulaciones pueden reproducir simultáneamente las observaciones de manera global y local, entonces pueden dar información muy valiosa sobre el origen físico de la evolución de las galaxias.

Si bien la metalicidad del gas a nivel global y local promete proveer información crucial sobre la formación y evolución de las galaxias, las observaciones han arrojado conclusiones muchas veces contradictorias. Sin embargo, hay un entendimiento cualitativo general de que la metalicidad del gas debiera estar trazando el efecto conjunto de la acreción de gas, formación estelar y feedback energético de las estrellas masivas y AGNs. Las simulaciones ofrecen una oportunidad ideal para explorar de forma cuantitativa los aspectos antes mencionados, explorando también diversas formas en que se podrían observar tales relaciones.

\subsection{Motivación y estructura de esta Tesis}

En este trabajo, nos proponemos investigar la historia de ensamble de las galaxias a partir del análisis de la forma de la MZR, así como también estudiar la distribución espacial de los metales en el gas de las galaxias, haciendo uso de las técnicas numéricas mencionadas en la Sección 1.1, con el fin de responder algunos de los interrogantes vigentes hoy en día. Por un lado, los SAMs han tenido poco éxito en poder reproducir la evolución de la MZR. La forma en que los procesos físicos relacionados con la metalicidad del gas son modelados en los SAMs es clave en las predicciones de la evolución química. Por otro lado, hay un gran debate en torno a la propiedad o propiedades que determinan la forma de los RMPs. Dado que estos perfiles están sumamente vinculados con diversos procesos físicos de las galaxias, poder entender la razón de sus cambios resulta imperativo para profundizar en el entendimiento de la formación de galaxias. Asimismo, dar aportes en el estudio de la metalicidad resuelta del gas de galaxias simuladas, algo que recientemente ha comenzado a explotarse, resulta importante para entender si los procesos locales modelados por las simulaciones producen efectos similares a los observados y, al mismo tiempo, predecir resultados a alto redshift donde las observaciones son escasas.

Esta Tesis está organizada de la siguiente forma. En el Capítulo 2 se realiza una descripción de las herramientas numéricas utilizadas en este trabajo: el modelo semi-analítico de formación y evolución de galaxias SAG (Sección 2.1) y las simulaciones cosmológicas hidrodinámicas del proyecto EAGLE (Sección 2.2). Mostramos nuestros resultados del estudio de la relación masa-metalicidad de las galaxias modeladas por SAG en el Capítulo 3. Los resultados obtenidos del estudio de los perfiles radiales de abundancia y la importancia de la acreción de gas son incluidos en el Capítulo 4. Los resultados del análisis realizado mediante la utilización de perfiles en 2 dimensiones se describen en el Capítulo 5. Por último, en el Capítulo 6 se presentan las conclusiones de esta Tesis, sentando las bases para los próximos trabajos a futuro. 


\section{Capítulo 2}

\section{Modelos de Formación y Evolución de Galaxias}

Para poder hacer un estudio del enriquecimiento químico de las galaxias, en este trabajo haremos uso de dos herramientas numéricas distintas: modelos semi-analíticos (SAMs) de formación y evolución de galaxias, y simulaciones hidrodinámicas cosmológicas; su descripción general se presentó en la Sección 1.1.

Los SAMs tienen la ventaja de poder producir una población de galaxias lo suficientemente grande como para que los análisis realizados cuenten con una buena estadística, al tiempo que los costos computacionales son bajos. Gracias a su gran versatilidad para poder modificar el modelado de los procesos físicos que afectan a la componente bariónica, estos modelos resultan de gran utilidad para poder estudiar los procesos físicos involucrados en la formación y evolución de las galaxias. Los SAMs pueden combinarse con simulaciones numéricas cosmológicas de distinto tipo y tamaño con la ventaja de que las simulaciones cosmológicas de $N$-cuerpos que sirven de base no deben ser repetidas cada vez que se cambian las suposiciones sobre los procesos bariónicos, las cuales son tenidas en cuenta en los SAMs.

A pesar de que la información que podemos obtener a partir de los resultados de los SAMs es sumamente valiosa, la mayoría no cuenta con una descripción de la distribución espacial de las propiedades de las galaxias como para poder analizar qué le sucede a la metalicidad de éstas a distintas distancias respecto de sus centros. Incluso en los casos en donde se implementa alguna estrategia para modelar el perfil radial de cantidades como masa, metalicidad y momento angular (Stevens et al., 2016; Henriques et al., 2020), se requieren muchas aproximaciones, lo que limita el poder predictivo de estas herramientas. Afortunadamente, en las últimas décadas se han comenzado a desarrollar simulaciones hidrodinámicas que sí permiten trazar esta distribución espacial y no requieren de suposiciones como conservación de momento angular o simetría axial. Estas simulaciones cosmológicas, a diferencia de los SAMs, siguen la evolución tanto de las partículas de materia oscura como de las partículas de gas y estrellas. Si bien las simulaciones hidrodinámicas modelan volúmenes cosmológicos que son de al menos un orden de magnitud menor que aquéllos en los que se basan los SAMs, el nivel de resolución en masa alcanzado por las mismas y/o una mejora de la resolución espacial (regulada por los valores del parámetro de ablandamiento de las ecuaciones dinámicas) permiten realizar un análisis riguroso de las propiedades de las galaxias, con una estadística lo suficientemente buena como para entender los procesos que afectan a las galaxias a lo largo del tiempo. Debido a esto, las simulaciones cosmológicas hidrodinámicas resultan un laboratorio ideal para el estudio de los perfiles radiales de metalicidad, los cuales dependen de la distribución del gas en las galaxias para poder analizarlos.

En este trabajo, entonces, haremos uso del SAM de formación y evolución de galaxias SAG (por Semi-Analytic Galaxies, C18) y de las simulaciones hidrodinámicas cosmológicas EAGLE (por 
Evolution and Assembly of GaLaxies and their Environments, S15). SAG, descrito en la Sección 2.1, ha sido considerablemente mejorado, alcanzando un gran nivel de versatilidad para ser aplicado en distinto tipo de simulaciones cosmológicas, según los requerimientos del problema a estudiar. Por otro lado, las simulaciones EAGLE (Sección 2.2) modelan variados volúmenes cosmológicos con distintas resoluciones espaciales y de masa.

\subsection{Modelo semi-analítico SAG}

La versión actual del modelo semi-analítico SAG es un posterior desarrollo del modelo descrito por Cora (2006), el cual está basado en el modelo presentado por Springel et al. (2001), y luego mejorado según se describe en Lagos et al. (2008), Padilla et al. (2014), Gargiulo et al. (2015), Muñoz Arancibia et al. (2015) y C18. El modelo SAG usa halos de DM extraídos de una simulación cosmológica de DM y sus correspondientes árboles de fusión como entrada para construir la población de galaxias. Consideramos la simulación MULTIDARK MDPL2, que es parte de la base de datos de acceso público CosmoSim ${ }^{1}$. A continuación, presentamos los detalles de la simulación numérica utilizada y describimos brevemente las principales características del modelo SAG haciendo énfasis en los aspectos más relevantes para esta tesis.

\subsubsection{Simulación cosmológica MDPL2}

La simulación cosmológica MDPL2 sigue la evolución de $3840^{3}$ partículas DM en una caja cuyo lado tiene una longitud de $1 h^{-1}$ Gpc. Tiene una resolución de masa de $m_{\mathrm{p}}=1.5 \times 10^{9} \mathrm{~h}^{-1} \mathrm{M}_{\odot}$ por partícula de DM. Esta simulación es análoga a la simulación MDPL descrita en Klypin et al. (2016), pero fue corrida con diferentes condiciones iniciales. Es consistente con un modelo de materia oscura fría $\Lambda$ CDM caracterizado por una cosmología de Planck, cuyos parámetros son: densidad de materia $\Omega_{\mathrm{m}}=0.307$, densidad de energía oscura $\Omega_{\Lambda}=0.693$, densidad bariónica $\Omega_{\mathrm{B}}=0.048$, índice espectral $n_{\mathrm{s}}=0.96$, y constante de Hubble $H_{0}=100 h^{-1} \mathrm{~km} \mathrm{~s}^{-1} \mathrm{Mpc}^{-1}$, con $h=0.678$ (Planck Collaboration et al., 2014b).

Los halos de DM han sido identificados usando el identificador de halo ROCKSTAR (Behroozi et al., 2013b), y los árboles de fusión (o merger trees) fueron construidos con el algoritmo consisTENTTREes (Behroozi et al., 2013c). Para detectar los halos de DM, se consideraron sobredensidades que contuvieran al menos un número de partículas mínimo $N_{\min }=20$. Cada halo es caracterizado por sus propiedades físicas definidas por la distribución de partículas, suponiendo una aproximación de sobredensidad esférica. La masa virial es definida como la masa encerrada en una esfera de radio virial $r_{\text {vir }}$, tal que la densidad media alcance un factor constante de $\Delta=200$ veces la densidad crítica del Universo $\rho_{\mathrm{c}}$ (densidad en la que el Universo tiene geometría plana), es decir,

$$
\begin{aligned}
M_{\mathrm{vir}}\left(<r_{\mathrm{vir}}\right) & =\Delta \rho_{\mathrm{c}} \frac{4 \pi}{3} r_{\mathrm{vir}}^{3}, \\
\operatorname{con} \rho_{\mathrm{c}} & =\frac{3 H^{2}}{8 \pi G},
\end{aligned}
$$

donde $H$ es la constante de Hubble y $G$ es la constante gravitatoria. Más aún, la velocidad virial de cada halo es definida en términos de estas propiedades como $V_{\mathrm{vir}}=\sqrt{G M_{\mathrm{vir}} / r_{\mathrm{vir}}}$. Los halos de DM que son detectados pueden yacer por encima de la densidad de fondo, o encima de otro halo de DM. A partir de ahora, para diferenciarlos, los primeros serán referidos como los halos huéspedes ${ }^{2}$, mientras que los segundos se denominarán subhalos. El cálculo de las propiedades físicas de los subhalos considera sólo las partículas que componen la subestructura detectada por el identificador de halos.

\footnotetext{
${ }^{1}$ http://dx.doi.org/10.17876/cosmosim/mdpl2/007

${ }^{2} \mathrm{El}$ término de halo huésped, provenientes del inglés host halo, también puede ser encontrado en la bibliografía con el nombre de halo anfitrión.
} 
La simulación evoluciona desde redshift $z=17$ hasta la época actual $(z=0)$, almacenando 126 salidas (o snapshots) igualmente espaciadas en $\log (a)$ (siendo $a$ el parámetro de expansión).

\subsubsection{Características generales del modelo semi-analítico SAG}

El modelo SAG incluye los efectos de enfriamiento por radiación del gas caliente, formación estelar, retroalimentación energética por explosiones de supernovas (de aquí en más, feedback de $S N s$ ), seguimiento de varios elementos químicos producidos por diferentes fuentes (vientos estelares y supernovas de Tipo Ia y II) teniendo en cuenta la vida de los progenitores (Cora, 2006). También se ha incorporado el modelado del crecimiento de los agujeros negros (BHs, por Black Holes) supermasivos en los centros de las galaxias, su consecuente retroalimentaicón energética por AGN (feedback de $A G N$, de aquí en más), y brotes de formación estelar desencadenados por inestabilidades de disco o fusiones de galaxias (Lagos et al., 2008). Estos brotes de formación estelar contribuyen a la formación de los bulbos, cuyos tamaños son estimados como se describe en Muñoz Arancibia et al. (2015). El gas frío que ha sido transferido al bulbo es consumido gradualmente, de manera tal que los brotes de formación estelar son caracterizados por una escala de tiempo en lugar de ser instantáneos (Gargiulo et al., 2015). Tecce et al. (2010) agregó el efecto de la presión de barrido (o RPS, por Ram Pressure Stripping) sobre el disco de gas frío de las galaxias satélites, implementando el criterio de Gunn \& Gott (1972).

El modelo SAG sigue la evolución de las diferentes componentes bariónicas de las galaxias. Por un lado, las galaxias cuentan con un halo de gas caliente. El mismo resulta de gas calentado por choques a medida que cae hacia el pozo de potencial gravitatorio del halo de DM. Este halo de gas caliente alcanza una distribución espacial en equilibrio hidrostático evitando colapsar gravitatoriamente hacia el centro del halo de DM debido a su propia presión. Por otro lado, parte del gas del halo caliente puede posteriormente enfriarse, a través de diversos procesos de enfriamiento radiativo, y formar un disco gaseoso. La tasa en la que el gas se enfría depende de la temperatura del gas (la cual determina el estado de ionización), la composición química del gas, y la densidad del gas (esta última determina la tasa con la que las colisiones entre electrones e iones tienen lugar). A medida que el gas se enfría, la presión del gas decae y esto propicia la caída del gas hacia el centro del halo con la escala de tiempo dinámica del halo, conservando el momento angular. Así, el gas frío forma un disco, el cual es soportado por rotación. Por último, a partir de este disco de gas frío se pueden formar estrellas. Dependiendo de su momento angular, las estrellas pueden pertenecer al disco estelar o al bulbo. El primero es aquél formado por estrellas que mantienen el momento angular del disco de gas frío en el cual se formaron. El segundo está compuesto por estrellas cuyo momento angular es significativamente bajo y se localiza en los centros de las galaxias; esto sucede gracias a inestabilidades de disco y a interacciones de galaxias, las cuales modifican el momento angular del gas que forma estas estrellas.

Para describir el halo de gas caliente, se supone que el mismo tiene siempre una distribución similar a la del halo de DM huésped principal, cuya masa virial, $M_{\mathrm{vir}}$, cambia entre salidas consecutivas de la simulación de fondo como resultado del crecimiento jerárquico de la estructura. Inicialmente, la masa del gas caliente está dada por $M_{\text {hot }}=f_{\mathrm{b}} M_{\text {vir }}$, donde $f_{\mathrm{b}}=0.1569$ es la fracción bariónica universal (Planck Collaboration et al., 2014b). Con esta definición, modelamos la acreción cosmológica gradual que tiene lugar en los halos huéspedes principales a medida que su masa virial incrementa. Se supone que el gas acretado tiene una composición química primordial ( $76 \%$ de hidrógeno y $24 \%$ de helio). Una vez que una fracción de gas caliente se enfría, y la formación estelar y el feedback entran en juego, la masa del gas caliente disponible para enfriarse y afluir hacia la galaxia central de cada 
halo huésped principal es calculada como

$$
\begin{aligned}
M_{\mathrm{hot}}= & f_{\mathrm{b}} M_{\mathrm{vir}}-M_{\star, \mathrm{cen}}-M_{\mathrm{cold}, \mathrm{cen}}-M_{\mathrm{BH}, \mathrm{cen}} \\
& -\sum_{i=1}^{N_{\mathrm{sat}}}\left(M_{\star, i}+M_{\mathrm{cold}, i}+M_{\mathrm{BH}, i}+M_{\mathrm{hot}, i}\right),
\end{aligned}
$$

donde $M_{\star}, M_{\text {cold }}$ y $M_{\mathrm{BH}}$ son, respectivamente, las masas totales de estrellas, gas frío y BHs centrales de cada galaxia contenida en un dado halo huésped principal, ya sea ésta central o alguna de las $N_{\text {sat }}$ satélites. Al considerar galaxias satélites, también se descuentan sus respectivas masas de gas caliente.

Una galaxia forma estrellas siempre y cuando su masa de gas frío exceda un valor de masa crítico $M_{\text {cold, crit }}$, con una tasa definida de acuerdo a la implementación descrita en Croton et al. (2006) como

$$
\frac{d M_{\star}}{d t}=\alpha \frac{M_{\mathrm{cold}}-M_{\mathrm{cold}, \text { crit }}}{t_{\mathrm{dyn}}}
$$

donde $\alpha$ es la eficiencia de formación estelar (un parámetro libre del modelo), $t_{\mathrm{dyn}}=0.1 R_{\mathrm{disc}} / V_{\mathrm{vir}}$ es el tiempo dinámico de una galaxia. En esta última expresión, $V_{\text {vir }}$ es la velocidad virial y $R_{\text {disc }}$ es la escala de tamaño del disco dada por $R_{\text {disc }}=(\lambda / \sqrt{2}) r_{\text {vir }}$, donde $\lambda$ es el parámetro de rotación (o spin) del halo huésped (Mo et al., 1998). Para una dada masa de estrellas formadas, $\Delta M_{\star}$, la fracción de masa estelar dentro de un dado rango de masa se calcula suponiendo una función inicial de masa (IMF, por Initial Mass Function), $\Phi(\mathrm{M})$. SAG considera una IMF de Chabrier (Chabrier, 2003), la cual debe ser normalizada por el intervalo de masas estelares, es decir

$$
\int_{M_{\mathrm{L}}}^{M_{\mathrm{U}}} \Phi(M) M \mathrm{~d} M=1
$$

donde $M_{\mathrm{L}}$ y $M_{\mathrm{U}}$ son los límites de masas inferior y superior, respectivamente. Dado que las estrellas que aportan la mayor contribución al enriquecimiento químico son las que tienen $M_{\star} \geq 1 \mathrm{M}_{\odot}$, es necesario fijar la fracción $\xi$ de masa estelar total distribuida en estrellas por encima de $1 \mathrm{M}_{\odot}$. Esto es equivalente a fijar el límite inferior $M_{\mathrm{L}}$ (Portinari et al., 1998), obteniendo así la condición normalizada

$$
\int_{M_{1}}^{M_{\mathrm{U}}} \Phi(M) M \mathrm{~d} M=\xi
$$

donde $M_{1}=1 \mathrm{M}_{\odot}$ y el límite superior se elije como $M_{\mathrm{U}}=100 \mathrm{M}_{\odot}$. Con el fin de alcanzar compatibilidad con la relación edad-metallicitdad observada de las estrellas, se adopta $\xi=0.5$ (Portinari et al., 1998). SAG sigue la pérdida de masa de estrellas de masa baja, intermedia y alta que tiene lugar a través de vientos estelares y explosiones de SNs. En este último caso, el tiempo de vida de los progenitores de SN es considerado, relajando entonces la aproximación de reciclado instantáneo (Cora, 2006).

El feedback de AGN reduce el enfriamiento del gas en halos grandes, previniendo que estos formen estrellas en tiempos tardíos. Este proceso se desencadena debido a los eventos de acreción de gas en los BHs supermasivos. El modelado de feedback de AGN ha sido incluido en SAG por Lagos et al. (2008) siguiendo el trabajo de Croton et al. (2006), aunque el modelado del crecimiento de los BHs fue posteriormente modificado (Ruiz et al. 2015, C18). Los BHs crecen por medio de flujos de gas hacia el centro galáctico, los cuales se desencadenan por medio de inestabilidades de disco o fusiones de galaxias. Se supone que los BHs se fusionan instantáneamente cuando una fusión ocurre. El BH resultante crece a través de la acreción de gas frío siguiendo

$$
\Delta M_{\mathrm{BH}}=f_{\mathrm{BH}} \frac{M_{\mathrm{sat}}}{M_{\mathrm{cen}}} \frac{M_{\text {cold, sat }}+M_{\text {cold, cen }}}{\left(1+280 \mathrm{~km} \mathrm{~s}^{-1} / V_{\mathrm{vir}}\right)^{2}},
$$


donde $M_{\text {cen }}$ y $M_{\text {sat }}$ son las masas de las galaxias central y satélite que se fusionan, y $M_{\text {cold, cen } \mathrm{y}}$ $M_{\text {cold, sat }}$ son sus correspondientes masas de gas frío. La fracción de gas frío acretado por el BH central supermasivo, $f_{\mathrm{BH}}$, es un parámetro libre del modelo. El crecimiento del $\mathrm{BH}$ por inestabilidades de disco también está representado por las ecuación anterior, donde el cociente $M_{\text {sat }} / M_{\text {cen }}$ es reemplazado por la unidad y $M_{\text {cold,sat }}=0$, ya que este proceso depende de las propiedades de una sola galaxia. Los BHs también pueden crecer durante los procesos de enfriamiento de gas que tienen lugar una vez que el halo de gas caliente estático se forma alrededor de la galaxia central. Siguiendo a Henriques et al. (2015), la tasa de crecimiento de BH está dada por

$$
\dot{M}_{\mathrm{BH}}=\kappa_{\mathrm{AGN}} \frac{M_{\mathrm{BH}}}{10^{8} \mathrm{M}_{\odot}} \frac{M_{\mathrm{hot}}}{10^{11} \mathrm{M}_{\odot}},
$$

donde $M_{\text {hot }}$ y $M_{\mathrm{BH}}$ son las masas del halo de gas y del $\mathrm{BH}$, respectivamente. La eficiencia de la acreción de gas caliente sometido a enfriamiento radiativo está dada por el parámetro libre $\kappa_{\mathrm{AGN}}$. El feedback de AGN reduce la cantidad de gas que se enfría mediante

$$
\dot{M}_{\text {cool }}^{\prime}=\dot{M}_{\text {cool }}-\frac{L_{\mathrm{BH}}}{V_{\mathrm{vir}}^{2} / 2},
$$

donde la luminosidad del $\mathrm{BH}, L_{\mathrm{BH}}$, es decir, el calentamiento mecánico generado por la acreción del $\mathrm{BH}$, está dada por $L_{\mathrm{BH}}=\eta \dot{M}_{\mathrm{BH}} c^{2}$, donde $\eta=0.1$ es la eficiencia estándar de producción de energía que ocurre en las inmediaciones del horizonte de eventos al suponer un disco de acreción geométricamente grueso y ópticamente delgado (Mo et al., 2010; Yuan \& Narayan, 2014), y $c$ es la velocidad de la luz.

A medida que las galaxias satélites orbitan dentro de sus halos huéspedes principales, las componentes de DM y bariónicas en sus propios subhalos están siendo afectadas por numerosos procesos de ambiente. SAG modela la quita de masa producida por fuerzas de marea (TS, por tidal stripping) y/o RPS. El TS actúa sobre cada componente de la galaxia (es decir, gas, estrellas y DM), mientras que el RPS es un proceso hidrodinámico el cual sólo afecta al gas. Así, la remoción de gas en SAG es realizada en dos etapas. Tanto TS como RPS actúan primero sobre el halo de gas caliente: el efecto que sea mayor determinará la cantidad de gas arrancado. Una vez que una fracción considerable del halo caliente es removido, el RPS puede comenzar a afectar al disco de gas frío, siguiendo a Tecce et al. (2010).

Para generar, entonces, la población de galaxias, SAG asigna una galaxia a cada nuevo halo detectado en la simulación de DM, y sigue los árboles de fusión de ese halo para computar la evolución de las propiedades de la galaxia. Cada sistema de halos considerado resulta constituido por sólo una galaxia central del grupo/cúmulo, aquélla asociada al halo huésped principal, y galaxias satélites que pueblan los subalos de DM. Cuando dos halos se fusionan, el más pequeño pierde masa debido a efectos de marea, a medida que orbita la estructura mayor, hasta que el subhalo satélite deja de ser identificado por el buscador de halos. Se supone que la galaxia contenida en este subhalo que desaparece sobrevive hasta que eventualmente se fusiona con la galaxia central del halo huésped. Durante esta etapa temporal, estas galaxias son llamadas galaxias satélites huérfanas. La integración de las órbitas de subhalos que eventualmente albergarán galaxias huérfanas se realiza en una etapa previa a la aplicación del modelo semi-analítico a la simulación de DM. Esta integración tiene en cuenta el pozo de potencial del halo huésped, la pérdida de masa producto del TS y los efectos de fricción dinámica.

Todas estas mejoras se encuentran descritas en detalle en C18 y Cora et al. (2019). A continuación, describimos los aspectos del modelo que se encuentran más relacionados con nuestro trabajo. Debido a que estamos interesadas en estudiar cómo el enriquecimiento químico del gas frío varía dependiendo del modelado de feedback de SN, resumimos las dos variantes del modelado de este proceso en la Sección 2.1.3. A su vez, explicamos el modelo químico implementado en SAG en la Sección 2.1.4. Asimismo, en la Sección 2.1.5 se describe la calibración del modelo. 


\subsubsection{Modelado de feedback de SNs}

El feedback de SN es un proceso crucial que regula la SFR, recalentando el gas frío a partir del cual se forman las estrellas. En versiones anteriores a la utilizada para llevar a cabo la presente investigación, se supuso que la cantidad de masa recalentada producto de las SNs generadas en cada evento de formación estelar es

$$
\Delta M_{\text {reheated }}=\frac{4}{3} \epsilon \frac{\eta E_{\mathrm{SN}}}{V_{\mathrm{vir}}^{2}} \Delta M_{\star},
$$

donde $\eta$ es el número de SNs que surgen a partir de la formación de una población estelar de masa $\Delta M_{\star} . E_{\mathrm{SN}}=10^{51}$ erg es la energía liberada por una $\mathrm{SN}, V_{\text {vir }}$ es la velocidad virial del (sub)halo y $\epsilon$ es la eficiencia del feedback de SN (un parámetro libre del modelo). La cantidad $\Delta M_{\text {reheated }}$ es estimada en cada uno de los pasos de tiempo equidistantes resultantes de la subdivisión de los intervalos entre las salidas de la simulación de DM, que se realiza para integrar las ecuaciones diferenciales que describen los distintos procesos físicos incluidos en SAG.

Este esquema de feedback de SN es modificado en la actual versión de SAG con el propósito de satisfacer restricciones observacionales a alto redshift (C18), mediante la inclusión de nuevos factores que tienen en cuenta una dependencia explícita con el redshift y una modulación adicional con la velocidad virial, como sugieren las simulaciones cosmológicas hidrodinámicas FIRE (Muratov et al., 2015). Entonces, el esquema de feedback produce una masa recalentada de gas dada por

$$
\Delta M_{\text {reheated }}=\frac{4}{3} \epsilon \frac{\eta E_{\mathrm{SN}}}{V_{\mathrm{vir}}^{2}}(1+z)^{\beta}\left(\frac{V_{\mathrm{vir}}}{60 \mathrm{~km} \mathrm{~s}^{-1}}\right)^{\alpha} \Delta M_{\star}
$$

donde el exponente $\alpha$ toma valores -3.2 y -1.0 para velocidades viriales menores y mayores a $60 \mathrm{~km} \mathrm{~s}^{-1}$, respectivamente. La eficiencia, $\epsilon$, y la pendiente de la ley de potencia del redshift, $\beta$, son parámetros libres del modelo.

El gas recalentado es transferido desde la fase fría a la fase caliente, es decir, parte de la masa en el disco de gas frío, que representa al ISM en las galaxias modeladas, es calentado y relocalizado en el halo de gas caliente de la galaxia. Parte de éste retorna luego a la fase fría a través del enfriamiento radiativo, un proceso que tiene lugar tanto en galaxias centrales como en satélites. Encontramos que parte del gas caliente debe ser eyectado hacia afuera del halo, reduciendo la reserva de gas caliente disponible para enfriarse, evitando así un exceso de masa estelar a alto redshift (Guo et al., 2011; Henriques et al., 2013; Hirschmann et al., 2016). Por lo tanto, el nuevo modelo de feedback de SN es complementado con la eyección de masa de gas caliente. La masa eyectada es calculada como

$$
\Delta M_{\text {ejected }}=\frac{\Delta E_{\mathrm{SN}}-0.5 \Delta M_{\text {reheated }} V_{\mathrm{vir}}^{2}}{0.5 V_{\mathrm{vir}}^{2}},
$$

donde $\Delta E_{\mathrm{SN}}$ es la energía inyectada por estrellas masivas (Guo et al., 2011). Esta energía es modelada de una manera similar a la modificada masa recalentada como

$$
\Delta E_{\mathrm{SN}}=\frac{4}{3} \epsilon_{\mathrm{ejec}} \frac{\eta E_{\mathrm{SN}}}{V_{\mathrm{vir}}^{2}}(1+z)^{\beta}\left(\frac{V_{\mathrm{vir}}}{60 \mathrm{~km} \mathrm{~s}^{-1}}\right)^{\alpha} \Delta M_{\star} 0.5 V_{\mathrm{SN}}^{2}
$$

donde $\epsilon_{\text {ejec }}$ es la correspondiente eficiencia, considerada como un parámetro libre del modelo, y $0.5 V_{\mathrm{SN}}^{2}$ es la energía cinética promedio de la eyección de SN por unidad de masa de estrellas formadas, dada por $V_{\mathrm{SN}}=1.9 V_{\text {vir }}^{1.1}$ (Muratov et al., 2015). Con el propósito de reproducir la evolución observada de la función de masa estelar de las galaxias a $z>1$, el (sub)halo necesita reincorporar la masa de gas eyectada previamente dentro de una escala de tiempo que dependa de la inversa de la 
masa del (sub)halo, $M_{\text {vir }}$ (Henriques et al., 2013). De esta manera, la masa reincorporada está dada por

$$
\Delta M_{\text {reinc }}=\gamma \Delta M_{\text {ejected }} \frac{10^{10} \mathrm{M}_{\odot}}{M_{\text {vir }}},
$$

donde $\gamma$ es un parámetro libre que regula la eficiencia del proceso.

\subsubsection{Modelo de enriquecimiento químico}

Las estrellas contribuyen tanto con material reciclado como con nuevos elementos químicos originados por nucleosíntesis que dan lugar al enriquecimiento químico de las fases caliente y fría del gas, y pueden dar como resultado un incremento en la metalicidad de futuras generaciones de estrellas. La masa del elemento químico $j$, que es eyectado por estrellas en un dado rango de masa centrado en $m_{k}$, está dada por

$$
\Delta M_{\mathrm{ej}_{k}}^{j}=\left[R_{k} X^{j}+Y_{k}^{j}\right] \Delta M_{\star}
$$

donde $X^{j}$ es la abundancia solar del elemento $j, R_{k}$ es la fracción reciclada y $Y_{k}^{j}$ es la producción estelar del elemento $j$. Tanto $R_{k}$ como $Y_{k}^{j}$ dependen del rango de masa $k$-ésimo. Este último se refiere al material contribuido por las estrellas luego del proceso de nucleosíntesis, que llamaremos a partir de aquí como yields estelares. La fracción reciclada está dada por

$$
R_{k}=\int_{m_{k}^{i}}^{m_{k}^{s}} \Phi(M) r_{k} \mathrm{~d} M
$$

donde $m_{k}^{i}$ y $m_{k}^{s}$ representan los límites de masa inferior y superior, $\Phi(M)$ es la IMF utilizada (definida en la Ecuación 2.5), y $r_{k}$ es la diferencia entre la masa $m_{k}$ de la estrella al nacer y su masa remanente luego de la pérdida de masa producto de los vientos estelares y/o explosiones de SN II. El yield estelar del nuevo material químico $j$ formado es expresado como

$$
Y_{k}^{j}=\int_{m_{k}^{i}}^{m_{k}^{s}} \Phi(M) p_{k}^{j} \mathrm{~d} M,
$$

donde $p_{k}^{j}$ representa el yield estelar del elemento químico $j$ producido por una estrella con masa en el rango centrado en $m_{k}$.

Debido a que las SNs tipo Ia (SNIa) no dejan remanentes, la correspondiente masa eyectada es estimada usando una expresión diferente, dada por

$$
\Delta M_{\mathrm{ej}(\mathrm{Ia})_{\mathrm{k}}}^{j}=\eta_{\mathrm{Ia}_{k}} m_{\mathrm{Ia}_{k}}^{j} \Delta M_{\star}
$$

donde $m_{\mathrm{Ia}_{k}}^{j}$ es la masa de la especie $j$ eyectada por cada evento de SNIa, y $\eta_{\mathrm{Ia}_{k}}$ es el número de SN Ia por masa estelar formada en un dado rango de masas. Ambas cantidades dependen tanto del modelo adoptado para este tipo de SNs, como del intervalo de masa estelar centrado en $m_{k}$.

El material eyectado por vientos estelares o explosiones de SN contribuye a la masa reciclada, $\Delta M_{\text {recycled }}=\Delta M_{\mathrm{ej}}+\Delta M_{\mathrm{ej}(\mathrm{Ia})}$, la cual es el resultado de la contribución acumulativa de todos los elementos químicos eyectados por estrellas en diferentes rangos de masa (Ecuaciones 2.14 y 2.17) a un dado tiempo, determinada por la combinación de la SFR en cada galaxia y de la escala de tiempo de retorno de todas las fuentes consideradas. Los metales recientemente sintetizados contenidos en $\Delta M_{\text {recycled }}$ contribuyen al aumento de la abundancia química de todas las componentes bariónicas a través de eyecciones y reincorporaciones de gas. 
La energía inyectada resultante de las explosiones de SN recalienta una fracción de gas frío, referida como masa recalentada ( $\left.\Delta M_{\text {reheated }}\right)$, dada por las Ecuaciones 2.9 o 2.10, según el modelo de feedback de SN utilizado. Esta masa recalentada es transferida hacia el halo de gas caliente como un flujo saliente de gas químicamente enriquecido con la metalicidad del gas frío. El nuevo esquema de feedback de SN permite, entonces, la eyección y posterior reincorporación del gas caliente ( $\Delta M_{\text {ejected }}$ y $\Delta M_{\text {reinc }}$ dadas por Ecuaciones 2.11 y 2.13 , respectivamente). Las tasas de enfriamiento, que dependen de la metalicidad, son tenidas en cuenta para calcular la cantidad de gas que se ha enfriado, $\Delta M_{\text {cool }}$, cuya metalicidad está determinada por la abundancia química del gas caliente, y es transportada desde esa fase al disco de gas frío. En este escenario, la variación de masa del elemento químico $j$ contenido en una dada componente bariónica (gas caliente, gas frío, estrellas, o reservorio de gas eyectado) está dada por

$$
\begin{array}{r}
\Delta M_{\text {hot }}^{j}=-\Delta M_{\text {cool }} A_{\text {hot }}^{j}+\Delta M_{\text {reheated }} A_{\text {cold }}^{j} \\
+f_{\text {rec, } \mathrm{h}}\left(\Delta M_{\text {ej }}^{j}+\Delta M_{\text {ej(Ia) }}^{j}\right) \\
-\Delta M_{\text {ejected }} A_{\text {hot }}^{j}+\Delta M_{\text {reinc }} A_{\text {ejected }}^{j}, \\
\Delta M_{\text {cold }}^{j}=\Delta M_{\text {cool }} A_{\text {hot }}^{j}-\Delta M_{\star} A_{\text {cold(SF) }}^{j} \\
+f_{\text {rec,c }}\left(\Delta M_{\text {ej }}^{j}+\Delta M_{\text {ej(Ia) }}^{j}\right)-\Delta M_{\text {reheated }} A_{\text {cold }}^{j}, \\
\Delta M_{\text {stellar }}^{j}=\Delta M_{\star} A_{\text {cold(SF) }}^{j}-\Delta M_{\text {recycled }} A_{\text {star }}^{j}, \\
\Delta M_{\text {ejected }}^{j}=\Delta M_{\text {ejected }} A_{\text {hot }}^{j}-\Delta M_{\text {reinc }} A_{\text {ejected }}^{j},
\end{array}
$$

donde $A_{\mathrm{B}}^{j}=M_{\mathrm{B}}^{j} / M_{\mathrm{B}}$ es la abundancia del elemento químico $j$ en la componente bariónica $\mathrm{B}$, con $M_{\mathrm{B}}^{j}$ la masa del elemento $j$ contenida en la componente B de masa total $M_{\mathrm{B}}$. El subíndice B alude al gas frío, gas caliente, estrellas y gas eyectado. En el último caso, la abundancia es el resultado de la sucesión de eventos de eyección que combinan diferentes metalicidades de gas caliente. La abundancia $A_{\text {cold(SF) }}^{j}$ representa el contenido de masa del elemento $j$ en el gas frío al momento que las estrellas de masa estelar $\Delta M_{\star}$ nacen. $f_{\text {rec, } \mathrm{x}}$ denota la fracción de masa reciclada (combinación de elementos químicos reciclados y recientemente sintetizados) que son agregados a las fases caliente $(\mathrm{x}=\mathrm{h})$ y fría $(\mathrm{x}=\mathrm{c})$.

El destino del material reciclado, que contiene metales que han sido recientemente sintetizados en las estrellas, es determinado por la cantidad relativa de masa recalentada y reciclada. Esta última puede permanecer en el disco de gas frío o ser inyectada al halo de gas caliente. Ambas masas, recalentada y reciclada, son puramente el resultado del proceso de explosión de SN. En la versión de SAG utilizada en nuestro trabajo, se evita el mecanismo de dos pasos en donde el material reciclado es primero completamente diluido en el gas frío, y luego una fracción de éste es transferida a la fase caliente debido a la inyección de energía. Consideramos, en cambio, que si la masa recalentada es menor en comparación con la masa reciclada, entonces una fracción $f_{\text {rec, h }}=\Delta M_{\text {reheated }} / \Delta M_{\text {recycled }}$ del material reciclado es agregada al halo de gas caliente, y el resto $\left(f_{\text {rec, } \mathrm{c}}=1-f_{\text {rec,c }}\right)$ permanece en el disco de gas frío (ver el tercer término de las Ecuaciones 2.18 y 2.19). Por el contrario, si la masa recalentada es más grande que la masa reciclada, esta última es directamente agregada al halo de gas caliente $\left(f_{\text {rec, } \mathrm{h}}=1\right.$ y $\left.f_{\text {rec, c }}=0\right)$, significando que ésta ha sido completamente recalentada. A este esquema de recilado lo llamaremos, de aquí en más, esquema de reciclado $\mathcal{A}$.

También evaluamos una variante del modelo SAG que considera un criterio más simple para decidir el destino del material reciclado. En este nuevo esquema (al que llamaremos esquema de reciclado 
$\mathcal{B})$, la fracción de material reciclado que es agregada al gas frío es proporcional a la fracción de gas frío de toda la galaxia, es decir, el cociente entre el contenido de gas y la suma de gas frío y estrellas. Por lo tanto, la fracción involucrada en el tercer término de las Ecuaciones 2.18 y 2.19 es reemplazada por $f_{\text {rec, } \mathrm{c}}=M_{\text {cold }} /\left(M_{\text {cold }}+M_{\star}\right)$ y $f_{\text {rec, h }}=1-f_{\text {rec, } \mathrm{c}}$, respectivamente. En cualquiera de estos dos esquemas, se da por sentado que los elementos químicos contenidos en la masa recalentada son mezclados instantáneamente con los metales que ya se encuentran en la correspondiente fase. Entonces, el gas frío aumenta su abundancia de metales a través de dos canales, específicamente, la contribución directa de material reciclado y la caída de gas frío enriquecido, cuya metalicidad es determinada por la metalicidad del gas caliente. En un determinado momento, todas las componentes bariónicas tienen una distribución uniforme de masa y metales, dado que SAG no sigue las dependencias radiales de estas cantidades. Recordemos que esto es una característica común de los SAMs.

SAG sigue la abundancia de once elementos químicos diferentes $\left(\mathrm{H},{ }^{4} \mathrm{He},{ }^{12} \mathrm{C},{ }^{14} \mathrm{~N},{ }^{16} \mathrm{O},{ }^{20} \mathrm{Ne}\right.$, ${ }^{24} \mathrm{Mg},{ }^{28} \mathrm{Si},{ }^{32} \mathrm{~S},{ }^{16} \mathrm{Ca},{ }^{56} \mathrm{Fe}$ ). Considera los yields estelares dependientes de la masa estelar, $p_{k}$, especificados en Gargiulo et al. (2015), que fueron estimados para la IMF de Chabrier (Chabrier, 2003). Se adopta el total de masa eyectada de los modelos de metalicidad solar, siguiendo las abundancias solares de Anders \& Grevesse (1989), con una composición solar de $\mathrm{Z}_{\odot}=0.02$. La dependencia con la metalicidad estelar de los yields estelares no está incluida en el modelo debido a que produce un efecto mínimo en las propiedades de las galaxias estudiadas aquí, a su vez que incrementa el costo computacional del modelo significativamente. Las escalas de tiempo de retorno del material eyectado por todas las fuentes consideradas son obtenidas a partir de los tiempos de vida de estrellas dado por Padovani \& Matteucci (1993). Las tasas de enfriamiento de gas son estimadas considerando la potencia radiativa total por elemento químico dada por Foster et al. (2012).

\subsubsection{Calibración del modelo SAG}

La calibración del modelo SAG consiste en la estimación de sus parámetros libres. La misma se realiza mediante la implementación de la técnica de Optimización de Enjambre de Partículas (o Particle Swarm Optimization, PSO) presentada en Ruiz et al. (2015). Esta herramienta numérica permite afinar el valor de los parámetros libres a través de la exploración del espacio de parámetros de SAG por medio de caminatas aleatorias de un dado conjunto de "partículas" (equivalente a las cadenas de Markov Monte Carlo) que comparten información entre ellas. Al comparar los resultados del modelo con un dado conjunto de observables (es decir, imponiendo como restricciones propiedades observadas de galaxias), el método PSO construye un conjunto de los valores más adecuados para los parámetros libres.

\begin{tabular}{lr}
\hline Parámetro & SAG \\
\hline$\alpha$ & 0.0402 \\
$\epsilon$ & 0.3299 \\
$\epsilon_{\text {ejec }}$ & 0.02238 \\
$\gamma$ & 0.0555 \\
$\beta$ & 1.99 \\
$f_{\text {BH }}$ & 0.0605 \\
$\kappa_{\text {AGN }}$ & $3.02 \times 10^{-5}$ \\
$f_{\text {pert }}$ & 14.557 \\
$f_{\text {hot,sat }}$ & 0.2774 \\
\hline
\end{tabular}

Tabla 2.1: Valores con mejor ajuste de los parámetros libres obtenidos a través de la técnica PSO para el modelo SAG. Estos valores definen la variante del modelo que referimos como $\mathrm{SAG}_{\beta 1.9}$. 


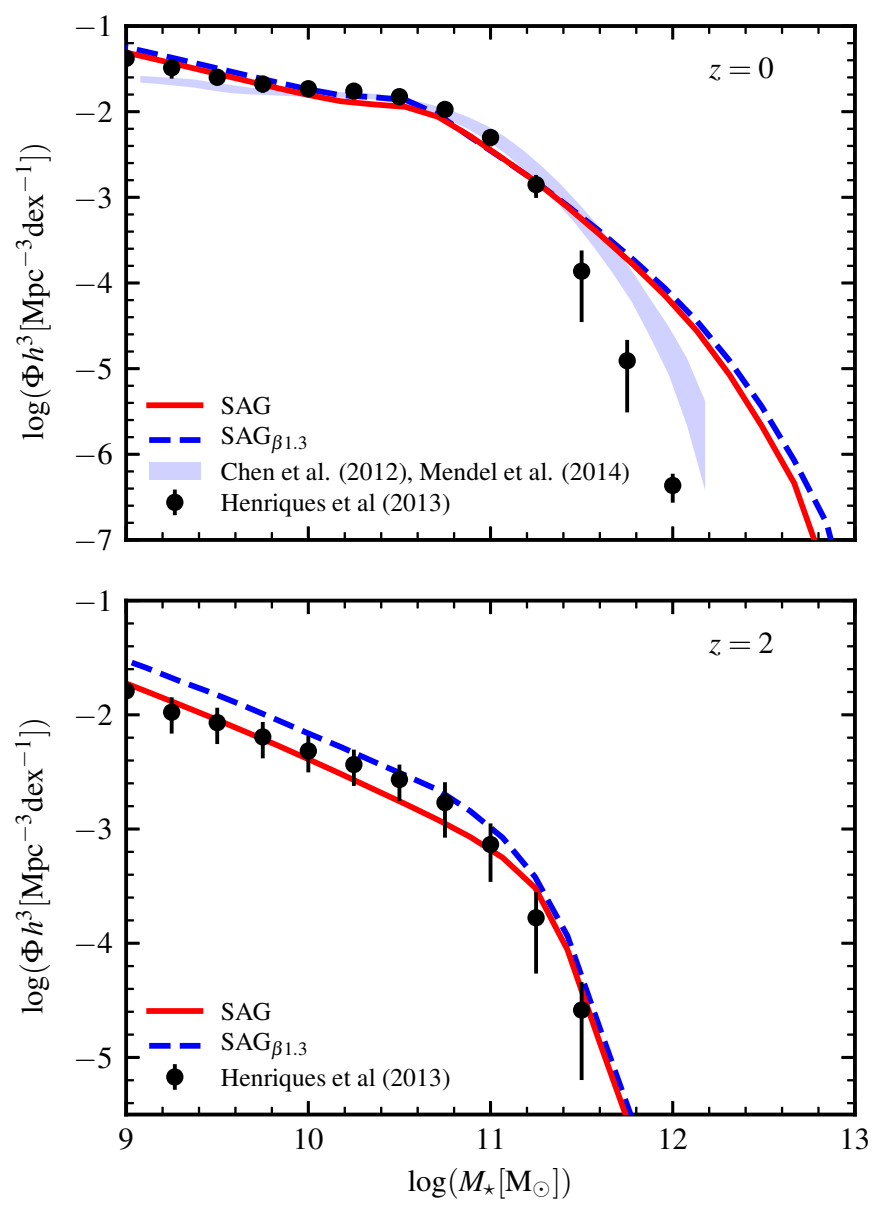

Figura 2.1: Función de masa estelar usada como restricción para calibrar el modelo SAG. La línea roja sólida representa el modelo $\mathrm{SAG}_{\beta 1.9}$ mientras que la línea azul a trazos representa el modelo $\mathrm{SAG}_{\beta 1.3}$, ambos a redshift $z=0$ (panel superior) y $z=2$ (panel inferior). Los datos observacionales de Henriques et al. (2013) son representados con símbolos negros, junto a sus barras de errores. El acuerdo entre modelo y observación es bastante bueno, excepto por el exceso a altas masas a $z=0$. Este exceso resulta menos evidente cuando se compara con las estimaciones compiladas y analizadas por Bernardi et al. (2017, área gris), la cual incluye datos de Chen et al. (2012) y Mendel et al. (2014). Figura adoptada de C18.

Para la actual versión de SAG, nueve parámetros libres son considerados para el proceso de calibración. Los parámetros libres son la eficiencia de formación estelar ( $\alpha$, Ecuación 2.3), la eficiencia de feedback de SN de las estrellas formadas tanto en el disco como en el bulbo ( $\epsilon$, Ecuación 2.10), la eficiencia de eyección del gas desde la fase caliente $\left(\epsilon_{\text {ejec }}\right.$, Ecuación 2.12) y de reincorporación $(\gamma$, Ecuación 2.13), el exponente que regula la evolución con el redshift de la masa recalentada y eyectada ( $\beta$, Ecuación 2.10), el crecimiento de los BHs masivos ( $f_{\mathrm{BH}}$, Ecuación 2.6) y la eficiencia de feedback de AGN ( $\kappa_{\mathrm{AGN}}$, Ecuación 2.7), el factor involucrado en la perturbación de la escala de distancia que genera los eventos de inestabilidad de discos ( $f_{\text {pert }}$, Ruiz et al. 2015, C18), y el factor que determina el destino del gas frío recalentado ( $f_{\text {hot,sat }}$, Ruiz et al. 2015, C18). Los valores de estos parámetros que mejor ajustan al modelo pueden verse en la Tabla 2.1. El conjunto de observables usados para el proceso de calibración conlleva cinco restricciones observacionales que se detallan a continuación. 


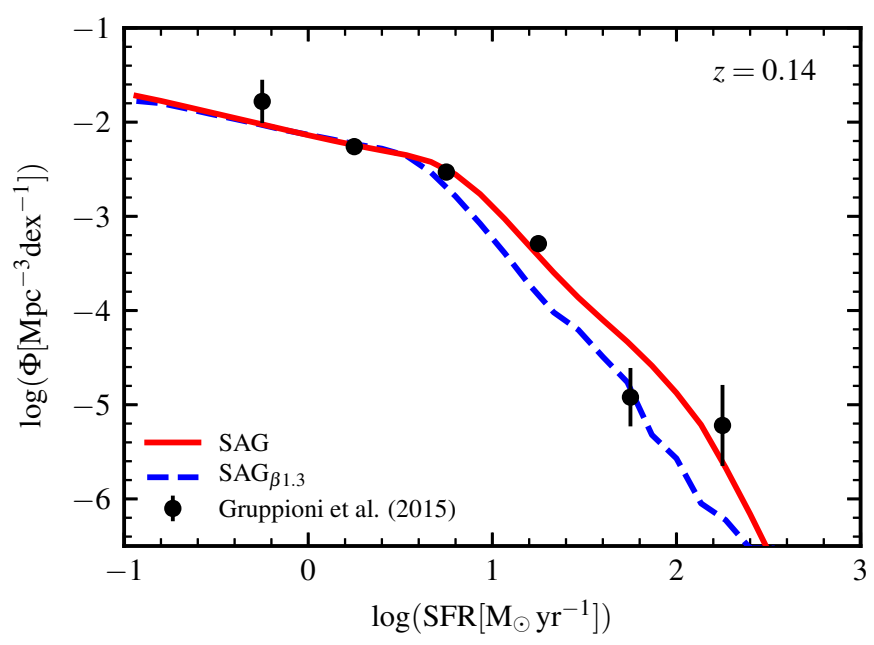

Figura 2.2: Función de distribución de la tasa de formación estelar a $z=0.14$. La línea roja sólida representa los resultados del modelo $\mathrm{SAG}_{\beta 1.9}$, mientras que la línea azul a trazos muestra los resultados del modelo $\mathrm{SAG}_{\beta 1.3}$. Los círculos negros simbolizan las observaciones de Gruppioni et al. (2015). Figura adoptada de C18.

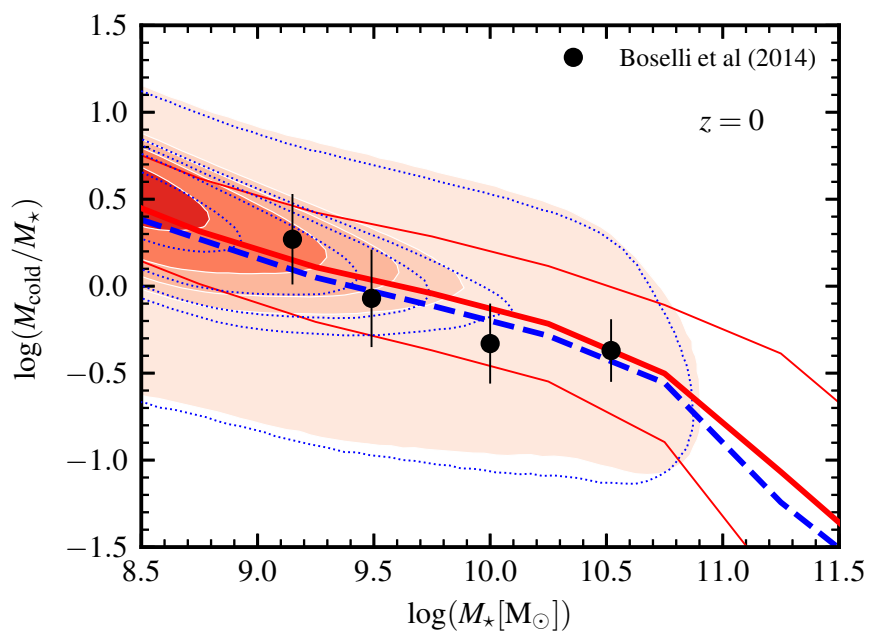

Figura 2.3: Fracción de masa de gas frío como función de la masa estelar a $z=0$, usado como restricción para calibrar el modelo SAG. Las líneas rojas gruesa y fina representan los valores promedio de la relación para las galaxias del modelo $\mathrm{SAG}_{\beta 1.9}$ y su desviación estándar, respectivamente. La línea azul a trazos muestra la mediana de la relación para el modelo $\mathrm{SAG}_{\beta 1.3}$, mientras que los contornos azules denotan la desviación estándar. Los contornos representan el máximo número de densidad de puntos por cada muestra, con niveles $[0.01,0.19,0.26,0.38,0.69,0.95,0.997]$. Esta relación es comparada con las observaciones de Boselli et al. (2014) representadas mediante círculos negros con sus barras de error. Figura adoptada de C18.

Para calibrar la actual versión del modelo SAG se utilizaron cinco restricciones observacionales. Por un lado, se utiliza la función de masa estelar (SMF, por stellar mass function) a $z=0$ y a $z=2$ (Figura 2.1). La nueva estimación de la masa recalentada es lo que permite que haya un buen acuerdo con este observable no sólo a bajo redshift sino también a alto redshift. Por otro lado, se utiliza la función distribución de la tasa de formación estelar (SFRF, por star formation rate function) a $z=0.14$ (Figura 2.2). Esta función representa la densidad numérica de galaxias en cierto intervalo de SFR, y ayuda a recuperar la evolución observada de la SFR y el crecimiento de la masa de las galaxias. 
El hecho que el crecimiento de las galaxias y el cese de la formación estelar son comportamientos bien plasmados por SAG resulta evidente en el contenido de gas frío de las galaxias modeladas. Así, la fracción de masa de gas frío como función de la masa estelar (CGMF, por cold gas mass fraction) obtenida por SAG (Figura 2.3), la cual tiene un comportamiento esperado dentro de los rangos de masa estelar considerados para la calibración del modelo $\left(\log \left(M_{\star} / \mathrm{M}_{\odot}\right) \in[9.15,10.52]\right)$, resulta el cuarto observable utilizado para la calibración. Por último, se utiliza la relación entre la masa de los BHs supermasivos centrales y la masa del bulbo (Figura 2.4). En todos los casos, se compara al modelo $\mathrm{SAG}$ de referencia, $\mathrm{SAG}_{\beta 1.9}$, con un modelo que varía en el valor del parámetro que regula la eficiencia con el redshift del calentamiento y eyección de masa por feedback de $\mathrm{SN}, \mathrm{SAG}_{\beta 1.3}$ (detallado en la Sección 2.1.6).

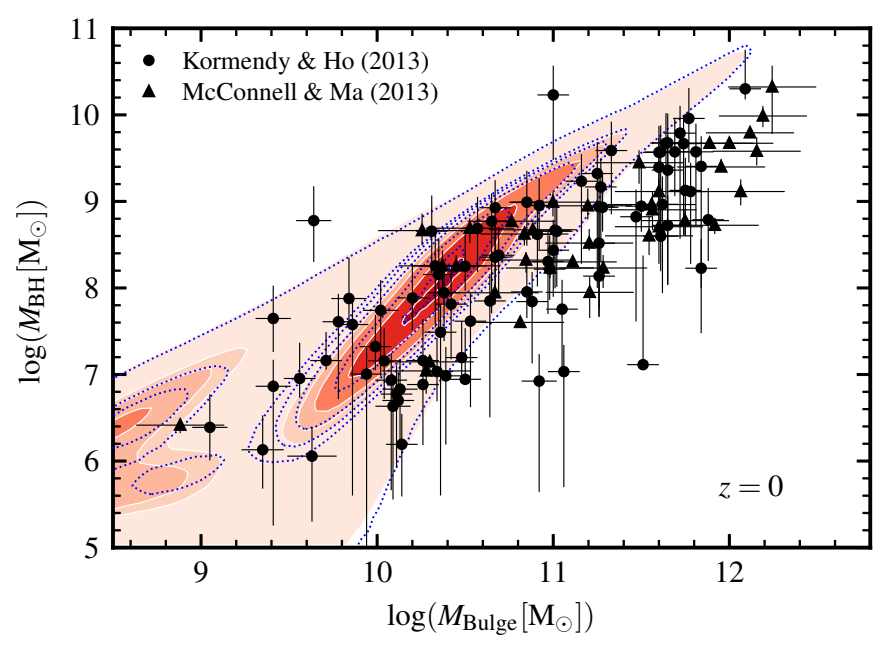

Figura 2.4: Relación entre el agujero negro central y la masa del bulbo de la galaxia a $z=0$, usado como restricción para calibrar el modelo SAG. Los niveles de contorno son los mismos que aquéllos de la Figura 2.3 (rojo para el modelo $\mathrm{SAG}_{\beta 1.9}$ y azul para el modelo $\mathrm{SAG}_{\beta 1.3}$ ). Los datos observacionales fueron obtenidos de Kormendy \& Ho (2013) y McConnell \& Ma (2013), representados por círculos y triángulos negros, respectivamente. Figura adoptada de C18.

\subsubsection{Definiciones de variantes del modelo}

La calibración de SAG da como resultado un valor del exponente que regula la dependencia de la eficiencia de feedback de SN con el redshift, $\beta=1.99$, el cual es más alto que el sugerido por Muratov et al. (2015, $\beta=1.3$ ). Como se discute en $\mathrm{C} 18$, este valor es una consecuencia de las restricciones impuestas para el rango de menor masa estelar de la SMF a $z=2$, lo que genera una subestimación de la densidad de SFR cósmica (SFRD, por star formation rate density) a alto redshift (ver Sección 3.3.2). Las predicciones del modelo, como la SFRD y la fracción de galaxias pasivas como función de la masa estelar, masa de halo y distancia al centro del halo, se encuentran en mejor acuerdo con datos observacionales cuando se adopta el valor $\beta=1.3$, a su vez que se mantienen los valores del mejor acuerdo encontrados en la calibración para el resto de parámetros libres. Esta variante del modelo se denominará de aquí en más como $\mathrm{SAG}_{\beta 1.3}$ (al igual que se hizo en C18).

El análisis del modelo SAG calibrado y el modelo $\mathrm{SAG}_{\beta 1.3}$ permite evaluar el impacto de distintas versiones de feedback de SN a alto redshift en el desarrollo de la MZR. De aquí en más, nos referiremos al modelo fiduciario como $\mathrm{SAG}_{\beta 1.9}$. También analizamos la influencia de otros cambios introducidos en el modelado de feedback de SN al considerar una versión de SAG en donde el actual modelo de feedback (Ecuación 2.10) es reemplazado por la receta original adoptada para este proceso (Ecuación 2.9), anulando el proceso de eyección y reincorporación, y manteniendo los mismos valo- 
res de calibración de los parámetros libres presentados en la Tabla 2.1 (a excepción de $\beta$ que tendrá el valor de 0). Nos referiremos a esta corrida como $\mathrm{SAG}_{\beta 0.0}$. Notar que esta etiqueta no sólo significa que la masa recalentada pierde la dependencia explícita con el redshift, sino que además indica la falta de modulación adicional con la velocidad virial de la Ecuación 2.10. Para evaluar el impacto del esquema de reciclado de masa adoptado por el modelo fiduciario, y así decidir el destino de la masa reciclada en la evolución de la MZR, definimos una nueva variante del modelo en donde el correspondiente esquema de $\mathrm{SAG}_{\beta 1.9}$ (esquema $\mathcal{A}$ ) es reemplazado por una receta más simple (esquema $\mathcal{B}$ ) que depende de la fracción de gas frío; este modelo es llamado $\mathrm{SAG}_{\beta 1.9-\mathrm{Rec}}$. El análisis de los resultados de estas cuatro variantes del modelo nos ayuda a desentrañar los procesos físicos encargados de la ausencia o presencia de evolución de la MZR.

\subsection{Simulaciones hidrodinámicas cosmológicas EAGLE}

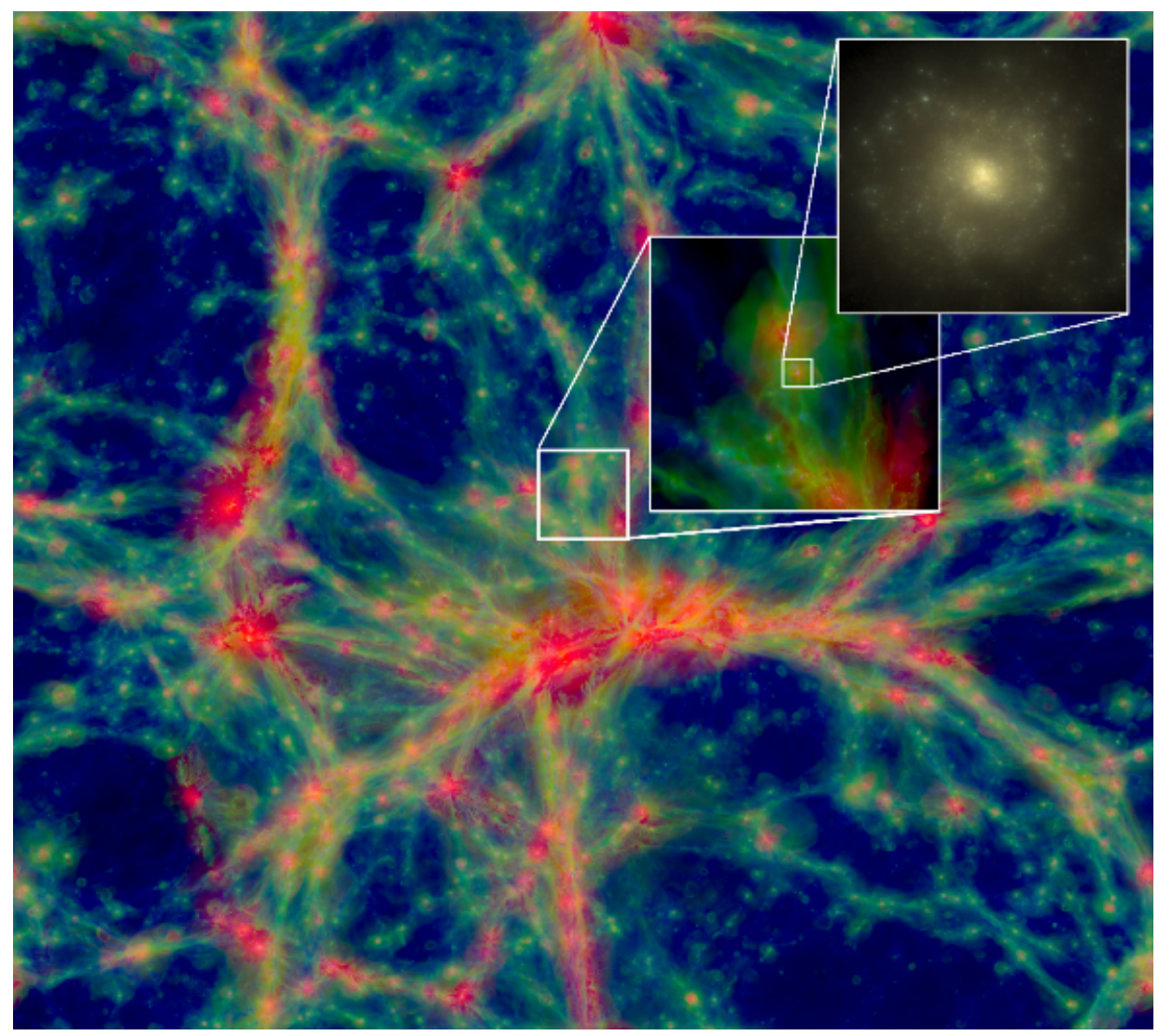

Figura 2.5: Corte de $100 \times 100 \times 20 \mathrm{cMpc}$ de la simulación Ref-L100N1504 a $z=0$. La intensidad muestra la densidad del gas, mientras que los colores representan temperaturas del gas. Temperaturas $<10^{4.5} \mathrm{~K}$ se encuentran en azul, en verde se muestran temperaturas de $10^{4.5} \mathrm{~K}<T<10^{5.5} \mathrm{~K}$, y en rojo las temperaturas $>10^{5.5} \mathrm{~K}$. El recuadro muestra regiones de $10 \mathrm{cMpc}$ y $60 \mathrm{ckpc}$ de lado y se acerca para ver en detalle una galaxia individual con $M_{\star}=3 \times 10^{10} \mathrm{M}_{\odot}$. La imagen de $60 \mathrm{ckpc}$ muestra la luz estelar basada en los filtros monocromáticos $u, g$ y $r$ del SDSS, y teniendo en cuenta la distribución de polvo. Esta imagen fue creada usando el código de transferencia radiativa SKIRT (Baes et al., 2011; Trayford et al., 2017). Figura adoptada de S15.

El proyecto EAGLE es un conjunto de simulaciones cosmológicas hidrodinámicas de diferentes tamaños de caja, número de partículas, resoluciones numéricas y modelados de física de subgrilla. Estas simulaciones están descritas en S15 y Crain et al. (2015), mientras que los datos públicos disponi- 
bles se encuentran documentados en McAlpine et al. (2016). EAGLE sigue la evolución de partículas de DM y gas, en una cosmología plana $\Lambda$ CDM (Planck Collaboration et al., 2014b) regida por los parámetros: densidad de materia $\Omega_{\mathrm{m}}=0.307$, densidad de energía $\Omega_{\Lambda}=0.693$, densidad bariónica $\Omega_{\mathrm{B}}=0.04825$, índice espectral $n_{\mathrm{s}}=0.9611$, constante de Hubble $H_{0}=100 \mathrm{~h}^{-1} \mathrm{~km} \mathrm{~s}^{-1} \mathrm{Mpc}^{-1}$, con $h=0.6777$, y abundancia primordial del helio $Y=0.248$.

Un algoritmo Friend-of-Friends (FOF, Davis et al. 1985) es implementado para identificar los halos de partículas de DM; mientras que el algoritmo SUBFIND (Springel et al., 2001; Dolag et al., 2009) es el encargado de identificar los subhalos unidos gravitacionalmente dentro de la estructura FOF, uniendo las partículas de gas y estrellas con las partículas de DM más cercanas. Estos sughalos son, entonces, donde se albergan las galaxias de la simulación. Por cada halo FOF, el subhalo que contenga la partícula con el menor valor de pozo de potencial es definida como la galaxia central, mientras que el resto de los subhalos se catalogan como galaxias satélites.

Una de las ventajas de EAGLE es que varias de sus simulaciones cuentan con un gran rango dinámico, el cual puede verse en la Figura 2.5. Esto permite estudiar la cosmología del Universo al mismo tiempo que se pueden estudiar a las galaxias de manera resuelta con gran detalle. Por otro lado, una característica importante que hace que las simulaciones EAGLE tengan gran relevancia en el estudio de la formación y evolución de galaxias es que implementa un feedback térmico en lugar de uno cinético (ver Sección 2.2.2). Además, el feedback energético varía con las propiedades locales del gas y las fuerzas termodinámicas nunca se apagan. Esto está en contraste con otras simulaciones hidrodinámicas, como Illustris (Vogelsberger et al., 2014) o las simulaciones de Puchwein \& Springel (2013), las cuales implementan un feedback cinético, escalan la velocidad de los vientos con la dispersión de velocidades de la DM, determinan la dependencia de carga de masa de los vientos con la dispersión de velocidades de DM al suponer una energía de vientos constante, y temporalmente apagan las fuerzas hidrodinámicas de las partículas de los vientos permitiendo que escapen de las galaxias. Las diferencias implementadas en el modelado de la física de las simulaciones EAGLE permite que se puedan reproducir de mejor manera diversos observables, tanto durante el proceso de calibración como en sus predicciones, como por ejemplo sucede con la función de masa estelar de galaxias (GSMF, por galaxy stellar mass function).

\subsubsection{Técnica SPH utilizada}

Para correr las simulaciones EAGLE, se realizó una modificación al código GADGET-3 (Springel, 2005), el cual aplica la técnica SPH de $N$-cuerpos Tree-PM (por Particle Mesh). Los métodos numéricos utilizados para esta modificación se definieron como ANARCHY (Schaller et al., 2015). Las simulaciones SPH usan un conjunto de partículas rastreadoras para describir el estado de un fluido, con las cantidades del fluido continuo definidas por una técnica de interpolación de núcleo (o kernel) (Gingold \& Monaghan, 1977; Lucy, 1977; Monaghan, 1992). Las partículas con coordenadas $\overrightarrow{r_{i}}$, velocidades $\overrightarrow{v_{i}}$ y masas $m_{i}$ son pensados como elementos de fluido que muestrean el gas en un sentido Lagrangiano. El estado termodinámico de cada elemento de fluido puede ser definido en términos de su energía térmica por unidad de masa, $u_{i}$, o en términos de la entropía por unidad de masa, $s_{i}$. Aquí se prefiere usar el último dado que la variable termodinámica independiente evoluciona en la SPH (discutido en detalle en Springel \& Hernquist 2002). Este tipo de formulación SPH permite, entonces, conservar tanto la energía como la entropía incluso cuando se usa un suavizado adaptativo completo. Este método resulta en oposición a la formulación SPH tradicional, la cual puede violar la conservación de entropía en ciertas situaciones.

Los métodos Lagrangianos como los SPH son particularmente adecuados para seguir el crecimiento gravitacional de las estructuras y para incrementar automáticamente la resolución en las regiones centrales de los halos galácticos, las cuales son las regiones de principal interés para la formación y evolución de galaxias (Springel, 2005). El tratamiento adecuado de la auto-gravitación del gas en una manera consistente con la DM es una gran ventaja de los métodos SPH basados en partículas. Otra 
diferencia fundamental con los esquemas basados en mallas (o métodos Eulerianos, Sección 1.1.2) es que las cantidades termodinámicas transferidas en el flujo no se mezclan entre los diferentes elementos de fluido, a menos que explícitamente se lo modele. Una desventaja importante de los SPH es que el método debe depender de una viscosidad artificial para suministrar la inyección de entropía necesaria en los choques. Los choques son expandidos sobre la escala suavizada de SPH, en lugar de ser resuelta como verdaderas discontinuidades.

\subsubsection{Modelos físicos de subgrilla implementados en EAGLE}

Debido a que las simulaciones hidrodinámicas carecen de la resolución suficiente para modelar procesos que suceden a escala sub-kpc, resulta necesario implementar modelos de subgrilla (los cuales mencionamos en el Capítulo 1). Los modelos de subgrilla son representaciones de importantes procesos físicos que se desarrollan a pequeña escala (como la formación estelar o el feedback), que las simulaciones hidrodinámicas por sí solas no llegan a resolver. Estos modelos de subgrilla son similares a los implementados en los SAMs. En esta Sección, describimos los modelos físicos de subgrilla implementados en las simulaciones EAGLE. Los parámetros libres que regulan estos modelos están detallados en la Sección 2.3.

\section{Enfriamiento Radiativo}

El enfriamiento radiativo y la foto-ionización son implementados elemento a elemento siguiendo a Wiersma et al. (2009a), incluyendo los 11 elementos químicos que resultan importantes: hidrógeno $\mathrm{H}$, helio $\mathrm{He}$, carbono $\mathrm{C}$, nitrógeno $\mathrm{N}$, oxígeno $\mathrm{O}$, neón $\mathrm{Ne}$, magnesio $\mathrm{Mg}$, silicio $\mathrm{Si}$, azufre $\mathrm{S}$, y hierro Fe. Wiersma et al. (2009a) usan la versión 07.02 de CLOUDY (Ferland et al., 1998) para tabular las tasas de enfriamiento como función de la densidad, la temperatura y el redshift, suponiendo que el gas se encuentra en equilibrio de ionización y es expuesto al CMB. A su vez, utiliza el modelo de Haardt \& Madau (2001) para describir la evolución de los rayos ultravioletas y X de fondo provenientes de galaxias y quasares. Al computar las tasas para cada elemento, se tiene en cuenta no sólo las variaciones en metalicidad, sino también las variaciones por abundancias relativas de los elementos. Hay que tener en cuenta que las tasas de enfriamiento podrían estar sobreestimadas en ciertas situaciones debido a la suposición de equilibrio de ionización y por la falta de fuentes locales de radiación ionizante (ejemplo, enfriamiento rápido del gas o gas recientemente expuesto a radiación proveniente de un AGN local).

Se ha elegido ignorar la autoprotección (o self-shielding), la cual puede causar una subestimación de las tasas de enfriamiento de gas denso. Esto se debe a que el self-shielding jugaría un rol importante a densidades $n_{\mathrm{H}}>10^{-2} \mathrm{~cm}^{-3}$ y $T \lesssim 10^{4} \mathrm{~K}$ (Rahmati et al., 2013b). Sin embargo, en tales condiciones se espera que la radiación de fondo sea más importante (Schaye, 2001).

\section{Reionización}

La reionización del hidrógeno es implementada al prender la ionización de fondo espacialmente uniforme y dependiente del tiempo de Haardt \& Madau (2001). Esto se realiza a $z=11.5$, consistente con las mediciones profundas en el óptico de la Planck Collaboration et al. (2014a). A alto redshift se usa una red de tasa de enfriamiento para el gas expuesto al CMB y a la fotodisociación de fondo obtenida al cortar el espectro de Haardt \& Madau (2001) a $z=9$.

Para tener en cuenta el aumento de las tasas de foto-ionización durante la reionización relativa a las tasas ópticamente delgadas supuestas, se inyecta $2 \mathrm{eV}$ por masa de protón. Esto asegura que el gas fotoionizado sea rápidamente calentado a una temperatura de $\sim 10^{4} \mathrm{~K}$. Para el $\mathrm{H}$ esto se realiza instantáneamente, pero para el He II un calor extra es distribuído como función del redshift con una gaussiana centrada en $z=3.5$ y ancho $\sigma(z)=0.5$. Wiersma et al. (2009b) mostraron que esta elección resulta en buen acuerdo con la historia térmica del gas intergaláctico medido por Schaye et al. (2000). 


\section{Formación Estelar}

La formación estelar es implementada siguiendo a Schaye \& Dalla Vecchia (2008), pero con el límite en la densidad dependiente de la metalicidad de Schaye (2004) y un límite en temperatura diferente, como se describe a continuación. Contrariamente a la práctica estándar, se toma la tasa de formación estelar dependiente de la presión en lugar de la densidad. Como se demostró en Schaye \& Dalla Vecchia (2008), esto tiene dos ventajas importantes. Primero, bajo la suposición de que el gas es auto-gravitante, se puede reescribir la ley observacional de formación estelar Kennicutt-Schmidt (KS, Kennicutt 1998), $\dot{\Sigma}_{\star}=A\left(\Sigma_{\mathrm{g}} / 1 M_{\odot} \mathrm{pc}^{-2}\right)^{n}$, como una ley dependiente de la presión

$$
\dot{m}_{\star}=m_{\mathrm{g}} A\left(1 \mathrm{M}_{\odot} \mathrm{pc}^{-2}\right)^{-n}\left(\frac{\gamma}{G} f_{\mathrm{g}} P\right)^{(n-1) / 2},
$$

donde $m_{\mathrm{g}}$ es la masa de la partícula de gas, $\gamma=5 / 3$ es el cociente del calor específico, $G$ es la constante de gravitación, $f_{\mathrm{g}}$ es la fracción de masa de gas de la partícula de gas (se supone que es igual a la unidad), y $P$ es la presión total. Entonces, los parámetros libres $A$ y $n$ son determinados por observaciones del gas y la densidad superficial de la tasa de formación estelar, por lo que no se necesita ninguna afinación. En segundo lugar, si se impone la ecuación de estado, $P=P_{\text {ede }}(\rho)$, luego la ley observacional de formación estelar KS será reproducida sin cambiar los parámetros de la formación estelar. En contraste, si la formación estelar es implementada usando la densidad de volumen en lugar de la presión, la predicción de la ley KS dependerá del grueso del disco y de la ecuación de estado del gas SF. Entonces, en este caso la ley de formación estelar no sólo tiene que ser calibrada, sino que tiene que ser re-calibrada si la ecuación de estado impuesta se cambia. En la práctica, esto rara vez se realiza.

La Ecuación 2.22 se implementa estocásticamente. La probabilidad de que una partícula de gas sea convertida a una partícula estelar sin colisiones durante un paso de tiempo $\Delta t$ es el $\min \left(\dot{m}_{\star} \Delta t / m_{\mathrm{g}}\right.$, 1). Se utiliza $A=1.515 \times 10^{-4} \mathrm{M}_{\odot} \mathrm{yr}^{-1} \mathrm{kpc}^{-2}$ y $n=1.4$, donde se decrece la amplitud por un factor 1.65 relativo al valor usado por Kennicutt (1998) debido a que las simulaciones EAGLE usan una IMF de Chabrier en lugar de una de Salpeter. Se incrementa $n$ al valor 2 para $n_{\mathrm{H}}>10^{3} \mathrm{~cm}^{-3}$, dado que hay evidencia de que es necesario para estrellas que se forman en tan altas densidades, aunque sólo el $\sim 1 \%$ resulta afectado.

Se observa que la formación estelar ocurre en gas molecular frío ( $T<<10^{4} \mathrm{~K}$; Leroy et al. 2008). Debido a que las simulaciones cosmológicas de gran volumen, como las que usamos aquí, carecen de la resolución y el modelo físico de la fase de gas interestelar frío, es apropiado imponer un límite a la formación estelar en la densidad en la que la fase fría se espera que se forme. En las simulaciones hidrodinámicas cosmológicas OWLS (OverWhelmingly Large Simulations; Schaye et al. 2010) se usó un límite constante de $n_{\mathrm{H}}^{*}=10^{-1} \mathrm{~cm}^{-3}$, cuya motivación surge de las consideraciones teóricas y lleva a una densidad superficial de gas crítica de $\sim 10 \mathrm{M}_{\odot} \mathrm{pc}^{-2}$ (Schaye, 2004; Schaye \& Dalla Vecchia, 2008). La densidad de volumen crítica, $n_{\mathrm{H}}=10^{-1} \mathrm{~cm}^{-3}$, es también similar al valor usado en otros trabajos de comparable resolución (Springel \& Hernquist, 2003; Vogelsberger et al., 2013). Aquí en cambio usamos el límite de densidad dependiente de la metalicidad de Schaye (2004),

$$
n_{\mathrm{H}}^{*}=10^{-1} \mathrm{~cm}^{-3}\left(\frac{Z}{0.002}\right)^{-0.64},
$$

donde $Z$ es la metalicidad gaseosa (es decir, la fracción de masa de gas en elementos más pesados que el helio). En el código, el límite es evaluado como una densidad de masa más que la densidad numérica total del hidrógeno. Para prevenir dependencias adicionales con la fracción de masa del hidrógeno (más allá de las implicadas por la Ecuación 2.23), convertimos $n_{\mathrm{H}}$ a una densidad de masa suponiendo una fracción de masa inicial del hidrógeno, $X=0.752$. Debido a que la relación de Schaye (2004) diverge a bajas metalicidades, se impone el límite superior de $n_{\mathrm{H}}^{*}=10 \mathrm{~cm}^{-3}$. Para prevenir la formación estelar en bajas sobredensidades de gas a alto redshift, se requiere que la densidad del gas 
exceda 57.7 veces la densidad cósmica media, aunque los resultados resultan no ser sensibles a este valor (S15).

La dependencia con la metalicidad surge por el hecho de que la transición del gas tibio neutro a gas frío molecular ocurre a densidades y presiones bajas si la metalicidad, y entonces también el cociente polvo-a-gas, es mayor. La fase de transición se desplaza a presiones menores si la metalicidad incrementa debido a una mayor tasa de formación de hidrógeno molecular, al incremento del gas enfriado debido a metales, y al incremento de efectos por polvo (ejemplo, Schaye, 2001). El gas que se encuentra por debajo del punto donde la ley KS es más empinada decrece al aumentar la metalicidad debido a este límite de densidad dependiente de la metalicidad.

Dado que las simulaciones EAGLE no modelan la fase de gas frío, se impone una temperatura piso, $T_{\text {ede }}\left(\rho_{\mathrm{g}}\right)$, correspondiente a la ecuación de estado $P_{\text {ede }} \propto \rho_{\mathrm{g}}^{4 / 3}$, normalizada a $T_{\text {ede }}=8 \times 10^{3} \mathrm{~K}$ a $n_{\mathrm{H}}=10^{-1} \mathrm{~cm}^{-3}$, una temperatura que es típica para el ISM templado. La pendiente $4 / 3$ garantiza que la masa de Jeans y el radio de la longitud de Jeans en el kernel SPH sean independientes de la densidad, lo cual previene la fragmentación espúrea debido a resoluciones finitas (Schaye \& Dalla Vecchia, 2008). Siguiendo a Dalla Vecchia \& Schaye (2012), el gas es apto para formar estrellas si $\log _{10} T<\log _{10} T_{\text {ede }}+0.5$ y $n_{\mathrm{H}}>n_{\mathrm{H}}^{*}$.

Además de la presión mínima correspondiente a la ecuación de estado con pendiente $4 / 3$, se impone una temperatura piso de $8000 \mathrm{~K}$ para densidades $n_{\mathrm{H}}>10^{-5} \mathrm{~cm}^{-3}$ para prevenir que partículas muy enriquecidas se enfríen a temperaturas características del gas frío interestelar. Esto se hace debido a que, como no se modelan los procesos físicos, no se desea incluir la fase fría interestelar. Sólo se impone el límite para densidades $n_{\mathrm{H}}>10^{-5} \mathrm{~cm}^{-3}$, dado que no se debe evitar la existencia de gas frío, adiabático e intergaláctico, el cual está modelado adecuadamente.

\section{Pérdida de masa estelar y SNs Tipo Ia}

Las partículas estelares son tratadas como poblaciones estelares simples (SSPs, por single stellar populations) con una IMF de Chabrier en el rango de $0.1-100 \mathrm{M}_{\odot}$. La implementación de la pérdida de masa estelar está basada de Wiersma et al. (2009b). A cada paso de tiempo y para cada partícula estelar, se computa el valor de la masa estelar que alcanza el fin de la secuencia principal usando tiempos de vida dependientes de la metalicidad de Portinari et al. (1998). Se utiliza la fracción de masa inicial de las partículas que alcanzan esta etapa, la abundancia inicial de elementos (para comparar las masas de los elementos que se pierden por vientos debido a la rama horizontal de las estrellas gigantes), vientos estelares de estrellas masivas, y SNs de colapso de núcleo usando los cálculos de nucleosíntesis de Marigo (2001) y Portinari et al. (1998). Los elementos H, He, C, N, O, Ne, Mg, Si y Fe son trazados individualmente, mientras que para $\mathrm{Ca}$ y $\mathrm{S}$ se suponen cocientes de masa relativos fijos al Si de 0.094 y 0.65, respectivamente (Wiersma et al., 2009b). Además, se computa la pérdida de masa y energía debido a SNIa.

La pérdida de masa por partículas estelares es distribuída sobre las partículas vecinas del kernel $\mathrm{SPH}$, aunque fijando la masa de las partículas gaseosas igual al valor inicial constante, $m_{\mathrm{g}}$. Cada vecino SPH que está separado una distancia $r_{k}$ de una partícula estelar con longitud de suavizado $h$ recibe una fracción $\frac{m_{\mathrm{g}}}{\rho_{k}} W\left(r_{k}, h\right) / \sum_{i} \frac{m_{\mathrm{g}}}{\rho_{i}} W\left(r_{i}, h\right)$ de la masa perdida durante el intervalo temporal de integración, donde $W$ es el kernel SPH y la suma es sobre todos los vecinos SPH.

En Wiersma et al. (2009b) se usan las masas de las partículas de gas en lugar de la masa inicial constante de las partículas de gas cuando se computan sus pesos. El problema con este tratamiento es que las partículas gaseosas que son más masivas que sus vecinas, debido a una reciente acreción de masa por feedback estelar, tienen más peso y entonces se vuelven aún más masivas en relación a sus vecinas. Se encuentra que este proceso desmedido puede causar que una pequeña fracción de partículas terminen con masas que exceden la masa inicial de la partícula. La fracción de partículas muy masivas es siempre baja, porque las partículas masivas son típicamente ricas en metales y se convierten en estrellas relativamente rápido. Sin embargo, no es deseable dirigir la pérdida de masa 
preferencialmente a las partículas de gas relativamente masivas. Entonces, este sesgo se remueve fijando la masa inicial de partícula en lugar de tomar la actual masa de partículas, efectivamente sacando la dependencia con la masa de partícula gaseosa fuera de la ecuación de la pérdida de masa estelar.

También se tiene en cuenta la transferencia de momento y energía asociada con la transferencia de masa de partículas estelares a gaseosas. Nos referimos aquí al momento y energía relacionados a las diferentes velocidades entre las partículas estelares y las partículas gaseosas receptoras, además de las asociadas con los procesos propios de pérdida de masa (como, por ejemplo, los vientos de $\mathrm{SNs}$ ). Luego de ajustar las velocidades de las partículas gaseosas receptoras para que se conserve el momento, la conservación de la energía se alcanza al ajustar las entropías. La transferencia de momento y energía puede, por ejemplo, jugar un rol si la velocidad diferencial entre las componentes estelar y gaseosa es similar o mayor a la velocidad del sonido en el gas, aunque se debe tener en mente que el cambio en la masa de la partícula gaseosa durante un tiempo de enfriamiento es típicamente pequeña.

Al igual que en Wiersma et al. (2009b), las abundancias usadas para evaluar las tasas de enfriamiento radiativo son computadas como el cociente entre la densidad de masa de un elemento sobre la densidad de gas total, donde ambos se calculan usando el formalismo SPH. Las partículas estelares heredan las abundancias suavizadas de las partículas de gas procedentes y se toman en cuenta para computar su tiempo de vida. El uso de abundancias suavizadas SPH, en lugar de fracciones de masa de los elementos almacenados en cada partícula, es consistente con el formalismo SPH. Esto ayuda a aliviar los síntomas que producen la falta de mezclado de metales que ocurren cuando los metales se fijan en las partículas. Sin embargo, esto no resuelve el problema de subestimación del mezcaldo de metales inherente a la técnica SPH. La implementación de coeficientes de difusión efectiva puede ser usada para resolver esto, pero aquí se opta por no hacerlo dado que estos coeficientes permanecen desconocidos para el ISM y el IGM.

La tasa de SNIa por unidad de masa estelar está dada por

$$
\dot{N}_{\mathrm{SNIa}}=v \frac{e^{-t / \tau}}{\tau},
$$

donde $v$ es el número total de SNIa por unidad de masa estelar y $\exp (-t / \tau) / \tau$ es la función distribución del tiempo de decaimiento empírico normalizado. Se fija $\tau=2$ Gyr y $v=2 \times 10^{-3} \mathrm{M}_{\odot}{ }^{-1}$. En cada paso de tiempo en la que la pérdida de masa es evaluada, las partículas estelares transfieren masa y energía asociada a la eyecta de SNIa a sus vecinas. Se utilizan los yields de SNIa del modelo W7 de Thielemann et al. (2003). El feedback energético de SNIa es implementado de manera idéntica al modelo de feedback estelar usando el modelo de feedback térmico estocástico de Dalla Vecchia \& Schaye (2012), considerando $\Delta T=10^{51}$ erg por SNIa, descrito a continuación.

\section{Feedback energético de formación estelar}

Las estrellas pueden inyectar energía y momento al ISM a través de vientos estelares, radiación y SNs. Estos procesos son particularmente importantes para estrellas masivas y, por lo tanto, de corta vida. Si la formación estelar es suficientemente fuerte, el feedback asociado puede generar vientos galácticos de gran escala (ejemplo, Veilleux et al., 2005).

Las simulaciones cosmológicas hidrodinámicas tradicionalmente se han esforzado por hacer un feedback estelar tan eficiente como es requerido para igualar las observaciones de masas de galaxias, tamaños, tasas de eyección y otras observaciones. Si la energía es inyectada térmicamente, tiende a ser radiada rápidamente. Este problema de "sobreenfriamiento" es típicamente atribuido a la falta de resolución numérica. Si la simulación no contiene nubes densas y frías, entonces la formación estelar no es suficientemente grumosa y el feedback energético se distribuye suavemente en demasía. Más aún, dado que las nubes frías reales contienen grandes fracciones de masa del ISM, simulaciones sin 
la fase fría interestelar sobreestiman la densidad de la fase tibia difusa y, así, sobreestiman su tasa de enfriamiento.

Mientras que estos factores pueden contribuir a un problema, Dalla Vecchia \& Schaye (2012) argumentan que el hecho de que la energía se distribuya sobre demasiada masa puede ser un problema más fundamental. Para una IMF estándar hay $1 \mathrm{SN}$ por $100 \mathrm{M}_{\odot}$ de masa SSP y, en realidad, toda la energía mecánica asociada es inicialmente depositada en unas cuantas masas solares de eyección, llevando a una alta temperatura inicial (ejemplo, $\sim 2 \times 10^{8} \mathrm{~K}$ si se depositan $10^{51}$ erg en $10 \mathrm{M}_{\odot}$ de gas). En contraste, en simulaciones SPH que distribuyen la energía producida por una partícula estelar sobre las vecinas SPH, el cociente de la masa calentada sobre la masa de la SSP será mayor que la unidad. Esta disparidad en el cociente de masas implica que la temperatura máxima del calentamiento de gas directo es mucho menor en realidad, y entonces su tiempo de enfriamiento radiativo es mucho más corto. Debido a que el cociente entre la masa de partículas SPH y la de partículas estelares es independiente de la resolución, a primer orden este problema es independiente de la resolución. A segundo orden, tener una mayor resolución ayuda, dado que el feedback térmico puede ser efectivo en generar una eyección si el tiempo de enfriamiento es mayor comparado al tiempo de cruce correspondiente a la velocidad del sonido (sound-crossing) sobre un elemento de resolución, y el último decrece con el incremento de la resolución (sólo como $m_{\mathrm{g}}^{1 / 3}$ ).

Así, los modelos de subgrilla son necesarios para generar vientos galácticos en simulaciones cosmológicas de gran volumen. El tipo de prescripción que se utiliza para las simulaciones EAGLE es el tomado de Dalla Vecchia \& Schaye (2012) y adopta un feedback térmico estocástico. Al hacer que el feedback sea estocástico, se controla la cantidad de energía por evento de feedback incluso si se fija la energía media inyectada por unidad de masa de estrellas formadas. Se especifica el salto en temperatura de las partículas gaseosas receptoras del feedback energético, $\Delta T$, y se usa la fracción de la cantidad total de energía de colapso de núcleo por unidad de masa estelar que es inyectada en promedio, $f_{\text {th }}$, para determinar la probabilidad de que un vecino SPH de partícula estelar joven sea calentada. Se realiza esta operación sólo una vez, cuando la partícula estelar alcanza la edad de $3 \times 10^{7} \mathrm{yr}$, que corresponde al máximo tiempo de vida de estrellas que explotan en este tipo de SN.

El valor $f_{\text {th }}=1$ corresponde al valor esperado de la energía inyectada de $8.73 \times 10^{15} \mathrm{erg} \mathrm{g}^{-1}$ de masa estelar formada, la cual corresponde a la energía disponible para las SNs de colapso de núcleo para una IMF de Chabrier si se supone $10^{51}$ erg por SN y que las estrellas con masas de 6 $100 \mathrm{M}_{\odot}$ exploten. Si $\Delta T$ es suficientemente alta, entonces las pérdidas térmicas iniciales (numéricas espúreas) serán pequeñas y se podrá controlar la eficiencia total del feedback usando $f_{\text {th }}$. La libertad es justificada, dado que habrá pérdidas radiativas físicas en la realidad que no se podrán predecir adecuadamente para el ISM. Más aún, debido a que las verdaderas pérdidas radiativas dependen de las condiciones físicas, se elige variar $f_{\text {th }}$ con las propiedades locales relevantes del gas.

$\mathrm{Al}$ considerar el cociente del tiempo de enfriamiento sobre el tiempo sound-crossing sobre un elemento de resolución, Dalla Vecchia \& Schaye (2012) derivaron la densidad máxima para la que el feedback térmico pueda ser eficiente,

$$
n_{\mathrm{H}, t_{c}} \sim 10 \mathrm{~cm}^{-3}\left(\frac{T}{10^{7.5} \mathrm{~K}}\right)^{3 / 2}\left(\frac{m_{\mathrm{g}}}{10^{6} \mathrm{M}_{\odot}}\right)^{-1 / 2},
$$

donde $T>\Delta T$ es la temperatura luego de la inyección de energía y se usa $\Delta T=10^{7.5} \mathrm{~K}$. Esta expresión supone que la tasa de enfriamiento radiativo es dominado por emisión libre-libre y entonces sobreestima el valor de $n_{\mathrm{H}, t_{c}}$ cuando $T<<10^{7} \mathrm{~K}$. En estas simulaciones, algunas estrellas se forman, de hecho, de gas que excede el valor crítico $n_{\mathrm{H}, t_{c}}$, particularmente en galaxias masivas. Aunque la densidad del gas en que las estrellas inyectan su energía generalmente sea menor que el gas de donde las partículas estelares se formaron, dado que las partículas estelares se mueven relativas al gas durante $3 \times 10^{7}$ yr entre la formación estelar y el feedback, esto significa que las estrellas que se forman en gas de alta densidad tendrán pérdidas radiativas que exceden aquéllas que ocurren en una simulación que 
tiene la resolución y la física requerida para resolver la escala pequeña de la estructura del ISM. Dado que se calibra la cantidad total de energía que es inyectada por unidad de masa estelar, esto implica que podríamos sobreestimar la cantidad requerida de feedback energético. En el rango masivo de masa estelar, el feedback de AGN controla la eficiencia de formación de las galaxias en la simulación. Si las pérdidas radiativas del feedback estelar son sobreestimadas, esto podría potencialmente causar una sobreestimación en la eficiencia requerida de feedback de AGN.

La densidad crítica, $n_{\mathrm{H}, t_{c}}$, incrementa con la resolución numérica, pero también con el salto en temperatura, $\Delta T$. Se podría entonces reducir las pérdidas térmicas iniciales incrementando $\Delta T$. Sin embargo, para una cantidad fija de energía por unidad de masa estelar (es decir, para un valor fijo de $f_{\text {th }}$ ), la probabilidad de que una partícula estelar particular genere feedback es inversamente proporcional a $\Delta T$. Dalla Vecchia \& Schaye (2012) muestran que, para el caso de partículas de igual masa, el valor esperado para el número de partículas gaseosas calentadas por partícula estelar es

$$
\left\langle N_{\text {heat }}\right\rangle \approx 1.3 f_{\text {th }}\left(\frac{\Delta T}{10^{7.5} \mathrm{~K}}\right)^{-1}
$$

para una IMF de Chabrier y sólo teniendo en cuenta la energía por SN. Entonces, usando $\Delta T>>$ $10^{7.5} \mathrm{~K}$ o $f_{\text {th }}<<1$ implicaría que la mayoría de las partículas estelares no inyectan energía por SN de colapso de núcleo en sus alrededores, lo que lleva a un muestreado pobre del ciclo de feedback. Entonces se mantiene el salto en temperatura $\Delta T=10^{7.5} \mathrm{~K}$. Aunque la implementación estocástica permite un feedback térmico eficiente sin la necesidad de apagar el enfriamiento, las pérdidas térmicas son poco probables de converger con la resolución numérica de EAGLE. Entonces, la re-calibración de $f_{\text {th }}$ puede llegar a ser necesaria cuando la resolución sea cambiada.

\section{Dependencia con las propiedades locales del gas}

Se espera que las verdaderas pérdidas térmicas en el ISM incrementen cuando la metalicidad sea lo suficientemente alta como para que los enfriamientos por metalicidad se vuelvan importantes. Para temperaturas de $10^{5} \mathrm{~K}<T<10^{7} \mathrm{~K}$ esto sucede cuando $Z \gtrsim 10^{-1} \mathrm{Z}_{\odot}$ (Wiersma et al., 2009a). Aunque la dependencia exacta con la metalicidad no puede ser predicha sin un completo conocimiento de las condiciones físicas del ISM, se puede capturar la transición cualitativa esperada por los metales al hacer a $f_{\text {th }}$ una función de la metalicidad,

$$
f_{\mathrm{th}}=f_{\mathrm{th}, \min }+\frac{f_{\mathrm{th}, \max }-f_{\mathrm{th}, \min }}{1+\left(\frac{Z}{0.1 Z_{\odot}}\right)^{n_{Z}}},
$$

donde $Z_{\odot}=0.0127$ es la metalicidad solar y $n_{Z}>0$. Notar que $f_{\text {th }}$ se acerca asintóticamente a $f_{\text {th,max }}$ y a $f_{\text {th,min }}$ para $Z<<0.1 \mathrm{Z}_{\odot}$ y $Z>>0.1 \mathrm{Z}_{\odot}$, respectivamente.

Dado que la metalicidad decrece al aumentar el redshift a masa estelar fija, la motivación física de la dependencia con la metalicidad tiende a hacer al feedback relativamente más eficiente a alto redshift. Sin embargo, se encuentra que una dependencia puramente con la metalicidad resulta en galaxias muy compactas, lo que indica que el feedback es muy ineficiente a altas densidades. Esto no es inesperado; el problema se resuelve al aumentar la resolución.

Se encuentra entonces deseable compensar por las excesivas pérdidas térmicas iniciales a altas densidades agregando una dependencia con la densidad a $f_{\text {th }}$,

$$
f_{\mathrm{th}}=f_{\mathrm{th}, \min }+\frac{f_{\mathrm{th}, \text { max }}-f_{\mathrm{th}, \min }}{1+\left(\frac{Z}{0.1 \mathrm{Z}_{\odot}}\right)^{n_{\mathrm{Z}}}\left(\frac{n_{\mathrm{H}, \mathrm{birh}}}{n_{\mathrm{H}, 0}}\right)^{-n_{n}}},
$$

donde $n_{\mathrm{H} \text {,birth }}$ es la densidad heredada de la partícula estelar (Crain et al., 2015). Entonces, $f_{\text {th }}$ incrementa con la densidad a metalicidad fija, mientras que sigue respetando los originales valores asintóticos. Los valores que $n_{n}$ y $n_{\mathrm{H}, 0}$ toman pueden verse en la Tabla 2.3 (se supone $n_{Z}=n_{n}$ ). La 
dependencia con la densidad de $f_{\text {th }}$ puede tener una interpretación física. Por ejemplo, densidades medias altas en escalas de $10^{2}-10^{3}$ pc pueden resultar en formación estelar más grumosa, lo que reduce las pérdidas térmicas. Sin embargo, se enfatiza que la motivación principal es contrarrestar el exceso en las pérdidas térmicas en altas densidades del ISM que pueden ser atribuidas al límite de resolución.

\section{Agujeros negros y feedback de AGN}

En las simulaciones EAGLE, el feedback por acreción de BHs supermasivos reduce o extingue la formación estelar en galaxias masivas, moldea los perfiles de gas en las regiones internas de sus halos huéspedes, y regula el crecimiento de los BHs. Los modelos suelen hacer la distinción entre feedback de BH en modo "quasar" y modo "radio", donde el primero ocurre cuando el BH acreta eficientemente (con valores similares o superiores a la tasa de acreción de Eddington), e inyecta energía al medio en forma de viento nuclear caliente, mientras que el modo radio opera cuando la tasa de acreción es baja en comparación con la tasa de Eddington y la energía es inyectada por medio de flujos (o jets) relativísticos (Sijacki et al., 2008). Debido a que las simulaciones cosmológicas carecen de la resolución para distinguir estos mecanismos adecuadamente, y se trata de limitar el número de canales de feedback para tener buen acuerdo con las observaciones de interés, se elige implementar un solo modo de feedback de AGN con una eficiencia fija. La energía inyectada térmicamente en la ubicación del BH es proporcional a la tasa de acreción de gas. Esta implementación puede entonces ser más cercana al proceso referido como modo quasar. Este método lleva a un excelente acuerdo con las observaciones de grupos y cúmulos tanto en óptico como en rayos X (McCarthy et al., 2011; Le Brun et al., 2014).

La implementación consiste en dos partes. Por un lado, se utilizan prescripciones para incorporar una semilla de $\mathrm{BH}$ central en las galaxias de baja masa y su crecimiento por medio de la acreción de gas y fusiones. Esto se hace para galaxias cuya masa total sea mayor a $10^{10} h^{-1} \mathrm{M}_{\odot}$ y que aún no contengan un BH en sus centros (Springel et al., 2005). La tasa en la que los BHs acretan gas dependerá de la masa del $\mathrm{BH}$, la densidad y temperatura locales, la velocidad relativa del $\mathrm{BH}$ en el gas, y el momento angular del gas con respecto al $\mathrm{BH}$. Las fusiones de los $\mathrm{BHs}$ dependerán de la distancia entre ellos y de las velocidades circulares que estos objetos tengan (para más detalle sobre el crecimiento de los BHs en las simulaciones EAGLE, ver S15). Por otro lado, se utiliza una prescripción para el feedback energético, explicado a continuación.

El feedback de AGN es implementado térmica y estocásticamente, de manera análoga al feedback energético de la formación estelar. La inyección de energía es $\epsilon_{\mathrm{f}} \epsilon_{\mathrm{r}} \dot{m}_{\mathrm{accr}} c^{2}$, donde $\epsilon_{\mathrm{f}}=0.15$ es la fracción de energía radiada que está acoplada al ISM, $\epsilon_{\mathrm{r}}=0.1$ es la eficiencia radiativa del disco de acreción, $\dot{m}_{\text {accr }}$ es la tasa de acreción del BH, y $c$ es la velocidad de la luz. Como es el caso de la eficiencia de feedback estelar, $f_{\mathrm{th}}$, el valor $\epsilon_{\mathrm{f}}$ debe ser calibrado por la relación entre la masa del BH y la masa estelar. Como se demostró en Booth \& Schaye (2010), el valor de $\epsilon_{\mathrm{f}}$ sólo afecta las masas de los BHs, las cuales son inversamente proporcionales a $\epsilon_{\mathrm{f}}$. En particular, la tasa de eyecciones generadas por el AGN, y entonces también el factor por el que la formación estelar es reducida, es altamente insensible a $\epsilon_{\mathrm{f}}$ siempre y cuando éste sea distinto de cero. Esto puede ser explicado por la auto-regulación: la tasa de acreción del BH se ajusta hasta que la tasa a la que la energía es inyectada es suficiente para que las eyecciones balanceen las acreciones. Los valores usados para la eficiencia de AGN, $\epsilon_{\mathrm{f}}=0.15$ y $\epsilon_{\mathrm{r}}=0.1$, implican que una fracción $\epsilon_{\mathrm{f}} \epsilon_{\mathrm{r}}=0.015$ de la energía de masa acretada en reposo vuelve al ISM local. Como es el caso para el feedback estelar, el valor requerido dependerá de las pérdidas radiativas del ISM, las cuales pueden depender de la resolución y de la manera precisa en la que la energía es inyectada. Dado que no se espera que los metales dominen las pérdidas radiativas a altas temperaturas asociadas a feedback de AGN, no se implementa una dependencia con la metalicidad.

Cada BH tiene un "reservorio" de feedback energético, $E_{\mathrm{BH}}$. Luego de un paso de tiempo $\Delta t$, se añade $\epsilon_{\mathrm{f}} \epsilon_{\mathrm{r}} \dot{m}_{\mathrm{accr}} c^{2} \Delta t$ a este reservorio. Si el BH ha almacenado suficiente energía para calentar al 
menos $n_{\text {heat }}$ partículas de masa $m_{\mathrm{g}}$, entonces el BH calienta estocásticamente cada uno de los vecinos $\mathrm{SPH}$ al incrementar su temperatura por $\Delta T_{\mathrm{AGN}}$. Por cada vecino, la probabilidad de calentarse es

$$
P=\frac{E_{\mathrm{BH}}}{\Delta \epsilon_{\mathrm{AGN}} N_{\mathrm{ngb}}\left\langle m_{\mathrm{g}}\right\rangle},
$$

donde $\Delta \epsilon_{\mathrm{AGN}}$ es el cambio en la energía interna por unidad de masa correspondiente al incremento de temperatura, $\Delta T_{\mathrm{AGN}}, N_{\mathrm{ngb}}$ es el número de vecinos gaseosos del $\mathrm{BH}$, y $\left\langle m_{\mathrm{g}}\right\rangle$ es la masa promedio de estos vecinos. La $E_{\mathrm{BH}}$ se reduce por el valor esperado de la energía inyectada. Usamos $n_{\text {heat }}=1 \mathrm{y}$ limitamos el paso de tiempo de los BHs tal que $P<0.3$.

El parámetro más importante para el feedback de AGN es el incremento en temperatura, $\Delta T_{\mathrm{AGN}}$. Valores mayores harán que eventos de feedback individuales sean más energéticos, generalmente como resultado de pérdidas radiativas menores en el ISM. Sin embargo, valores altos también harán que el feedback sea más intermitente. Debido a que la densidad del gas alrededor del BH tiende a incrementar con la resolución, se encuentra que se necesita incrementar $\Delta T$ cuando se incrementa la resolución. De manera similar, dado que la densidad del gas que rodea el $\mathrm{BH}$ alcanza valores que son más altos que para el gas SF típico, se requieren mayores saltos en temperatura por feedback de AGN que para feedback estelar.

\subsubsection{Simulaciones usadas en este trabajo}

Para este trabajo, usamos dos de las simulaciones EAGLE. Por un lado, usamos la simulación de referencia, llamada aquí como Ref-L100N1504, la cual tiene un volumen de $100^{3} \mathrm{cMpc}^{3}$ (donde cMpc denota Mpc comóviles ${ }^{3}$ ).

Por otro lado, usamos la simulación recalibrada de alta resolución, referida como Recal-L25N752. Los parámetros de ambas simulaciones pueden encontrarse en la Tabla 2.2.

\begin{tabular}{lrr}
\hline Parámetro & Recal-L25N752 & Ref-L100N1504 \\
\hline$L(\mathrm{cMpc})$ & 25 & 100 \\
$N$ & $752^{3}$ & $1504^{3}$ \\
$m_{\mathrm{g}}\left(\mathrm{M}_{\odot}\right)$ & $2.26 \times 10^{5}$ & $1.81 \times 10^{6}$ \\
$m_{\mathrm{DM}}\left(\mathrm{M}_{\odot}\right)$ & $1.21 \times 10^{6}$ & $9.70 \times 10^{6}$ \\
$\epsilon_{\text {prop }}(\mathrm{pkpc})$ & 0.35 & 0.70 \\
\hline
\end{tabular}

Tabla 2.2: Parámetros principales de las simulaciones EAGLE usadas en este trabajo. $L$ es el lado comóvil de la caja, $N$ el número de partículas de materia oscura (hay inicialmente igual número de partículas bariónicas), $m_{\mathrm{g}}$ es la masa inicial de las partículas bariónicas, $m_{\mathrm{DM}}$ es la masa inicial de las partículas de materia oscura, y $\epsilon_{\text {prop }}$ es la longitud máxima del suavizado (o softening) en unidades físicas.

EAGLE ha probado tener éxito en una variedad significativa de comparaciones. Varios trabajos han mostrado que la simulación Ref-L100N1504 reproduce las propiedades observadas de las galaxias como la evolución de la función de masa estelar desde $z=0$ a $z=4$, la secuencia principal de galaxias (Furlong et al., 2015), la distribución de colores de las galaxias (Trayford et al., 2015; Wright et al., 2019), y el contenido gaseoso de las galaxias a una dada masa estelar (Lagos et al., 2015, 2016; Bahé et al., 2016; Crain et al., 2017). Más relevante para este trabajo, la MZR de estrellas y gas resuelta (Trayford \& Schaye, 2019), así como también los gradientes de metalicidad (S15, Tissera et al. 2019), son bien reproducidas para galaxias con masa $M_{\star} \geq 10^{10} \mathrm{M}_{\odot}$ para Ref-L100N1504.

A bajas masas estelares $\left(M_{\star} \leq 10^{10} \mathrm{M}_{\odot}\right)$, las desviaciones con las observaciones se vuelven más importantes. La simulación Recal-L25N752 alivia este problema, generando acuerdo hacia masas

\footnotetext{
${ }^{3}$ Las unidades comóviles son aquéllas que no cambian con la expansión del universo. La diferencia entre las unidades comóviles y las físicas (referidas a veces con un prefijo $p$ por physical) es que para pasar de unas a otras se necesita multiplicar o dividir por el factor de escala $a$.
} 
estelares de $10^{9} \mathrm{M}_{\odot}$ (ver S15, De Rossi et al. 2017). De esta forma, el uso simultáneo de las simulaciones presentadas en la Tabla 2.2 permiten tener una buena estadística para masas estelares de $\geq 10^{10} \mathrm{M}_{\odot}$ (mayormente de la simulación Ref-L100N1504), y empujar el límite a $10^{9} \mathrm{M}_{\odot}$, gracias a la simulación Recal-L25N752, con la confianza de que la MZR observada es bien reproducida en este rango de masa estelar.

En particular, el fuerte feedback de la simulación Recal-L25N752 tiende a remover más metales del ISM que lo que se ve para Ref-L100N1504, lo cual constituye parcialmente la razón por la cual las metalicidades de la primera simulación son menores para galaxias de masas estelares $<10^{10} \mathrm{M}_{\odot}$. S15 discutieron esto y mostraron que versiones de alta resolución de Ref-L100N1504 (es decir, misma física de subgrilla que Ref-L100N1504 pero con número de partículas y volumen igual a RecalL25N752) produce metalicidades del gas que son $\sim 0.2$ dex mayores que Recal-L25N752 a masas estelares de $\approx 10^{8} \mathrm{M}_{\odot}$. Aunque el punto cero de la relación es ligeramente diferente entre estas dos simulaciones, las pendientes está en acuerdo una vez que se estudian las metalicidades de forma relativa a su masa estelar (ver Sección 4.5).

El modelo físico de subgrilla de EAGLE impone un límite a la temperatura del gas que depende de su densidad para prevenir fragmentación espúrea debido a la resolución finita de la simulación. Por lo tanto, EAGLE no modela la fase fría de gas, lo que hace que el ISM de las galaxias sea más homogéneo (o menos grumoso) que lo observado en galaxias reales, llevando a estrellas y polvo a ser, en principio, igualmente mezclados de lo que serían si la fase de gas frío estuviera incluida. A pesar de esta limitación, al incrementar la resolución de la simulación, aunque manteniendo el límite de temperatura mencionado, el ISM muestra un incremento en los niveles de grumosidad que no es debido al cálculo numérico sino que es un efecto físico (Trayford \& Schaye, 2019; Trayford et al., 2020). En EAGLE, las estrellas se forman en estos grumos que tienen extensión $\leq 1 \mathrm{kpc}$, lo que es mayor que las nubes moleculares típicas en el Universo local. Como resultado, se espera que los metales estén más mezclados en el ISM comparado con lo que se esperaría si se generara grumosidad. Una consecuencia de esto podría ser una menor variación de metalicidades sobre una galaxia, pero dado que aquí nos interesa medir perfiles de metalicidad en intervalos radiales de $\approx$ $1 \mathrm{kpc}$, no encontramos grandes limitaciones. Las observaciones actuales que utilizan la técnica de IFS para restringir los gradientes de metalicidad tienen una resolución espacial similar a la que se consigue combinando las simulaciones Recal-L25N752 para galaxias con $M_{\star}<10^{10} \mathrm{M}_{\odot}$ y RefL100N1504 para galaxias más masivas $(500-1000$ pc para su mediana en redshift; por ejemplo, van de Sande et al. 2019).

\subsubsection{Calibraciones de las simulaciones EAGLE y tipos de convergencia}

Como se mencionó anteriormente, debido a que se desconoce exactamente la expresión para la eficiencia del feedback, es necesario calibrar las simulaciones para que los parámetros libres usados puedan ser determinados. En particular, nos interesan 4 de estos parámetros libres, cuyos valores resultan modificados al cambiar la resolución de las simulaciones. Ellos son: la densidad característica, $n_{\mathrm{H}, 0}$, y la pendiente de la ley de potencia, $n_{n}$, de la densidad dependiente de la energía del feedback producto de la formación estelar; el parámetro que controla la sensibilidad de la tasa de acreción de los BHs con respecto al momento angular del gas que los rodea, $C_{\mathrm{visc}}$; y el incremento en temperatura que regula el feedback de AGN, $\Delta T_{\mathrm{AGN}}$. Los valores numéricos que estos parámetros toman dependiendo de la simulación pueden verse en la Tabla 2.3.

Las restricciones observacionales que se utilizan para calibrar las simulaciones EAGLE se describen a continuación. La función de masa estelar de galaxias GSMF a $z=0.1$ muestra la cantidad de galaxias que hay de determinada masa estelar (Figura 2.6). La relación entre la masa estelar y la masa del halo de las galaxias a $z \sim 0$ relaciona la cantidad de materia bariónica y de DM de las galaxias (Figura 2.7). La relación entre los radios a mitad de masa estelar y la masa estelar de las galaxias que están formando estrellas a tasas normales a $z=0.1$, la cual da información de los tama- 
ños de las galaxias (Figura 2.8). Por último, se calibra la relación entre la masa de los BHs centrales supermasivos y la masa estelar de las galaxias (Figura 2.9). Todas estas relaciones se muestran para las simulaciones calibradas comparadas tanto con las correspondientes observaciones como con los resultados de la simulación AGNdT9-L050N0752, la cual es una simulación con de $50 h^{-1} \mathrm{Mpc}$ de lado y con $2 \times 752^{3}$ número de partículas, cuya corrida se debe al intento de mejorar el acuerdo con las observaciones de galaxias de alta masa (siendo los valores de sus parámetros libres $n_{\mathrm{H}, 0}=0.67$, $n_{n}=2 / \ln 10, C_{\mathrm{visc}}=2 \pi \times 10^{2}$ y $\left.\Delta T_{\mathrm{AGN}}=10^{9}\right)$.

\begin{tabular}{lrr}
\hline Parámetro & Recal-L25N752 & Ref-L100N1504 \\
\hline$n_{\mathrm{H}, 0}\left(\mathrm{~cm}^{-3}\right)$ & 0.25 & 0.67 \\
$n_{n}$ & $1 / \ln 10$ & $2 / \ln 10$ \\
$C_{\mathrm{visc}}$ & $2 \pi \times 10^{3}$ & $2 \pi$ \\
$\Delta T_{\mathrm{AGN}}(\mathrm{K})$ & $10^{9}$ & $10^{8.5}$ \\
\hline
\end{tabular}

Tabla 2.3: Valores de los parámetros libres de las simulaciones EAGLE que se modifican según la simulación. $n_{\mathrm{H}, 0}$ y $n_{n}$ controlan, respectivamente, la densidad característica y la pendiente de ley de potencia de la densidad dependiente de la energía de feedback de formación estelar, $C_{\text {visc }}$ es el parámetro que controla la sensibilidad de la tasa de acreción del $\mathrm{BH}$ con respecto al momento angular del gas, y $\Delta T_{\mathrm{AGN}}$ es el incremento de temperatura del gas durante el feedback de AGN.

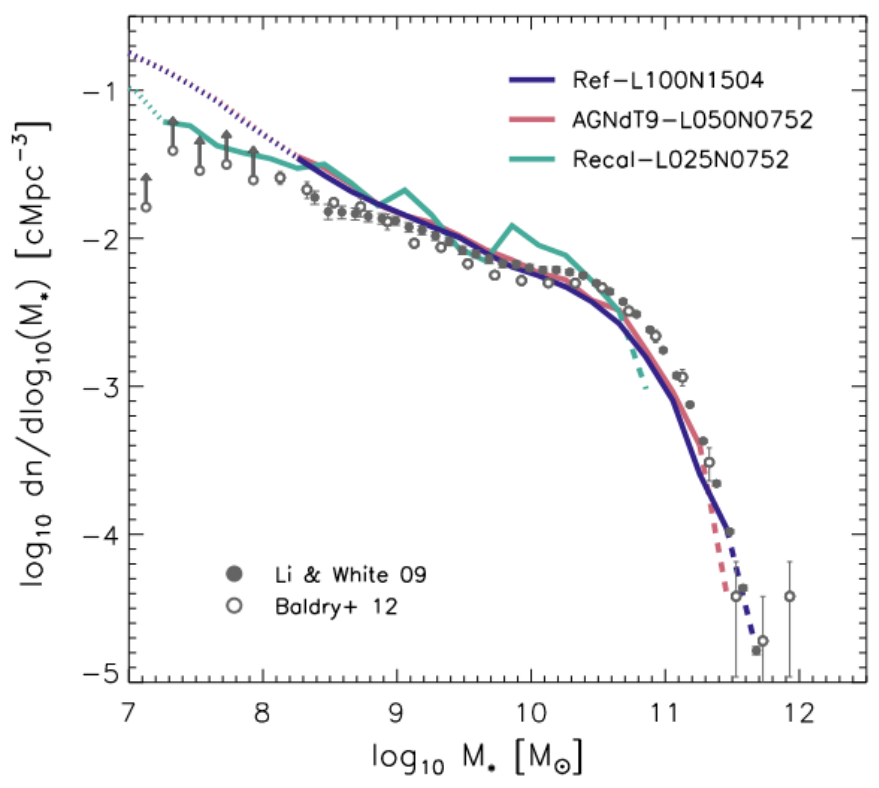

Figura 2.6: GSMF a $z=0.1$ para las simulaciones EAGLE Ref-L100N1504 (azul), AGNdT9-L050N0752 (rojo), y Recal-L25N752 (verde azulado). Las curvas cambian de sólida a trazos en el extremo masivo y cuando hay menos de 10 objetos por intervalo de masa estelar. En el extremo poco masivo, las curvas se vuelven punteadas cuando la masa estelar cae por debajo de las 100 partículas bariónicas. Los símbolos muestran las mediciones del relevamiento GAMA (círculos vacíos, $z<0.06$; Baldry et al. 2012) y del SDSS (círculos llenos, $z \sim 0.07$; Li \& White 2009). El modelo de alta resolución Recal-L25N752 produce mayor ruido debido al pequeño tamaño de su caja. Los modelos de resolución intermedia subestiman ligeramente la densidad numérica de galaxias en la rodilla de la función de masa, y ligeramente sobreestiman la abundancia a $M_{\star} \sim$ $10^{8.5} \mathrm{M}_{\odot}$. La densidad numérica de las galaxias está en buen acuerdo con los datos observacionales a $\lesssim 0.2$ dex. Figura adoptada de S15.

Algo importante a remarcar es que la mayoría de las veces no resulta sencillo encontrar observables que permitan calibrar las simulaciones para que generen una población de galaxias realista. Una 
forma de proceder, la cual es una muy buena práctica para aprender sobre la física de la formación galáctica, es correr múltiples simulaciones variando los modelos de subgrilla. En este sentido, resulta extremadamente útil contar con múltiples prescripciones calibradas con los mismos observables. Crain et al. (2015) analizan 4 variaciones en la prescripción de feedback estelar (una de ellas, la de referencia, presentada en la Sección 2.2.2), las cuales todas reproducen la GSMF a $z \sim 0$, pero sólo la que utiliza el modelo de referencia genera una población de galaxia con tamaños realistas (el resto genera galaxias extremadamente compactas). Así, la calibración de las simulaciones EAGLE no sólo con la GSMF (Figura 2.6) sino también teniendo en cuenta los tamaños de las galaxias (Figura 2.8) permite que las simulaciones que usan el modelo de subgrilla presentado en este Capítulo generen una población realista de galaxias.

Dado que estos observables fueron considerados durante la calibración de los modelos de feedback, no pueden tomarse como predicciones de la simulación. Sin embargo, cabe destacar que no hay control alguno sobre la pendiente de la relación $M_{\mathrm{BH}}-M_{\star}$ y que los tamaños de las galaxias fueron sólo usados para descartar modelos fuertemente discrepantes (es decir, modelos sin una dependencia en la densidad de energía del feedback de la formación estelar). El éxito alcanzado en la comparación con las observaciones no resulta a priori garantizado dado que el costo computacional de las simulaciones hidrodinámicas limita drásticamente el número de pruebas que pueden realizarse y, más importante, debido a que la libertad propia del modelo es limitada.
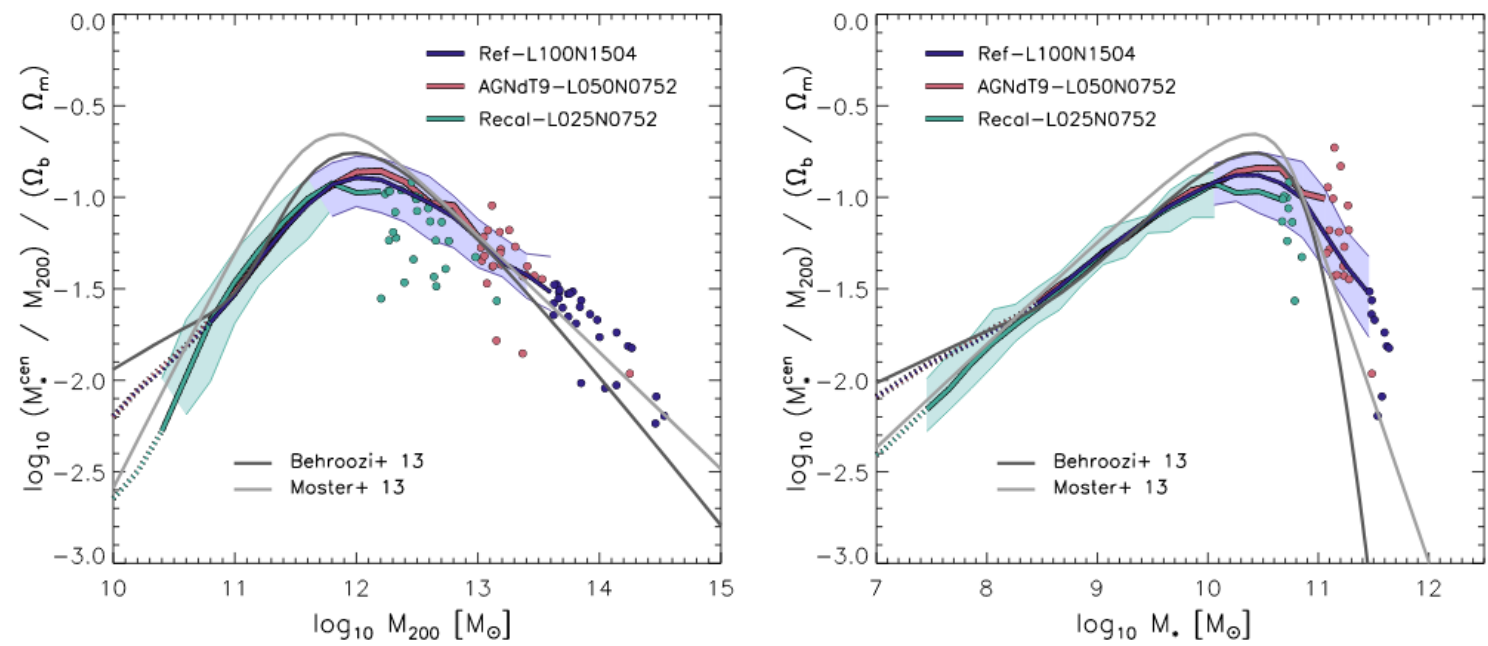

Figura 2.7: Cociente entre la masa estelar y la masa de halo, relativo a la fracción bariónica universal, como función de la masa del halo (panel izquierdo) y a la masa estelar (panel derecho) para galaxias centrales. Las curvas de las simulaciones se vuelven punteadas cuando hay menos de 100 partículas estelares por galaxia. Los círculos llenos muestran galaxias individuales cuando hay menos de 10 galaxias por intervalo. Las regiones sombreadas muestran la dispersión de $1 \sigma$ en las simulaciones. Por claridad sólo se muestra la dispersión en Recal-L25N752 para $M_{\star}<10^{10} \mathrm{M}_{\odot}$ y en Ref-L100N1504 para $M_{\star}>10^{10} \mathrm{M}_{\odot}$. Los resultados de EAGLE están en buen acuerdo con los resultados inferidos de observaciones de Behroozi et al. (2013a, curvas grises oscuras) y Moster et al. (2013, curvas grises claras). La pequeña diferencia entre EAGLE y las observaciones alrededor y sobre el pico de la relación es consistente con la leve subestimación de EAGLE a la rodilla de la GSMF. Figura adoptada de S15.

La necesidad de calibrar la eficiencia del feedback y sus límites asociados al poder predictivo de las simulaciones pone en debate la cuestión de la convergencia numérica. Desde el punto de vista convencional, los modelos de subgrilla deberían ser diseñados para realizar predicciones numéricamente convergentes. Sin embargo, las simulaciones actuales no pueden realizar esto, en todo caso hacen una predicción a priori para algunos de los observables más fundamentales de la población de galaxias. Mientras que es obvio que se debería demandar convergencia para las predicciones que son 
relativamente robustas para la elección del modelo de subgrilla, es menos obvio que algunos de los requerimientos para las observaciones dependan directa y fuertemente de la eficiencia del feedback de subgrilla. Se podría argumentar que, en cambio, sólo se necesita convergencia luego de re-calibrar el modelo de subgrilla. Esto se llama "convergencia débil", en oposición a la "convergencia fuerte" que es obtenida si los resultados no cambian con la resolución cuando el modelo se fija (S15). Si sólo se necesita convergencia débil, entonces las demandas puestas en el modelo de subgrilla se reducen, lo cual tiene dos ventajas. Primero, se obtienen ventajas al incrementar la resolución. La escala de subgrilla puede moverse a lo largo del límite de resolución, por lo que se podría potencialmente modelar la física de manera más fiel si se adopta una resolución mayor. Una segunda ventaja de demandar solamente convergencia débil es que no se deben hacer sacrificios que requieran mejorar la convergencia fuerte y que podrían tener consecuencias indeseables.

Actualmente, las simulaciones que muestrean un volumen significativo tienen escasa resolución y física para predecir las pérdidas radiativas del ISM. La convergencia fuerte puede, sin embargo, ser alcanzada si estas pérdidas son de alguna manera removidas. Ejemplos de esta práctica incluyen la detención temporal del enfriamiento radiativo, y de las fuerzas hidrodinámicas, y hacer que la eficiencia del feedback dependa de la dispersión de velocidad de la DM en lugar de las propiedades locales del gas. Esto puede generar que se necesite introducir nuevos parámetros para controlar cuándo se detienen y reinician los distintos procesos de pérdida. Estos parámetros pueden directamente afectar los resultados buscados, incluyendo el estado del gas que rodea a las galaxias, y también puede reintroducir efectos de resolución. Sin embargo, sobrepasar las pérdidas radiativas en el ISM no es por sí solo suficiente para alcanzar la convergencia fuerte.

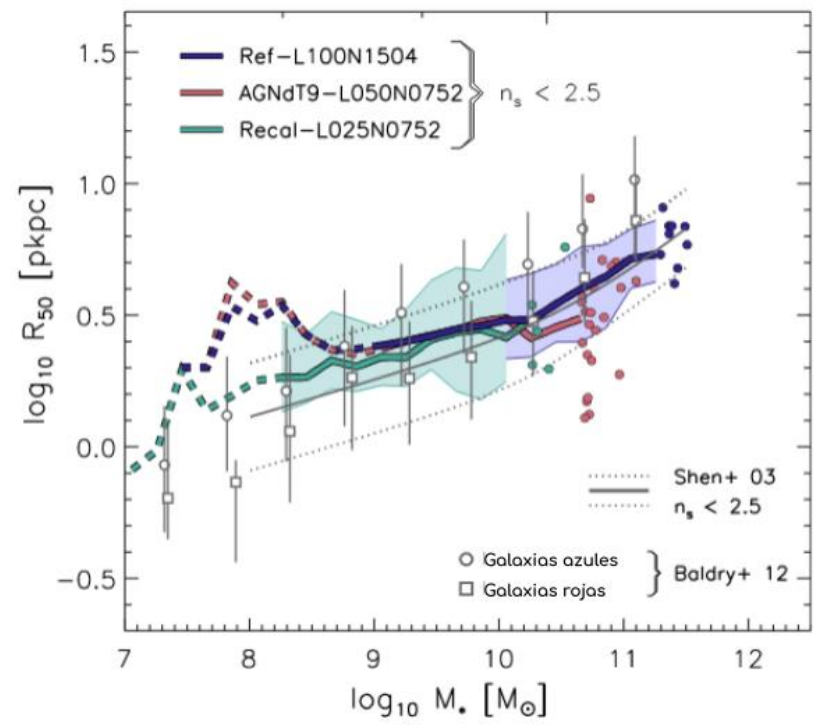

Figura 2.8: Tamaño de las galaxias como función de la masa estelar para galaxias a $z=0.1$ (en escala de pkpc). Las curvas coloreadas muestran la mediana a radio mitad de masa proyectado para las simulaciones y las sombras muestran la dispersión de $1 \sigma$. Por claridad sólo se muestra la dispersión en Recal-L25N752 para $M_{\star}<10^{10} \mathrm{M}_{\odot}$ y en Ref-L100N1504 para $M_{\star}>10^{10} \mathrm{M}_{\odot}$. Las curvas de las simulaciones se vuelven punteadas cuando la resolución desciende del límite de 100 partículas estelares. Cuando hay menos de 10 galaxias por intervalo, los objetos individuales se muestran con círculos llenos. Los modelos son comparados con los radios a mitad de luz de Sérsic de galaxias del SDSS (Shen et al., 2003, la línea gris sólida muestra la mediana y las líneas grises punteadas indican la dispersión de $1 \sigma$ ) y GAMA (Baldry et al., 2012, puntos con barras de error que indican la dispersión de $1 \sigma$, mostrando por separado galaxias azules y rojas). Las simulaciones y Shen et al. (2003) sólo incluyen galaxias del tipo tardío, es decir, con índice de Sérsic $n_{\mathrm{s}}<2.5$. Figura adoptada de S15. 
En la práctica, sin embargo, la distinción entre convergencia débil y fuerte no suele ser clara. Se puede suponer que manteniendo los modelos físicos fijos es equivalente a mantener fijos los parámetros del código y de la subgrilla (aparte de los parámetros numéricos que controlan la resolución), pero esto no es necesariamente el caso debido a la confianza de las prescripciones de subgrilla y a su inhabilidad para resolver las primeras generaciones de estrellas y BHs. Para las prescripciones de subgrilla típicas, la energía, masa y momento involucrados en los eventos individuales de feedback, y el número de eventos de feedback no se mantienen todos fijos cuando la resolución cambia. Estos cambios pueden afectar la eficiencia del feedback. Más aún, para condiciones iniciales cosmológicas, tener mayor resolución implica resolver halos más pequeños, y entonces rastrear progenitores de galaxias a tiempo presente con mayor redshift. Si estos progenitores tienen vientos, esto puede afectar la subsecuente evolución. Más aún, la eficiencia del feedback no puede, en ningún caso, ser predicha desde el principio, incluso cuando la convergencia sea perfecta.

La búsqueda por una convergencia fuerte en las simulaciones que carecen de resolución para modelar el ISM ha llevado a sacrificios significativos, que generalmente involucran la desactivación de aspectos de la hidrodinámica durante el feedback. Sin embargo, hasta que las pérdidas por enfriamiento puedan ser predichas incluso las simulaciones completamente convergentes no serán capaces de predecir las masas estelares y de BHs desde el principio. Debido a esto, las simulaciones EAGLE optan por asegurar una convergencia débil, minimizando los sacrificios mencionados (aunque estas simulaciones muestran una buena convergencia fuerte, S15).

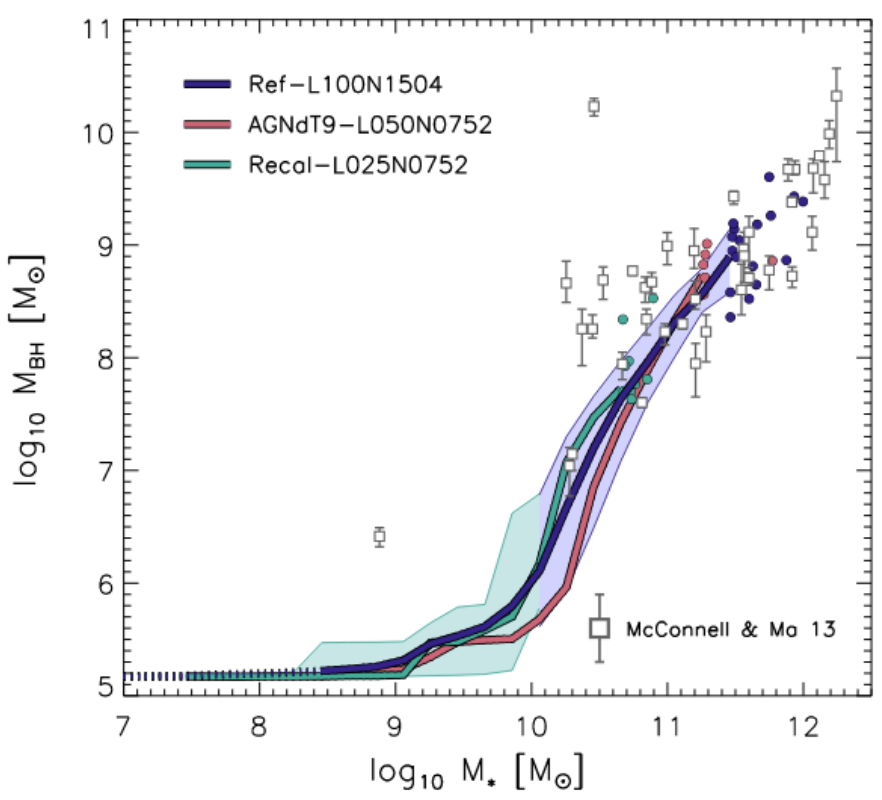

Figura 2.9: La relación entre la masa de los BHs centrales supermasivos de las galaxias y su masa estelar. Las curvas coloreadas muestran la mediana de la relación para las simulaciones y las sombras muestran la dispersión de $1 \sigma$. Cuando hay menos de 10 galaxias por intervalo, los objetos individuales se muestran con círculos llenos. Los puntos con sus barras de error, indicando la dispersión de $1 \sigma$, muestran la compilación de observaciones de McConnell \& Ma (2013). Las simulaciones muestran la masa estelar total (dentro de una apertura 3D de $30 \mathrm{pkpc}$, mientras que las observaciones muestran las masas de los bulbos. Sin embargo, las galaxias observadas fueron seleccionadas por ser del tipo temprano. Las simulaciones están en acuerdo con las observaciones, aunque la dispersión observada sea mayor. Figura adoptada de S15. 


\section{Capítulo 3}

\section{El impacto del Feedback de SN en la Relación Masa-Metalicidad}

La relación masa-metalicidad muestra la relación de la metalicidad del gas como función de la masa estelar de las galaxias. En general, galaxias más masivas presentan también mayor metalicidad. Sin embargo, la forma y normalización de esta relación puede dar información importante sobre distintos procesos físicos que sucedieron en las galaxias. Es decir, eventos de interacciones o fusiones que las galaxias sufren, la eficiencia y cambios en su SFR, y la caída y eyección de material, entre otros procesos, dejan su huella en la MZR.

Aquí nos enfocaremos en cómo los cambios en la descripción del feedback de SN pueden moldear de manera radicalmente distinta la forma de la MZR, y en los efectos que estos cambios producen en la evolución de la MZR. Para ello, haremos uso de distintos modelados del feedback de SN que se describen en la Sección 2.1.3. Presentamos, entonces, las predicciones de la MZR obtenidas para distintas versiones del modelo SAG, tanto a $z=0$ como a alto redshift, comparándola a su vez con distintos datos observados. Los resultados que se muestran en este Capítulo han sido publicados en el trabajo Collacchioni et al. (2018).

A lo largo de este Capítulo, nos referiremos a la abundancia del oxígeno respecto al hidrógeno del medio interestelar de las galaxias del modelo como la metalicidad del gas. Calculamos esta metalicidad como $12+\log (\mathrm{O} / \mathrm{H})$, donde $\mathrm{O} / \mathrm{H}$ es el número de núcleos de oxígeno sobre el número de núcleos de hidrógeno. Esta definición implícita significa que estamos calculando la abundancia a partir de la masa (mass-weighted metalliticy), mientras que en observaciones esta estimación se obtiene a partir de la luminosidad de las regiones HII (luminosity-weighted metallicity). Esto podría introducir algunas diferencias sistemáticas entre los resultados encontrados y las observaciones. Sin embargo, dado que estamos interesadas en las tendencias de la evolución de la MZR, usamos las observaciones como guía y no intentamos corregir cálculos para imitar las observaciones.

\subsection{La relación masa-metalicidad a $z=0$}

La Figura 3.1 muestra la MZR de galaxias con formación de estrellas (galaxias SF) a $z=0$ predicha por la versión de nuestro modelo semi-analítico $\mathrm{SAG}_{\beta 1.9}$ (área sombreada). Definimos como galaxias SF a aquéllas cuya tasa de formación estelar específica sSFR, definida como el cociente entre la SFR y la masa estelar (Capítulo 1), es mayor que $10^{-10.7} \mathrm{yr}^{-1}$, siguiendo el criterio adoptado por Brown et al. (2017) para el análisis de las galaxias satélites seleccionadas por el relevamiento SDSS DR7.

Los valores promedio de la metalicidad para diferentes rangos de masa estelar y su correspondiente desviación estándar se muestran con línea sólida gruesa y barras de error, respectivamente. 
Comparamos la predicción del modelo con los valores promedio de las observaciones espectroscópicas de Tremonti et al. (2004) y Andrews \& Martini (2013), y el ajuste realizado por Maiolino et al. (2008). Este último es calibrado con observaciones espectroscópicas de galaxias a $z=0.07$ de Savaglio et al. (2005), galaxias a $z=2.2$ de Erb et al. (2006), y sus propias observaciones espectroscópicas de galaxias a $z=3.5$. Dicho ajuste está dado por

$$
12+\log (\mathrm{O} / \mathrm{H})=-0.0864\left(\log \mathrm{M}_{\star}-\log \mathrm{M}_{0}\right)^{2}+\mathrm{K}_{0},
$$

donde $\log \mathrm{M}_{0}$ y $\mathrm{K}_{0}$ son estimados a cada redshift, como se muestra en su tabla 5. En general, las masas estelares en los rangos observados se estiman suponiendo una IMF que difiere de la IMF de Chabrier adoptada por el modelo SAG. Tanto Tremonti et al. (2004) y Andrews \& Martini (2013) adoptaron una IMF de Kroupa (Kroupa, 2001). Para realizar, entonces, una comparación justa con SAG, desplazamos sus masas estelares por -0.039 dex (Knebe et al., 2018).

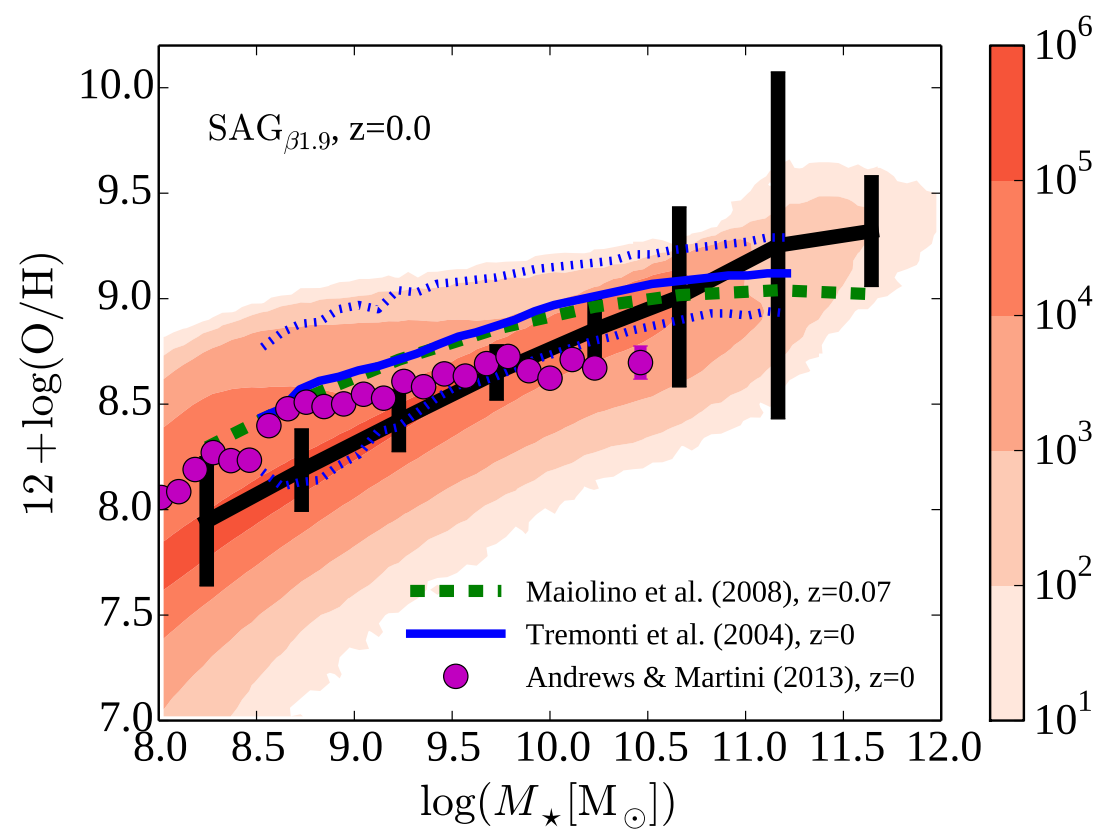

Figura 3.1: MZR de galaxias SF para el modelo $\mathrm{SAG}_{\beta 1.9}$ a $z=0$. La línea negra sólida gruesa representa los valores promedio de la relación, siendo su dispersión de $1 \sigma$ denotada por barras de error. Las regiones sombreadas muestran el histograma 2D de las galaxias modeladas. También se muestra el mejor ajuste de las observaciones de Maiolino et al. (2008, líneas verdes a trazos), los valores promedios y la dispersión de $1 \sigma$ de las observaciones de Tremonti et al. (2004, líneas azules sólidas y punteadas, respectivamente), y los valores promedio de Andrews \& Martini (2013, círculos llenos violetas).

También es interesante enfatizar los diferentes métodos y calibraciones que son usados por estos autores para estimar la metalicidad del gas frío. Tremonti et al. (2004) usaron un modelo teórico de fotoionización que utiliza las líneas de emisión prominentes para estimar una metalicidad estadística. Maiolino et al. (2008) también usaron modelos de fotoionización, aunque sólo para metalicidades de $12+\log (\mathrm{O} / \mathrm{H})>8.35$. Para metalicidades menores, ellos utilizaron un método en donde las mediciones se basan en la temperatura electrónica del gas. Por otro lado, Andrews \& Martini (2013) usaron un método directo (también basado en la temperatura electrónica) para toda su muestra. Como encontraron Kewley \& Ellison (2008), la diferencia entre las estimaciones de la MZR obtenidas por diferentes métodos puede ser hasta de 0.7 dex y puede afectar tanto la forma como la normalización 
de la MZR. En esta línea, Hughes et al. (2013) enfatizaron la importancia de usar el mismo método para obtener metalicidades con el fin de evitar las discrepancias mencionadas.

Sabiendo que las estimaciones de metalicidad no son tarea sencilla, analizamos las predicciones de nuestro modelo para la MZR a $z=0$. SAG $\mathrm{S}_{\beta 1.9}$ produce una MZR con una pendiente más empinada que las observaciones. A $M_{\star} \gtrsim 10^{10} \mathrm{M}_{\odot}$, la MZR modelada está en buen acuerdo con las observaciones dentro de los errores. A $M_{\star} \lesssim 10^{10} \mathrm{M}_{\odot}, \mathrm{SAG}_{\beta 1.9}$ muestra una ligera subestimación de las metalicidades medias, aunque aún se encuentra dentro de las incertezas. En general, la MZR modelada presenta un buen acuerdo con las observaciones a $z=0$.

\subsection{Evolución de la Relación Masa-Metalicidad}

La MZR de galaxias SF a diferentes épocas puede verse en la Figura 3.2 para el modelo $\mathrm{SAG}_{\beta 1.9}$ (área sombreada). La línea sólida gruesa denota los valores de la metalicidad promedio para cada redshift, mientras que la línea sólida fina describe los valores promedio de las galaxias de $\mathrm{SAG}_{\beta 1.9}$ a $z=0$, a modo de comparación. En ambos casos, las barras de error corresponden a la dispersión de $1 \sigma$. Comparamos la MZR modelada con los ajustes observacionales de Maiolino et al. (2008) a $z<3$ y de Troncoso et al. (2014) a $z \sim 3$. Troncoso et al. (2014) toman el ajuste introducido por Maiolino et al. (2008, ecuación 3.1), modificando los parámetros $\log \mathrm{M}_{0}$ y $\mathrm{K}_{0}$ usando sus observaciones espectroscópicas a $3<z<5$ (estas metalicidades fueron obtenidas a través del método empírico $\mathrm{R}_{23}{ }^{1}$ ). A pesar de las diferencias en la forma y normalización de la MZR a $z=0$, las observaciones evidencian una evolución de esta relación.

$\mathrm{A} z=0.7$ (panel superior), podemos ver que hay una tendencia de la MZR modelada a evolucionar hacia metalicidades más bajas a masa estelar fija, comparada con la MZR a $z=0 . \mathrm{SAG}_{\beta 1.9}$ reproduce una relación con una pendiente más empinada (de $\sim 0.40)$ que la observada, aunque se encuentra aún dentro de las incertezas. Por el otro lado, la MZR modelada a $z=2.2$ (panel intermedio) tiene una pendiente de $\sim 0.41$ para todo el rango de masa estelar mostrado, en buen acuerdo con las observaciones. A $z=3.5$ (panel inferior), la MZR modelada está en buen acuerdo con las observaciones a $M_{\star} \lesssim 10^{10} \mathrm{M}_{\odot}$; la pendiente de la relación tanto observada como modelada son similares $(\sim 0.41)$. Sin embargo, para $M_{\star} \gtrsim 10^{10} \mathrm{M}_{\odot}$ la MZR modelada se desvía de la observada, caracterizada por una pendiente más plana.

A pesar de la tensión entre el modelo y las observaciones a $z<1, \mathrm{SAG}_{\beta 1.9}$ logra reproducir la evolución de la normalización de la MZR hasta $z \lesssim 3.5$ (notar que las pendientes de los cuatro redshift estudiados aquí están restringidas a $\approx 0.40-0.41$ ). La evolución con el redshift de la MZR en SAMs ha sido reportado por Xie et al. (2017), aunque para un rango de redshift más acotado. Ellos usan una versión mejorada del SAM GAEA (Hirschmann et al., 2016), el cual fue modificado para agregar una prescripción para la fracción de gas molecular y la eficiencia de formación estelar. Estas mejoras permitieron encontrar una evolución cósmica de la MZR hasta $z \sim 0.7$ para $10^{8} \lesssim M_{\star} / \mathrm{M}_{\odot} \lesssim 10^{12} \mathrm{y}$ hasta $z \sim 2$ para $M_{\star} \gtrsim 10^{10} \mathrm{M}_{\odot}$. En su lugar, $\mathrm{SAG}_{\beta 1.9}$ produce una evolución de la MZR hasta $z \sim 3.5$ y $z \sim 2.2$ para $M_{\star} \lesssim 10^{10.5} \mathrm{M}_{\odot}$ y $M_{\star} \gtrsim 10^{10.5} \mathrm{M}_{\odot}$, respectivamente. En las siguientes secciones estudiamos cómo el cambio en el modelado de feedback de SN y el destino del material reciclado pueden alterar el contenido de masa y metales de las diferentes componente bariónicas. Debido a que estos cambios quedan plasmados en la MZR, este estudio resulta importante para contribuir a nuestro entendimiento sobre el origen de su evolución.

\footnotetext{
${ }^{1} \mathrm{El}$ método $\mathrm{R}_{23}$ utiliza un parámetro empírico que permite obtener la abundancia química del gas en función del cociente entre las líneas intensas de emisión del oxígeno y el hidrógeno (generalmente el cociente ([O II] $\lambda 3727+$ [O III] $\lambda \lambda 4959$; 5007) / $\mathrm{H}_{\beta}$; Pagel et al. 1979). Además, este método utiliza la temperatura electrónica de las líneas para su calibración.
} 

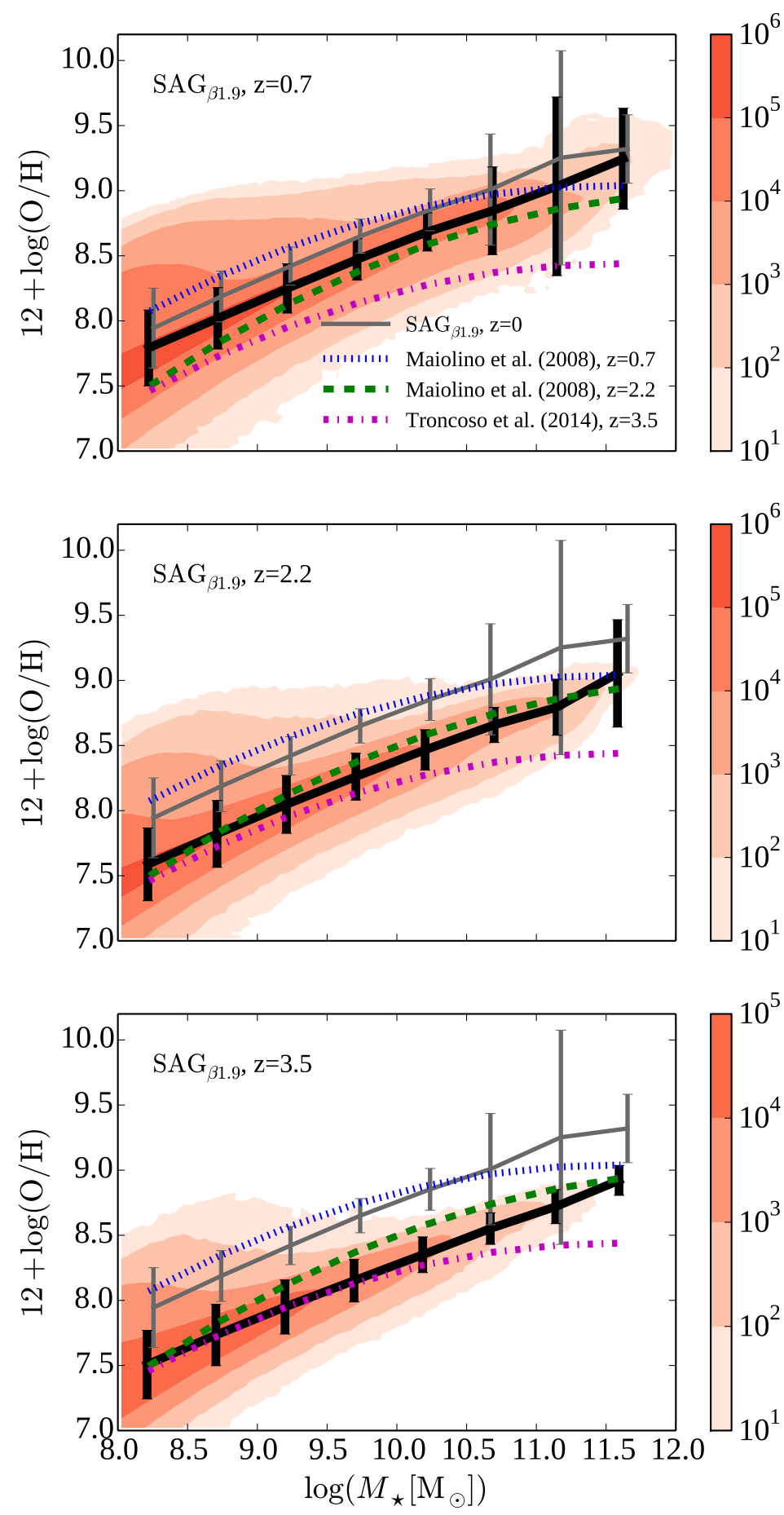

Figura 3.2: MZR de galaxias SF generadas por $\mathrm{SAG}_{\beta 1.9}$ a $z=0.7$ (panel superior), $z=2.2$ (panel intermedio) y $z=3.5$ (panel inferior). Las regiones sombreadas muestran el histograma 2D de las galaxias modeladas, mientras que la línea negra sólida gruesa representa los valores promedio de la relación con su correspondiente dispersión de $1 \sigma$ mostrada con barras de error. Como referencia, cada panel muestra la relación a $z=0$ generada por $\mathrm{SAG}_{\beta 1.9}$ con línea sólida fina. En todos los casos, los ajustes de la MZR de Maiolino et al. (2008) a $z=0.7$ y $z=2.2$, y los de Troncoso et al. (2014) a $z=3.5$ son mostrados con líneas azul punteada, verde a trazos y violeta a trazos punteada, respectivamente. 


\subsection{El origen de la Evolución de la MZR}

Este capítulo tiene como fin identificar los procesos físicos claves que resulten responsables de la evolución de la MZR. En C18 se muestra que el modelado del feedback de SN tiene un gran impacto en la evolución de la SFR cósmica y el ensamble de masas de galaxias, siendo fuertemente dependiente del valor que toma el parámetro que regula la dependencia de la eficiencia del feedback de $\mathrm{SN}$ con el redshift, $\beta$ (Ecuación 2.10). Siendo éste un mecanismo que puede tener un rol importante en la evolución de la MZR, estudiamos propiedades relacionadas de la población de galaxias generadas por los modelos $\mathrm{SAG}_{\beta 1.9}, \mathrm{SAG}_{\beta 1.3}$ y $\mathrm{SAG}_{\beta 0.0}$. A estos, agregamos el análisis de la población de galaxias generadas por el modelo $\mathrm{SAG}_{\beta 1.9-\mathrm{Rec}}$, el cual nos permite evaluar el impacto del destino del material reciclado. Las características de estos modelos fueron descritas en la Sección 2.1.6.

\subsubsection{Comparación de la MZR predicha por variantes de SAG}

La MZR de galaxias $\mathrm{SF}$ a diferentes redshifts predicha por los modelos $\mathrm{SAG}_{\beta 1.9}, \mathrm{SAG}_{\beta 1.3}, \mathrm{SAG}_{\beta 0.0}$ y $\mathrm{SAG}_{\beta 1.9-\mathrm{Rec}}$ es presentada en los paneles superior izquierdo, superior derecho, inferior izquierdo e inferior derecho de la Figura 3.3, respectivamente. Las líneas muestran los valores promedios de la relación a $z=0$ (línea sólida gruesa negra), $z=0.7$ (línea a trazos gruesa roja), $z=2.2$ (línea a trazos fina azul) y $z=3.5$ (línea sólida fina verde). Tanto el modelo $\mathrm{SAG}_{\beta 1.9}$ como el $\mathrm{SAG}_{\beta 1.3}$ muestran una clara evolución de la MZR que no se ve en $\mathrm{SAG}_{\beta 0.0}$. Las modificaciones introducidas al modelado de feedback de SN, descritas en la Sección 2.1.3, llevan a la evolución de la MZR, resultado evidente al observar el decrecimiento de su normalización al incrementar el redshift. A este resultado se suma que la evolución de la MZR producto del modelo $\mathrm{SAG}_{\beta 1.9}$ es más pronunciada que para $\mathrm{SAG}_{\beta 1.3}$. Considerando el rango de redshift $0 \leq z \leq 3.5$, la evolución obtenida por la normalización de la MZR es de $\sim 0.44$ dex para $\mathrm{SAG}_{\beta 1.9} \mathrm{y} \sim 0.28$ dex para $\mathrm{SAG}_{\beta 1.3}$. Esta diferencia en la evolución es una consecuencia de una dependencia con el redshift más suave en las masas recalentadas y eyectadas de $\mathrm{SAG}_{\beta 1.3}$ en relación a $\mathrm{SAG}_{\beta 1.9}(\beta=1.3$ contra $\beta=1.99)$.

Por otro lado, la evolución de la MZR es $\sim 0.35$ dex para el modelo $\mathrm{SAG}_{\beta 1.9-\mathrm{Rec}}$, un poco menos que para $\mathrm{SAG}_{\beta 1.9}$, aunque las características del modelado de $\mathrm{SN}$ son las mismas en ambos modelos. Esto implica que, a pesar de que el feedback de SN pareciera ser el principal conductor de la evolución de la MZR, el cambio en el destino del material reciclado también tiene impacto, aunque leve, en el grado de la misma. La forma en la que el material reciclado afecta las fases de gas frío y caliente en el modelo $\mathrm{SAG}_{\beta 1.9}$ permite que éste sea capaz de reproducir la evolución observada de la MZR (ver Figura 3.2). La razón de esto es analizada con más detalle en la Sección 3.3.3.

La pendiente de la MZR ${ }^{2}$ no muestra un cambio significativo al pasar el tiempo en ninguno de los modelos. Para el rango de redshift considerado aquí, las pendientes se encuentran comprendidas entre los valores $\sim 0.42-0.44, \sim 0.44-0.45, \sim 0.39-0.38, \mathrm{y} \sim 0.39-0.40$ para $\mathrm{SAG}_{\beta 1.9}, \mathrm{SAG}_{\beta 1.3}$, $\mathrm{SAG}_{\beta 0.0}$ y $\mathrm{SAG}_{\beta 1.9-\mathrm{Rec}}$, respectivamente. Una predicción similar es obtenida por Ma et al. (2016) de su análisis de las simulaciones FIRE, quienes encontraron que una pendiente común de $\sim 0.35$ para todos los redshifts del rango $z \in[0,6]$ provee un buen ajuste para sus resultados modelados. Notar que la normalización de su MZR evoluciona por $\sim 1$ dex dentro de ese rango de redshift, la cual es del orden de la hallada por $\mathrm{SAG}_{\beta 1.9}$ cuando se restringe al rango de redshift que analizamos aquí. Guo et al. (2016) compararon las predicciones de la simulación hidrodinámica EAGLE de referencia y dos modelos semi-analíticos, GALFORM y L-GALAXIES, y encontraron que mientras el primero muestra una fuerte evolución de la MZR tanto en pendiente como en normalización, los SAMs tienen una MZR que apenas evoluciona con el redshift. De esta manera, logramos obtener resultados que no sólo son comparables con las observaciones, sino también con los resultados de simulaciones cosmológicas.

\footnotetext{
${ }^{2}$ La pendiente de la MZR es estimada al aplicar un ajuste de mínimos cuadrados a los rangos de masa estelar $M_{\star} / \mathrm{M}_{\odot} \in$ $\left[10^{8}, 10^{10.5}\right]$, donde la dispersión de $1 \sigma$ es baja, como se muestra en la Figura 3.3.
} 

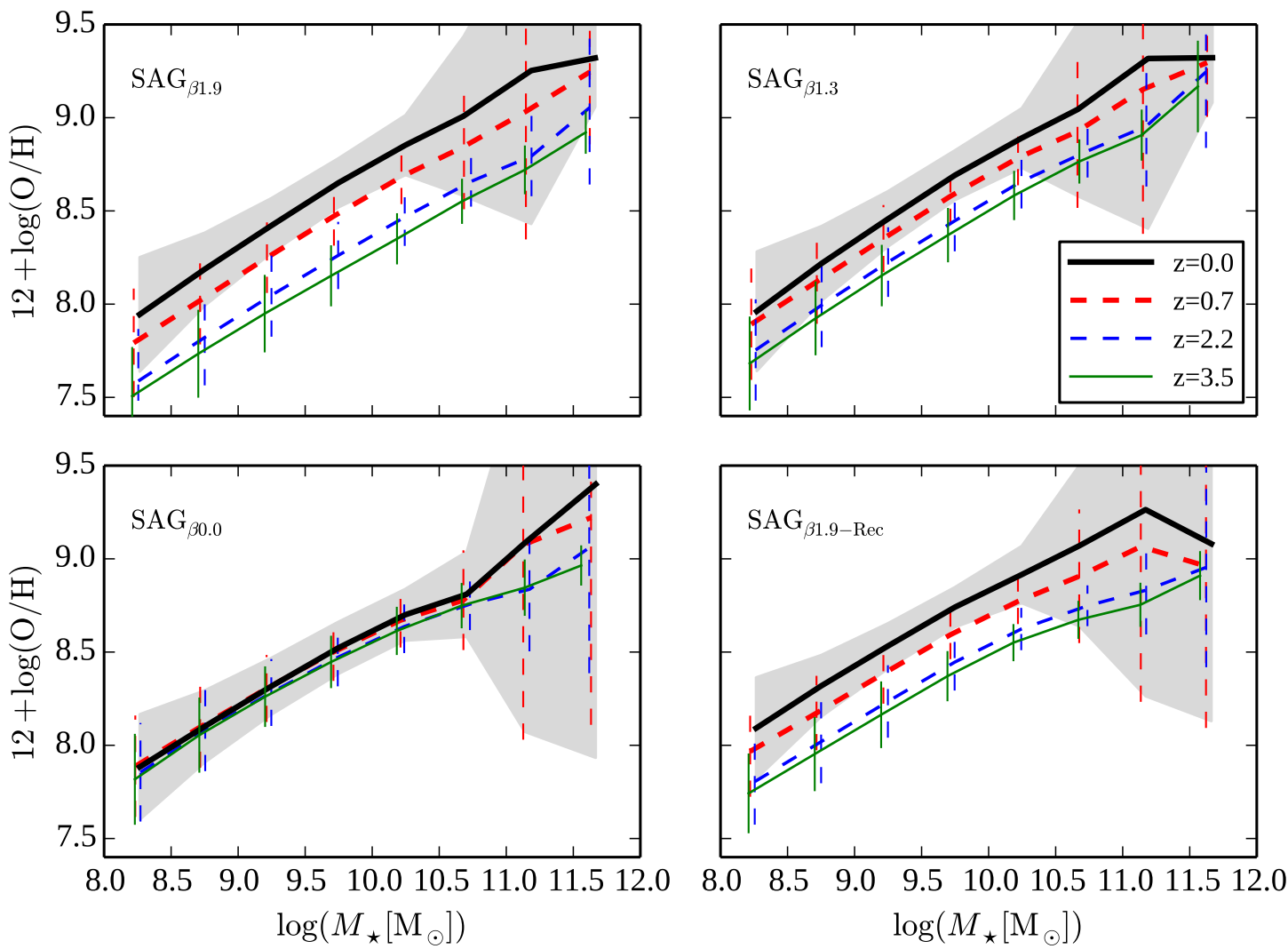

Figura 3.3: MZR de galaxias SF a diferentes redshifts para los modelos $\mathrm{SAG}_{\beta 1.9}$ (panel superior izquierdo), $\mathrm{SAG}_{\beta 1.3}$ (panel superior derecho), $\mathrm{SAG}_{\beta 0.0}$ (panel inferior izquierdo) y $\mathrm{SAG}_{\beta 1.9-\mathrm{Rec}}$ (panel inferior derecho). Los diferentes tipos de líneas denotan los valores promedio de la MZR a $z=0,0.7,2.2$ y 3.5, como indica la leyenda. La dispersión de $1 \sigma$ se muestra como áreas sombreadas para $z=0$ y con barras de error para mayores redshifts. Los modelos con la nueva receta de feedback de SN muestran algún nivel de evolución en su MZR, contrario al modelo $\mathrm{SAG}_{\beta 0.0}$ que no muestra evolución alguna. La evolución de la MZR del modelo $\mathrm{SAG}_{\beta 1.9}$ es del orden de lo que se encuentra observacionalmente ( $\approx 0.5$ dex, ver Figura 3.2).

\subsubsection{Evolución de la densidad cósmica del gas frío y la SFR}

La evolución del contenido de gas y metales del ISM lleva a la evolución de la MZR. Por lo tanto, nos enfocamos en la densidad cósmica del gas frío, $\Omega_{\text {cold }}$, como función del redshift, prestando especial atención a la densidad cósmica de las componentes de hidrógeno y oxígeno de esta fase $\left(\Omega_{\mathrm{H}}\right.$ y $\Omega_{\mathrm{O}}$, respectivamente). La densidad del gas es calculada como

$$
\Omega_{\mathrm{x}}=\frac{\rho_{\mathrm{x}}}{\rho_{\mathrm{c}(\mathrm{z}=0)}}=\frac{M_{\mathrm{x}} / L^{3}}{\rho_{\mathrm{c}(\mathrm{z}=0)}},
$$

donde $\rho_{\mathrm{c}(\mathrm{z}=0)}=2.77 \times 10^{11} h^{2} \mathrm{M}_{\odot} \mathrm{Mpc}^{-3}$ es la densidad crítica a $z=0$ para la cosmología adoptada, y $\rho_{\mathrm{x}}$ es la densidad de masa del gas frío total $(\mathrm{x}=$ cold $)$, o del hidrógeno $(\mathrm{x}=\mathrm{H})$ y oxígeno $(\mathrm{x}=\mathrm{O})$ contenidos en el gas frío. La densidad de masa $\rho_{\mathrm{x}}$ es estimada por el cociente entre la correspondiente masa total, $M_{\mathrm{x}}$, en la simulación y el cubo del lado de la caja cosmológica, $L$, de la simulación comológica subyacente.

La Figura 3.4 muestra, para el modelo $\mathrm{SAG}_{\beta 1.9}$, la densidad cósmica $\Omega_{\text {cold }}$ (línea sólida), $\Omega_{\mathrm{H}}$ (línea punteada) y $\Omega_{\mathrm{O}}$ (línea gruesa a trazos) como función del redshift. Comparamos estos resultados con el compilado de observaciones de Lagos et al. (2014), quienes estudian la densidad del gas de hidrógeno 
atómico neutro (HI) y molecular $\left(\mathrm{H}_{2}\right)$. Dado que el gas frío en el modelo no está dividido en estas dos componentes, la comparación se realiza con la densidad de gas observada que resulta de la suma de $\mathrm{HI}$ y $\mathrm{H}_{2}$. Para el rango de redshift cosiderado aquí, $\Omega_{\mathrm{H}}$ concuerda con la densidad cósmica observada del hidrógeno, $\mathrm{HI}+\mathrm{H}_{2}$, razonablemente bien. Este acuerdo no es necesariamente sorprendente dado que el modelo $\mathrm{SAG}_{\beta 1.9}$ ha sido calibrado para coincidir con la fracción de gas frío a $z=0$, entre otras restricciones (ver Sección 2.1). El acuerdo de $\Omega_{\mathrm{H}}$ con la observada $\mathrm{HI}+\mathrm{H}_{2}$ es particularmente bueno a $z \approx 1$. Para redshifts menores (mayores), la predicción de la densidad cósmica del hidrógeno es subestimada (sobreestimada). Esto es consistente con las discrepancias entre las predicciones del modelo y las observaciones encontradas en la evolución de la SFRD para el modelo $\mathrm{SAG}_{\beta 1.9}$, como veremos a continuación.

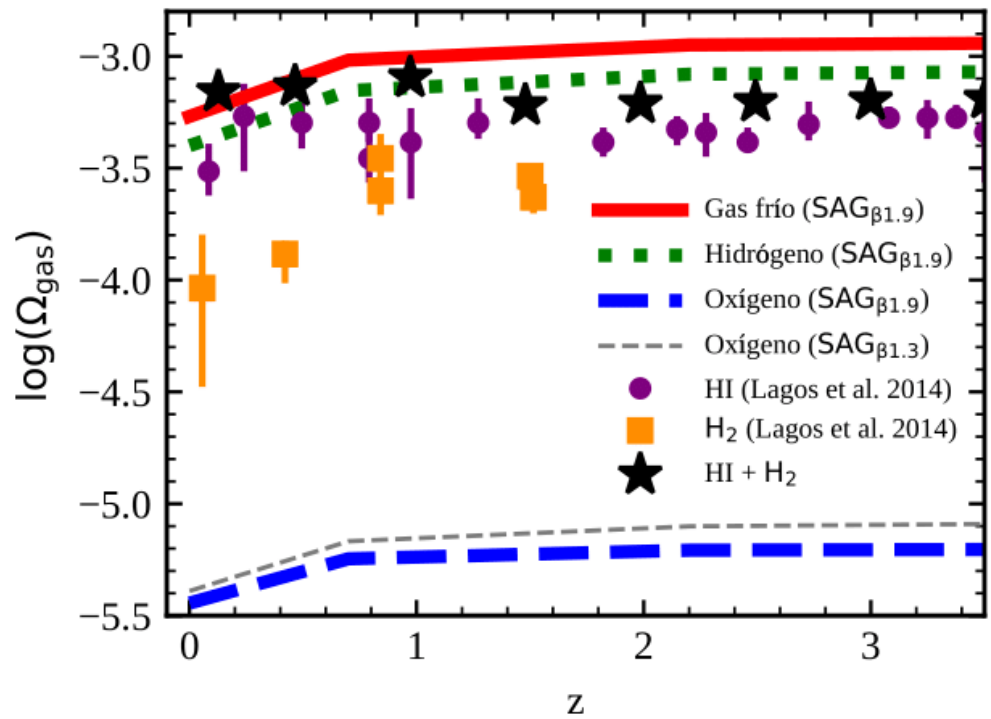

Figura 3.4: Evolución de la densidad cósmica del gas frío $\left(\Omega_{\text {cold }}\right.$, línea roja sólida), del hidrógeno contenido en esta fase $\left(\Omega_{\mathrm{H}}\right.$, línea verde punteada) y de la componente de oxígeno ( $\Omega_{\mathrm{O}}$, línea azul gruesa a trazos) para el modelo $\mathrm{SAG}_{\beta 1.9}$. La densidad cósmica del contenido de oxígeno en el gas frío para el modelo $\mathrm{SAG}_{\beta 1.3}$ es representada con una línea gris a trazos fina. Estrellas negras muestran la densidad de gas observada obtenida de la suma de las masas de hidrógeno atómico neutro (HI, círculos violetas) e hidrógeno molecular ( $\mathrm{H}_{2}$, cuadrados naranjas) presentados por Lagos et al. (2014). La densidad cósmica modelada del hidrógeno muestra un buen acuerdo con las observaciones en todo el rango de redshift considerado $(0 \leq z \leq 3.5)$.

La Figura 3.5 presenta la evolución de la SFRD cósmica para los modelos $\mathrm{SAG}_{\beta 1.9}, \mathrm{SAG}_{\beta 1.3}$ y $\mathrm{SAG}_{\beta 0.0}$. A su vez, estos resultados son comparados con los datos compilados de Behroozi et al. (2013a) y los últimos resultados combinados de GAMA/COSMOS/3DHST de Driver et al. (2018). Los tres modelos muestran acuerdos razonables con las observaciones, en especial a $z<2$, sin embargo existen algunas discrepancias que merecen discutirse. El modelo $\mathrm{SAG}_{\beta 1.9}$ muestra una subestimación de la SFRD a $z \gtrsim 2$, y una sobreestimación a $z \lesssim 0.5$. Esta diferencia explica la desviación de $\Omega_{\mathrm{H}}$ respecto de los valores observacionales mencionada previamente (Figura 3.4), debido a que la formación estelar se produce a partir del reservorio de gas frío.

$\mathrm{El}$ acuerdo entre las predicciones del modelo y las observaciones mejora, particularmente a alto redshift, para el modelo $\mathrm{SAG}_{\beta 1.3}$. Esto es esperable dado que el parámetro $\beta$, encargado de regular la dependencia con el redshift de la masa recalentada y eyectada (Ecuaciones 2.10 y 2.12, respectivamente), se fija a un valor más pequeño en $\mathrm{SAG}_{\beta 1.3}$ comparado con $\mathrm{SAG}_{\beta 1.9}$. Entonces, el efecto más débil del feedback de $\mathrm{SN}$ a redshifts mayores en el modelo $\mathrm{SAG}_{\beta 1.3}$ da lugar a una mayor cantidad de gas frío disponible para la formación estelar, traduciéndose en una SFRD mayor en comparación con 
$\mathrm{SAG}_{\beta 1.9}$. Este efecto se exacerba en el modelo $\mathrm{SAG}_{\beta 0.0}$, donde el gas recalentado es igual de eficiente, a SFR y velocidad circular fijas, para todo redshift, en contraste con la eficiencia que evoluciona en $\mathrm{SAG}_{\beta 1.3}$ y $\mathrm{SAG}_{\beta 1.9}$, permitiendo una formación estelar significantemente mayor a alto redshift.

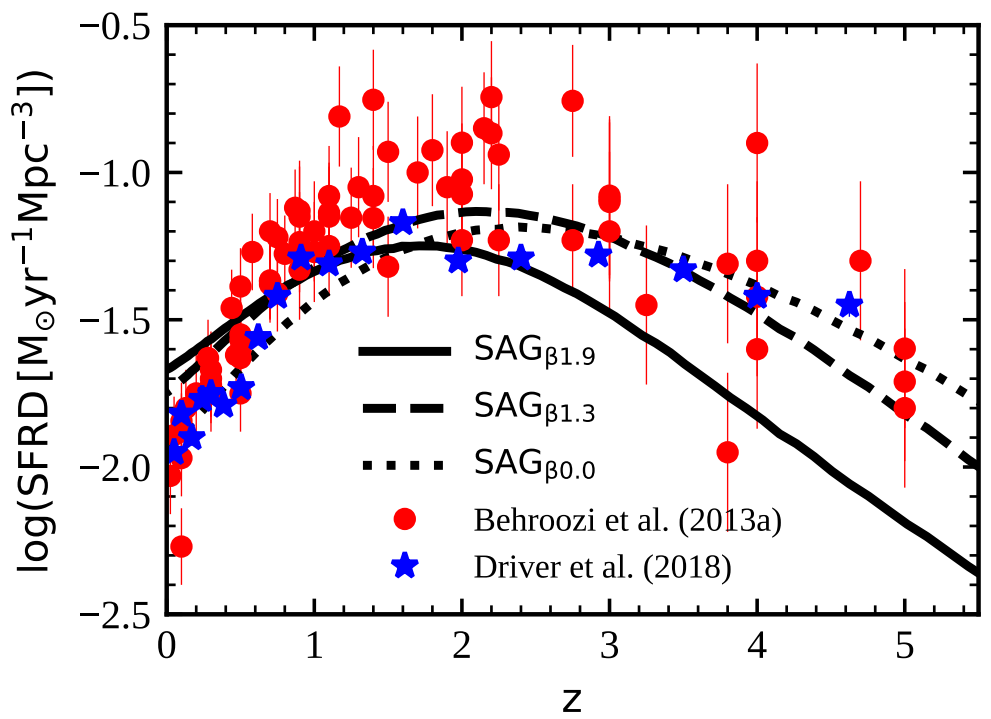

Figura 3.5: Densidad cósmica de SFR como función del redshift para los modelos $\mathrm{SAG}_{\beta 1.9}$ (línea sólida), $\mathrm{SAG}_{\beta 1.3}$ (línea a trazos) y $\mathrm{SAG}_{\beta 0.0}$ (línea punteada). Los círculos con barras de error muestran las observaciones compiladas por Behroozi et al. (2013a), mientras que las estrellas corresponden a resultados recientes de Driver et al. (2018). En general, los tres modelos muestran acuerdo con las observaciones. Las diferencias aparecen por los diversos modelados del feedback de $\mathrm{SN}$ (entre $\mathrm{SAG}_{\beta 1.9}$ y $\mathrm{SAG}_{\beta 0.0}$ ) o por los distintos valores del parámetro $\beta$ asociado al nuevo modelo de feedback de $\mathrm{SN}$ (entre $\mathrm{SAG}_{\beta 1.9}$ y $\mathrm{SAG}_{\beta 1.3}$ ).

La Figura 3.6 muestra los valores promedios de la masa recalentada como función de la masa estelar de las galaxias centrales a redshift $z=0$ (panel superior izquierdo), $z=0.7$ (panel superior derecho), $=2.2$ (panel inferior izquierdo) y $z=3.5$ (panel inferior derecho). Las líneas sólida y a trazos muestran los modelos $\mathrm{SAG}_{\beta 1.9}$ y $\mathrm{SAG}_{\beta 0.0}$, respectivamente; las regiones sombreadas muestran la correspondiente dispersión de $1 \sigma$. Nos concentramos en los modelos $\mathrm{SAG}_{\beta 1.9}$ y $\mathrm{SAG}_{\beta 0.0}$ porque éstos producen la mayor y menor evolución de la normalización de la MZR, respectivamente. Podemos ver que la cantidad de metales en la masa recalentada, a masa estelar fija, muestra una leve dependencia con el redshift en el modelo $\mathrm{SAG}_{\beta 0.0}$. Por el contrario, $\mathrm{SAG}_{\beta 1.9}$ muestra que, también a masa estelar fija, la cantidad de masa recalentada decrece fuertemente al decrecer el redshift. Esto es esperable debido a la explícita dependencia con el redshift introducida en la masa recalentada producto del nuevo modelo de feedback de SN (Ecuación 2.10). Esto resulta en mayores cantidades de masa recalentada que en $\mathrm{SAG}_{\beta 0.0}$ a $z \gtrsim 2.2$ y menores cantidades a redshifts menores.

Los valores bajos de SFRD a altos redshifts en el modelo $\mathrm{SAG}_{\beta 1.9}$ implican que la cantidad de metales inyectados por estrellas evolucionadas al ISM es menor que en $\mathrm{SAG}_{\beta 1.3}$ y $\mathrm{SAG}_{\beta 0.0}$. En efecto, la densidad cósmica del oxígeno es menor en $\mathrm{SAG}_{\beta 1.9}$ que en $\mathrm{SAG}_{\beta 1.3}$ a todo redshift; este último es representado por una línea fina a trazos en la Figura 3.4. Además, la tasa del enriquecimiento químico también difiere, como se muestra por las diferentes dependencias de redshifts de $\Omega_{\mathrm{O}}$ en estos modelos. Cuantificamos este resultado al estimar las pendientes de la relación $\Omega_{0}-z$ para los rangos de redshift por encima y debajo de $\approx 0.7$, donde la relación presenta un quiebre. Para $z \gtrsim 0.7$, estas pendientes alcanzan valores de $\approx 0.015 \mathrm{y} \approx 0.028$ para los modelos $\mathrm{SAG}_{\beta 1.9}$ y $\mathrm{SAG}_{\beta 1.3}$, respectivamente. Estos valores crecen para $z \lesssim 0.7$, siendo $\approx 0.28 \mathrm{y} \approx 0.32$, respectivamente. Aplicando el mismo análisis 
para $\Omega_{\mathrm{H}}$, las pendientes son $\approx 0.04$ para $z \gtrsim 0.7 \mathrm{y} \approx 0.35$ para $z \lesssim 0.7$, tanto para $\mathrm{SAG}_{\beta 1.9}$ como para $\mathrm{SAG}_{\beta 1.3}$. Las densidades cósmicas $\Omega_{\text {cold }}$ y $\Omega_{\mathrm{H}}$ son bastante similares en estos dos modelos y, por claridad, sólo mostramos este último para el modelos $\mathrm{SAG}_{\beta 1.33}$. Podemos ver que, a un dado rango de redshift, las diferencias entre las pendientes de las relaciones $\Omega_{\mathrm{O}}-z$ y $\Omega_{\mathrm{H}}-z$ son mayores para $\mathrm{SAG}_{\beta 1.9}$ que para $\mathrm{SAG}_{\beta 1.3}$, lo que indicaría que el gas frío se contamina de manera más gradual cuando la eficiencia de feedback de SN es mayor a mayores redshifts. Este hecho se traduce en una evolución apreciable de la MZR. Este análisis apoya la justificación de la poca evolución de la MZR predicha por los diversos SAMs discutidos por Hirschmann et al. (2016), en donde ésto se le atribuye a la alta SFR y al consecuente rápido enriquecimiento químico de la fase de gas frío a alto redshift, lo que resulta en tasas de recalentamiento y eyección más bien lentas a tiempos tempranos.
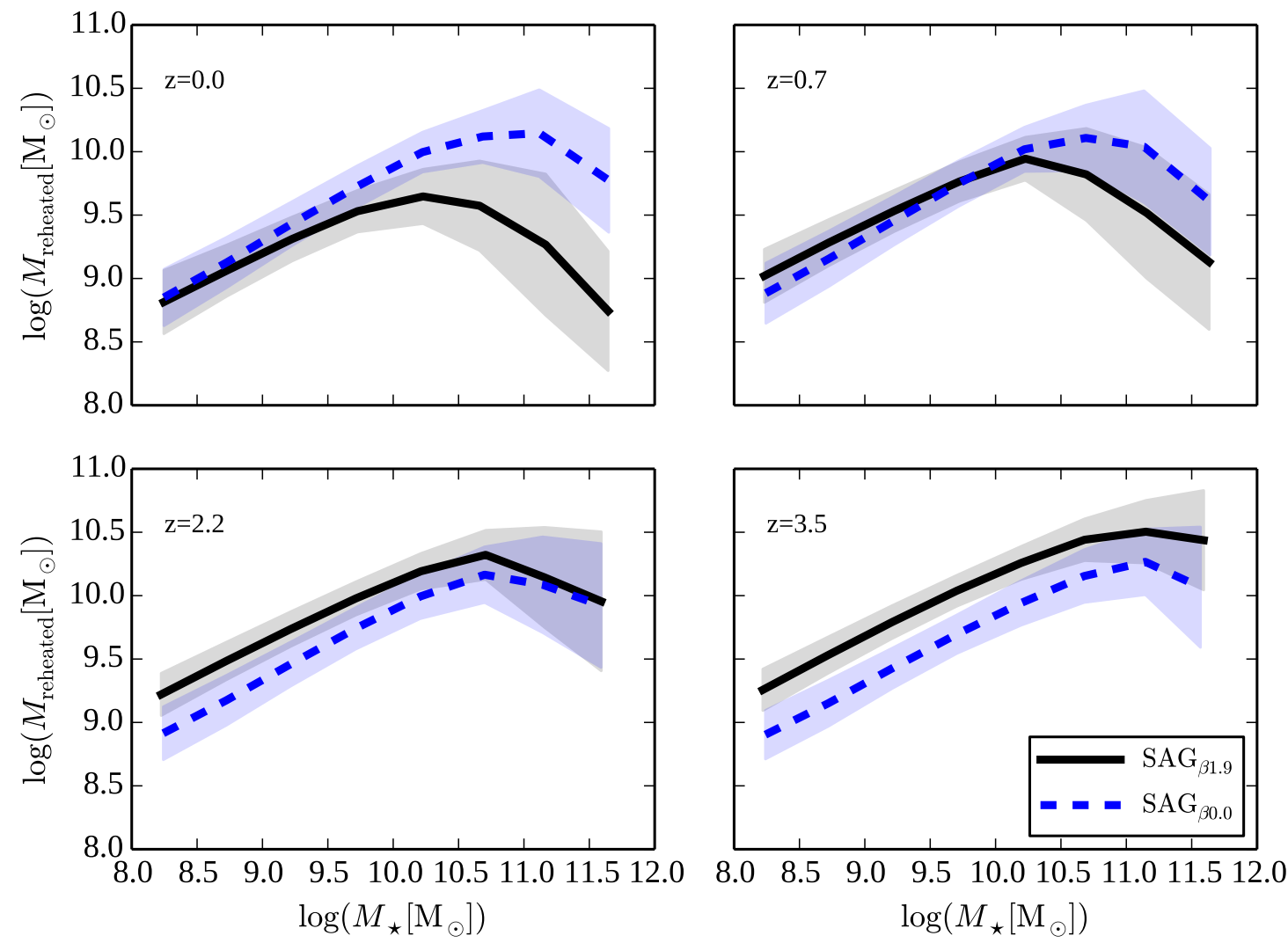

Figura 3.6: Masa recalentada de las galaxias $\mathrm{SF}$ en función de la masa estelar a $z=0$ (panel superior izquierdo), $z=0.7$ (panel superior derecho), $z=2.2$ (panel inferior izquierdo) y $z=3.5$ (panel inferior derecho). Las líneas negras sólidas y azules a trazos representan los modelos $\mathrm{SAG}_{\beta 1.9}$ y $\mathrm{SAG}_{\beta 0.0}$, respectivamente, mientras que las regiones sombreadas negras y azules muestran su correspondiente dispersión de $1 \sigma$.

Así, el decrecimiento del grado de evolución de la normalización de la MZR está directamente relacionado con el progresivo incremento de la SFRD a $z \gtrsim 2$, a medida que se reduce la fuerza de la dependencia con el redshift de la masa recalentada de $\mathrm{SAG}_{\beta 1.9}$ a $\mathrm{SAG}_{\beta 0.0}$. El feedback de $\mathrm{SN}$, entonces, afecta la evolución de la MZR a través del rol que tiene en la regulación de la formación estelar, que a su vez determina la cantidad de gas recalentado (Ecuaciones 2.9 y 2.10) y la masa reciclada que contiene los metales recientemente sintetizados (Ecuaciones 2.14 y 2.17). Debido a que la metalicidad del gas frío está dada por la proporción relativa de metales y masa que vuelve al ISM, es el balance entre la caída y eyección de material lo que transporta masa y metales entre diferentes componentes bariónicas (Ecuaciones 2.18, 2.19 y 2.20), determinando el grado de evolución de la 
MZR.

\subsubsection{El rol de la dilución de metales en eyecciones de gas}

Hasta ahora, hemos mostrado que el gas frío se contamina más gradualmente en el tiempo cuando el efecto del feedback de SN es grande a alto redshift, lo que da lugar a una mayor evolución de la normalización de la MZR. Las explosiones de SNs no sólo inyectan energía al ISM y al medio intergaláctico (IGM, por Intergalactic Medium), sino que además inyectan metales recientemente sintetizados que son contenidos en la masa reciclada. También hemos visto que el destino de la masa reciclada juega un rol en la determinación de la magnitud de la evolución de la MZR (Sección 3.3.1). De acuerdo con los dos posibles esquemas de regulación de transferencia de masa entre las componentes bariónicas adoptadas (Sección 2.1.4), el destino de la masa reciclada puede depender tanto de su relativa proporción con respecto a la masa recalentada (como en los modelos $\mathrm{SAG}_{\beta 1.9}, \mathrm{SAG}_{\beta 1.3}$ y $\mathrm{SAG}_{\beta 0.0}$ ) o en la fracción de gas frío (como en el modelo $\mathrm{SAG}_{\beta 1.9-\mathrm{Rec}}$ ). Nos referimos al primero como el esquema de reciclado $\mathcal{A}$ y al segundo como al esquema de reciclado $\mathcal{B}$. Analizamos, entonces, el camino en los que estos esquemas tienen injerencia en la metalicidad del gas frío. De aquí en más, mostraremos resultados de los modelos $\mathrm{SAG}_{\beta 1.9}, \mathrm{SAG}_{\beta 0.0}$ y $\mathrm{SAG}_{\beta 1.9-\mathrm{Rec}}$ debido a las diferencias significativas en sus predicciones.

La Figura 3.7 muestra la fracción de masa reciclada que es transferida directamente al gas frío, $M_{\text {recycled }}^{\text {cold }}$, comparada con la masa total reciclada, $M_{\text {recycled }}$ (es decir, $f_{\text {recycled }}^{\text {cold }}=M_{\text {recycled }}^{\text {cold }} / M_{\text {recycled }}$ ), como función de la masa estelar de galaxias $\mathrm{SF}$ a $z=0$ (panel superior izquierdo), $z=0.7$ (panel superior derecho), $z=2.2$ (panel inferior izquierdo) y $z=3.5$ (panel inferior derecho). Los modelos $\mathrm{SAG}_{\beta 1.9}, \mathrm{SAG}_{\beta 0.0}$ y $\mathrm{SAG}_{\beta 1.9-\operatorname{Rec}}$ son representados con líneas sólida, a trazos, y punteada, respectivamente. Los diferentes esquemas adoptados que definen el destino de la masa reciclada producen tendencias completamente opuestas. La fracción de masa reciclada que es depositada en la fase de gas frío incrementa con la masa estelar en el esquema de reciclado $\mathcal{A}$, mientras que decrece para galaxias más masivas en el esquema de reciclado $\mathcal{B}$.

Los modelos $\mathrm{SAG}_{\beta 1.9}$ y $\mathrm{SAG}_{\beta 0.0}$, que adoptan el esquema de reciclado $\mathcal{A}$, tienen $f_{\text {recycled }}^{\text {cold }} \approx 0$ para galaxias de baja masa $\left(M_{\star} \lesssim 10^{10} \mathrm{M}_{\odot}\right)$ debido a que la masa recalentada es mayor que la masa reciclada para estas galaxias en el modelo. Esto implica que los metales recientemente sintetizados son inyectados directamente en el halo de gas caliente. Para galaxias con $M_{\star} \gtrsim 10^{10} \mathrm{M}_{\odot}$ a un dado redshift, la fracción $f_{\text {recycled }}^{\text {cold }}$ incrementa con la masa estelar para ambos modelos, consistente con el hecho de que la masa recalentada decrece, como puede verse en la Figura 3.6, permitiendo que más masa reciclada sea transferida directamente al gas frío. A su vez, en este rango de masa estelar puede verse que, fijando la masa estelar, la dependencia con el redshift de la fracción $f_{\text {recycled }}^{\text {cold }}$ difiere entre los modelos, característica propia de las diferentes recetas de feedback de SN. Mientras $f_{\text {recycled }}^{\text {cold }}$ incrementa progresivamente cuando el redshift decrece para $\mathrm{SAG}_{\beta 1.9}$, lo opuesto sucede para $\mathrm{SAG}_{\beta 0.0}$. La dependencia con el redshift de la eficiencia de feedback de $\mathrm{SN}$ en $\mathrm{SAG}_{\beta 1.9}$ produce menos masa recalentada a bajo redshift (ver Figura 3.6), de modo que el $\approx 80 \%$ de la masa reciclada termina en el gas frío para las galaxias más masivas a $z=0$.

En el esquema de reciclado $\mathcal{B}, f_{\text {recycled }}^{\text {cold }} \gtrsim 0.5$ para galaxias de baja masa a todo redshift. Esta fracción decrece para las galaxias más masivas, especialmente a bajo redshift $(z \lesssim 0.7)$. Estas tendencias son el resultado de la dependencia de la fracción de gas frío con la masa estelar, la cual es mayor para galaxias menos masivas (ver Figura 2.3). Por lo tanto, mientras que galaxias de baja masa tienen gas frío principalmente contaminado por el material reciclado que se deposita en su reservorio, la baja fracción de gas frío en galaxias masivas produce una mayor fracción de material reciclado que será eyectado al halo de gas caliente. Entonces, el gas frío de las galaxias de alta masa es enriquecido químicamente mediante el proceso de enfriamiento del gas. Aunque la tasa de enfriamiento del gas se vuelve menor para galaxias masivas como resultado del feedback de AGN, el flujo de gas contami- 
nado que se enfría tiene un impacto importante en el enriquecimiento químico de la fase fría debido a la baja fracción de gas frío de estas galaxias.
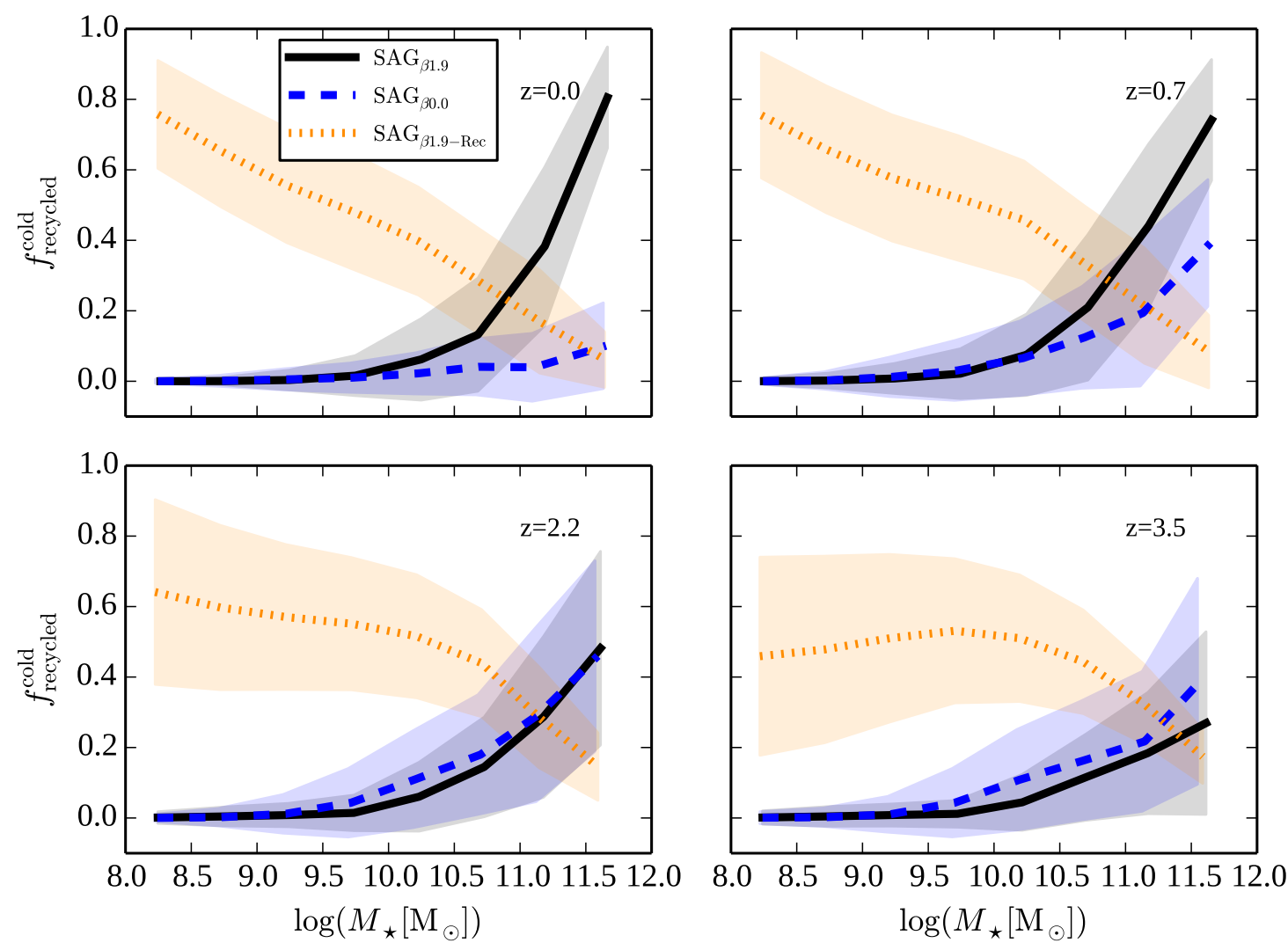

Figura 3.7: Fracción de masa reciclada que es transferida directamente a la fase de gas frío con respecto al total de la masa reciclada $\left(f_{\text {recycled }}^{\text {cold }}=M_{\text {recycled }}^{\text {cold }} / M_{\text {recycled }}\right)$, como función de la masa estelar de galaxias SF a $z=0$ (panel superior izquierdo), $z=0.7$ (panel superior derecho), $z=2.2$ (panel inferior izquierdo) y $z=3.5$ (panel inferior derecho). Líneas negras sólidas, azules a trazos y naranjas punteadas muestran los resultados de los modelos $\mathrm{SAG}_{\beta 1.9}, \mathrm{SAG}_{\beta 0.0}$ y $\mathrm{SAG}_{\beta 1.9-\mathrm{Rec}}$, respectivamente. Los dos primeros modelos usan el esquema de reciclado $\mathcal{A}$, mientras que el tercero usa el esquema de reciclado $\mathcal{B}$. Las regiones sombreadas negra, azul y naranja representan las correspondientes dispersiones de $1 \sigma$.

\subsubsection{La importancia el enriquecimiento químico de la fase caliente del gas}

Por lo visto anteriormente, la contaminación del gas frío se produce mediante dos canales, específicamente, la contaminación directa del gas frío debido al material reciclado que es inyectado directamente a esta fase (casos con valores altos de $f_{\text {recycled }}^{\text {cold }}$ ) y por enfriamiento radiativo del gas, que contribuye con una masa de gas frío con metalicidad determinada por la que consiguió el halo de gas caliente (casos con valores bajos de $f_{\text {recycled }}^{\text {cold }}$. Para una dada masa estelar, la importancia relativa de cada canal depende del esquema de reciclado adoptado. Para los casos donde domina el enfriamiento radiativo del gas, la metalicidad del gas caliente es el motor principal de la metalicidad del gas frío. En esta línea, la Figura 3.8 muestra los valores promedio de la metalicidad del gas caliente, $Z_{\mathrm{hot}}$, como función de la masa estelar de galaxias $\mathrm{SF}$ en los modelos $\mathrm{SAG}_{\beta 1.9}$ (línea sólida), $\mathrm{SAG}_{\beta 0.0}$ (línea a trazos) y $\mathrm{SAG}_{\beta 1.9-\mathrm{Rec}}$ (línea punteada), a diferentes redshifts. La metalicidad $Z_{\text {hot }}$ es definida como el cociente entre la masa de metales contenida en la fase de gas caliente y su masa total; siendo normalizada por el valor solar de $Z_{\odot}=0.0196$ (von Steiger \& Zurbuchen, 2016). En todos los modelos, 
galaxias con altas masas estelares están caracterizadas por gas caliente con mayor enriquecimiento químico, excepto para galaxias con $M_{\star} \gtrsim 5 \times 10^{10} \mathrm{M}_{\odot}$ a bajo redshift $(z \lesssim 0.7)$, donde la tendencia se invierte. Además, la dispersión de $Z_{\text {hot }}$ se vuelve bastante grande para este rango de masa estelar, particularmente para el modelo $\mathrm{SAG}_{\beta 1.9}$.
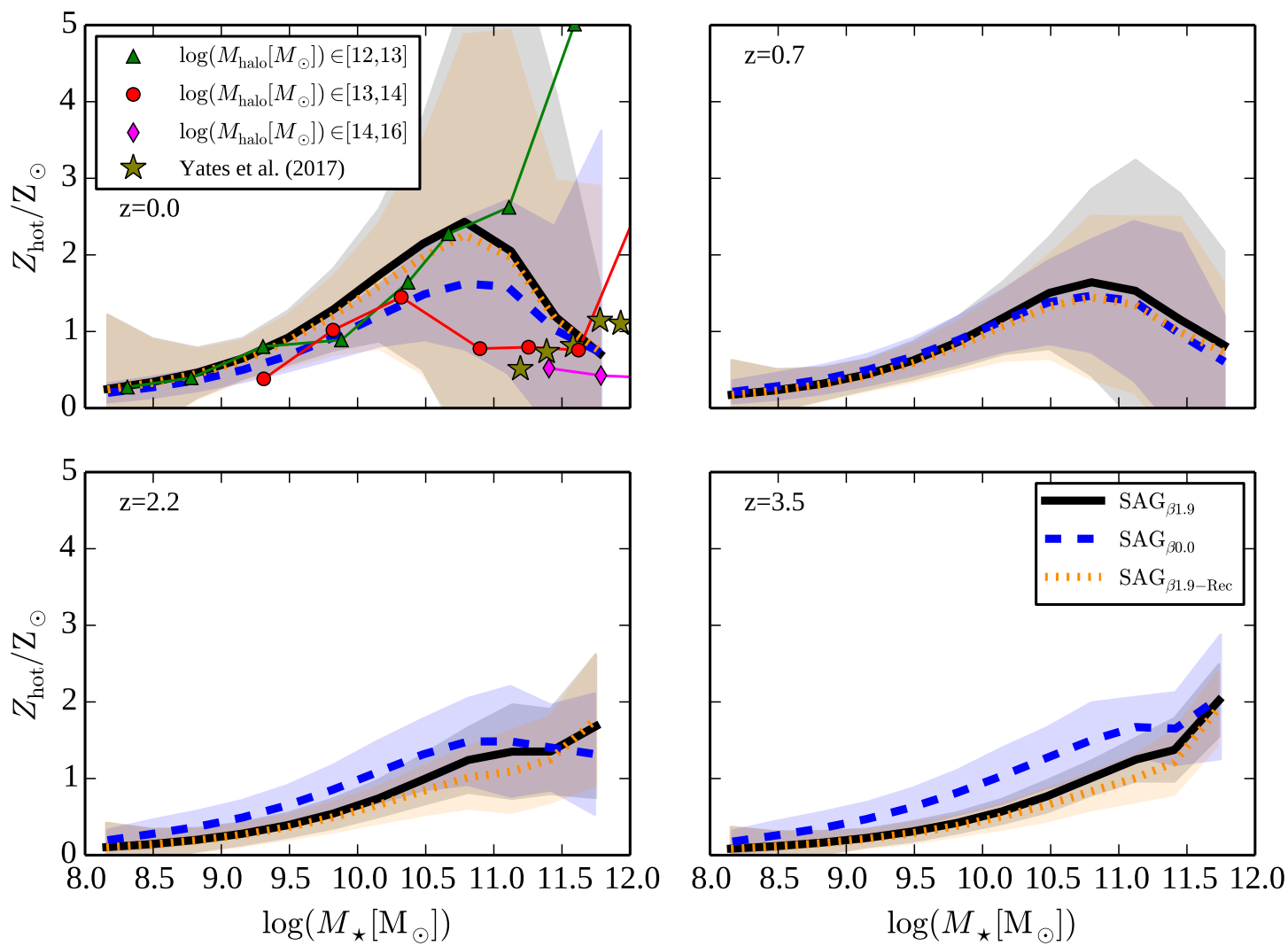

Figura 3.8: Metalicidad del gas caliente $Z_{\text {hot }}$ de galaxias SF como función de la masa estelar a $z=0$ (panel superior izquierdo), $z=0.7$ (panel superior derecho), $z=2.2$ (panel inferior izquierdo) y $z=3.5$ (panel inferior derecho). Las líneas negra sólida, azul a trazos y naranja punteada muestran los valores promedio de la relación para los modelos $\mathrm{SAG}_{\beta 1.9}, \mathrm{SAG}_{\beta 0.0}$ y $\mathrm{SAG}_{\beta 1.9-\mathrm{Rec}}$, respectivamente, mientras que las regiones sombreadas negras, azules y naranjas muestran las correspondientes dispersiones de $1 \sigma$. En el panel superior izquierdo, las metalicidades del gas caliente promedio son estimadas para galaxias generadas por $\mathrm{SAG}_{\beta 1.9}$ en intervalos de $\mathrm{M}_{\text {halo }}: \mathrm{M}_{\text {halo }} / \mathrm{M}_{\odot} \in\left[10^{12}, 10^{13}\right]$ (triángulos verdes), $\mathrm{M}_{\text {halo }} / \mathrm{M}_{\odot} \in\left[10^{13}, 10^{14}\right]$ (círculos rojos) y $\mathrm{M}_{\text {halo }} / \mathrm{M}_{\odot} \in\left[10^{14}, 10^{16}\right]$ (diamantes rosas), lo que ayuda a interpretar la gran dispersión en metalicidad a altas masas estelares. Las estrellas representan los resultados observacionales de Yates et al. (2017) luego de realizar la conversión al plano $Z_{\mathrm{hot}}-M_{\star}$.

Enfocándonos en la forma en que el gas caliente incrementa su metalicidad, puede verse que, independientemente de su masa estelar, el gas caliente de las galaxias $\mathrm{SF}$ en el modelo $\mathrm{SAG}_{\beta 0.0}$ presenta un mayor grado de enriquecimiento químico que la componente caliente de las galaxias en $\mathrm{SAG}_{\beta 1.9}$ a alto redshift $(z \gtrsim 2.2)$. A medida que el redshift decrece, hay una inversión de la tendencia, con $\mathrm{SAG}_{\beta 1.9}$ exhibiendo un gas caliente más contaminado que $\mathrm{SAG}_{\beta 0.0}$. Tal inversión es el resultado de una tasa de enriquecimiento químico del halo de gas caliente diferente entre estos dos modelos a medida que la masa estelar de sus galaxias asociadas crece. En $\mathrm{SAG}_{\beta 1.9}$, la metalicidad del gas caliente incrementa más gradualmente que en $\mathrm{SAG}_{\beta 0.0}$ debido al progresivo decrecimiento de la masa recalentada (eyecciones de gas) con el redshift, especialmente para galaxias masivas (ver Figura 3.6), lo que da lugar a valores más grandes de $f_{\text {recycled }}^{\text {cold }}$ (ver Figura 3.7). Por lo tanto, en $\mathrm{SAG}_{\beta 1.9}$, los me- 
tales reciclados y recientemente sintetizados que son inyectados en el halo de gas caliente a través de eyecciones producen un menor grado de dilución en el material saliente a medida que el redshift decrece. Más aún, el incremento más gradual del $Z_{\text {hot }}$ en $\mathrm{SAG}_{\beta 1.9}$ es favorecido por niveles más bajos de formación estelar y, consecuentemente, menores masas de metales producidas a alto redshift con respecto a tanto $\mathrm{SAG}_{\beta 1.3}$ como $\mathrm{SAG}_{\beta 0.0}$ (Figuras 3.4 y 3.5). El gas caliente del modelo $\mathrm{SAG}_{\beta 1.9-\mathrm{Rec}}$ alcanza niveles de enriquecimiento ligeramente por debajo que en $\mathrm{SAG}_{\beta 1.9}$, con dependencias similares en masa estelar y redshift. Esta pequeña diferencia es consistente con una menor contribución directa del material reciclado recibida en esta fase de gas. En cualquier caso, puede verse que la mayoría del oxígeno producido por las estrellas es depositado en el gas caliente (ver Apéndice A).

Por otro lado, para entender la gran dispersión y el decaimiento encontrados de la tendencia en galaxias de alta masa, comparamos nuestros resultados a $z=0$ con los obtenidos por Yates et al. (2017), representados con estrellas en el panel superior izquierdo de la Figura 3.8. Para ello, discriminamos en intervalos de masa de halo a las galaxias centrales de nuestra muestra para poder realizar una mejor comparación con los datos de Yates et al. (2017). Haciendo esto, encontramos que la gran dispersión y el decaimiento encontrados en la relación $Z_{\mathrm{hot}}-M_{\star}$ están fuertemente relacionados con la masa de halo en que las galaxias habitan y están en acuerdo con los datos observacionales (para más detalle, ver el Apéndice B).

Todo este análisis ayuda a desentrañar los aspectos físicos claves en la evolución de la MZR. Por un lado, tenemos dos modelos que difieren en el modelado del feedback de SN pero tienen el mismo esquema de reciclado, es decir, $\mathrm{SAG}_{\beta 1.9}$ y $\mathrm{SAG}_{\beta 0.0}$. El primero produce una evolución de la normalización de la MZR consistente con las observaciones, mientras que el segundo no muestra evolución alguna. Ambos presentan una fracción $f_{\text {recycled }}^{\text {cold }}$ que se separa del valor cero sólo para galaxias de alta masa, apuntando al hecho de que la contaminación del gas frío está principalmente controlada por el enfriamiento radiativo del gas. Por consiguiente, el aspecto clave que explica la evolución de la MZR es la tasa en la que el halo de gas caliente se enriquece químicamente. Tanto las masas de gas eyectado como de metales producidos son una consecuencia directa de la acción del feedback de SN.

Por otro lado, el fuerte cambio en la tendencia de la relación $f_{\text {recycled }}^{\text {cold }}-M_{\star}$ cuando el esquema de reciclado es modificado tiene poco efecto en la evolución de la normalización de la MZR. Dado que esta evolución es mayor cuando el esquema de reciclado $\mathcal{A}$ es adoptado $\left(\approx 0.44\right.$ para $\left.\mathrm{SAG}_{\beta 1.9}\right)$ que cuando se implementa el esquema de reciclado $\mathcal{B}\left(\approx 0.35\right.$ para $\left.\mathrm{SAG}_{\beta 1.9-\mathrm{Rec}}\right)$, podemos afirmar que el diferente grado de evolución entre $\mathrm{SAG}_{\beta 1.9}$ y $\mathrm{SAG}_{\beta 1.9-\mathrm{Rec}}$ es producido por una tasa de enriquecimiento químico más rápida sufrida por el gas frío en el segundo esquema como resultado de los altos valores de $f_{\text {recycled }}^{\text {cold }}$, especialmente para galaxias de baja masa, lo que lleva a alcanzar valores más altos de la metalicidad del gas frío. Para galaxias de alta masa, el enfriamiento radiativo del gas gana relevancia y los valores bajos de $Z_{\text {hot }}$ en $\mathrm{SAG}_{\beta 1.9-\mathrm{Rec}}$ ayudan a recuperar el aplanamiento en el rango de altas masas de la MZR (ver el panel inferior derecho de la Figura 3.3), con un menor acuerdo con las observaciones.

\subsection{Conclusiones sobre la evolución de la MZR}

Hemos analizado las predicciones de la MZR de la última versión del modelo semi-analítico de formación de galaxias SAG, descrito en detalle en C18 y resumido en la Sección 2.1. Las últimas mejoras implementadas en SAG incluyen un modelado robusto de efectos de ambiente a través de la acción de RPS y TS, acoplado a la integración de las órbitas de las galaxias huérfanas, y una eficiencia mayor del feedback de SN a tiempos tempranos debido a una dependencia explícita con el redshift de las masas recalentadas y eyectadas. Esta última mejora es inspirada por los resultados de las simulaciones hidrodinámicas FIRE (Muratov et al., 2015). C18 muestra que la variante de referencia del modelo, referida como $\mathrm{SAG}_{\beta 1.9}$, permite alcanzar un buen acuerdo con los resultados observacionales en un gran rango de propiedades de galaxias a bajo y alto redshift; la SMF a $z=0 \mathrm{y}$ 
$z=2$, la función de SFR, las relaciones de escala entre la fracción de gas y la masa estelar, entre la masa estelar y la masa del halo principal, entre el contenido de gas atómico y estelar y la evolución de la SFRD. En particular, el modelado detallado del enriquecimiento químico no instantáneo convierte a SAG en una herramienta ideal para llevar a cabo el trabajo realizado en este Capítulo.

Reproducir la MZR ha sido un problema de largo tiempo para los SAMs. La importancia del estudio del origen de esta relación y de la forma en la que evoluciona reside en el conocimiento que aporta sobre el enriquecimiento químico de las fases de gas frío y caliente en conexión con la caída (o acreción) y eyecciones de gas.

Resumimos nuestras conclusiones como sigue:

- Un modelo de feedback de SN que resulte en grandes cantidades de masa recalentada y eyectada a alto redshift permite reproducir la evolución observada de la normalización de la MZR. Esta evolución se encuentra en un amplio rango de masas estelares $\left(10^{8} \mathrm{M}_{\odot} \lesssim M_{\star} \lesssim 10^{12} \mathrm{M}_{\odot}\right)$ hasta $z \lesssim 3.5$ para el modelo $\mathrm{SAG}_{\beta 1.9}$, en donde la nueva receta de feedback de SN involucra una dependencia explícita con el redshift (Figuras 3.1 y 3.2). La falta de dicha dependencia, como resulta del viejo esquema de feedback de $\mathrm{SN}$ (es decir, el modelo $\mathrm{SAG}_{\beta 0.0}$ ), impide que la normalización de la MZR evolucione (Figura 3.3). También encontramos que el nuevo esquema de feedback de SN no genera una evolución en la pendiente de la MZR.

- Experimentamos con la dependencia con el redshift de la masa recalentada y eyectada en la nueva receta de feedback de SN (representada por el parámetro $\beta$ ) y encontramos que valores altos de $\beta$ se traducen directamente en una evolución de la normalización de la MZR más fuerte $(\approx 0.44 \mathrm{dex})$. En cambio, la evolución producida por el modelo $\mathrm{SAG}_{\beta 1.3}$, que tiene $\beta=1.3$, no es tan pronunciada como la alcanzada por el modelo $\mathrm{SAG}_{\beta 1.9}$, el cual adopta $\beta=1.99$ (Figura 3.3). Valores más altos del parámetro $\beta$ resultan en efectos de feedback de SN más violentos a redshifts más altos.

- Mayores efectos de feedback a mayor redshift producen mayores discrepancias entre la SFRD modelada y observada, con el primero estando por debajo de los datos recientes por un factor $\approx 3$ (Figura 3.5). Los modelos con efectos más fuertes de feedback de SN producen valores menores de SFR, con la consecuencia de una menor producción de metales, como resulta de la densidad cósmica del contenido de oxígeno en el gas frío (Figura 3.4).

- Un feedback de SN más fuerte a alto redshift lleva a retrasar la contaminación del gas caliente. Para una dada masa estelar, $Z_{\text {hot }}$ incrementa al decaer el redshift en el modelo $S_{A G_{\beta 1.9}}$, en contraste con el modelo $\mathrm{SAG}_{\beta 0.0}$, en donde $Z_{\text {hot }}$ prácticamente permanece igual a través del tiempo (Figura 3.8). Los niveles bajos del enriquecimiento químico del halo de gas caliente a alto redshift en $\mathrm{SAG}_{\beta 1.9}$ son el resultado de los bajos valores de SFR y de las grandes cantidades de masa recalentada que diluyen los metales inyectados en esta fase de gas (Figura 3.6). El lento crecimiento de la metalicidad del gas caliente comparado con la construcción de la masa estelar es finalmente traducido en una evolución de la normalización de la MZR, dado que parte de la contaminación del gas frío tiene lugar a través del enfriamiento radiativo.

- En el modelo SAG, la contaminación de metales del gas frío ocurre tanto a través de inyección directa del material reciclado en el gas frío o por el enfriamiento radiativo del gas caliente enriquecido. Para una dada masa estelar, la relevancia de cada proceso depende del criterio adoptado (esquema de reciclado) para decidir el destino del material reciclado, es decir, la fase fría o caliente del gas. Cuando la fracción de masa reciclada que termina en la fase de gas frío es definida por el cociente entre las masas recalentadas y recicladas (como en los modelos $\mathrm{SAG}_{\beta 1.9}, \mathrm{SAG}_{\beta 1.3}$ y $\mathrm{SAG}_{\beta 0.0}$ ), el enfriamiento radiativo del gas es el principal mecanismo de contaminación de galaxias de baja masa $\left(M_{\star} \lesssim 10^{10} \mathrm{M}_{\odot}\right)$, pero pierde relevancia a medida 
que la masa estelar aumenta. La tendencia opuesta es obtenida cuando el destino del material reciclado es regulado por la fracción de gas frío de las galaxias (Figura 3.7).

- El modelado del enriquecimiento químico de las eyecciones de gas no tiene efecto en nuestras conclusiones. Un experimento en donde el destino del material reciclado es regulado por la fracción del gas frío (modelo $\mathrm{SAG}_{\beta 1.9-\mathrm{Rec}}$, esquema de reciclado $\mathcal{B}$ ), en lugar de ser controlado por la cantidad de masa recalentada en $\mathrm{SAG}_{\beta 1.9}$ (esquema de reciclado $\mathcal{A}$ ), muestra que la población de galaxias exhibe una evolución de la normalización de la MZR que sólo es ligeramente menor $(\approx 0.35 \mathrm{dex})$ que en el modelo fiduciario (Figura 3.3). Esta pequeña diferencia es atribuida a un enriquecimiento más rápido del gas frío en $\mathrm{SAG}_{\beta 1.9-\operatorname{Rec}}$ debido al papel predominante de la inyección directa de metales en esta fase de gas para galaxias de baja masa.

En general, encontramos con SAG que una evolución más fuerte del efecto de feedback de SN es clave para obtener la esperada evolución de la normalización de la MZR, la cual ha sido un desafío para los SAMs. Tanto la masa eyectada como los metales producidos son una consecuencia directa de la acción del feedback de SN. Entonces, la evolución de la normalización de la MZR es principalmente causada por los bajos niveles de formación estelar, y la consecuente baja producción de metales, como resultado de un feedback de SN más fuerte a alto redshift, con menor dependencia del destino de los metales producidos por la evolución estelar y devueltos al medio por vientos estelares y SNs. La explícita dependencia con el redshift involucrada en la estimación de las eyecciones generadas por el feedback de SN es interpretada como aspectos del ISM que el modelo no logra capturar, como la diferente evolución con el redshift de las propiedades del disco local con respecto a la velocidad circular de las galaxias (Lagos et al., 2013; Creasey et al., 2013), o fuentes adicionales de energía (además de las SNs) que pueden jugar un papel importante (Hopkins et al., 2012). 


\section{Capítulo 4}

\section{El efecto de la Tasa de Gas Acretado en los Perfiles Radiales de Abundancia}

Los perfiles radiales de abundancia del gas, también conocidos como perfiles radiales de metalicidad (RMPs), muestran la cantidad de metales en la fase gaseosa que una galaxia tiene a una cierta distancia respecto de su centro. Esta relación tiende a decrecer con el radio, mostrando mayor cantidad de metales en las regiones centrales de las galaxias que en las regiones externas, a su vez que exhibe un aplanamiento en estas últimas. Sin embargo, los estudios realizados con la técnica de IFS de la última década han mostrado una variedad importante en la forma de esta relación, incluso una inversión de la misma (es decir, más metales en las regiones externas que en las internas) a alto redshift (ver Sección 1.2.2 para más detalles de la discusión). Estos cambios en los perfiles resultan, entonces, estar relacionados con los procesos físicos involucrados en la evolución química de las galaxias. De esta manera, el estudio de la forma de los perfiles radiales de metalicidad permite tener un mejor panorama del entendimiento de la formación y evolución de las galaxias.

En este Capítulo, describimos el estudio del efecto que tienen distintas propiedades de las galaxias sobre sus perfiles radiales de metalicidad del gas hasta $z \leq 1$, con el fin de esclarecer un poco los distintos aspectos de este tema que estan sujetos a debate. En particular, nos enfocamos en el efecto que produce la tasa de acreción de gas y comprobamos su importancia en este estudio. También estudiamos la relevancia de propiedades como la masa estelar, la tasa de formación estelar y la fracción de gas sobre estos perfiles. Los resultados mostrados en este Capítulo han sido publicados en Collacchioni et al. (2020).

Debido a que necesitamos estudiar a las galaxias como objetos extendidos, para este estudio utilizamos galaxias simuladas de las simulaciones hidrodinámicas EAGLE descritas en la Sección 2.2.3. A menos que se estipule lo contrario, mantenemos el análisis de las muestras de las dos simulaciones por separado. Recordamos que la simulación Recal-L25N752 tiene parámetros ligeramente diferentes de feedback estelar y AGN, debido a que esta corrida intenta reproducir a $z=0.1$ la función de masa estelar con una resolución mayor (ver la discusión de convergencia fuerte y débil en la Sección 2.2.4).

\subsection{Muestra de galaxias simuladas}

Debido a lo sensible que es la caída de gas a los efectos de ambiente como TS, RPS, interacciones, etc., los cuales afectan principalmente a las galaxias satélites, nos limitamos al estudio de las galaxias SF centrales (es decir, galaxias que se encuentran en los centros de los pozos de potencial de los halos). A su vez, aplicamos la restricción de considerar sólo galaxias con $M_{\star} \geq 10^{9} \mathrm{M}_{\odot}$ para la simulación Recal-L25N752 y $M_{\star} \geq 10^{10} \mathrm{M}_{\odot}$ para la simulación Ref-L100N1504, debido a sus respectivas resoluciones (lo que da un mínimo en el número de partículas estelares de $\approx 6700 \mathrm{y}$ $\approx 7700$, respectivamente). De estas galaxias centrales incluimos aquéllas cuya tasa de gas acretado 
$\dot{M}_{\text {accr }}$ (definida en la Sección 4.2) corresponde al menos a 10 partículas de gas SF, lo que se traduce en $\dot{M}_{\text {accr }} \approx 9 \times 10^{-2} \mathrm{M}_{\odot} \mathrm{yr}^{-1}$ a $z=0$ y $\dot{M}_{\text {accr }} \approx 5 \times 10^{-2} \mathrm{M}_{\odot} \mathrm{yr}^{-1}$ a $z=1$. En el Apéndice C, testeamos nuestros resultados principales con diferentes elecciones de este número mínimo de partículas SF, y encontramos que el límite es insensible hasta 100 partículas. Nuestros resultados son, así, bien convergentes sobre este límite.

Nuestra muestra final cuenta con un total de 1280 galaxias a $z=0$ y 1642 a $z=1$. Debido a la evolución de tamaños de las galaxias (galaxias a alto redshift son más pequeñas a masa estelar fija, Furlong et al. 2017), nos limitamos a estudiar hasta $z \leq 1$, donde las galaxias están mejor resueltas. Calculando los valores medianos de los radios a mitad de masa estelar, $r_{50}$, para toda la muestra a cada redshift y para cada simulación, encontramos que los tamaños físicos promedios de nuestras galaxias seleccionadas en la simulación Recal-L25N752 son $3.98 \mathrm{kpc}$ y $3.17 \mathrm{kpc}$ a redshifts $z=0$, y $z=1$, respectivamente. De manera similar, los tamaños físico promedios de galaxias de la muestra de la simulación Ref-L100N1504 son $4.22 \mathrm{kpc}$ y $3.11 \mathrm{kpc}$ a redshifts $z=0$ y $z=1$, respectivamente. Cabe recordar que las galaxias de esta simulación son más masivas que las seleccionadas en RecalL25N752. En ambos casos, los tamaños están cómodamente por encima del suavizado gravitacional (o softening) de cada correspondiente simulación (ver Tabla 2.2). Ludlow et al. (2019b) mostraron que la resolución espacial de una simulación es aproximadamente 0.05 veces el espaciado medio de las partículas, el cual equivale a 66.5 pkpc para Ref-L100N1504 y 33.35 pkpc para Recal-L25N752 a $z=0$, obteniendo así radios mínimos de convergencia de $3.3 \mathrm{kpc}$ y $1.6 \mathrm{kpc}$, respectivamente. Esto significa que para que una galaxia simulada resulte resuelta debe tener un radio mayor a estos radios de convergencia, monstrando que la selección de masa estelar aplicada aquí contiene galaxias resueltas de ambas simulaciones.

\subsection{Cálculo de la tasa de acreción de gas}

Computamos la tasa de acreción de gas, $\dot{M}_{\text {accr }}$, que cae a las galaxias usando una metodología simple de rastreo de partículas (siguiendo a Neistein et al. 2012). Para un dado subhalo a una dada salida de la simulación, primero identificamos las partículas de gas SF, como se describe en la Sección 2.2.2. Luego, definimos las partículas de gas que se acretarán como el conjunto que cumple las siguientes condiciones:

(a) estar ligadas al progenitor principal del subhalo en la salida anterior en su árbol de fusión, y

(b) no ser SF en ese tiempo.

Las partículas de estrellas que satisfacen las condiciones $(a)$ y $(b)$ también son tenidas en cuenta en el cálculo del gas acretado. Estas partículas estelares son aquéllas que vienen de partículas de gas que fue acretado, pero que se transformaron en estrellas más rápido que el resto del gas. Con este criterio, nos aseguramos que el gas acretado venga del mismo subhalo estudiado y, al mismo tiempo, que la acreción misma cambie el estado del gas (de no SF a SF). En otras palabras, estamos interesadas en la caída de gas que pueda desencadenar la formación estelar. Esto último se hace para conectar más fácilmente con las observaciones, las cuales usan, entre otros métodos, líneas de emisión nebular como trazadores de gas y para estimar la metalicidad del mismo.

La elección de sólo considerar el gas que viene del progenitor principal es para calcular la contribución del gas acretado que viene del medio circumgaláctico (CGM, por Circumgalactic Medium) difuso en lugar del que viene por fusiones de galaxias. Remarcamos que en simulaciones hidrodinámicas previas se muestra que esta "acreción suave" es la fuente principal de la acreción de gas de las galaxias (van de Voort et al., 2011). Wright et al. (2020) muestra que en EAGLE el $80 \%$ o más del gas acretado viene de una acreción suave (lo que incluye tanto acreción de gas prístino como gas pre-procesado). La $\dot{M}_{\text {accr }}$ es, entonces, simplemente definida como la masa del gas acretado que satisface las condiciones $(a)$ y $(b)$, dividido el intervalo de tiempo entre las salidas de la simulación. Los subhalos progenitores son identificados usando los árboles de fusión con el algoritmo DHaLo (Jiang 
et al., 2014; Qu et al., 2017).

Para la simulación Ref-L100N1504, usamos un conjunto de 50 salidas para computar la $\dot{M}_{\text {accr }}$, mientras que para Recal-L25N752 usamos un conjunto de 29 salidas. La razón por la cual estos números son distintos es que para Ref-L100N1504 se tiene a disposición un mayor número de salidas con un mejor espaciado temporal aunque se reduce el número de propiedades de las partículas de gas), en comparación con las 29 salidas por defecto de todas las simulaciones del proyecto que son de acceso público en la base de datos de EAGLE (McAlpine et al., 2017).

El intervalo de tiempo entre las salidas de Ref-L100N1504 es de 0.42 Gyr para $z=0 \rightarrow 0.03$ y 0.4 Gyr para $z=1 \rightarrow 1.05$, mientras que para Recal-L25N752 el intervalo de tiempo entre las salidas es de 1.34 Gyr para $z=0 \rightarrow 0.1$ y 0.93 Gyr para $z=1 \rightarrow 1.26$. Aunque estemos usando pasos de tiempo distintos para calcular las tasas de acreción en las dos simulaciones, esto no tiene un impacto importante en nuestros resultados debido a que la acreción pasa en escalas de tiempo largas (Mitchell et al., 2020; Wright et al., 2020). Si estuviéramos estudiando eyecciones de gas, sin embargo, esta diferencia de tiempo sería importante, dado que las eyecciones pasan en escalas de tiempo cortas y son más estocásticas.

Sin embargo, hay una debilidad inherente al método de rastreo de las partículas. Si hay una caída de gas que se une al gas SF del ISM pero lo abandona antes que la acreción de gas sea definida en esa salida (con "abandona" nos referimos a que las partículas son transferidas a otra fase, como por ejemplo el ISM no-SF), entonces esa caída no será tenida en cuenta.

\subsection{Estimación de los perfiles radiales de metalicidad del gas}

Dado que estamos interesadas en estudiar cómo los perfiles de metalicidad de gas son alterados por la $\dot{M}_{\text {accr }}$, necesitamos definir la manera en la que los perfiles son calculados. A menos que se diga lo contrario, consideramos las partículas de gas SF en una cáscara esférica que incrementa en intervalos físicos de $1 \mathrm{kpc}$ desde 1 a $9 \mathrm{kpc}$, seguido de intervalos de $3 \mathrm{kpc}$ hasta $51 \mathrm{kpc}$, por intervalos de $10 \mathrm{kpc}$ hasta $101 \mathrm{kpc}$, y finalmente intervalos de $50 \mathrm{kpc}$ hasta el radio de valor $501 \mathrm{kpc}$. Esto se hace para evitar el ruido causado por la densidad de las partículas SF que decrecen abruptamente con el radio. La metalicidad del gas suavizado (o smoothed, S15, Trayford \& Schaye 2019) contenida dentro de cada cáscara será el cociente entre la masa de los metales más pesados que el helio sobre la masa total de gas, es decir

$$
Z=\frac{M_{\text {metals }}}{M_{\mathrm{H}}+M_{\mathrm{He}}+M_{\text {metals }}},
$$

donde $M_{\mathrm{H}}, M_{\mathrm{He}} \mathrm{y} M_{\text {metals }}$ son las masas de hidrógeno, helio y metales de las partículas de gas SF, respectivamente. Usamos una escala logarítmica $\left(\log _{10}\left(Z / Z_{\odot}\right)\right)$ para expresar la metalicidad en una cierta cáscara, donde $Z_{\odot}$ es la metalicidad solar (aquí adoptamos el valor $Z_{\odot}=0.0127$, Asplund et al. 2005). Esta forma de calcular la metalicidad es común en los trabajos teóricos. Notamos, sin embargo, que el cálculo del cociente oxígeno sobre hidrógeno en su lugar (comúnmente usado en trabajos observacionales) deriva efectivamente en los mismos resultados (ver Apéndice D para una comparacón entre resultados observacionales y de otras simulaciones con los de esta Tesis).

\subsection{La conexión entre la metalicidad, la masa estelar y la $\dot{M}_{\text {accr }}$}

La Figura 4.1 muestra MZR a redshifts $z=0$ (panel superior) y $z=1$ (panel inferior) para la muestra combinada de galaxias de las simulaciones Recal-L25N752 y Ref-L100N1504. En este caso, la metalicidad del gas es estimada a partir de todas las partículas de gas SF dentro de un radio de $30 \mathrm{kpc}$ respecto del centro del pozo de potencial de cada galaxia (este resulta ser el radio típico en el que las propiedades integradas de las galaxias son calculadas en las simulaciones EAGLE; ver S15 
para más detalle). Los valores medios de la metalicidad son mostrados con líneas sólidas, mientras que las líneas a trazos representan los percentiles $16-84$. El degradé de colores denota los valores medios de la $\dot{M}_{\text {accr }}$ de las galaxias en intervalos de 0.25 dex en masa estelar y metalicidad. En los trabajos observacionales, las metalicidades del gas son usualmente estimadas de regiones que pueden ser más pequeñas que $30 \mathrm{kpc}$. Por esta razón, investigamos el efecto de elegir una apertura más chica $(10,15$ y $20 \mathrm{kpc})$ y encontramos que los cambios en la MZR son despreciables, excepto en el extremo masivo $\left(M_{\star}>10^{11} \mathrm{M}_{\odot}\right)$ donde vemos un incremento en la dispersión. Estas diferencias, sin embargo, son pequeñas $\mathrm{y}$, por lo tanto, decidimos continuar nuestro análisis con una apertura de $30 \mathrm{kpc}$ para facilitar la comparación con otros trabajos teóricos.

Como es esperado, la metalicidad del gas incrementa con la masa estelar hasta un límite de masa en donde la metalicidad se satura o incluso decrece. Este límite de masa incrementa con el redshift desde $\approx 10^{10} \mathrm{M}_{\odot}$ a $z=0$ hasta $10^{11} \mathrm{M}_{\odot} \mathrm{a} z=1$. Esto resulta relacionado con la transición de galaxias que son preferentemente SF a preferentemente pasivas. A alto redshift, esto sucede a mayor masa estelar que a $z=0$, como se muestra en el desarrollo de la secuencia roja en EAGLE (Trayford et al., 2016; Wright et al., 2019). A $z=1$, Trayford et al. (2016) mostraron que la secuencia roja en EAGLE es poblada en el extremo masivo por galaxias centrales y a bajas masas $\left(M_{\star}<10^{9.5} \mathrm{M}_{\odot}\right)$ por galaxias satélites, mostrando una escasez de galaxias alrededor de $10^{10} \mathrm{M}_{\odot}$. Esta región de escasez incrementa su población hacia $z=0$. Trayford et al. (2016) y Bower et al. (2017) argumentaron que esto se debe a que el feedback de AGN es efectivo sólo para galaxias de alta masa a $z=1$, que es cuando los BHs han crecido lo suficiente para ser eficientes en generar eyecciones de material. La combinación del efecto del AGN y el bajo número de galaxias SF lleva a un aplanamiento de la MZR, desplazándose de masas mayores a menores a medida que el tiempo pasa, e incluso puede aparecer como un decrecimiento de la metalicidad en el extremos masivo. Este último ha sido reportado en observaciones (Sánchez et al., 2017), y puede deberse en parte a incertezas sistemáticas. En galaxias muy ricas en metales, la temperatura electrónica decae, complicando y generando subestimaciones de la metalicidad (Kewley \& Ellison, 2008). Si sumamos a este escenario el hecho de que galaxias masivas también presentan mayor $\dot{M}_{\text {accr }}$ a cada redshift, entonces esta caída de gas podría estar diluyendo la metalicidad del gas, contribuyendo al decaimiento visto en la Figura 4.1. En el extremo poco masivo, las satélites sólo aportan una pequeña contribución al número total de galaxias y, por lo tanto, no impactan en la forma de la MZR.

La normalización de la MZR también incrementa con el decrecimiento del redshift, y su forma (en términos de la pendiente de la relación) también cambia con el redshift. Entonces, la MZR evoluciona en normalización y forma, implicando que la masa estelar de las galaxias y la abundancia química evolucionan con ritmos diferentes. Esto ha sido reportado por Guo et al. (2016) para la simulación Ref-L100N1504 y por De Rossi et al. (2017) para la Recal-L25N752. Esta evolución en la normalización es un suceso natural en las simulaciones EAGLE y en general no es de fácil obtención en otras simulaciones (como vimos en los Capítulos 1 y 3). Es interesante notar que, a redshift fijo, las galaxias con mayor masa estelar también tienen mayor $\dot{M}_{\text {accr }}$, en promedio. Esta correlación positiva es ajustada (con una dispersión de $z \sim 0.5$ dex) en todos los redshifts analizados aquí, y está en acuerdo con trabajos teóricos previos (ejemplos, van de Voort et al. 2011; Mollá et al. 2016; Correa et al. 2018; ver también Apéndice E).

De la Figura 4.1 también puede verse que, a masa estelar fija, la metalicidad del gas incrementa con el decrecimiento de la $\dot{M}_{\text {accr }}$, independientemente del redshift considerado. Las líneas grises gruesas denotan la MZR de galaxias en diferentes rango de $\dot{M}_{\text {accr }}$ (como se describe en las leyendas). Las galaxias con valores de $\dot{M}_{\text {accr }}$ menores tienen una MZR con una normalización mayor, es decir, con valores de metalicidad mayores. Esta tendencia se asemeja a la FMR observada (Mannucci et al., 2010; Lara-López et al., 2010), la cual correlaciona la metalicidad, la masa estelar y la SFR de forma que a masa estelar fija la metalicidad incrementa a medida que la SFR decrece. La existencia de esta relación en EAGLE fue demostrada y discutida en De Rossi et al. (2017) y Matthee \& Schaye (2018). 
En este escenario, la acreción de gas prístino tiene un efecto dual de dilución de la metalicidad del gas y disparador de la formación estelar (Troncoso et al., 2014). Sin embargo, el hecho de que De Rossi et al. (2017) encontraran una fuerte anti-correlación entre la sSFR y la metalicidad en simulaciones con feedback estelares más fuertes indica que las eyecciones de material (que preferentemente eyectan metales) también juegan un rol importante.

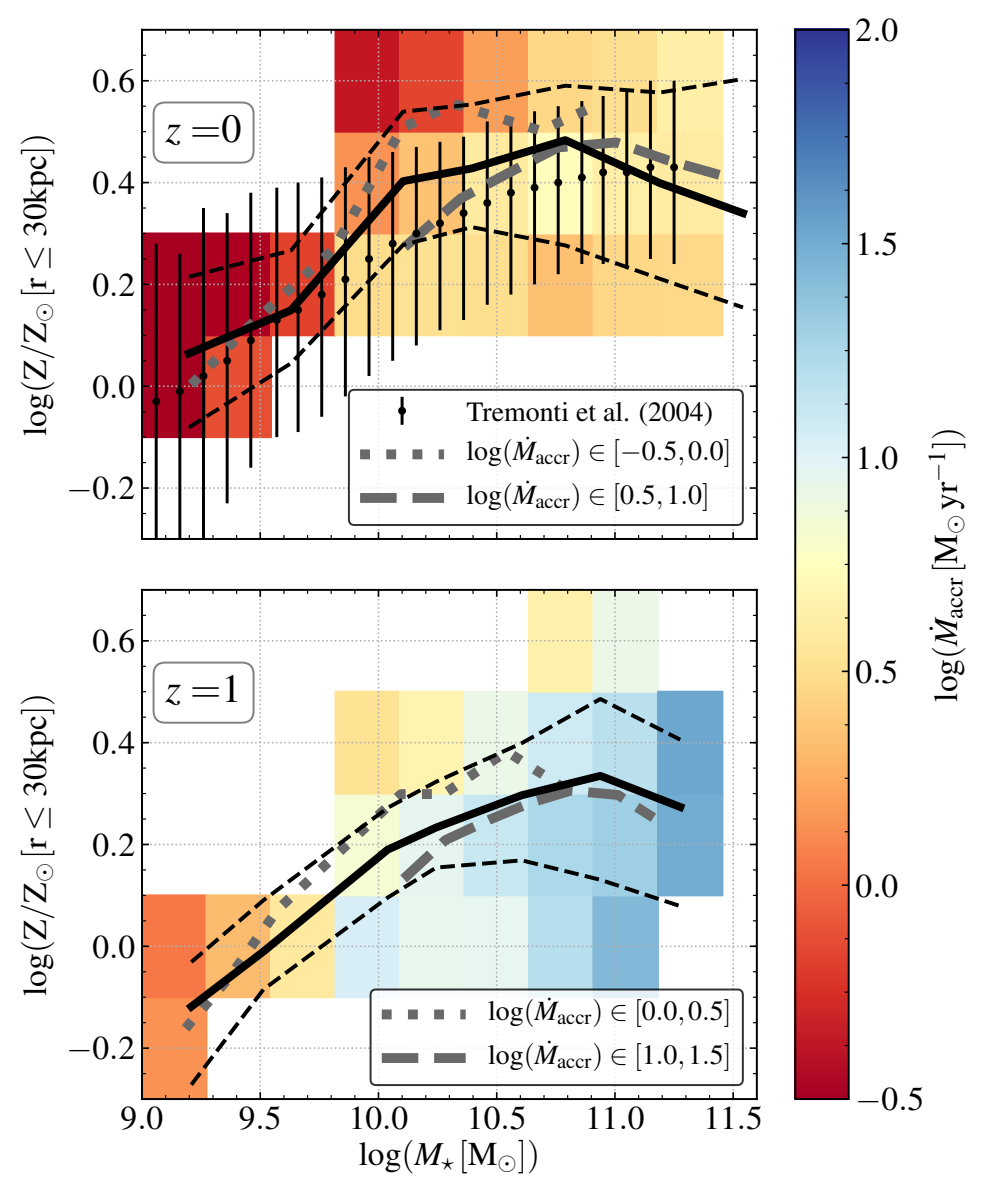

Figura 4.1: Relación masa estelar-metalicidad del gas (MZR) a $z=0$ (panel superior) y $z=1$ (panel inferior) para galaxias en la muestra simulada combinada (considerando simultáneamente la muestra de galaxias de las simulaciones Recal-L25N752 y Ref-L100N1504). La metalicidad del gas es estimada usando todas las partículas de gas SF dentro de $30 \mathrm{kpc}$ del centro de potencial de la galaxia. En cada panel, las líneas negras sólidas y a trazos finas representan la mediana y los percentiles 16 - 84, respectivamente. Los símbolos negros con barras de error en el panel superior muestran las mediciones observacionales de la MZR de Tremonti et al. (2004). A un dado redshift, las galaxias con mayor masa estelar muestran mayor metalicidad. A una dada masa estelar, las galaxias muestran mayor metalicidad a medida que el redshift decrece. Los píxeles en color muestran la mediada de la $\dot{M}_{\text {accr }}$ de las galaxias en intervalos de masa estelar y metalicidad (que contienen al menos 10 galaxias). Galaxias con mayor masa estelar muestran mayor $\dot{M}_{\text {accr }}$, con valores de $\dot{M}_{\text {accr }}$ decreciendo al aumentar el redshift. Las líneas grises finas punteada y a trazos denotan la mediana de las galaxias en dos intervalos de $\dot{M}_{\text {accr }}$, como se muestra en los paneles (presentado en unidades de $\mathrm{M}_{\odot} \mathrm{yr}^{-1}$ ). A una masa estelar y redshift fijos, hay una tendencia de anti-correlación entre la metalicidad del gas y la $\dot{M}_{\text {accr. }}$.

Las galaxias presentan valores menores de $\dot{M}_{\text {accr }}$ a medida que el redshift decrece (a masa estelar 
fija), mostrando una evolución con el tiempo. Esto resulta esperado al ser la acreción de gas en los halos y, por lo tanto en las galaxias, decreciente con el tiempo (Correa et al., 2018). Sin embargo, es importante enfatizar que se esperan grandes discrepancias entre la $\dot{M}_{\text {accr }}$ que cae a los halos y la que cae a las galaxias (ejemplos, van de Voort et al. 2011; van de Voort 2017; Nelson et al. 2013, 2019). El juego entre la $\dot{M}_{\text {accr }}$ y la SFR será analizado en la Sección 4.5.3.

\subsection{Efecto de la $\dot{M}_{\text {accr }}$ en los gradientes de metalicidad del gas}

En esta Sección, presentamos las correlaciones encontradas entre varias propiedades de galaxias obtenidas por las simulaciones EAGLE Ref-L100N1504 y Recal-L25N752, a saber, la masa estelar, la $\dot{M}_{\text {accr }}$, la SFR y la fracción de gas neutro, y cómo se correlacionan con los RMPs. Esto se hace a $z=0$ y 1 . El análisis también se ha realizado en redshifts intermedios, produciendo resultados consistentes. Por consiguiente, mostramos sólo los resultados obtenidos para $z=0,1$ por simplicidad.

\subsubsection{La relación entre RMP y $\dot{M}_{\text {accr }}$}

El foco de esta Sección es tener un mejor entendimiento de cómo la $\dot{M}_{\text {accr }}$ y otras propiedades galácticas afectan los RMPs. Como se discutió en el Capítulo 1, la pendiente del RMP medida sobre toda la galaxia no depende de la masa estelar o del ambiente en donde la galaxia está inmersa, particularmente en las simulaciones EAGLE (Tissera et al., 2019). Sin embargo, dado que estamos interesadas en investigar si hay un radio preferencial por encima/debajo del cual los perfiles se ven significativamente afectados por la acreción de gas, dividimos las galaxias en dos regiones y analizamos las correspondientes pendientes en lugar de tomar una sola pendiente del RMP. Medimos la pendiente de la ley de potencia que ajusta el RMP, $\alpha$, en regiones definidas por el radio que contiene la mitad de la masa estelar de la galaxia, referido como $r_{50}{ }^{1}$. El beneficio de usar este radio es que obtenemos $\alpha$ en regiones que se consideran "internas" o "externas", independientemente del tamaño físico de la galaxia. Así, medimos $\alpha$ en dos regiones: $r / r_{50}<1$ y $1<r / r_{50}<5$. Nos referimos a estas regiones como interna y externa, respectivamente. Para encontrar $\alpha$, usamos un ajuste lineal en el espacio $\log \left(Z / Z_{\odot}\right)-r$, como sigue

$$
\log _{10}\left(Z / Z_{\odot}\right)=\alpha \times r+\log _{10}\left(Z_{0} / Z_{\odot}\right)
$$

donde $Z$ es la metalicidad del gas definida en la Ecuación 4.1, $\alpha$ es la pendiente que queremos estudiar (en unidades de dex $r_{50}^{-1}$, lo que permite estudiar el cambio en la pendiente del RMP con respecto a las propiedades galácticas independientemente de la escala física de las galaxias), y $Z_{0}$ es la normalización del ajuste. Aquí nos concentramos en $\alpha$ dado que la normalización de la metalicidad del gas en galaxias ya ha sido estudiada en una variedad de trabajos previos con las simulaciones EAGLE (ejemplos, Lagos et al. 2016; De Rossi et al. 2017).

Tissera et al. (2019) encontraron, también utilizando EAGLE, que la pendiente del perfil de metalicidad del gas correlaciona fuertemente con el radio mitad de masa de gas neutro (es decir, el radio que contiene la mitad de la masa de gas atómico y molecular juntos), aunque el radio mitad de masa estelar no juega un rol relevante. Esto sugiere que el radio efectivo del contenido de gas neutro sería el más apropiado para estudiar los RMP. Sin embargo, esta propiedad se encuentra raramente disponible en observaciones e incluso es más difícil de definir robustamente. Por ejemplo, observaciones de HI han mostrado que integraciones más largas, lo que permite encontrar columnas de densidad de HI más bajas, continúa revelando cantidades de HI, haciendo que el radio mitad de masa del gas sea dependiente de la sensibilidad del instrumento (ejemplos, Oosterloo et al. 2007; Heald et al. 2011;

\footnotetext{
${ }^{1}$ La motivación de tomar el radio $r_{50}$ surge por ser un dato que sale de las simulaciones (es decir, ya se encuentra calculado; McAlpine et al. 2015) y, por otro lado, evoca al radio efectivo definido en fotometría como el radio que encierra la mitad de flujo integrado, utilizado, por ejemplo, en la Ley de Sérsic (Sersic, 1968; Sparke \& Gallagher, 2007).
} 
Kamphuis et al. 2013). El $r_{50}$ estelar es una cantidad bien definida que puede ser medida robustamente para cientos de miles de galaxias (ver Lange et al., 2016, para un ejemplo en el Universo local).

La Figura 4.2 muestra la relación entre la pendiente del RMP en la región interna (paneles izquierdos) y externa (paneles derechos) de las galaxias de EAGLE como función de la $\dot{M}_{\text {accr }}$ a $z \leq 1$. Las líneas sólidas y a trazos muestran los valores medios de la relación y los percentiles 16 - 84, respectivamente. Los resultados de ambas simulaciones se muestran por separado usando diferentes colores. Hay un pequeño desplazamiento entre las simulaciones en esta Figura, que no resulta sorprendente dado que para Recal-L25N752 estamos incluyendo todas las galaxias de masa estelar $M_{\star} \geq 10^{9} \mathrm{M}_{\odot}$, mientras que para la simulación Ref-L100N1504 nos limitamos a una muestra de galaxias con $M_{\star} \geq 10^{10} \mathrm{M}_{\odot}$. En las siguientes Subsecciones mostramos que, al eliminar la dependencia con la masa estelar, ambas simulaciones llegan a acuerdos.
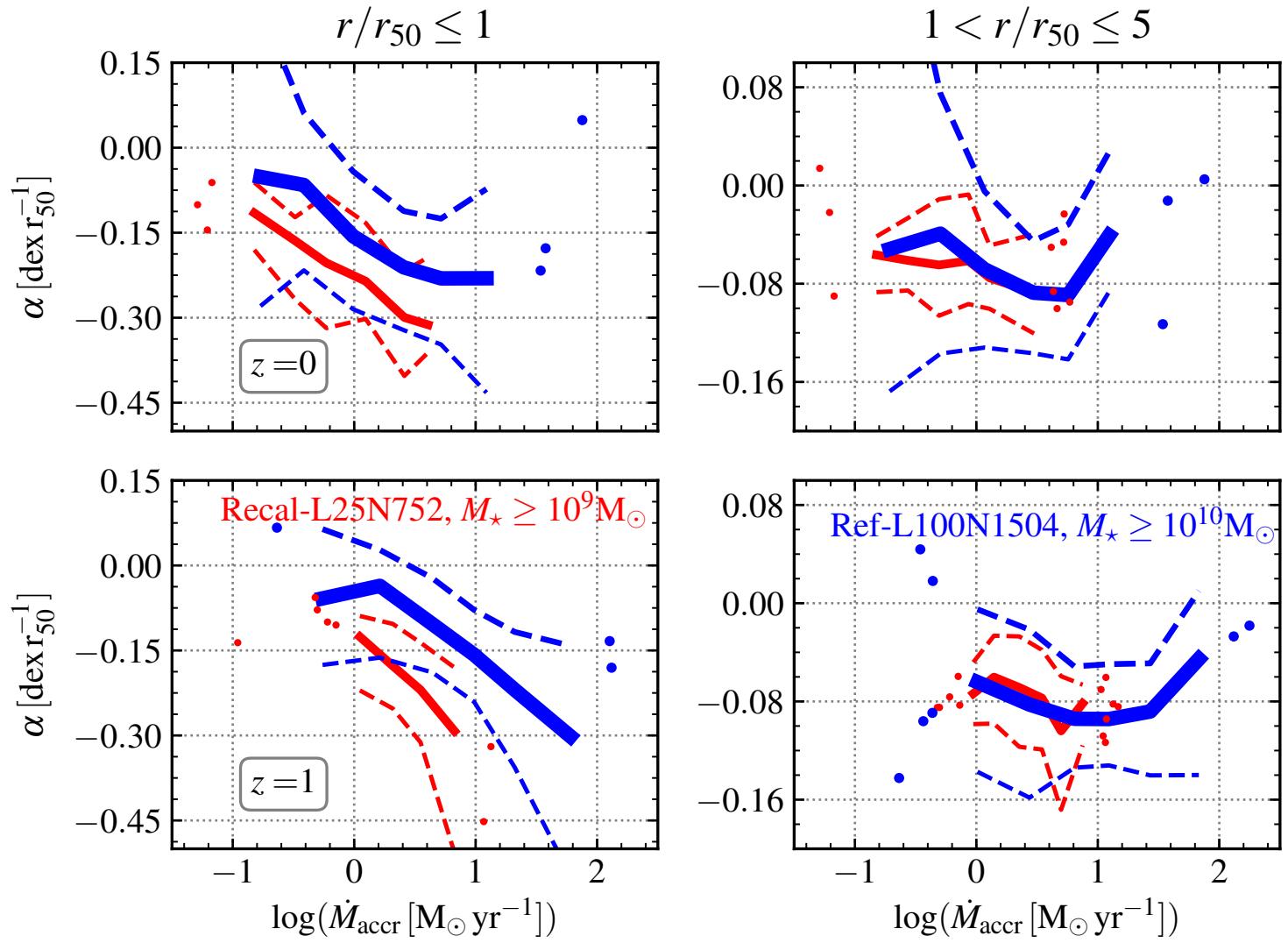

Figura 4.2: Valores medios de las pendientes de los RMPs, $\alpha$, por cada simulación usada en este capítulo como función de la $\dot{M}_{\text {accr }}$. Los paneles superiores e inferiores muestran las galaxias a $z=0$ y $z=1$, respectivamente. Los paneles izquierdos y derechos denotan diferentes regiones de las galaxias, como se muestra en la figura. Las líneas a trazos representan los percentiles 16 - 84. Galaxias individuales se muestran donde los intervalos tienen menos de 10 objetos (círculos). La pendiente del RMP en la región interna $\left(r / r_{50} \leq 1\right)$ es negativa y está fuertemente correlacionada con la $\dot{M}_{\text {accr }}$ para ambas simulaciones a todo redshift analizado, siendo más negativa para $\dot{M}_{\text {accr }}$ mayores. La simulación Recal-L25N752 muestra una pendiente más negativa, especialmente para

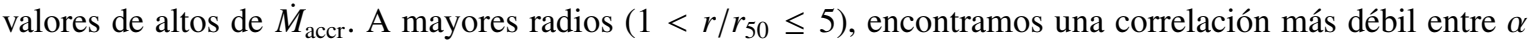
y $\dot{M}_{\text {accr }}$, con valores de $\alpha \approx-0.08$. Como se explica en la Sección 4.1, los cortes en masa estelar para nuestra muestra de galaxias es $M_{\star} \geq 10^{9} \mathrm{M}_{\odot}$ para Recal-L25N752 y $M_{\star} \geq 10^{10} \mathrm{M}_{\odot}$ para Ref-L100N1504. Notar que los rangos del eje $y$ cambian de los paneles izquierdos a los derechos, con el fin de resaltar los valores alcanzados por nuestros datos.

Para la región interna puede verse que las galaxias con mayor $\dot{M}_{\text {accr }}$ presentan pendientes del 
RMP $\alpha$ más negativas, con una dispersión de $\approx 0.1-0.2 \operatorname{dex} r_{50}^{-1}$. La anti-correlación entre $\alpha$ y $\dot{M}_{\text {accr }}$ está presente en ambas simulaciones, aunque la tendencia es más fuerte para Recal-L25N752. Esta tendencia se observa a todo redshift estudiado. Una explicación física simple para interpretar esta tendencia sería que el gas acretado tiene menor metalicidad que el ISM de la galaxia y, entonces, diluye el gas que está cayendo al disco. Esto haría más inclinado el RMP debido a que la conservación del momento angular específico causaría que el gas acretado se asiente en las partes externas de las galaxias (ver Tissera et al., 2019, para un análisis de este escenario en EAGLE). Otra explicación es que la correlación en la Figura 4.2 puede estar causada por estas dos propiedades galácticas correlacionando con una tercera, más fundamental, lo cual se explora en las siguientes Secciones.

En las regiones externas, $1<r / r_{50}<5$, encontramos que $\alpha$ permanece con valores cercanos a $\alpha \approx-0.08 \operatorname{dex} r_{50}^{-1}$, con una dispersión de $\approx 0.1$ dex. Es interesante notar que los valores de $\alpha$ en esta región son significativamente menos negativos que para la pendiente del RMP en las partes internas de las galaxias que tienen mayor $\dot{M}_{\text {accr }}$, mientras que galaxias con menor $\dot{M}_{\text {accr }}$ tienen valores similares de pendiente en ambas regiones. Hay una débil tendencia de $\alpha$ de ser bivaluada con $\dot{M}_{\text {accr }}$ en las regiones externas, con valores de $0.5 \leq \dot{M}_{\mathrm{accr}}\left[\mathrm{M}_{\odot} \mathrm{yr}^{-1}\right] \leq 1.5$ asociados a pendientes externas más negativas. Sin embargo, este efecto es débil, con diferencias de $\lesssim 0.05$ dex en $\alpha$ y sólo apreciable en la simulación Ref-L100N1504. La gran dispersión es probablemente debida al bajo número de partículas de gas SF que las galaxias tienen en las regiones externas, resultando ruidosa la determinación de $\alpha$. Por lo tanto, no podemos afirmar que la relación entre $\alpha$ y $\dot{M}_{\text {accr }}$ a $1<r / r_{50}<5$ sea realmente bivaluada, pero sí podemos asegurar que las galaxias presentan perfiles ligeramente empinados en las regiones externas. Una posibilidad al aplanamiento encontrado en los RMPs a $r / r_{50}>1$ es que la metalicidad del gas del ISM alcance la metalicidad del halo de gas, que estaría actuando como piso de la metalicidad total. Para confirmar esto, debería hacerse un análisis más detallado de la distribución del gas.

Trabajos teóricos previos han encontrado que las fusiones de galaxias y los encuentros cercanos tienen un efecto significativo en el patrón del enriquecimiento químico, de manera que el gas que cae hacia el disco de la galaxia producto de la interacción diluye la metalicidad en las regiones centrales de ésta (Montuori et al., 2010). Observaciones de galaxias a $z \approx 0$ ultra luminosas en infrarrojo sugieren tendencias similares (Rupke et al., 2008), al igual que trabajos en donde se estudian pares interactuantes de galaxias (Rosa et al., 2014). Exploramos esta posibilidad al analizar las pendiente de galaxias que sufrieron fusiones mayores o menores desde la salida previa a la analizada (es decir, galaxias que tuvieron más de un progenitor de $M_{\star} \gtrsim 10^{8} \mathrm{M}_{\odot}$ ). Encontramos que sólo el $10 \%$ de nuestra muestra ha experimentado un evento de fusión de estas características, y que la pendiente $\alpha$ en las regiones interna y externa son muy similares a la muestra completa. Entonces, no encontramos evidencia de que las fusiones afecten los RMPs de las galaxias de manera significativa (al menos a $z \lesssim 1$ ). Esto se debe en parte a que la mayoría de las fusiones de galaxias a $z \lesssim 0.5$ en EAGLE tienen baja fracción de gas (cocientes de masa de gas sobre masa estelar $\lesssim 0.1$, ver la figura 2 en Lagos et al. $2018 b$ ) y así presentan un efecto reducido en los perfiles de metalicidad del gas. Estos objetos resultan diferentes de los estudiados en Rupke et al. (2008), que fueron seleccionados para ser galaxias con alta formación estelar, y diferentes de las simulaciones de Montuori et al. (2010), las cuales generan fusiones ricas en gas. Por lo tanto, no es sorprendente que encontremos efectos más débiles de las fusiones de galaxias.

Dada la fuerte (anti-)correlación entre $\alpha$ y $\dot{M}_{\text {accr }}$ a $r / r_{50}<1$, analizamos ésta en detalle a continuación. De ahora en más, a menos que se estipule lo contrario, nos enfocaremos solamente en la región interna del RMP.

\subsubsection{La relación entre RMP y masa estelar}

La Figura 4.3 muestra la pendiente interna del RMP, $\alpha$, como función de la masa estelar. Ambas simulaciones exhiben una anti-correlación de manera que pendientes más negativas están asociadas 
con galaxias más masivas. Esto podría parecer contradictorio a primera vista con los resultados de Tissera et al. (2019), quienes también usaron EAGLE, pero la principal diferencia es que aquí se obtiene $\alpha$ dentro de $r_{50}$, mientras que Tissera et al. (2019) obtienen sus pendientes entre $0.5 \times r_{50}$ y $2 \times$ $r_{50}$. Como resultado, los RMPs en Tissera et al. (2019) son levemente más planos que los calculados para $r / r_{50}<1$. Entendemos que no hay otros resultados en la literatura con una pendiente del RMP calculada usando el corte que adoptamos aquí. Como consecuencia, no haremos comparaciones de nuestros resultados con hallazgos observacionales o teóricos debido a la falta de consistencia (ver Apéndice D). Sin embargo, incentivamos a otros trabajos, especialmente observacionales, a usar el corte de radio propuesto aquí.
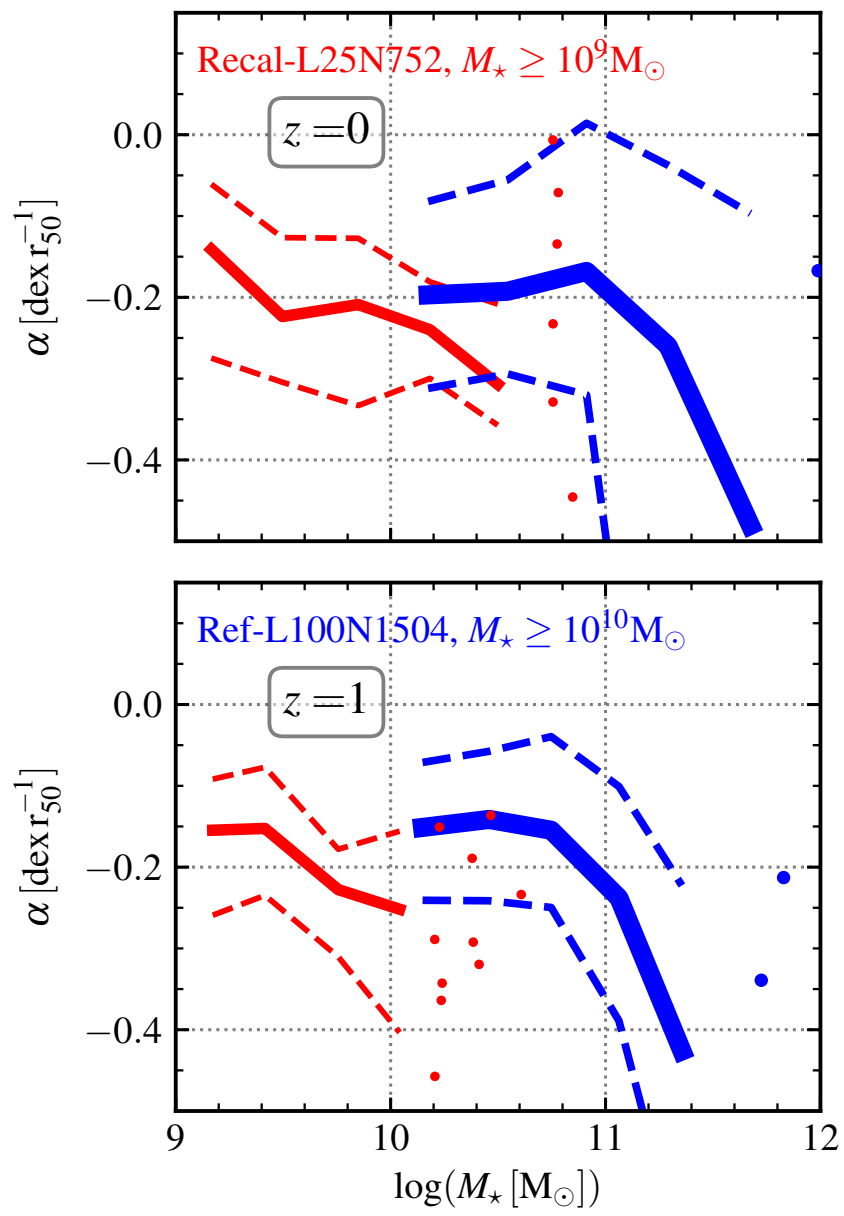

Figura 4.3: Valores medios de la relación de la pendiente interna del RMP, $\alpha$, como función de la masa estelar, $M_{\star}$. Los paneles superior e inferior muestran galaxias a $z=0$ y $z=1$, respectivamente. La simulación RecalL25N752 es mostrada con líneas rojas finas, mientras que la Ref-L100N1504 se muestra con líneas azules gruesas. En todos los casos, las líneas a trazos denotan los percentiles 16 - 84, y aquellas galaxias individuales donde los intervalos tienen $<10$ objetos se muestran con círculos.

Las dos simulaciones analizadas aquí, Recal-L25N752 y Ref-L100N1504, se superponen en una región de masa estelar en la cual se encuentran diferencias entre sí, sugiriendo que la masa estelar podría no ser una propiedad principal en la determinación de $\alpha$. Esto no resulta necesariamente sorprendente, dado que los ambientes de las galaxias con masa estelar de $\approx 10^{10} \mathrm{M}_{\odot}$ en Recal-L25N752 no son los mismos que en la simulación Ref-L100N1504. Furlong et al. (2015) mostró que las SFRs de las galaxias con masas de $10^{9}-10^{10} \mathrm{M}_{\odot}$ son mayores en Recal-L25N752 que en Ref-L100N1504, 
mientras se vuelve más similares para $M_{\star}>10^{10} \mathrm{M}_{\odot}$. Esto nos dice que la $\dot{M}_{\text {accr }}$ es mayor en galaxias de masa estelar $\approx 10^{10} \mathrm{M}_{\odot}$ en Recal-L25N752 que en Ref-L100N1504 (ver Apéndice E para detalles de la relación $\dot{M}_{\text {accr }}-M_{\star}$ ). A continuación demostramos que la $\dot{M}_{\text {accr }}$ es ciertamente la principal modificadora de la pendiente $\alpha$ y que la diferencia vista en la Figura 4.3 resulta por las diferentes $\dot{M}_{\text {accr }}$ que tienen las galaxias de $\approx 10^{10} \mathrm{M}_{\odot}$ en Recal-L25N752 y Ref-L100N1504. Esto no significa que la masa estelar no juegue un rol en la formación de los RMP, sino que los cambios más significativos de éstos se deben a la $\dot{M}_{\text {accr }}$.
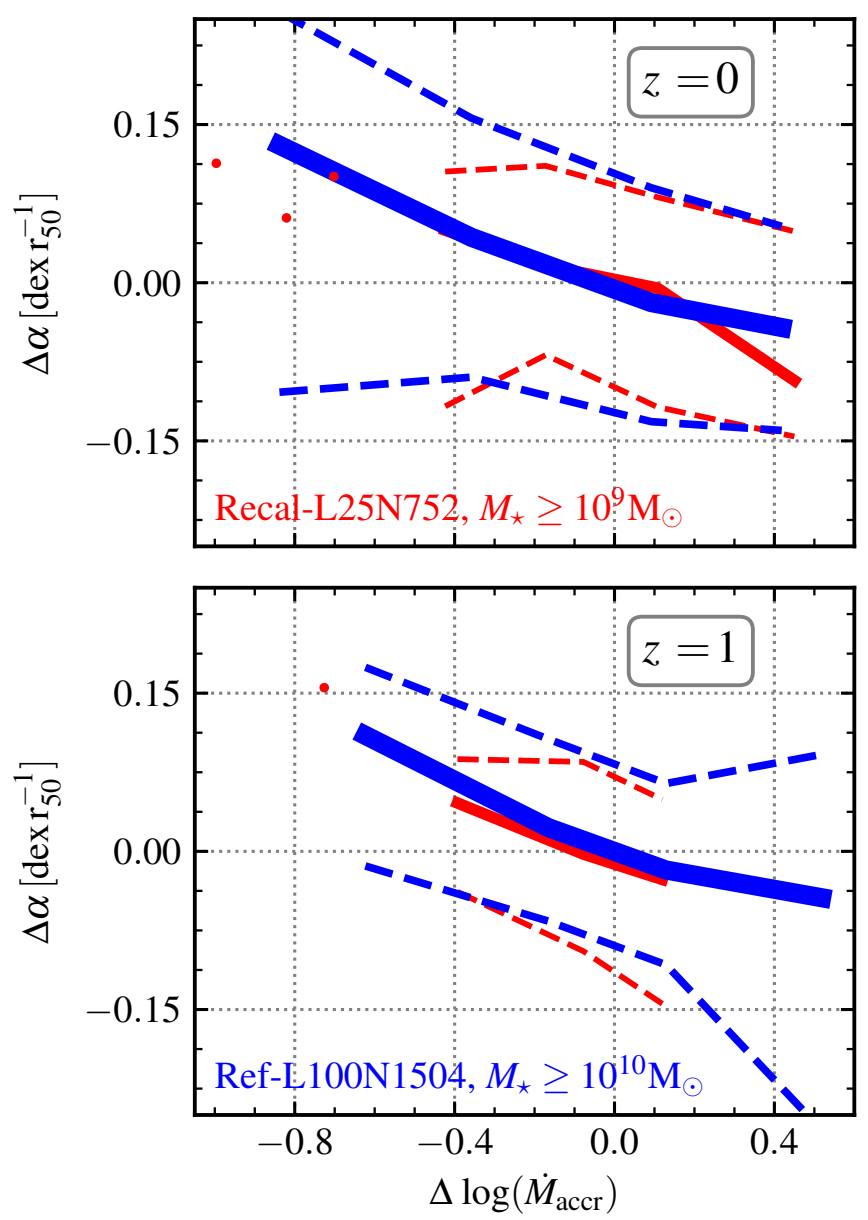

Figura 4.4: Residuos de la relación $\alpha-M_{\star}$ como función de los residuos de la relación $\dot{M}_{\text {accr }}-M_{\star}$, con $\alpha$ siendo la pendiente interna del RMP $\left(r / r_{50} \leq 1\right)$. Paneles superior e inferior muestran galaxias a $z=0$ y $z=$ 1 , respectivamente. Los colores rojo y azul representan las simulaciones Recal-L25N752 y Ref-L100N1504, respectivamente. Los percentiles 16 - 84 están denotados como líneas a trazos, y las líneas sólidas representan valores medios. Mostramos galaxias individuales donde los intervalos tienen $<10$ objetos (círculos rojos). Habiendo eliminado la dependencia con la $M_{\star}$, aún vemos una fuerte anti-correlación entre $\alpha$ y $\dot{M}_{\text {accr }}$, indicando ser independiente de la $M_{\star}$. Ambas simulaciones están en buen acuerdo entre sí. Ref-L100N1504 cubre un amplio rango de $\dot{M}_{\text {accr }}$ debido a que tiene un mayor volumen cosmológico en comparación con Recal-L25N752.

Cuantificamos qué propiedad, $\dot{M}_{\text {accr }}$ o masa estelar, muestra una correlación más fuerte con la pendiente interna $\alpha$, a través del rango del coeficiente de correlación de Spearman $\left(\mathrm{R}_{\mathrm{s}}\right)$, encontrando que $\dot{M}_{\text {accr }}$ devuelve valores absolutos que son similares (Recal-L25N752) o mayores (Ref-L100N1504, encima de $\approx 0.1$ ) que aquéllos obtenidos con la masa estelar a todo redshift. Por lo tanto, en términos de dispersión, $\alpha$ se encuentra mejor correlacionada con $\dot{M}_{\text {accr }}$ que con la masa estelar. Debido a esto, y a la relación entre la masa estelar y la $\dot{M}_{\text {accr }}$, mostramos en la Figura 4.4 los residuos de la rela- 
ción $\alpha-M_{\star}(\Delta \alpha)$ como función de los residuos de la relación $\dot{M}_{\text {accr }}-M_{\star}\left(\Delta \dot{M}_{\text {accr }}\right)$. Definimos estos residuos de la siguiente manera

$$
\Delta X=X-\operatorname{med}(X),
$$

donde $X$ es la propiedad de interés de una galaxia, y $\operatorname{med}(X)$ es el valor medio a la masa estelar de la galaxia.

Al realizar este cálculo, eliminamos la dependencia con la masa estelar de ambos ejes. Encontramos que la anti-correlación entre $\Delta \alpha$ y $\Delta \dot{M}_{\text {accr }}$ permanece. Concluimos que, al menos para las regiones internas, las galaxias a masa fija que tienen mayor $\dot{M}_{\text {accr }}$ también presentan RMPs más inclinados (con pendiente negativa). Aunque hay una expectativa de que valores mayores de $\dot{M}_{\text {accr }}$ puedan generar cambios en el RMP, a nuestro entender es la primera vez que estos resultados son cuantificados en simulaciones cosmológicas. También notamos que los residuos en la Figura 4.4 no muestran diferencias entre las simulaciones estándar y de alta resolución de EAGLE, sugiriendo que las diferencias encontradas en la Figura 4.2 son debidas a diferencias en sus distribuciones de masa.

\subsubsection{La relación entre SFR, $\dot{M}_{\text {accr }}$ y RMP}

Observacionalmente, la estimación de la tasa de acreción de gas es bastante complicada debido a que se espera que el gas acretado sea de baja densidad y, así, apenas visible (ejemplo Fox \& Davé, 2017). Además, también se espera que este gas entrante tenga una cinemática relativamente lenta (comparado con el gas que está siendo eyectado), resultando un desafío separarlo espectroscópicamente de la dispersión de velocidades intrínseca del ISM (Bouché, 2017). Por otro lado, la SFR integrada de una galaxia es mucho más fácil de inferir y se espera que esté estrechamente correlacionada con la tasa de acreción de gas. Esto último es la base de los "modelos de equilibrio" de la evolución de galaxias. En estos modelos, la tasa de acreción de gas es perfectamente balanceada por la combinación de la SFR y las tasas de eyección de material, y $\dot{M}_{\text {accr }}$ puede entonces ser inferida a través de estos. De lo anterior se desprende que la $\dot{M}_{\text {accr }}$ está linealmente correlacionada con la SFR (ejemplos, Davé et al. 2012; Lilly et al. 2013), modulada por el parámetro de carga de masa (el cociente entre la tasa de eyección y la SFR). Como consecuencia, se espera que las correlaciones discutidas en la Sección 4.5.1 se extiendan a las SFRs de las galaxias, es decir, que la pendiente del RMP $\alpha$ decrezca al aumentar la SFR.

Una correlación entre $\alpha$ y la SFR de las galaxias puede tener otras interpretaciones además de la provista por los "modelos de equilibrio". Por ejemplo, es posible que el proceso principal de controlar a $\alpha$ sea el enriquecimiento químico del ISM debido a las recientes estrellas formadas. En este caso, es fácil imaginar que la SFR podría ser una propiedad más fundamental que causa el cambio en la pendiente $\alpha$ en lugar de la tasa de acreción de gas. Este resultado es interesante dado que se encuentra aún en debate si hay una correlación positiva, negativa o incluso nula entre $\alpha$ y la SFR en galaxias (ver la Sección 1.2.2).

En ambos escenarios es probable como resultado una anti-correlación entre $\alpha$ y la SFR, pero en el primer caso se espera que la dispersión sea mayor que en la encontrada relación $\alpha-\dot{M}_{\text {accr }}$, mientras que en el segundo caso el resultado opuesto sería más natural. Para dilucidar la situación más probable entre estos dos casos, estudiamos las mismas correlaciones que en la Figura 4.2 y 4.4 (para regiones internas), pero en función de la SFR de las galaxias.

Antes de proceder con el análisis planteado, verificamos la existencia de una correlación ajustada entre $\dot{M}_{\text {accr }}$ y SFR en la Figura 4.5. En acuerdo con la previsión de los modelos de equilibrio descritos anteriormente, encontramos una fuerte correlación entre $\dot{M}_{\text {accr }}$ y SFR a todo redshift estudiado, con una dispersión de $1 \sigma$ de $\lesssim 0.5$ dex para las simulación Ref-L100N1504 y de $\lesssim 0.1$ dex para Recal-L25N752. Esta dispersión decrece significativamente con la SFR, desde $\approx 0.5$ dex para SFR $\lesssim 0.45 \mathrm{M}_{\odot} \mathrm{yr}^{-1}$ hasta $\approx 0.1$ dex a SFR $\gtrsim 6 \mathrm{M}_{\odot} \mathrm{yr}^{-1}$. Aunque sí esperamos una correlación en general entre SFR y $\dot{M}_{\text {accr }}$, la dispersión aquí hallada puede ser artificialmente pequeña considerando nuestra 
definición de gas acretado, la cual se basa en acreción de gas que lleva a la formación estelar (ver la Sección 4.2 para detalles). Es interesante notar que la relación media predicha evoluciona débilmente con el redshift, al menos hasta $z \leq 1$, con diferencias de $\lesssim 0.3$ dex entre $z=1$ y 0 a SFR fija.
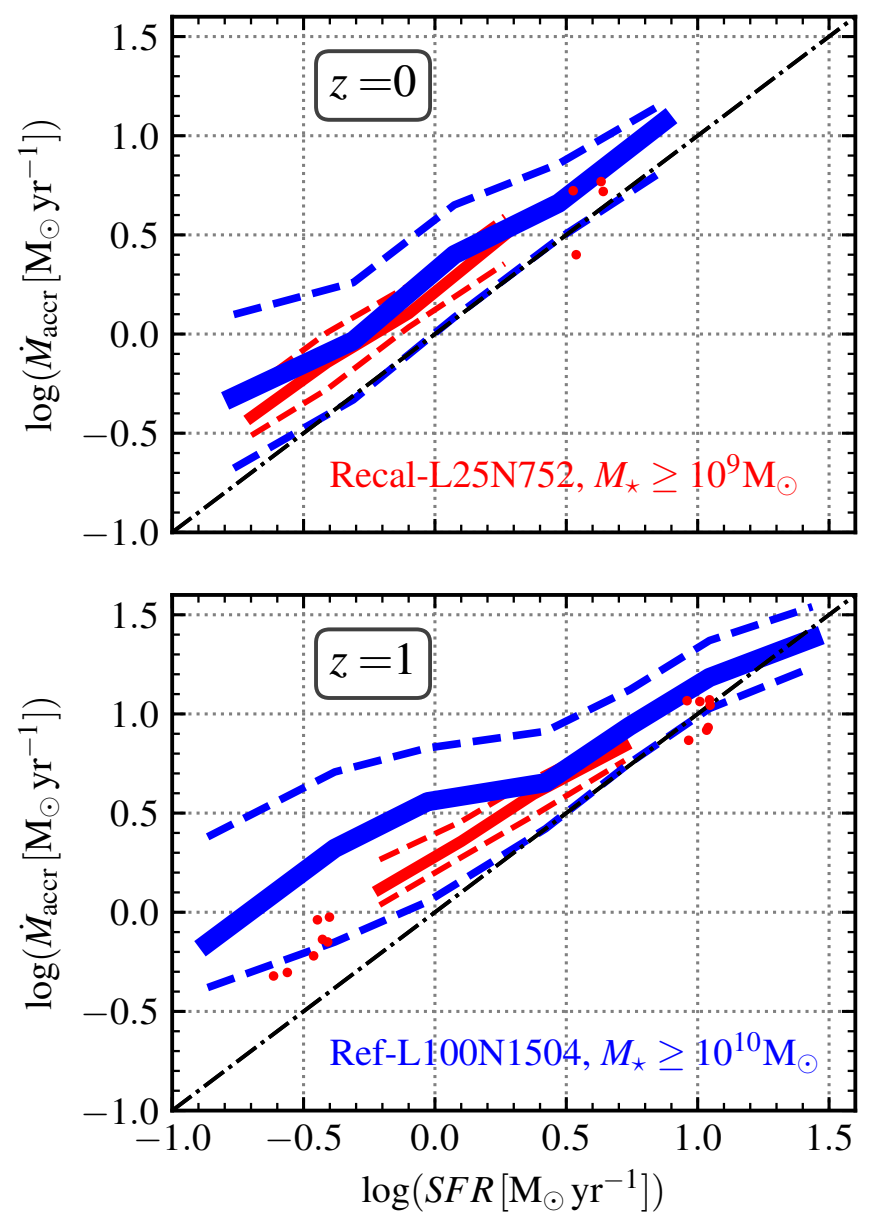

Figura 4.5: $\dot{M}_{\text {accr }}$ como función de la SFR de galaxias en las simulaciones Recal-L25N752 (rojo) y RefL100N1504 (azul). Paneles superior e inferior muestran las galaxias de EAGLE a $z=0$ y $z=1$, respectivamente. Los percentiles $16-84$ son mostrados con líneas a trazos. Mostramos galaxias individuales donde los intervalos tienen $<10$ objetos como círculos. Las líneas punteadas a trazos muestran la relación identidad 1:1, a modo de comparación con las pendientes de las relaciones simuladas. Recordamos que las galaxias de Recal-L25N752 fueron seleccionadas para tener $M_{\star} \geq 10^{9} \mathrm{M}_{\odot}$, mientras que las galaxias de Ref-L100N1504 fueron seleccionadas para tener $M_{\star} \geq 10^{10} \mathrm{M}_{\odot}$. Ambas simulaciones exhiben una correlación positiva y ajustada entre la $\dot{M}_{\text {accr }}$ y la SFR. Además, a SFR fija, las galaxias muestran un débil decrecimiento de su $\dot{M}_{\text {accr }}$ a $\approx 0.2-0.3$ dex desde $z=1$ a 0 (sólo apreciable para galaxias con $\mathrm{SFR}<10^{0.5} \mathrm{M}_{\odot} \mathrm{yr}^{-1}$ ).

Los paneles izquierdos de la Figura 4.6 muestran la pendiente del RMP en la región interna $\left(r / r_{50}<1\right)$ como función de la SFR para $z=0$ y 1 . Esta relación tiene una tendencia y dispersión similares a la hallada para $\dot{M}_{\text {accr }}$ (ver Figura 4.2). Cuantificamos las correlaciones siguiendo el mismo procedimiento aplicado en la Sección 4.5.2, encontrando que la relación $\alpha-\dot{M}_{\text {accr }}$ tiene un $\mathrm{R}_{\mathrm{s}}$ similar a (Recal-L25N752) o superior por $\sim 0.1$ (Ref-L100N1504) que la relación $\alpha$-SFR (nuevamente, $\dot{M}_{\text {accr }}$ alcanza valores de Rs más negativos que -0.3), sugiriendo que la correlación más fundamental es con $\dot{M}_{\text {accr. }}$ Tissera et al. (2019) mostraron que las galaxias de EAGLE con pendientes más negativas tienden a tener una fracción de sus estrellas recientemente formadas más grandes, consistente con el hecho que ha habido más acreción de gas que llevó a esa actividad de formación estelar, y con la clara 
anti-correlación que encontramos aquí entre $\alpha$ y la SFR. Sin embargo, recordamos que Tissera et al. (2019) midieron pendientes únicas de todo el RMP en lugar de separar en distintas regiones del disco.
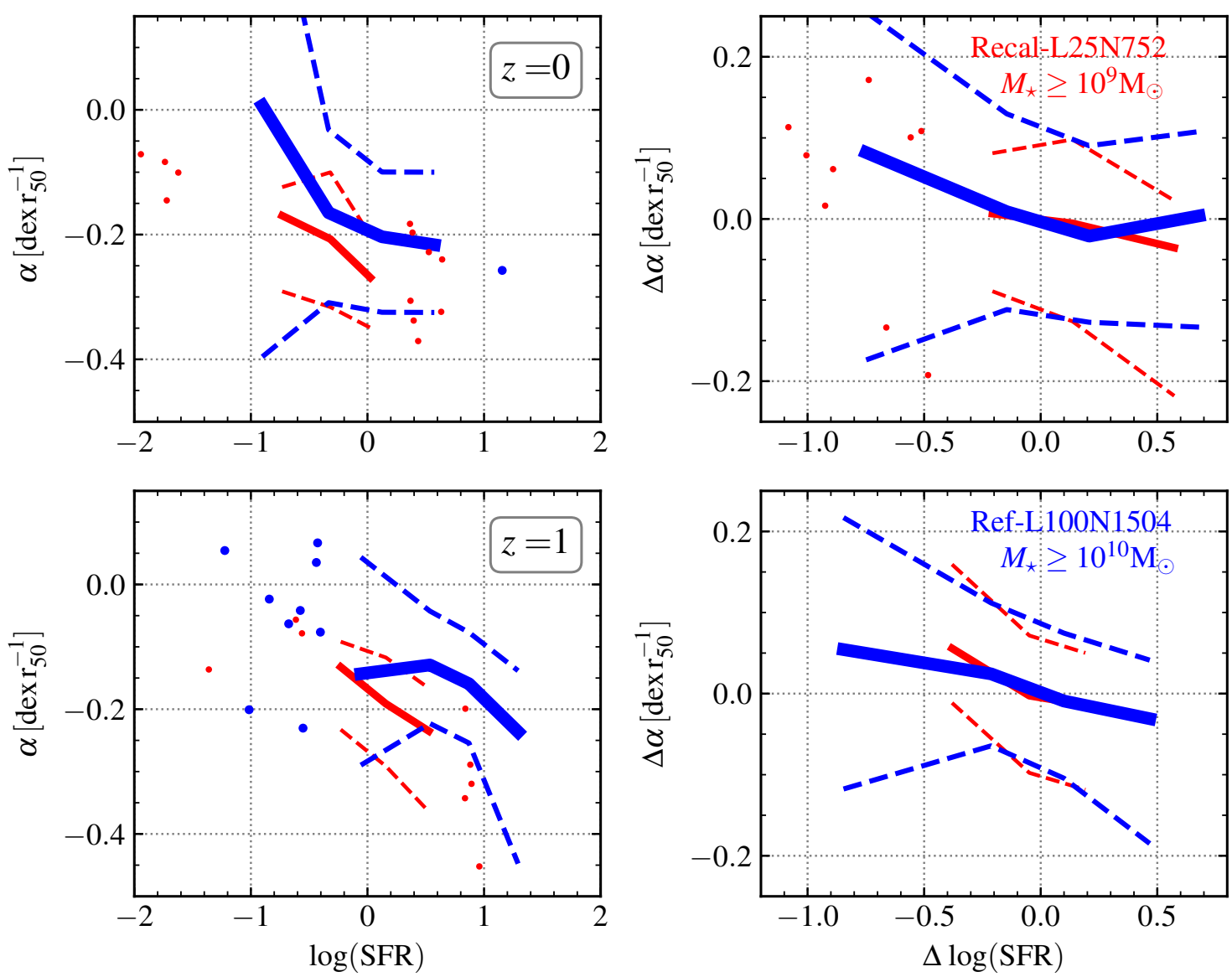

Figura 4.6: Izquierda: Las líneas sólidas muestran los valores medios de la pendiente interna del RMP $\left(r / r_{50} \leq\right.$ 1) como función de la SFR a redshift $z=0$ (panel superior) y a $z=1$ (panel inferior), para las simulaciones Recal-L25N752 (rojo) y Ref-L100N1504 (azul). Una anti-correlación similar es obtenida para $\alpha-\dot{M}_{\text {accr }}$ (panel izquierdo de la Figura 4.5.1). Derecha: Las líneas sólidas muestran los valores medios de los residuos de la relación entre la pendiente interna del RMP y la $M_{\star}$, como función de los residuos de la SFR y la $M_{\star}$. Los redshifts analizados se muestran en la Figura. Una vez que se elimina la dependencia con la $M_{\star}$ (paneles derechos), la anti-correlación es más débil que la hallada entre $\Delta \alpha$ y $\Delta \log \left(\dot{M}_{\text {accr }}\right)$ mostrada en la Figura 4.4. Esto sugiere que la anti-correlación entre la pendiente del RMP y la SFR es sólo un resultado de la anticorrelación entre la pendiente del RMP y $\dot{M}_{\text {accr }}$. Todos los paneles: los percentiles $16-84$ son denotados con líneas a trazos. Mostramos galaxias individuales donde los intervalos tienen $<10$ objetos (círculos).

De manera similar a lo hecho para $\dot{M}_{\text {accr }}$, removemos la dependencia con la masa estelar al estudiar los residuos entre la relación $\alpha-M_{\star}$ como función de los residuos de la relación SFR $-M_{\star}$ (ver Ecuación 4.3) en los paneles derechos de la Figura 4.6 para $z=0$ y 1. Como se ha explicado en la Sección 4.5.2, los residuos son construidos como la diferencia entre la propiedad estudiada (en este caso, $\alpha$ o SFR) y la media a la masa estelar de la galaxia. De manera similar al caso de la Figura 4.4, al eliminar la dependencia con la masa estelar, ambas simulaciones predicen valores que están en acuerdo entre sí. La simulación Recal-L25N752 exhibe una débil anti-correlación entre $\Delta \alpha$ y $\Delta$ SFR (con un coeficiente de correlación de Spearman $\mathrm{R}_{\mathrm{s}} \approx-0.2$ ), similar a la anti-correlación entre $\Delta \alpha \mathrm{y}$ $\Delta \dot{M}_{\text {accr }}$ (Figura 4.4). Sin embargo, la simulación Ref-L100N1504 muestra una fuerte anti-correlación con $\mathrm{R}_{\mathrm{S}} \approx-0.3$. Estos resultados sugieren que la relación $\alpha-\mathrm{SFR}$ es una consecuencia de otras propie- 
dades involucradas, como la $\dot{M}_{\text {accr }}$ y la masa estelar. Por lo tanto, concluimos que la pendiente interna del RMP es principalmente modelada por la tasa de acreción de gas en lugar que por la SFR.

\subsubsection{La relación entre RMP y la fracción de gas}

En el llamado "modelo de equilibrio", $\dot{M}_{\text {accr }}$ regula el contenido gaseoso, la SFR y la metalicidad de las galaxias. Se espera, entonces, que la fracción de gas de una galaxia sea una trazadora de la acreción de gas (modulada por la escala de tiempo que toma en convertir gas en estrellas y en generar eyecciones). La fracción de gas es atractiva en el sentido que resulta más disponible que la $\dot{M}_{\text {accr }}$, y puede ser relacionada con esta última (aunque bajo ciertas suposiciones). Por lo tanto, se espera que una simulación de formación de galaxias en general capture la naturaleza de las galaxias $\mathrm{SF}$, produciendo una relación entre la metalicidad del gas de la galaxia y su fracción de gas. Aquí exploramos la relación entre el RMP y la fracción de gas de las galaxias.
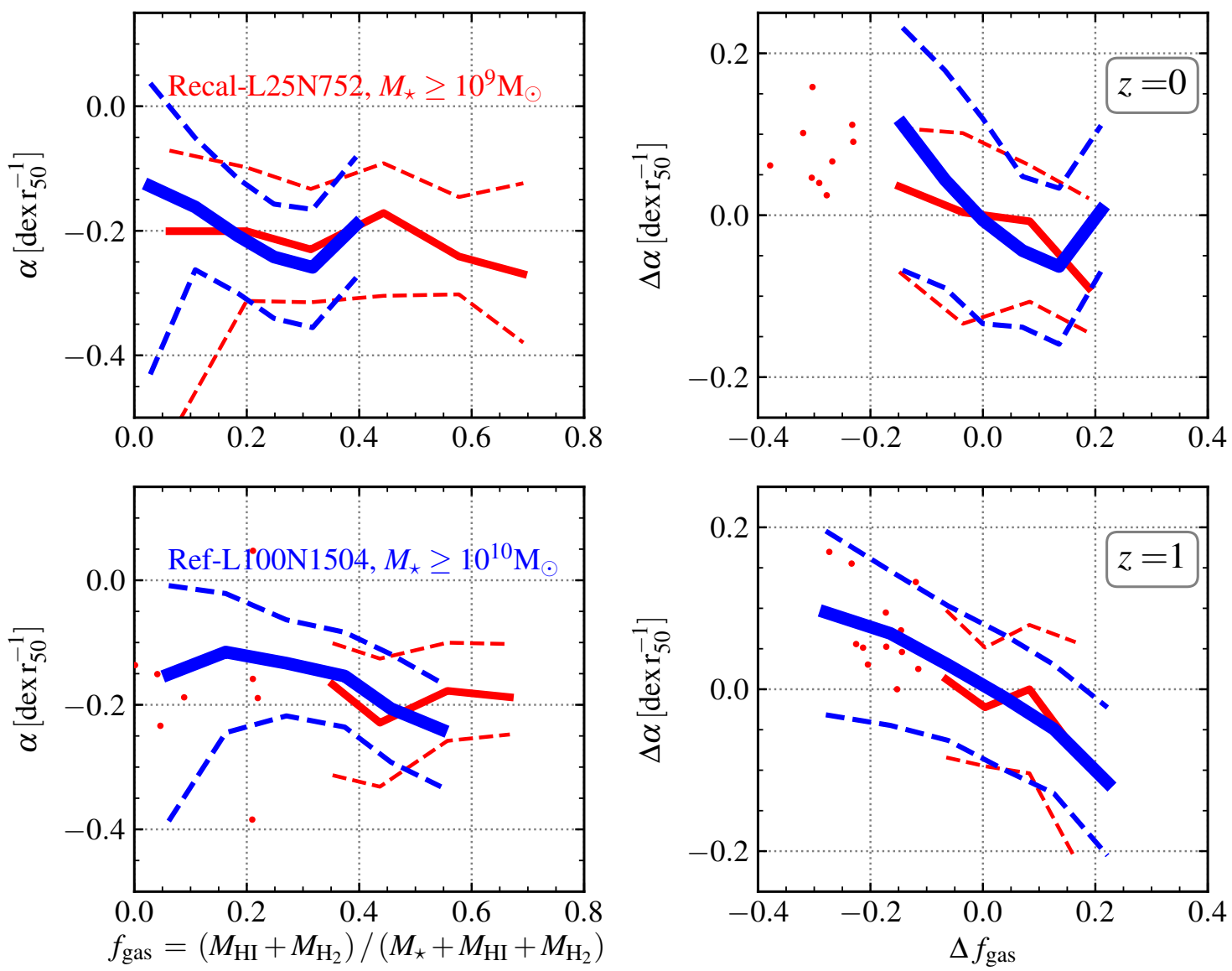

Figura 4.7: Izquierda: Las líneas sólidas muestran los valores medios de la pendiente interna del RMP $\left(r / r_{50} \leq\right.$ 1) como función de la fracción de gas neutro a redshift $z=0$ (panel superior) y a $z=1$ (panel inferior), para las simulaciones Recal-L25N752 (rojo) y Ref-L100N1504 (azul). Una anti-correlación similar es obtenida para $\alpha-\dot{M}_{\text {accr }}$ (panel izquierdo de la Figura 4.5.1). Derecha: Las líneas sólidas muestran los valores medios de los residuos de la relación entre la pendiente interna del RMP y la $M_{\star}$, como función de los residuos de la $f_{\text {gas }}$ y la $M_{\star}$. Los redshifts analizados se muestran en la Figura. Como se mostró en la Figura 4.4, la pendiente interna del RMP exhibe una anti-correlación con $f_{\text {gas }}$ incluso luego de eliminar la dependencia con la $M_{\star}$. Todos $l o s$ paneles: los percentiles 16 - 84 son denotados con líneas a trazos. Mostramos galaxias individuales donde los intervalos tienen $<10$ objetos (círculos rojos). 
Utilizamos las mediciones de fracción de gas neutro de Lagos et al. (2016), definido como la masa de gas neutro dentro de $30 \mathrm{kpc}, M_{\mathrm{HI}}+M_{\mathrm{H}_{2}}$, sobre la suma del anterior y la masa bariónica en la misma apertura, $M_{\star}+M_{\mathrm{HI}}+M_{\mathrm{H}_{2}}$. La masa de gas neutro se encuentra al aplicar la separación entre el gas ionizado al neutro en el post-procesado siguiendo a Rahmati et al. (2013a, ver Lagos et al. 2015 para más detalles sobre la partición en fases de partículas de gas en el subgrillado).

La columna izquierda de la Figura 4.7 muestra la pendiente del RMP a $r / r_{50}<1$ como función de la fracción de gas neutro, $f_{\text {gas }}$, a $z=0$ y 1 para las simulaciones Recal-L25N752 (rojo) y Ref-L100N1504 (azul). Si primero analizamos la simulación Ref-L100N1504, y para todo redshift estudiado, vemos que hay una tendencia de que el RMP decrezca con el radio de manera más inclinada a medida que la fracción de gas incrementa. Esto se debe a que las fracciones de gas altas están asociadas con $\dot{M}_{\text {accr }}$ mayores. Sin embargo, en la simulación Recal-L25N752 vemos un comportamiento diferente, ya que o bien no se observa cambios en la pendiente a medida que aumenta la fracción de gas o bien hay intervalos en donde se invierte la relación. Esta diferencia resulta porque la fracción de gas está fuertemente anti-correlacionada con la masa estelar (ver la figura 1 de Lagos et al. 2016). Por lo tanto, es necesario remover la dependencia con la masa estelar para develar una posible correlación entre el RMP y la fracción de gas.

La columna derecha de la Figura 4.7 muestra la relación entre los residuos de la pendiente del RMP $(\Delta \alpha)$ y los residuos de la fracción de gas $\left(\Delta f_{\text {gas }}\right)$, siguiendo la definición de la Ecuación 4.3 (Sección 4.5.2). La correlación se muestra para $z=0$ (panel superior) y $z=1$ (panel inferior). Es interesante notar que una vez que la dependencia con la masa estelar es removida, una fuerte anti-correlación es encontrada (a excepción tal vez de la simulación Ref-L100N1504 en el panel superior derecho que muestra una correlación positiva para el intervalo de $\Delta f \sim 0.2$ ), lo que recuerda a la Figura 4.4. Esto podría estar relacionado con el hecho de que todas las propiedades de galaxias mencionadas aquí ( $\dot{M}_{\text {accr }}$, SFR y $f_{\text {gas }}$ ) modulan, con diferente grado, la pendiente del RMP a masa estelar fija.

Computamos el coeficiente de correlación de Spearman $\mathrm{R}_{\mathrm{s}}$ para la relación $\Delta \alpha-\Delta f_{\text {gas }}\left(\mathrm{R}_{\mathrm{s} \text {,gas }}\right) \mathrm{y}$ lo comparamos con la obtenida para la relación $\Delta \alpha-\Delta \dot{M}_{\mathrm{accr}}\left(\mathrm{R}_{\mathrm{s}, \text { accr }}\right)$. Ambos $\mathrm{R}_{\mathrm{s}}$ son similares para las simulaciones Ref-L100N1504 y Recal-L25N752, con valores de $\approx-0.2$ y $\approx-0.3$, respectivamente. Es importante mencionar, sin embargo, que los valores absolutos de $\mathrm{R}_{\mathrm{s}}$ indican que la relación $\alpha-\dot{M}_{\text {accr }}$ es más fuerte que la relación $\alpha-f_{\text {gas }}$ a todo redshift considerado.

Es curioso que la relación de la pendiente del RMP con $f_{\text {gas }}$ cambie tanto yendo de los paneles izquierdos a derechos de la Figura 4.7, comparado con lo que sucede para el caso de la $\dot{M}_{\text {accr }}$ (Figuras 4.2 y 4.4) y de la SFR (Figura 4.6). Estas grandes discrepancias resultan del cambio de la correlación entre $\dot{M}_{\text {accr }}$ y la masa de gas $\left(M_{\mathrm{HI}}+M_{\mathrm{H}_{2}}\right)$ con la masa estelar. Esta relación es positiva a baja masa estelar, pero se aplana o incluso se invierte a medida que la masa estelar crece. Por lo tanto, a baja masa estelar $\left(M_{\star} \lesssim 10^{10} \mathrm{M}_{\odot}\right)$, la acreción de gas mayormente incrementa la fracción de gas de la galaxia (que es el caso para las galaxias enanas), mientras que a altas masa estelares $\left(M_{\star} \gtrsim 10^{10.5} \mathrm{M}_{\odot}\right)$, la acreción de gas desencadenaría el feedback de AGN que luego reduce la fracción de gas. Entonces, a pesar de que la relación entre la pendiente del RMP y la $f_{\text {gas }}$ provea de restricciones importantes sobre el efecto de acreción de gas, no resulta una relación simple ni directa como lo es la que obtuvimos entre la pendiente del RMP y $\dot{M}_{\text {accr }}$. Ver el Apéndice F para la descripción de los resultados cuando se considera solamente la componente de gas molecular $\left(M_{\mathrm{H}_{2}}\right)$ para la fracción de gas, lo cual puede ser de utilidad dado que el gas atómico $\left(M_{\mathrm{HI}}\right)$ no es actualmente accesible en observaciones a $z \gtrsim 0.4$.

\subsection{Discusión sobre el impacto de la $\dot{M}_{\text {accr }}$ en los RMP}

Hemos mostrado en Secciones previas (4.5.1 y 4.5.3) que la acreción de gas juega un papel importante en la determinación de la pendiente interna de los RMPs. También, concluimos que otras 
propiedades de galaxias, como la SFR, la masa estelar y la fracción de gas, tienen un rol secundario en el proceso de modificar la pendiente, y es altamente posible que tal proceso de modificación sea el producto de la correlación de estas propiedades con la $\dot{M}_{\text {accr }}$. Para tener una mejor visualización del efecto de la acreción de gas en los RMPs, mostramos en la Figura 4.8 el RMP de las galaxias en intervalos de masa estelar y $\dot{M}_{\text {accr }}$, para la simulación Ref-L100N1504, a $z=0$ (izquierda) y $z=1$ (derecha). Los paneles superiores muestran galaxias con rangos de masa de $M_{\star} / \mathrm{M}_{\odot} \in\left[10^{10}, 10^{10.3}\right]$, mientras que los paneles inferiores muestran galaxias de masas estelares de $M_{\star} / \mathrm{M}_{\odot} \in\left[10^{10.3}, 10^{11}\right]$. Clasificamos los valores de $\dot{M}_{\text {accr }}$ y presentamos las medias de los RMPs de galaxias en tres percentiles de $\dot{M}_{\text {accr: }}$ el $25 \%$ inferior, entre el $40 \%-60 \%$ y el $25 \%$ superior. Esto nos permite comparar galaxias de manera independiente de la evolución general de la $\dot{M}_{\text {accr }}$.
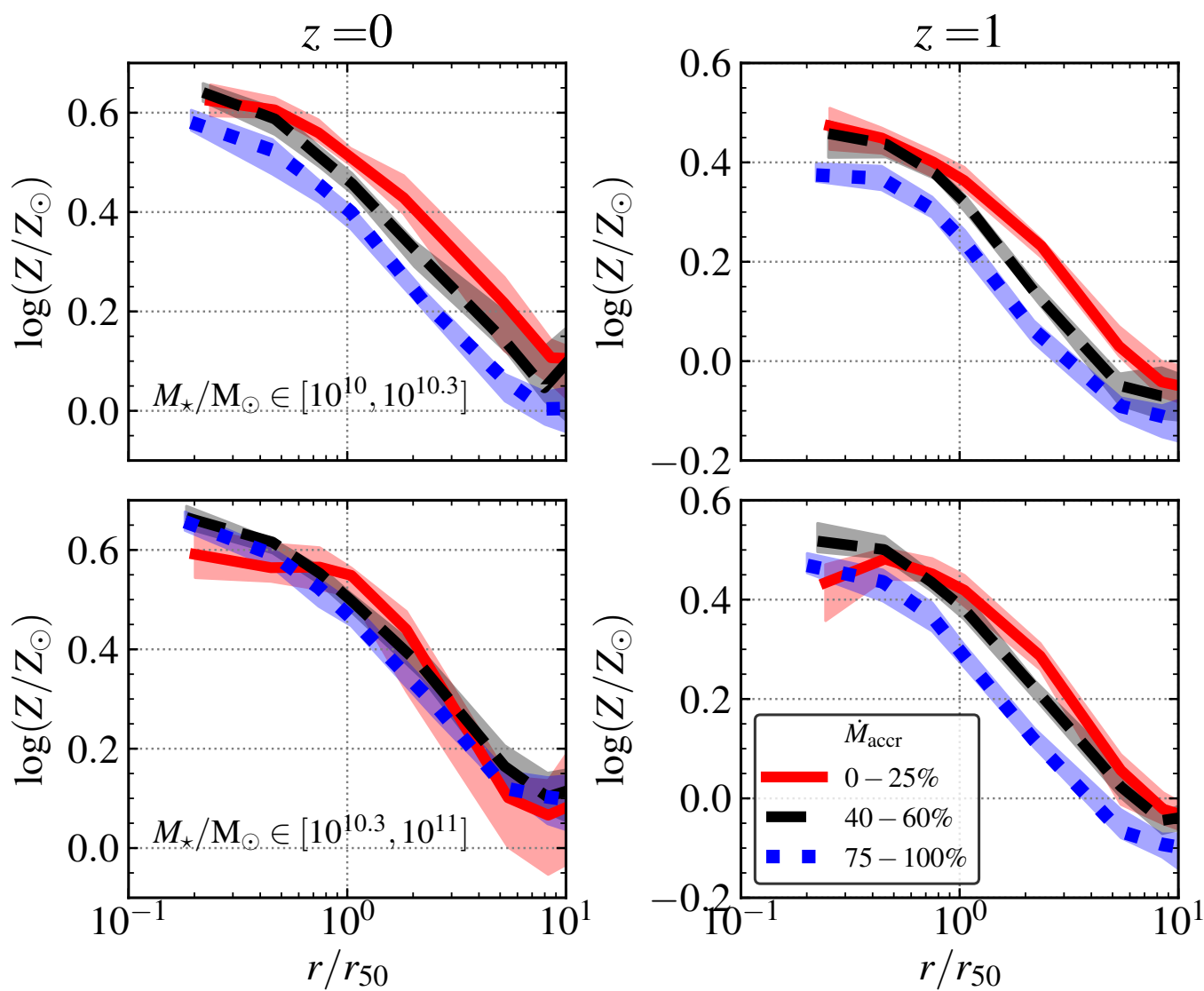

Figura 4.8: RMP a $z=0$ (paneles izquierdos) y a $z=1$ (paneles derechos) para galaxias en la simulación RefL100N1504. Los paneles superiores muestran galaxias en el rango de masa de $M_{\star} / \mathrm{M}_{\odot} \in\left[10^{10}, 10^{10.3}\right]$, mientras que los paneles inferiores muestran el rango $M_{\star} / \mathrm{M}_{\odot} \in\left[10^{10.3}, 10^{11}\right]$. Los diferentes colores representan los cortes en $\dot{M}_{\text {accr }}$, como se muestra en los paneles. Las líneas y regiones sombreadas muestran los valores medios de los RMPs y sus percentiles 16 - 84, respectivamente. Galaxias con mayor $\dot{M}_{\text {accr }}$ (líneas a trazos) muestran una pendiente más empinada a $r<r_{50}$. Dado que el softening de la simulación Ref-L100N1504 es de 0.7 kpc y encontramos valores promedios de $r_{50} \approx 4.22 \mathrm{kpc} \mathrm{a} z=0$ y de $r_{50} \approx 3.11 \mathrm{kpc} \mathrm{a} z=1$, los perfiles resultan bien resueltos para $r / r_{50} \leq 0.17$ y $r / r_{50} \leq 0.23$, respectivamente. Es interesante notar que, a $M_{\star}$ fija, hay un desplazamiento vertical de la normalización de la metalicidad de acuerdo a los diferentes valores tomados por la $\dot{M}_{\text {accr }}$, como se discute en la Sección 4.4.

Las galaxias con mayor $\dot{M}_{\text {accr }}$ muestran pendientes internas más inclinadas, independientemente de su masa estelar y redshift. En el rango de masa estelar de $10^{10}-10^{10.3} \mathrm{M}_{\odot}$ a $z=0$ (panel superior 
izquierdo en la Figura 4.8), las galaxias tienen metalicidades de gas similares en el centro. Sin embargo, las galaxias con menor $\dot{M}_{\text {accr }}$ tienen RMPs con centros más planos que se extienden bastante en radio $\left(r \approx 0.5 \times r_{50}\right)$, mientras que las galaxias con mayor $\dot{M}_{\text {accr }}$ no muestran un núcleo en sus RMPs y caen abruptamente. Este comportamiento no es universal (el panel superior derecho no muestra tal caída abrupta). Notar que a radios grandes $\left(r \approx 10 \times r_{50}\right)$ las galaxias exhiben un aplanamiento en sus RMPs, probablemente debido a que el ISM alcanza los valores de metalicidad del CGM. Esto último sucede a radios sistemáticamente más pequeños para galaxias con mayor $\dot{M}_{\mathrm{accr}}$. En galaxias más masivas (panel inferior izquierdo en la Figura 4.8), el núcleo plano del RMP detectado en galaxias de baja $\dot{M}_{\text {accr }}$ es incluso más prominente, extendiéndose hasta $r \approx r_{50}$, mientras que galaxias con mayores $\dot{M}_{\text {accr }}$ no muestran dicho núcleo. A $z=1$, la principal diferencia es que las metalicidades del gas son en general menores que a $z=0$, y la diferencia en normalización de los RMPs a masa estelar fija para diferentes $\dot{M}_{\text {accr }}$ es mayor.

Curiosamente, los perfiles con los menores valores de $\dot{M}_{\text {accr }}$ en galaxias masivas (es decir, con masas estelares en el rango de $M_{\star} / \mathrm{M}_{\odot} \in\left[10^{10.3}, 10^{11}\right]$ ) muestran una caída en las partes más internas del RMP $\left(r<0.5 \times r_{50}\right)$. Para entender este comportamiento en más detalle, analizamos el cociente entre la masa del agujero negro central y la masa estelar $\left(M_{\mathrm{BH}} / M_{\star}\right)$ de las galaxias en la Figura 4.8. Encontramos que, independientemente del redshift, $M_{\mathrm{BH}} / M_{\star}$ decrece mientras $\dot{M}_{\text {accr }}$ incrementa a masa estelar fija, indicando que la $M_{\mathrm{BH}}$ es más masiva para menores valores de $\dot{M}_{\mathrm{accr}}$. Sumado a esto, encontramos que las galaxias más masivas a $z=1$ del corte del $25 \%$ menor de $\dot{M}_{\text {accr }}$ tienen $M_{\mathrm{BH}} / M_{\star} \approx 0.0007$, lo cual es alrededor del doble de lo encontrado para los otros intervalos de $\dot{M}_{\text {accr }}$ y masa estelar. A $z=0$, la tendencia entre $M_{\mathrm{BH}} / M_{\star}$ y $\dot{M}_{\text {accr }}$ se mantiene, pero con menores diferencias entre los distintos cortes de $\dot{M}_{\text {accr }}$. Esto significa que los BHs en la submuestra a $z=1$ de las galaxias más masivas con más bajo $\dot{M}_{\text {accr }}$ tienen los mayores potenciales para afectar a su galaxia huésped. Se espera, entonces, que esta fuerte actividad de AGN remueva el gas del centro de la galaxia, especialmente los elementos pesados, dado que las partes internas tienden a ser más ricas en metales. Siguiendo los argumentos de Bower et al. (2017), el gas en el centro de estas galaxias forma estrellas y enciende un rápido crecimiento del $\mathrm{BH}$ que produce una eyección que expulsa cantidades significativas de gas y metales de la galaxia antes de que los metales tengan tiempo de mezclarse con el gas circundante. La caída del RMP en galaxias con $M_{\star} \in\left[10^{10.3}, 10^{11}\right] \mathrm{M}_{\odot}$ para el rango menor de $\dot{M}_{\text {accr }}$ a $z=1$ es, entonces, producto de un fuerte feedback de AGN que estas galaxias están atravesando, el cual remueve metales de sus centros antes que estos tengan tiempo de mezclarse con el ISM.

Nuestro análisis también puede dar indicios sobre el lugar donde el gas fue recientemente acretado en la galaxia. Las galaxias con mayor $\dot{M}_{\text {accr }}$ muestran pendientes negativas en las partes internas. Entonces, la acreción de gas parecería estar diluyendo los metales desde las regiones externas a las internas, lo que se traduce en perfiles más inclinados. Sin embargo, para corroborar esto es necesario un estudio de las propiedades localmente resueltas de las galaxias (ver Capítulo 5).

Una consideración importante para interpretar las tendencias observadas antes mencionadas es la forma en que calculamos $\dot{M}_{\text {accr }}$. Debido a que estamos siguiendo específicamente partículas que al tiempo de interés son SF, pero que no lo eran en el paso de tiempo anterior, es natural esperar que nuestras mediciones estén sesgadas hacia acreciones de gas que lleven a la formación estelar y, entonces, lleven a cambios más importantes en los centros de los RMPs (donde la mayoría de las estrellas se forman ${ }^{2}$ ). Con esta técnica, entonces, es más probable que estemos viendo el efecto mayor que la acreción de gas pueda tener en el RMP interno. Sin embargo, nuestra medición de la acreción de gas es un límite inferior, ya que por construcción estamos ignorando las partículas de gas que se unen al ISM y que no están relacionadas con la formación estelar, así como también las partículas que fueron eyectadas entre salidas.

\footnotetext{
${ }^{2}$ Recordamos que esto se realiza para facilitar la comparación con las estimaciones observacionales, las cuales típicamente usan líneas de emisión nebular que trazan las regiones HII.
} 
Otra advertencia importante a tener en cuenta es que estamos promediando radialmente los efectos de la acreción de gas, que no resultan tener necesariamente simetría axial según muestran las simulaciones. Esto podría estar borrando parte del efecto más localizado que la acreción de gas produce, particularmente si es fuertemente filamentaria (ejemplos, Sánchez Almeida et al. 2015; Putman 2017; Kacprzak 2017; Ho et al. 2019).

A pesar de estas limitaciones, nuestro trabajo establece claramente el papel importante de la acreción de gas en moldear los RMPs de las galaxias, el cual fuimos capaces de controlar por otros efectos, como las variaciones de masa estelar y SFR, gracias a la estadística de las simulaciones EAGLE, encontrando que la acreción de gas es la principal responsable de la forma de la pendiente de los RMPs. Notar que las metalicidades, SFRs, y fracciones de gas no fueron usadas en el proceso de calibración en EAGLE y, por tanto, los resultados que presentamos aquí representan verdaderas predicciones de la simulación.

Nuestros resultados establecen algunas correlaciones claras que esperaríamos a masa estelar fija: galaxias con RMPs más inclinados (pendientes más negativas) a $r / r_{50}<1$ tienden a tener una mayor fracción de gas neutro y $\dot{M}_{\text {accr }}$ (siendo la última cantidad más difícil de testear).

Mediciones más profundas del contenido de HI en galaxias que lleguen a densidades columnares bajas, típicas del CGM (Popping et al., 2009), junto con estimaciones de gradientes de metalicidad de relevamientos IFS, son una combinación ideal para testear nuestras predicciones. Instrumentos como el Australian Square Kilometre Array (SKA) Pathfinder (ASKAP Johnston et al., 2008) y el Karoo Array Telescope (MeerKAT Booth et al., 2009), y en el futuro el SKA, permitirán estimar el contenido de HI, mientras que instrumentos como el SAMI (Bryant et al., 2015), MUSE (Carton et al., 2018), entre otros, permitirán realizar mediciones de gradientes de metalicidad. Notar que nuestros resultados no pueden ser fácilmente extrapolados a $z>1$ debido a que se espera que las fusiones de galaxias sean más comunes (como fue mostrado por Qu et al., 2017; Lagos et al., 2018b, para EAGLE) y porque nuestra $\dot{M}_{\text {accr }}$ ignora el gas que viene de las fusiones de galaxias (dado que estamos apuntando a cuantificar la acreción "suave"), lo que puede resultar en una importante falta. Además, se espera que la $\dot{M}_{\text {accr }}$ a $z \gtrsim 1$ sea más colimada y penetre en las galaxias de manera más fácil que a $z \lesssim 1$ (ejemplo Correa et al., 2018). Esto puede tener el efecto de alimentar directamente las regiones centrales de la galaxia en lugar de hacerlo desde afuera hacia adentro, posiblemente llevando a la inversión de los RMPs vistos en observaciones a $z \approx 3-4$ (Cresci et al., 2010; Troncoso et al., 2014).

Recientemente, Patrício et al. (2019) presentaron estimaciones observacionales de RMPs de 3 galaxias sometidas al efecto de lente gravitacional fuerte a $z=0.6,0.8$ y 1 , y reportaron que tenían valores de $\alpha$ más negativos que galaxias a $z=0$. Esto es consistente con nuestros hallazgos, donde galaxias a $z \approx 1$ tienen mayor $\dot{M}_{\text {accr }}$, por lo tanto llevan a $\alpha$ más negativas. Sin embargo, para confirmar esto observacionalmente, una muestra de galaxias mayor es requerida para estudiar el RMP a masa estelar fija a través del tiempo cósmico.

\subsection{Conclusiones sobre el estudio de los RMPs}

Usamos dos de las simulaciones del proyecto EAGLE, la simulación de referencia de gran volumen Ref-L100N1504 y la simulación de alta resolución Recal-L25N752, para estudiar cómo la pendiente de los RMPs de las galaxias, $\alpha$, cambia con la $\dot{M}_{\text {accr }}$ y otras propiedades galácticas. Debido a los límites de resolución de estas simulaciones, nos enfocamos en galaxias con $M_{\star} \geq 10^{10} \mathrm{M}_{\odot}$ para la simulación Ref-L100N1504, y $M_{\star} \geq 10^{9} \mathrm{M}_{\odot}$ para la simulación Recal-L25N752. También limitamos nuestro estudio a galaxias SF centrales y a redshifts $z \leq 1$.

Usamos un método de seguimiento de partículas para encontrar las partículas de gas que están siendo acretadas por las galaxias, incluyendo aquellas partículas que se han convertido en estrellas. Prestamos especial interés a la acreción suave en lugar de la acreción resultante de fusiones de gala- 
xias, y así seleccionamos sólo aquellas partículas de gas que no fueron parte de otra galaxia en una salida de tiempo anterior. Nuestro objetivo es encontrar si la $\dot{M}_{\text {accr }}$ puede ser robustamente definida como principal modificadora del RMP, y luego controlar por diferencias en la masa estelar y la SFR. Las simulaciones EAGLE son herramientas ideales para este propósito, debido a su combinación de volúmenes y resoluciones que permite estudiar la estructura interna de las galaxias al igual que provee de estadística suficiente para explorar diversas propiedades de galaxias a masa estelar fija. En este Capítulo, nos enfocamos en galaxias SF centrales que tienen al menos 10 partículas de gas acretado en los últimos $\approx 100 \mathrm{Myr}$ que vienen de fuentes que no sean fusiones de galaxias, a redshifts $z=0 \mathrm{y}$ $z=1$.

A continuación, resumimos nuestras conclusiones:

- La tasa de acreción de gas correlaciona positivamente con la masa estelar (Figura 4.1) y con la SFR (Figura 4.5), a todo redshift estudiado. También encontramos que, a masa estelar fija, las galaxias con mayor metalicidad de gas están asociadas a menores $\dot{M}_{\text {accr }}$ (Figura 4.1). Estos resultados sumados son consistentes con la FMR (Mannucci et al., 2010).

- Una correlación negativa ajustada es encontrada entre la pendiente interna del RMP (medida en $\left.r / r_{50} \leq 1\right)$ y $\dot{M}_{\text {accr }}$ (Figura 4.2) para todo redshift estudiado. Las galaxias con mayor $\dot{M}_{\text {accr }}$ tienden a tener también perfiles más empinados $(\alpha<0)$. Aún cuando las galaxias cambien su $\dot{M}_{\text {accr }}$ con el tiempo a masa estelar fija, esta anti-correlación no pareciera evolucionar. A radios grandes, $r / r_{50}>1$, encontramos una tendencia de los RMPs a volverse planos a medida que $\dot{M}_{\text {accr }}$ incrementa. Asimismo, se encuentra que esta relación resulta levemente bivaluada para la simulación Ref-L100N1504. Sin embargo, esta tendencia está caracterizada por una dispersión muy grande que nos impide establecer conclusiones firmes al respecto. Se requieren simulaciones de mayor resolución para confirmar esta tendencia.

- Una clara anti-correlación entre la pendiente interna del RMP y $\dot{M}_{\text {accr }}$ permanece incluso cuando se elimina la dependencia con la masa estelar (Figura 4.4).

- La SFR no está tan fuertemente anti-correlacionada con la pendiente interna $\alpha$ como lo está con $\dot{M}_{\text {accr }}$ (Fig 4.6), indicando que esta última es más fundamental en dar forma al RMP.

- También obtenemos una relación entre la fracción de gas neutro y la pendiente interna del RMP (Figura 4.7), pero ésta es menos clara que la hallada para $\dot{M}_{\text {accr }}$. Sin embargo, la fracción de gas es una sustituta útil debido a que es más accesible observacionalmente que la $\dot{M}_{\text {accr }}$.

- Al analizar la RMP en intervalos de redshift y masa estelar, encontramos que galaxias con valores de $\dot{M}_{\text {accr }}$ menores muestran pendientes internas más planas, mientras que galaxias con valores de $\dot{M}_{\text {accr }}$ mayores muestran perfiles más empinados, es decir, con pendientes más negativas (Figura 4.8).

En el futuro, apuntaremos a revelar qué propiedades causan la dispersión de los RMPs a $r / r_{50}>1$. Otro aspecto interesante a investigar como extensión de este trabajo es la distribución bidimensional de abundancias químicas de las galaxias, que aporta información adicional respecto a los resultados obtenidos a partir de la estimación de un promedio radial (Marino et al., 2016; Trayford \& Schaye, 2019). Mostramos este análisis en el Capítulo 5. A pesar de que las suposiciones para el cálculo de $\dot{M}_{\text {accr }}$ fueron simples, es claro que la tasa de acreción de gas juega un papel principal en modificar los RMPs de las galaxias a un grado que esperamos sea observable con una combinación de instrumentos IFS sensibles, estudios de líneas de absorción y observaciones profundas de HI, como por ejemplo en relevamientos a bajo redshift como SAMI (Bryant et al., 2015) y CALIFA (Sánchez et al., 2012), y en el futuro cercano el relevamiento MUSE MAGPI ${ }^{3}$ (por Middle Ages Galaxy Properties with Integral Field Spectroscopy; Foster et al. 2020) a $z \approx 0.3$.

${ }^{3}$ https://magpisurvey.org/ 


\section{Capítulo 5}

\section{Análisis del contenido químico de regiones locales en galaxias simuladas}

En el Capítulo anterior discutimos la importancia que tiene la acreción de gas en el enriquecimiento químico de las galaxias, analizando sus perfiles radiales de metalicidad. Sin embargo, hay varios interrogantes que han quedado abiertos. Por un lado, el hecho de que los perfiles radiales de metalicidad se encuentran más empinados en sus regiones centrales cuanto mayor es la tasa de gas acretado podría estar relacionado con la metalicidad que aporta ese gas entrante, o con su velocidad o momento angular que podría favorecer una formación estelar rápida y posterior enriquecimiento químico (al menos, de manera acelerada con respecto a los procesos que no involucran acreción de gas). Por otro lado, la localización del gas acretado, ya sea en una región en particular o distribuido a lo largo de la galaxia, puede ser consecuencia de distintos caminos en el procesos de enriquecimiento químico.

Para poder responder estos interrogantes es necesario estudiar las galaxias de una manera local o resuelta, de forma que se puedan discriminar regiones cuyos procesos físicos se alteran debido al gas entrante producto de procesos de acreción. Gracias a los avances tecnológicos de los últimos años, tanto en el ámbito observacional como computacional, este tipo de estudios puede realizarse con una estadística suficiente para obtener resultados interesantes (Barrera-Ballesteros et al., 2018). En primera instancia, el estudio de las propiedades resueltas permite inferir el origen de las relaciones globales conocidas (es decir, aquéllas que relacionan las propiedades integradas de las galaxias, como la masa estelar, la SFR y la masa de gas; Tremonti et al. 2004; Schreiber et al. 2015; Boselli et al. 2014), al tiempo que aporta nuevas correlaciones e información al estudio de la formación de las galaxias. En esta línea, e inspiradas en el trabajo observacional de Hwang et al. (2019) (H19, entre otros), profundizamos en el estudio de la metalicidad de las galaxias, realizando diversos análisis de las propiedades locales de galaxias simuladas. Investigamos si la metalicidad a nivel local tiene relaciones similares con otras propiedades de las galaxias al igual que lo tiene a nivel global, como así también si se modifica significativamente debido, principalmente, a la cantidad de masa de gas acretado. Parte de los resultados mostrados en este Capítulo han sido publicados en Collacchioni et al. (2021).

Al igual que en el Capítulo 4, para este estudio utilizamos las simulaciones Ref-L100N1504 y Recal-L25N752 del proyecto EAGLE (ver Sección 2.2), manteniendo los resultados de ambas simulaciones por separado. Esto se debe a las diferencias que tienen ambas simulaciones en los parámetros físicos que se relacionan con el feedback estelar y de AGN (Sección 2.2.4). 


\subsection{Descripción de regiones locales simuladas y sus propiedades}

Desde el punto de vista observacional, para poder afirmar que una zona en particular de una galaxia tiene un valor alto o bajo de cierta propiedad, se analiza si esa zona cuenta con un número mínimo de spaxels con valores similares para dicha propiedad. Para ello, suele tomarse como número mínimo de spaxels unos 15 - 20, lo que equivale a que esa zona tenga dimensiones del orden de $\sim 1$ kpc. Esto surge porque el área angular típica de la función de dispersión de punto ${ }^{1}$ (PSF, por Point Spread Function) tiene $\sim 2.5$ arcsec, que equivale a $\sim 1.5 \mathrm{kpc}$ a $z=0.03$, (Hwang et al., 2019). Actualmente, las simulaciones cosmológicas con las que trabajamos no pueden reproducir los spaxels que se observan, debido a que sus dimensiones se encuentran por debajo de la resolución de las simulaciones (siendo, en nuestro caso, $0.7 \mathrm{kpc}$ para Ref-L100N1504 y $0.35 \mathrm{kpc}$ para RecalL25N752). Sin embargo, sí podemos reproducir con confianza las zonas de $\sim 1 \mathrm{kpc}$. Por este motivo, a partir de aquí haremos un abuso de la notación y utilizaremos el término spaxel para referirnos a las zonas locales simuladas con dimensiones de $1 \mathrm{kpc}$ de lado.

Para generar los spaxels de un grupo de galaxias simuladas, el primer paso es rotar las mismas para que se encuentren de frente (o face-on) respecto del sistema de referencia de la simulación. Esto se hace para imitar la estimación de propiedades locales obtenidas observacionalmente, donde tienen en cuenta las componentes $x y$ espaciales para determinar las dimensiones de las galaxias (H19). Consideramos que el momento angular de la galaxia, $\vec{L}$, será aquél provisto por la componente estelar, por lo que la suma de los momentos angulares de las partículas de estrellas resulta en el momento angular de la galaxia, de la forma

$$
\vec{L}=\sum_{i=1}^{\mathrm{N}} \overrightarrow{r_{i}} \times m_{i} \overrightarrow{v_{i}},
$$

donde la sumatoria recorre todas las partículas de estrellas de las galaxias (siendo $\mathrm{N}$ el número de partículas de estrellas total de cada galaxia), $m_{i}$ es la masa de cada partícula, y $\overrightarrow{r_{i}}$ y $\overrightarrow{v_{i}}$ son, respectivmanete, los vectores posición y velocidad de cada partícula con respecto al centro de la galaxia. Para que la componente $z$ del momento angular se corresponda con el del sistema de referencia, simplemente aplicamos las rotaciones correspondientes tanto a la componente estelar como a la componente gaseosa. Luego de tener las posiciones de todas las partículas (sea gas o estrellas) en un sistema de referencia acorde, limitamos la muestra de partículas hasta $2.5 \times r_{50}$, donde $r_{50}$ es el radio en donde la galaxia alcanza el $50 \%$ de su masa estelar. Esto nos sirve, por un lado, para no considerar regiones muy externas de las galaxias donde el número de partículas es bajo y afectará a la estadística de las propiedades locales. Por otro lado, los telescopios y relevamientos actuales tienden a tomar información hasta $2-2.5 \times r_{50}(\mathrm{H} 19)$.

Por último, realizamos un grillado a las galaxias, centrado en el centro de cada galaxia, para generar nuestros spaxels simulados, los cuales tienen dimensión de $1 \mathrm{kpc} \times 1 \mathrm{kpc}$. Las partículas que queden dentro de cada spaxel serán las que aporten la información de las propiedades locales de nuestras galaxias simuladas. Un esquema del grillado de una galaxia puede verse en la Figura 5.1. No obstante, el tamaño de los spaxels resulta relevante ya que varias de las propiedades que usamos están normalizadas por el área del spaxel (para mayor detalle sobre los valores de las propiedades de acuerdo al tamaño de los spaxels, ver el Apéndice G). Por ejemplo, la densidad superficial estelar, $\Sigma_{\star}$, de un spaxel dado se consigue sumando la masa de todas las partículas de estrellas que pertenecen a ese spaxel y dividiéndolo por la superficie del spaxel (en el caso de que el spaxel sea de $1 \mathrm{kpc} \times 1 \mathrm{kpc}$, la superficie tendrá valor $1 \mathrm{kpc}^{2}$ ). De manera similar, la metalicidad de cada spaxel está determinada por la densidad superficial de masa de oxígeno, $\Sigma_{\mathrm{O}}$, y la densidad superficial de masa de hidrógeno,

\footnotetext{
${ }^{1}$ La función de dispersión de punto (PSF) describe la respuesta de un sistema de imagen a una fuente de punto o punto objeto.
} 
$\Sigma_{\mathrm{H}}$, contenidas en las partículas de gas SF, de la forma

$$
Z=12+\log \left(\frac{\Sigma_{\mathrm{O}}}{16} \frac{1}{\Sigma_{\mathrm{H}}}\right)
$$

Así, trabajamos en este capítulo con la densidad superficial estelar, $\Sigma_{\star}$, la densidad superficial de SFR, $\Sigma_{\text {SFR }}$, la densidad superficial de masa gaseosa, $\Sigma_{\text {gas }}$, la metalicidad de los spaxels, la distancia galactocéntrica proyectada (descrita como $\sqrt{x^{2}+y^{2}}$, con $x$ e $y$ componentes de la posición de los spaxels respecto del centro de la galaxia), y la fracción de gas acretada, $f_{\text {accr }}$ (definida en la Subsección 5.1.1).

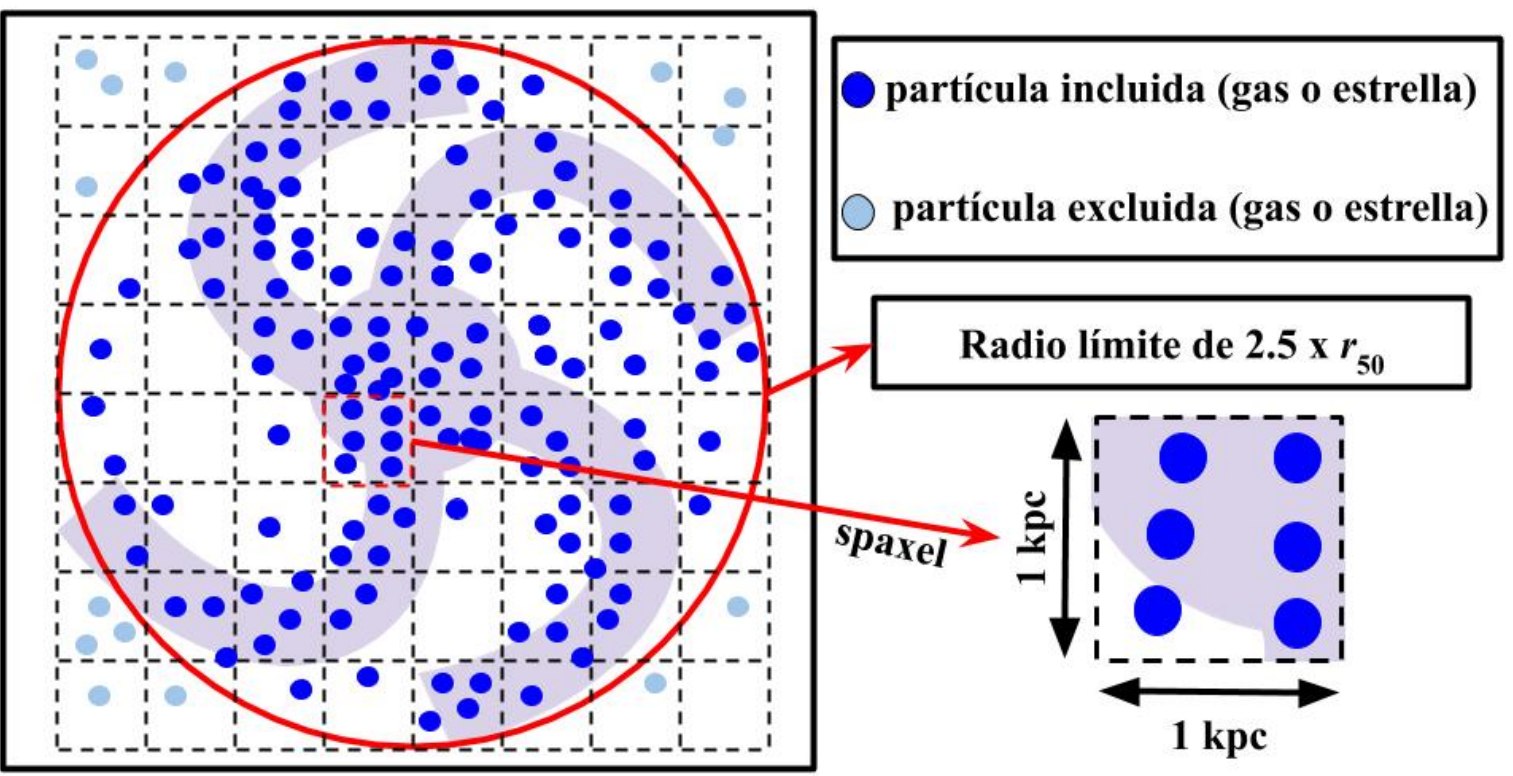

Figura 5.1: Esquema de grillado que se realiza a las galaxias simuladas. El radio límite que se toma para el cálculo de propiedades integradas está representado por el círculo rojo, el cual tiene un radio de $2.5 \times r_{50}$. Los puntos azules representan partículas (ya sea de gas o de estrellas) que son tomadas para calcular las propiedades locales. Mientras que los puntos celestes denotan aquellas partículas (de gas o de estrellas) que quedan por fuera de la selección. En la parte derecha de la Figura puede verse de manera aumentada uno de los spaxels que resulta de realizar el grillado, con dimensión de $1 \mathrm{kpc} \times 1 \mathrm{kpc}$. Las propiedades locales de un spaxel dado se calculan de acuerdo a las partículas que quedan dentro de dicho spaxel.

Dado que el gas de las galaxias satélites resulta afectado por el mayor número de interacciones que sufren las mismas producto del ambiente en que se encuentran, en este Capítulo mostramos los resultados obtenidos de considerar solamente galaxias centrales para sortear esas dificultades. Recordamos que las galaxias centrales son aquéllas que se encuentran en los centros de los pozos de potencial gravitatorio, por lo que se clasifican como galaxias aisladas o que, de pertenecer a un grupo o cúmulo, son las de mayor masa de su ambiente. A su vez, nuestra muestra contiene galaxias que están formando estrellas activamente (en la secuencia principal de galaxias), aquéllas que tengan sSFR $\geq 10^{11} \mathrm{yr}^{-1}$ (Furlong et al., 2015). A estos cortes le sumamos la condición de que los spaxels en sí muestren formación estelar $\left(\Sigma_{\mathrm{SFR}}>0\right)$ y que cuenten con partículas de estrellas $\left(\Sigma_{\star}>0\right)$. Luego de todas estas condiciones, obtenemos una muestra conjunta entre las dos simulaciones de 1719 galaxias a $z=0$, las cuales aportan un total de 262691 spaxels. Mientras que a $z=1$ obtenemos una muestra conjunta de 1876 galaxias, que contribuyen con un total de 288412 spaxels. 


\subsubsection{Fracción de gas acretado}

De manera análoga a como calculamos la masa de gas acretado en el Capítulo 4, necesitamos conocer la masa de gas acretado que tiene cada spaxel simulado. Para ello, debemos seguir no sólo la historia de acreción sino también la posición de las partículas de gas de las galaxias de nuestra muestra. Esto último surge por la necesidad de ubicar a los spaxels espacialmente y, por ende, ubicar a las partículas que pertenecen a esos spaxels. En este sentido, identificamos las partículas de gas de un dado subhalo a una salida determinada y analizamos sus características en un tiempo anterior. Definimos a las partículas de gas acretado como aquéllas que cumplen con:

(a) ser partículas de gas SF en la salida analizada, y

(b) clasificarse como partículas no-SF en la salida anterior, es decir, que a un tiempo previo no se clasifiquen como SF.

Con este criterio, las partículas de gas acretadas resultan material nuevo de la galaxia para formar estrellas, independientemente de la procedencia de estas partículas. Las mismas pueden provenir del mismo progenitor principal que la galaxia analizada, de otra galaxia (por medio de fusiones o interacciones cercanas), o del IGM. De esta manera, la masa de gas acretado definida aquí está compuesta por gas que desencadena formación estelar.

La diferencia que este análisis tiene con respecto al descrito en la Sección 4.2 es que aquí no limitamos la procedencia de las partículas solamente a aquéllas que ya estaban ligadas al progenitor principal, sino que extendemos la definición a partículas ligadas a galaxias satélites que se fusionan con el progenitor o ceden material al mismo, y a las que no están ligadas a ninguna estructura (es decir, provienen del IGM). Así, estamos considerando la acreción suave de gas, que es la principal fuente de acreción de gas en las galaxias (van de Voort et al., 2011; Wright et al., 2020), adicionada con la acreción de material proveniente de satélites para analizar si las fusiones generan un impacto significativo o no al enriquecimimento químico. Dado un spaxel, la $f_{\text {accr }}$ es definida, entonces, como la cantidad de partículas de gas acretado que se encuentran allí dividido la cantidad de partículas de gas SF total en dicho spaxel. Más precisamente, la descripción de la fracción de gas acretado, $f_{\text {accr}}$, resulta

$$
f_{\text {accr }}=\Sigma_{\text {accr }} / \Sigma_{\text {gas }},
$$

donde $\Sigma_{\text {accr }}$ es la densidad superficial de masa de gas acretado en el spaxel (generada mediante las partículas de gas que se identificaron como acretadas) y $\Sigma_{\text {gas }}$ es la densidad superficial de masa de gas total (generada con las partículas de gas SF) en el spaxel. Notar que estrictamente $f_{\text {accr }} \leq 1$.

$\mathrm{Al}$ igual que en la sección 4.2, los subhalos progenitores son identificados usando los árboles de fusión (Jiang et al., 2014; Qu et al., 2017). El intervalo de tiempo entre las salidas de las simulaciones usadas en este Capítulo (Ref-L100N1504 y Recal-L25N752) es de 1.34 Gyr para $z=0 \rightarrow 0.1$ y 0.93 Gyr para $z=1 \rightarrow 1.26$. A pesar de que este cálculo del gas acretado es un poco más abarcativo que el que se desarrolló en el Capítulo 4, este método sigue teniendo la debilidad inherente de no considerar el gas que cae a la galaxia y resulta eyectado antes de que la acreción de gas sea definida en cada salida.

\subsection{Conexión entre relaciones resueltas e integradas}

En esta Sección, construimos relaciones resueltas que tienen relación global análoga conocida, a saber, la MZR y la secuencia principal de galaxias. De esta forma, analizamos la evolución de las relaciones locales y cómo se condicen con sus análogas globales. Asimismo, investigamos si nuestras predicciones son confiables al compararlas con observaciones. 


\subsubsection{La Relación Masa-Metalicidad resuelta}

Como vimos en el Capítulo 1, una de las primeras preguntas que surgen al estudiar propiedades locales de galaxias es si esas propiedades se relacionan entre sí de manera similar como lo hacen sus análogas integradas. La Figura 5.2 muestra la relación entre la metalicidad de los spaxels en función de la $\Sigma_{\star}$ a $z=0$, denotada como rMZR (por resolved Mass-Metallicity Relation). Las simulaciones Ref-L100N1504 y Recal-L25N752 están representadas con colores azul y rojo, respectivamente. Las líneas sólidas muestran la mediana de la relación, mientras que los percentiles 16 - 84 se muestran con sombreado. A su vez, mostramos los resultados observacionales de Sánchez et al. (2013) con círculos grises, de Barrera-Ballesteros et al. (2016) con estrellas negras, y de H19 para galaxias en los rangos de masa estelar de $10^{9} \leq M_{\star} / \mathrm{M}_{\odot} \leq 10^{9.5}$ con línea amarilla a trazos, $10^{9.5} \leq M_{\star} / \mathrm{M}_{\odot} \leq 10^{10}$ con línea rosa a trazos y $M_{\star} / \mathrm{M}_{\odot} \geq 10^{10}$ con línea violeta a trazos. Debido a que la escala de la rMZR puede variar dependiendo de los indicadores de metalicidad que se utilicen sin que esto altere significativamente la forma de la relación (Rosales-Ortega et al., 2012), se realizó un desplazamiento de las metalicidades observadas de un valor de +0.6 dex para que la normalización de la rMZR se asemeje con las observacionales (similar a como se hizo en Trayford \& Schaye 2019). Cabe aclarar que nuestros cálculos de metalicidad están pesados por masa y no por intensidad de la luz, por lo que nuestro trabajo se concentra principalmente en los cambios que sufre la metalicidad y no tanto en los valores que toma.

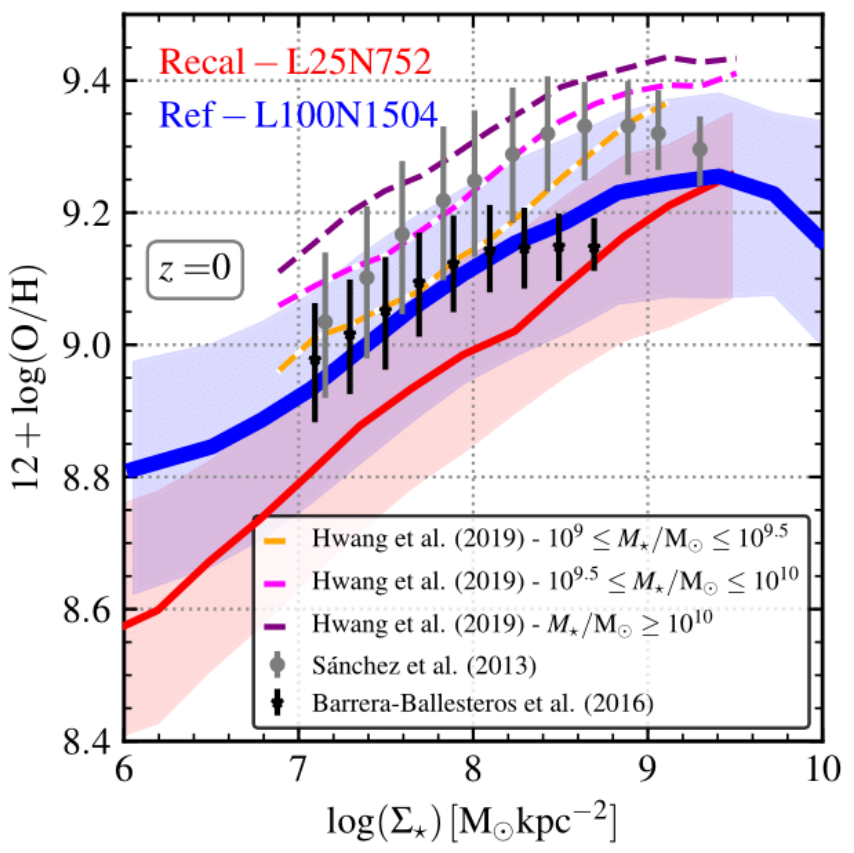

Figura 5.2: Relación Masa-Metalicidad resuelta (rMZR) a $z=0$. Las simulaciones Ref-L100N1504 y RecalL25N752 están representadas con colores azul y rojo, respectivamente. Las líneas sólidas denotan los valores medianos de la relación, mientras que las sombras muestran los percentiles 16 - 84. Los círculos grises represetan los valores medianos de las observaciones de Sánchez et al. (2013), realizadas con el relevamiento CALIFA, y las estrellas negras denotan los valores medianos de las observaciones de Barrera-Ballesteros et al. (2016), realizadas con el relevamiento MaNGA. Las líneas a trazos amarilla, rosa y violeta muestran los valores medianos de la relación para galaxias con masa estelar de $10^{9} \leq M_{\star} / \mathrm{M}_{\odot} \leq 10^{9.5}, 10^{9.5} \leq M_{\star} / \mathrm{M}_{\odot} \leq 10^{10} \mathrm{y}$ $M_{\star} / \mathrm{M}_{\odot} \geq 10^{10}$, respectivamente, obtenidos por H19 utilizando el relevamiento MaNGA. Se ha aplicado un corrimiento de +0.6 dex a las observaciones aquí mostradas.

Entonces, si nos concentramos en las pendientes de las relaciones presentadas en la Figura 5.2, 
podemos ver que galaxias de masa baja e intermedia $\left(M_{\star} \lesssim 10^{10.5} \mathrm{M}_{\odot}\right)$ muestran crecimientos lineales (en la escala logarítmica), es decir, que al aumentar su $\Sigma_{\star}$ también crece la metalicidad de sus spaxels. Para galaxias de alta masa $\left(M_{\star} \gtrsim 10^{10.5} \mathrm{M}_{\odot}\right)$ se observa un aplanamiento de la relación, tanto en las observaciones como en la simulación Ref-L100N1504, que es aquélla con galaxias de mayor masa estelar. Esto nos está indicando que las tasas de enriquecimiento químico que tienen los spaxels de distintos valores de $\Sigma_{\star}$ son similares a lo que se infiere observacionalmente. Por consiguiente, podemos analizar si estas tasas de enriquecimiento se ven modificadas en el tiempo, o si resulta un valor intrínseco a la cantidad de $\Sigma_{\star}$ que tiene un spaxel.
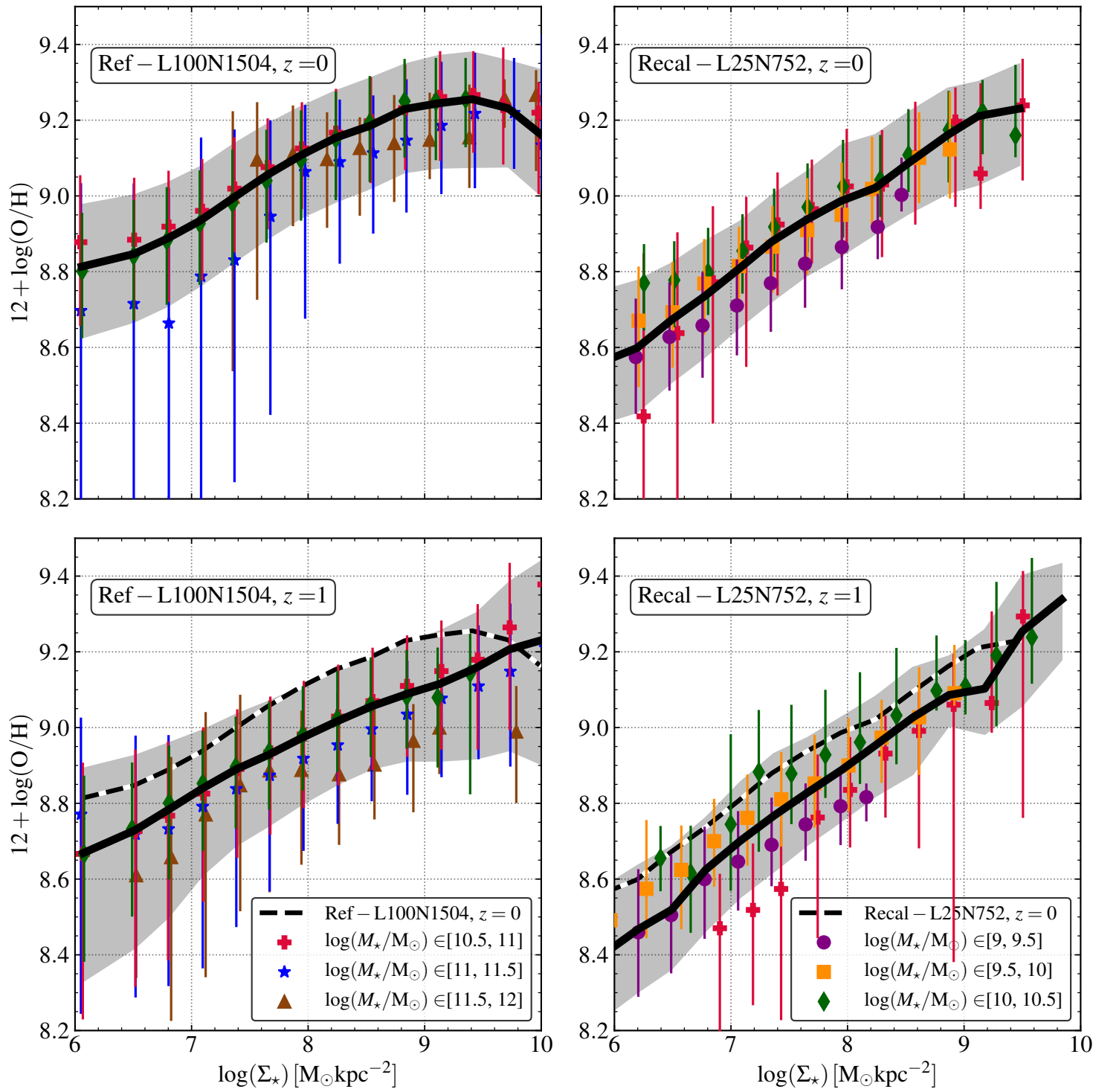

Figura 5.3: Metalicidad de los spaxels en función de la densidad superficial de masa estelar, $\Sigma_{\star}$, a redshift $z=0$ (paneles superiores) y $z=1$ (paneles inferiores). Los paneles izquierdos muestran los resultados de la simulación Ref-L100N1504, mientras que los paneles derechos muestran los resultados de la simulación RecalL25N752. La línea negra muestra los valores medios de la relación, mientras que la sombra gris sus percentiles 16 - 84. Los círculos violeta, cuadrado naranja, rombo verdes y signos “+” rosas denotan los valores medios de la relación para galaxias en determinado rango de masa estelar (como se muestra en la figura). Las barras de error corresponden a los percentiles 16 - 84 de cada rango de masa estelar correspondiente. Las líneas negras a trazos en los paneles inferiores muestran la mediana de la relación a $z=0$ (para cada simulación). 
La Figura 5.3 muestra los valores medianos de la rMZR para las simulaciones Ref-L100N1504 (paneles izquierdos) y Recal-L25N752 (paneles derechos). Los paneles superiores son los resultados a $z=0$, mientras que los inferiores muestran aquéllos a $z=1$. Los círculos violeta, cuadrados amarillos, rombos verdes, símbolos "+" magenta, estrellas azules y triángulos marrones representan valores de la mediana de la relación para galaxias en intervalos de rangos de masa estelar de 0.5 dex, como se ve en la leyenda. En los paneles inferiores puede verse la mediana de la relación a $z=0$ en línea negra a trazos para las simulaciones Ref-L100N1504 (izquierda) y Recal-L25N752 (derecha). Si observamos los valores medianos de toda la muestra (líneas negras sólidas), podemos notar que la forma de la relación no se ve significativamente alterada al transcurrir el tiempo. Sin embargo, tanto Ref-L100N1504 como Recal-L25N752 muestran una disminución en la normalización de $\sim 0.2$ dex al comparar los resultados a $z=0$ y a $z=1$ (como puede verse con ayuda de las líneas negras a trazos en los paneles inferiores). Dicho de otra manera, a $\Sigma_{\star}$ fija, los spaxels aumentan su metalicidad al disminuir el redshift. Esta evolución de la rMZR es similar a la observada para la MZR global, donde el cambio de normalización de $z=1 \rightarrow 0$ es, también, de 0.2 dex (esto puede verse tanto en los resultados generados por SAG, mostrados en el Capítulo 3, como también para la MZR obtenida por las simulaciones EAGLE estudiada en Guo et al. 2016). En este sentido, es posible que la MZR global sea una consecuencia de la rMZR dado que el grado de evolución en magnitud resultan similares.

Si nos detenemos en los valores medianos de la rMZR para galaxias en rangos de masa estelar específicos, podemos observar dos cosas. Por un lado, galaxias con distintos rangos de masa estelar muestran la misma tendencia que la rMZR construida con todas las galaxias, es decir, los spaxels aumentan su metalicidad a medida que aumentan su $\Sigma_{\star}$. Por otro lado, no pareciera haber una diferencia notoria en las pendientes de la rMZR para cada rango de masa estelar, excepto para uno de los rangos masivos de la simulación Ref-L100N1504 a $z=0$ (denotado con estrellas azules) y para la simulación Recal-L25N752. Sin embargo, estos valores caen dentro de la dispersión de la relación. Además, puede verse que hay una tendencia a que spaxels provenientes de galaxias con menor masa estelar muestren una metalicidad menor a aquéllos que provienen de galaxias de mayor masa (a $\Sigma_{\star}$ fija), análogo a lo que encuentra H19.

\subsubsection{La Secuencia Principal resuelta en galaxias}

Otra relación global importante de las galaxias es la llamada Secuencia Principal, donde se encuentra que galaxias con mayor masa estelar presentan a su vez mayor SFR. Esta relación tiene su contraparte resuelta, la cual podemos ver en la Figura 5.4. Las líneas sólidas representan la mediana de la Secuencia Principal resuelta (rMS, por resolved Main Sequence) a $z=0$, mientras que las sombras muestran los percentiles 16 - 84. Las simulaciones Ref-L100N1504 y Recal-L25N752 se denotan con los colores azul y rojo, respectivamente. A las predicciones de las simulaciones se superponen las observaciones de Cano-Díaz et al. (2016) (línea negra a trazos) y Hsieh et al. (2017) (línea gris punteada a trazos). En la Figura puede verse que, para valores de $\Sigma_{\star}$ bajos $\left(\lesssim 10^{7.5} \mathrm{M}_{\odot} \mathrm{kpc}^{-1}\right)$ y altos $\left(\gtrsim 10^{9} \mathrm{M}_{\odot} \mathrm{kpc}^{-1}\right)$, la simulación Ref-L100N1504 predice valores de $\Sigma_{\mathrm{SFR}}$ mayores que RecalL25N752 ( 0.5 dex $)$. Sin embargo, ambas simulaciones están en acuerdo entre sí y con las observaciones en el rango intermedio de $\Sigma_{\star}$. En el rango en que se aprecian las mayores diferencias no existen límites observacionales y, por lo tanto, es difícil a esta altura decir si una simulación sigue más cercanamente las observaciones en esos extremos. Nuevamente, encontramos que las tendencias de la rMS se asemejan a la secuencia principal global, lo que podría ser indicio de que la rMS determina la secuencia principal global. Por lo tanto, analizamos si esta relación evoluciona o no con el tiempo.

La Figura 5.5 muestra la rMS a $z=0$ (paneles superiores) y $z=1$ (paneles inferiores), discriminando por galaxias en distintos rangos de masa estelar. Las líneas negras sólidas denotan la mediana de la relación, mientras el sombreado representa los percentiles $16-84$. Los paneles de la izquierda muestran la mediana de la relación para la simulación Ref-L100N1504 y los paneles derechos, para 
Recal-L25N752. Las medianas de la relación para galaxias en determinado rango de masa estelar, desde $M_{\star} \approx 10^{9} \mathrm{M}_{\odot}$ hasta $M_{\star} \approx 10^{12} \mathrm{M}_{\odot}$ en rangos de 0.5 dex en escala logarítmica, están representadas por los círculos violetas, cuadrados amarillos, rombos verdes, signos "+" rosas, estrellas azules y triángulos marrones, respectivamente. En los paneles inferiores puede verse la mediana de la relación a $z=0$ en línea negra a trazos para las simulaciones Ref-L100N1504 (izquierda) y Recal-L25N752 (derecha). A pesar de las diferencias que mencionamos anteriormente entre las rMS de ambas simulaciones, las mismas comparten la tendencia de predecir spaxels con mayor $\Sigma_{\mathrm{SFR}}$ a medida que aumentan su $\Sigma_{\star}$. El aplanamiento de la relación en la región de valores bajos de $\Sigma_{\star}$ puede deberse al hecho de que las galaxias que componen esa relación, $M_{\star} \geq 10^{10} \mathrm{M}_{\odot}$, cuentan con un feedback de AGN que empieza a tomar relevancia en comparación con las galaxias de Recal-L25N752 (Trayford \& Schaye, 2019). A su vez, ambas simulaciones presentan una evolución de la rMS con el redshift (diferencia de $\sim 2.5$ dex entre las líneas negras sólidas y a trazos), de manera que los spaxels disminuyen su $\Sigma_{\text {SFR }}$ al tiempo que el redshift decrece, a $\Sigma_{\star}$ fija. Este comportamiento es análogo al mostrado por la secuencia principal global, en donde las galaxias presentan, a masa estelar fija, una disminución de la SFR a medida que pasa el tiempo (Leslie et al., 2020).

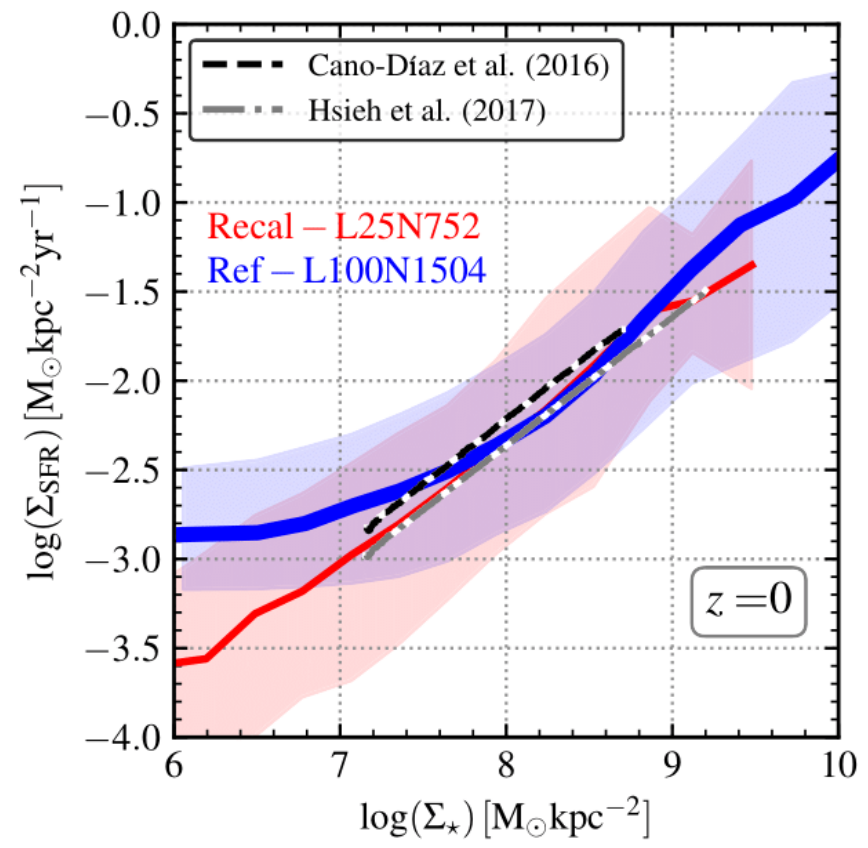

Figura 5.4: Secuencia principal resuelta a $z=0$. Las simulaciones Ref-L100N1504 y Recal-L25N752 están representadas con colores azul y rojo, respectivamente. Las líneas sólidas denotan los valores medianos de la relación, mientras que las sombras muestran los percentiles 16-84. Las observaciones de Cano-Díaz et al. (2016), realizadas con el relevamiento CALIFA, están representadas con la línea negra a trazos, mientras que las observaciones de Hsieh et al. (2017), obtenidas a partir del relevamiento MaNGA, se muestran con línea gris punteada a trazos.

Analizando la rMS para galaxias en determinados rangos de masa estelar, notamos que, en general, las rMS de galaxias con $M_{\star}$ alta $\left(\gtrsim 10^{10.5} \mathrm{M}_{\odot}\right)$ se encuentran por debajo de la rMS total. Mientras que galaxias con $M_{\star}$ baja $\left(\lesssim 10^{9.5} \mathrm{M}_{\odot}\right)$ se encuentran por encima de la rMS total. Esto puede estar relacionado con la SSFR de las galaxias, la cual es mayor a menor masa estelar. En este sentido, los spaxels con menor $\Sigma_{\star}$ están asociados a una producción de estrellas más eficiente que los spaxels con mayor $\Sigma_{\star}$. Sin embargo, estos valores se encuentran dentro de la dispersión de la rMS total, en donde estas diferencias son menores. 

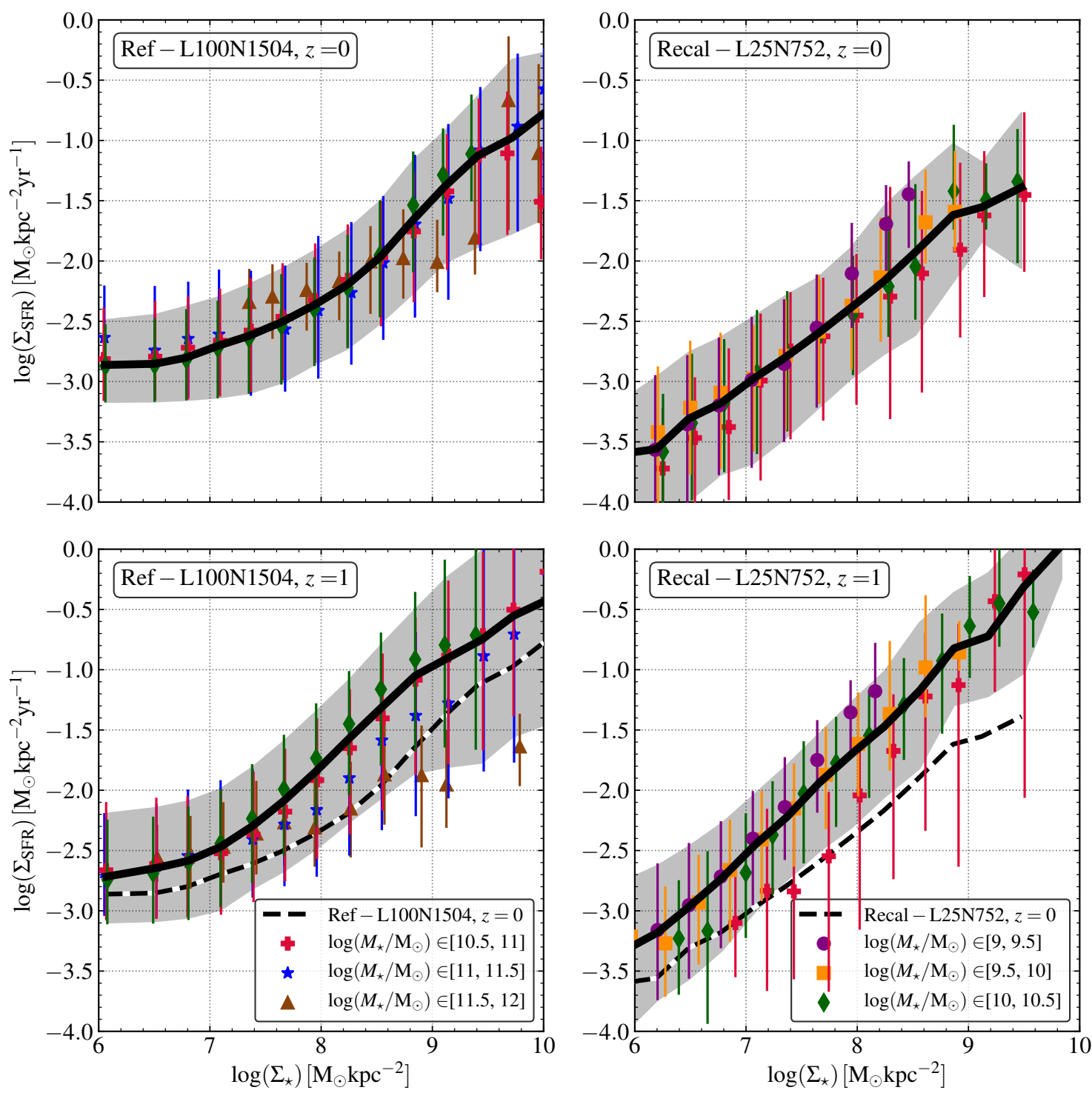

Figura 5.5: Secuencia Principal de los spaxels de las galaxias simuladas a redshift $z=0$ (paneles superiores) y $z=1$ (paneles inferiores). Los paneles izquierdos muestran los resultados de la simulación Ref-L100N1504, mientras que los paneles derechos muestran los resultados de la simulación Recal-L25N752. La línea negra muestra los valores medios de la relación, mientras que la sombra gris sus percentiles 16 - 84. Los círculos violeta, cuadrado naranja, rombo verdes, signos “+” rosas, estrellas azules y triángulos marrones denotan los valores medios de la relación para galaxias en determinado rango de masa estelar (como se muestra en la figura). Las barras de error corresponden a los percentiles 16 - 84 de cada rango de masa estelar correspondiente. Las líneas negras punteadas en los paneles inferiores muestran la mediana de la relación a $z=0$ (para cada simulación)

En esta sección estudiamos la rMZR y la rMS a $z \leq 1$. Por un lado, encontramos que la pendiente de la rMZR simulada está en acuerdo con las observaciones a $z=0$, indicando que las tasas de evolución química son similares a las inferidas observacionalmente. Además, la rMS simulada muestra un muy buen acuerdo con las observaciones, tanto en pendiente como en normalización. Por otro lado, encontramos que estas relaciones locales reproducen comportamientos similares a sus relaciones análogas globales (la MZR y la secuencia principal, respectivamente) hasta, al menos, $z \lesssim 1$. De esta manera, podemos concluir que las relaciones globales pueden ser inferidas de las locales, al 
tiempo que se cuenta con mayor detalle de los procesos físicos que ocurren en las galaxias. A continuación, presentamos los resultados del estudio del efecto que tiene el gas acretado a escala local y las consecuencias que trae aparejadas.

\subsection{Fracción de gas acretado como reguladora de la metalicidad}

Uno de los objetivos de este Capítulo es mostrar si la metalicidad puede ser una buena estimadora de la acreción de gas. Para poder responder esta cuestión, algo que tenemos que preguntarnos primero es si el gas entrante está aportando o no nuevos elementos químicos al gas que ya se encuentra en la galaxia. Para ello, presentamos la Figura 5.6, que muestra la relación entre la metalicidad de los spaxels y su fracción de gas acretada $\left(Z-f_{\text {accr }}\right)$. Los valores medianos de la relación son representados con líneas sólidas negras, mientras que las sombras denotan los percentiles 16 - 84. Las simulaciones Ref-L100N1504 y Recal-L25N752 se muestran en los paneles izquierdos y derechos, respectivamente. Las relaciones se muestran tanto a $z=0$ (paneles superiores) como a $z=1$ (paneles inferiores). En los paneles inferiores puede verse la mediana de la relación a $z=0$ en línea negra a trazos para las simulaciones Ref-L100N1504 (izquierda) y Recal-L25N752 (derecha). Al igual que en las Figuras 5.3 y 5.5, mostramos los valores medianos de la relación distinguiendo spaxels de galaxias en rangos de masa estelar de a 0.5 dex en escala logarítmica, tomando galaxias desde $M_{\star} \approx 10^{9} \mathrm{M}_{\odot}$ hasta $M_{\star} \approx 10^{12} \mathrm{M}_{\odot}$. En los paneles pequeños, puede verse la función de probabilidad de densidad (PDF, por probability density function) de la $f_{\text {accr }}$ para cada caso.

En esta Figura podemos notar tres cosas importantes. La primera es la anti-correlación entre los valores medianos de las cantidades involucradas en la relación (líneas negras) para todos los casos analizados, tal que spaxels con $f_{\text {accr }}$ mayores muestran valores de metalicidad menores. Esta anticorrelación indica que las regiones de las galaxias donde se concentra la mayor cantidad de gas acretado presenta una metalicidad menor, en comparación con regiones donde la acreción de gas es poca o incluso nula. Para determinar cuán anti-correlacionadas se encuentran estas relaciones, calculamos el rango del coeficiente de correlación de Spearman $\left(\mathrm{R}_{\mathrm{s}}\right)$, el cual indica si una relación es correlacionada positiva $\left(\mathrm{R}_{\mathrm{s}} \sim 1\right)$, anti-correlacionada $\left(\mathrm{R}_{\mathrm{s}} \sim-1\right)$, o nada correlacionada $\left(\mathrm{R}_{\mathrm{s}} \sim 0\right)$. En nuestro caso, hallamos que la simulación Ref-L100N1504 tiene un $\mathrm{R}_{\mathrm{s}} \approx-0.45 \mathrm{a} z=0$ y $\mathrm{R}_{\mathrm{s}} \approx-0.54$ a $z=1$, mientras que Recal-L25N752 tiene $\mathrm{R}_{\mathrm{s}} \approx-0.53 \mathrm{a} z=0$ y $\mathrm{R}_{\mathrm{s}} \approx-0.59$ a $z=1$. Con esto podemos afirmar que la metalicidad de los spaxels anti-correlaciona con la $f_{\text {accr }}$ de los mismos, al menos hasta $z \leq 1$. Hay una leve tendencia de invertir este comportamiento para spaxels con valores de $f_{\text {accr }} \leq 0.15$, sobretodo para Recal-L25N752, aunque podría deberse a la menor cantidad de spaxels en ese rango (de acuerdo a los PDFs).

En segundo lugar observamos los valores medianos de la relación $Z-f_{\text {accr }}$ para galaxias en distintos rangos de masa estelar. Analizando en detalle estos valores, podemos ver que para galaxias con $M_{\star} \geq 10^{9.5} \mathrm{M}_{\odot}$ la anti-correlación se mantiene. Para el caso particular de las galaxias con $M_{\star} \leq 10^{9.5} \mathrm{M}_{\odot}$, la anti-correlación se ve débil, aunque tiene un valor de $\mathrm{R}_{\mathrm{s}} \approx-0.36$. A su vez, se observa que, en general, los spaxels tienen mayor metalicidad al provenir de galaxias con mayor masa estelar (a $f_{\text {accr }}$ fija).

Por último, en los paneles inferiores podemos analizar la evolución de la relación con el tiempo. En ambas simulaciones no se aprecia una gran diferencia en pendiente de esta relación. Por otro lado, hay una débil evolución de la normalización de esta relación, de manera que los spaxels tienen mayor metalicidad al disminuir el redshift, a $f_{\text {accr }}$ fija. Esta evolución es de $\approx 0.1$ dex en ambas simulaciones.

De esta manera, podemos ver que, por un lado, la metalicidad de los spaxels decrece con la $f_{\text {accr. }}$. Esto indicaría que, en general, la $f_{\text {accr }}$ no aporta nuevos metales al ISM de las galaxias, sino que más bien diluye los metales presentes cuando se encuentra en grandes cantidades (en comparación con el gas previo). Por otro lado, hay una tendencia de que los spaxels aumenten su metalicidad al pasar el tiempo (a $f_{\text {accr }}$ fija), lo cual podría estar relacionado al tiempo en que el medio se enriquece 
químicamente. Sin embargo, estas conclusiones podrían deberse a la intervención de alguna otra propiedad. A continuación, mostramos los cambios que la metalicidad muestra en función de la $f_{\text {accr }}$ y de la $\Sigma_{\text {SFR }}$.
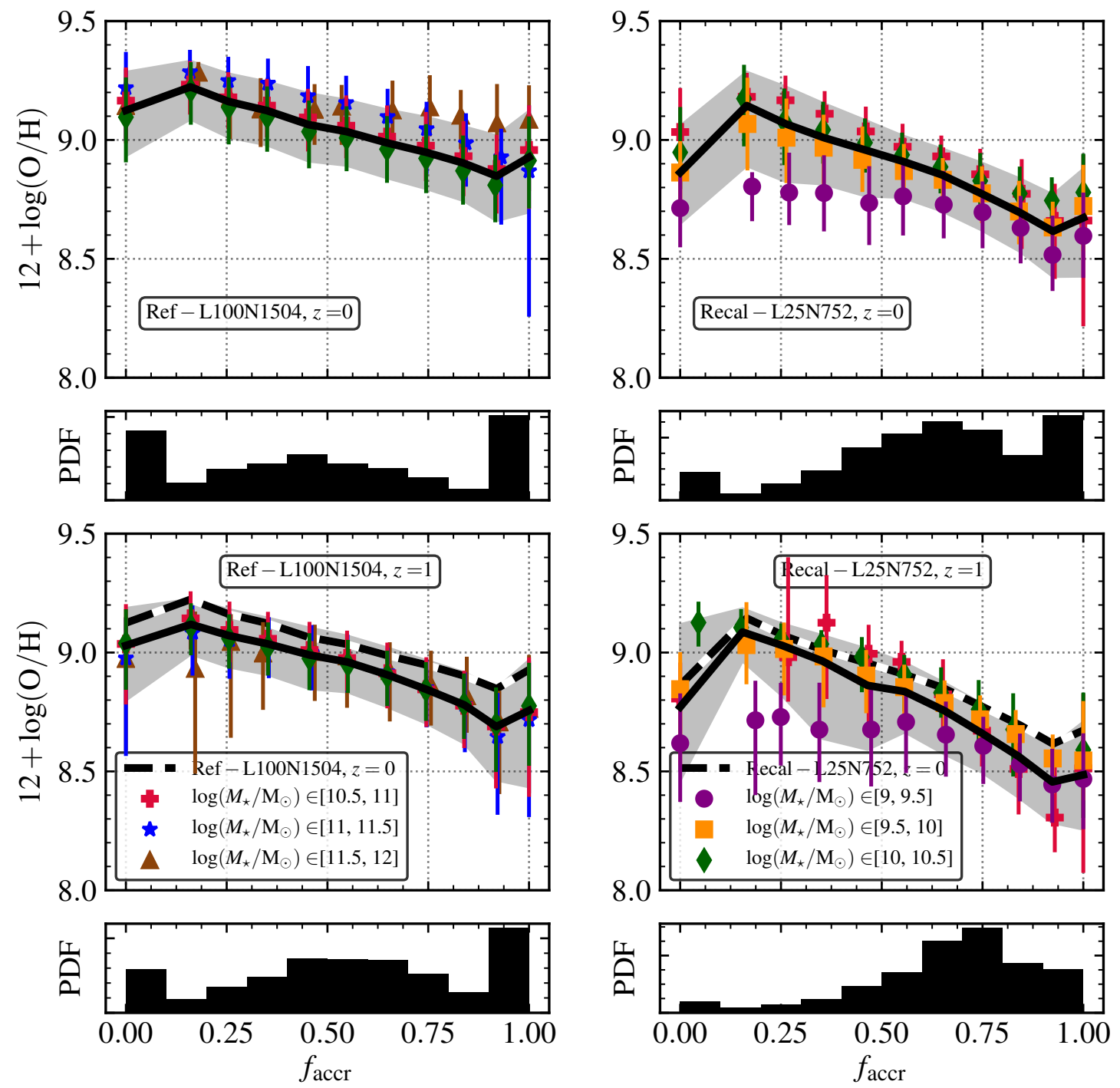

Figura 5.6: Metalicidad de los spaxels en función de la fracción de gas acretada, $f_{\text {accr }}$, a redshift $z=0$ (paneles superiores) y $z=1$ (paneles inferiores). Los paneles izquierdos muestran los resultados de la simulación RefL100N1504, mientras que los paneles derechos muestran los resultados de la simulación Recal-L25N752. La línea negra muestra los valores medios de la relación, mientras que la sombra gris sus percentiles 16 - 84 . Los círculos violeta, cuadrado naranja, rombo verdes y signos "+" rosas denotan los valores medios de la relación para galaxias en determinado rango de masa estelar (como se muestra en la figura). Las barras de error corresponden a los percentiles 16 - 84 de cada rango de masa estelar correspondiente. Las líneas negras punteadas en los paneles inferiores muestran la mediana de la relación a $z=0$ (para cada simulación). Se muestra la PDF de la $f_{\text {accr }}$ para todos los casos en los paneles inferiores a los principales. 


\subsubsection{Análisis de los residuos de la metalicidad}

Debido a que nos interesa estudiar los cambios que la $f_{\text {accr }}$ puede generar sobre la metalicidad del gas, nos concentramos en la contribución de todos los spaxels sin distinguir por masa estelar de la galaxia que los conforma. Además, para realizar este análisis vamos a utilizar una definición de residuos similar a la Ecuación 4.3. En este caso, los residuos de metalicidad, $\Delta(\mathrm{O} / \mathrm{H})$, se definen como

$$
\Delta(\mathrm{O} / \mathrm{H})=\mathrm{O} / \mathrm{H}-\operatorname{med}(\mathrm{O} / \mathrm{H})_{\mathrm{rMZR}}
$$

donde $\mathrm{O} / \mathrm{H}$ es la metalicidad propia de cada spaxel y $\operatorname{med}(\mathrm{O} / \mathrm{H})_{\mathrm{rMZR}}$ es la metalicidad que se espera que un spaxel tenga a un dado $\Sigma_{\star}$ (a partir de la rMZR). Una vez definidos nuestros residuos de metalicidad, procedemos a graficarlos en función de la $f_{\text {accr }}$ en la Figura 5.7. La relación se muestra a $z=0$ (panel izquierdo) y a $z=1$ (panel derecho) para las simulaciones Ref-L100N1504 y RecalL25N752 en azul y rojo, respectivamente. Los valores medios de la relación se denotan con líneas sólidas, mientras que las sombras muestran la dispersión en percentiles de $16-84$. Recordemos que las galaxias de Recal-L25N752 tienen masas estelares por encima de $10^{9} \mathrm{M}_{\odot}$, mientras que las de Ref-L100N1504 tienen masas estelares mayores a $10^{10} \mathrm{M}_{\odot}$.

En esta Figura podemos ver que, independientemente del redshift y la simulación, $\Delta(\mathrm{O} / \mathrm{H})$ disminuye con la $f_{\text {accr. }}$. Esta anti-correlación nos está indicando que, para valores altos de $f_{\text {accr }}(>0.5)$, la metalicidad de los spaxels es menor a la esperada, y esta diferencia se agranda con el aumento de la $f_{\text {accr }}$. A su vez, spaxels con valores bajos de $f_{\text {accr }}(<0.5)$ presentan valores de metalicidad por encima de lo esperado, aumentando esta diferencia a medida que $f_{\text {accr }}$ decrece. Para cuantificar esto, volvemos a estimar el $R_{\mathrm{s}}$, encontrando que vale -0.46 a $z=0$ para Ref-L100N1504 y -0.42 para Recal-L25N752, mientras que a $z=1$ vale -0.53 y -0.45 , respectivamente. En cuanto a la evolución en el tiempo de la relación, se observa que para $f_{\text {accr }} \lesssim 0.5$ los valores de $\Delta(\mathrm{O} / \mathrm{H})$ aumentan levemente $(\approx 0.05$ dex $)$, mientras que para $f_{\text {accr }} \approx 0.5$ los valores de $\Delta(\mathrm{O} / \mathrm{H})$ decrecen levemente $(\approx 0.05$ dex $)$. Esto significa que a mayor $z$ la metalicidad es levemente más sensible a la $f_{\text {accr }}$ en comparación a tiempos más recientes.
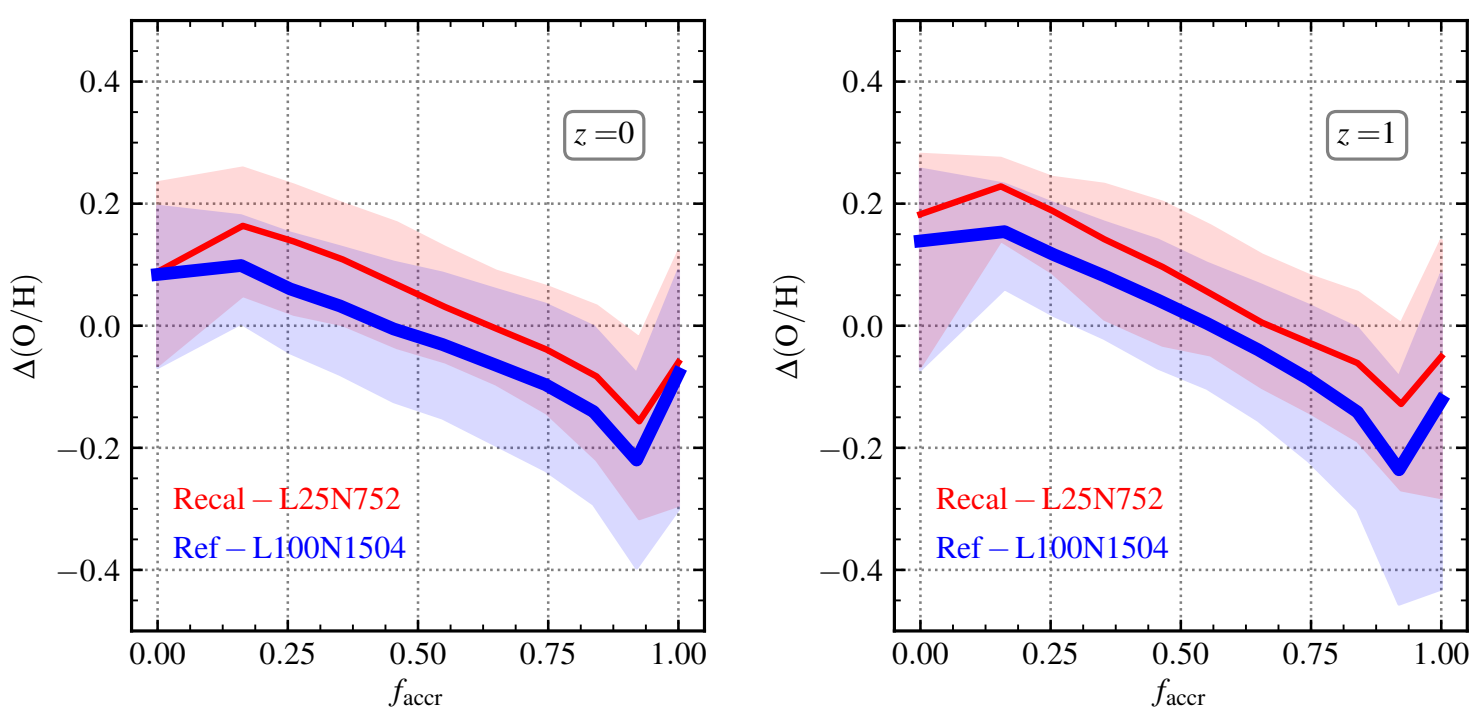

Figura 5.7: Residuos de metalicidad, $\Delta(\mathrm{O} / \mathrm{H})$, en función de la fracción de gas acretado de los spaxels, $f_{\text {accr }}$ a redshift $z=0$ (panel izquierdo) y $z=1$ (panel derecho). Los resultados de la simulación Ref-L100N1504 se encuentran en color azul, mientras que los de Recal-L25N752 se muestran en color rojo. Las líneas sólidas muestran los valores medios de la relación, mientras que las sombras grises sus percentiles $16-84$. 
La anti-correlación que obtuvimos entre los residuos de la metalicidad y la $f_{\text {accr }}$ podría deberse a alguna otra relación entre la metalicidad y otra propiedad galáctica local. Una propiedad que ha resultado estar sumamente relacionada con la masa de gas y la metalicidad es la SFR (ver Capítulo 1 y Capítulo 4). Entonces, procedemos a analizar si los residuos de metalicidad sufren algún cambio cuando se los grafica en función de la $\Sigma_{\mathrm{SFR}}$ (relación $\Delta(\mathrm{O} / \mathrm{H})-\Sigma_{\mathrm{SFR}}$ ). En la Figura 5.8, vemos las predicciones de las simulaciones Ref-L100N1504 (color azul) y Recal-L25N752 (color rojo) para la relación $\Delta(\mathrm{O} / \mathrm{H})-\Sigma_{\mathrm{SFR}}$ a $z=0 \mathrm{y} z=1$ en los paneles izquierdo y derecho, respectivamente. Las líneas sólidas denotan los valores medianos de la relación, mientras que las sombras representan la dispersión de la misma, usando los percentiles 16 - 84 .
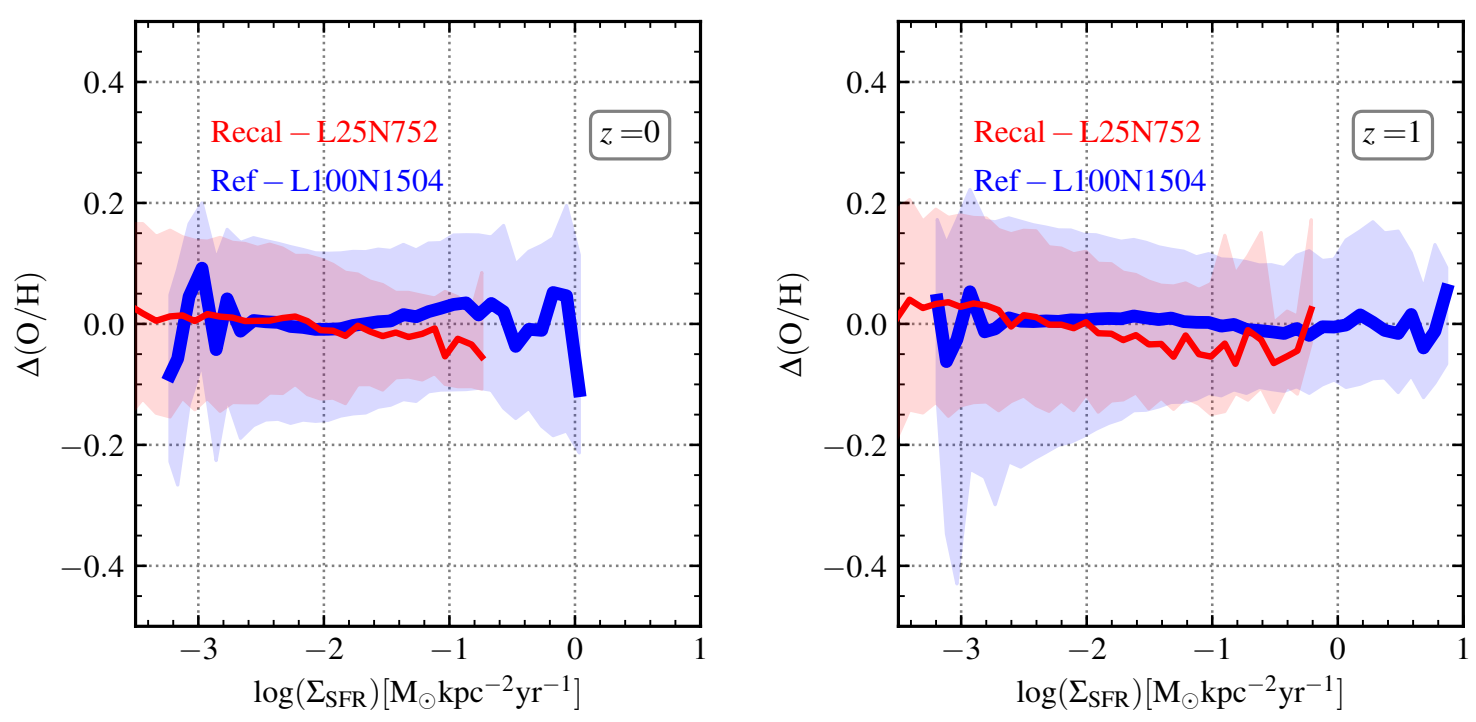

Figura 5.8: Residuos de metalicidad, $\Delta(\mathrm{O} / \mathrm{H})$, en función de la densidad superficial de SFR de los spaxels, $\Sigma_{\mathrm{SFR}}$, a redshift $z=0$ (panel izquierdo) y $z=1$ (panel derecho). Los resultados de la simulación Ref-L100N1504 se encuentran en color azul, mientras que los de Recal-L25N752 se muestran en color rojo. Las líneas sólidas muestran los valores medios de la relación, mientras que las sombras grises sus percentiles $16-84$.

No pareciera haber una correlación entre los residuos de la metalicidad y la $\Sigma_{\mathrm{SFR}}$ para ninguna simulación. Hay una leve tendencia para Recal-L25N752 a que la $\Delta(\mathrm{O} / \mathrm{H})$ decrezca con la $\Sigma_{\mathrm{SFR}}$, pero no resulta significativa comparada con la la relación $\Delta(\mathrm{O} / \mathrm{H})-f_{\text {accr }}$. Más aún, al analizar los coeficientes de Spearman vemos que a $z=0$ valen $\mathrm{R}_{\mathrm{s}} \approx 0.04$ para Ref-L100N1504 y $\mathrm{R}_{\mathrm{s}} \approx-0.04$ para RecalL25N752, mientras que a $z=1$ estos coeficientes valen $\mathrm{R}_{\mathrm{s}} \approx 0.04$ y $\mathrm{R}_{\mathrm{s}} \approx-0.14$, respectivamente. Estos valores son más cercanos a 0 de lo que obtenemos para la relación $\Delta(\mathrm{O} / \mathrm{H})-f_{\text {accr }}$, por lo que podemos concluir que $\Delta(\mathrm{O} / \mathrm{H})$ no se encuentra correlacionada con la $\Sigma_{\mathrm{SFR}}$. De esta manera, encontramos que la metalicidad de los spaxels resulta más sensible a los cambios en la componente gaseosa producida por la acreción de gas, que por la $\Sigma_{\mathrm{SFR}}$. Estos resultados se asemejan a los encontrados al analizar los perfiles de metalicidad radial, descritos en el Capítulo 4, en donde la relación global entre la metalicidad y la tasa de acreción de gas es más fuerte que la relación entre la metalicidad y la SFR.

\subsection{Regiones de acreción de gas en galaxias}

En la sección anterior encontramos que la metalicidad del gas acretado suele ser menor a la metalicidad del medio, diluyendo así la composición química del gas de las galaxias. En este sentido, la metalicidad resulta sumamente sensible a la acreción de gas, en comparación con otras propiedades 
como la $\Sigma_{\mathrm{SFR}}$. A pesar de tener una idea de cómo se distribuyen los metales en las galaxias, como vimos en el Capítulo 4, la distribución del gas acretado podría ser diversa. Es por eso que en esta Sección nos enfocamos en analizar la distribución de la $f_{\text {accr }}$ en las galaxias, con el fin de identificar si localmente se encuentran resultados similares al estudio de los RMPs del Capítulo 4. La Figura 5.9 muestra el porcentaje de spaxels de una determinada clase en función de la distancia proyectada galactocéntrica (recordemos que esta propiedad está construida a partir de las componentes de posición $x$ e $y$, respecto del centro de la galaxia, de la forma $\sqrt{x^{2}+y^{2}}$ ). Los paneles superiores muestran la relación a $z=0$, mientras que los inferiores lo hacen para $z=1$. Las líneas negra sólida, verde punteada a trazos, naranja a trazos y violeta punteada muestran la relación para spaxels que tengan fracción de gas en el rango de $f_{\text {accr }} \leq 0.25,0.25 \leq f_{\text {accr }} \leq 0.5,0.5 \leq f_{\text {accr }} \leq 0.75$ y $f_{\text {accr }} \geq 0.75$, respectivamente.
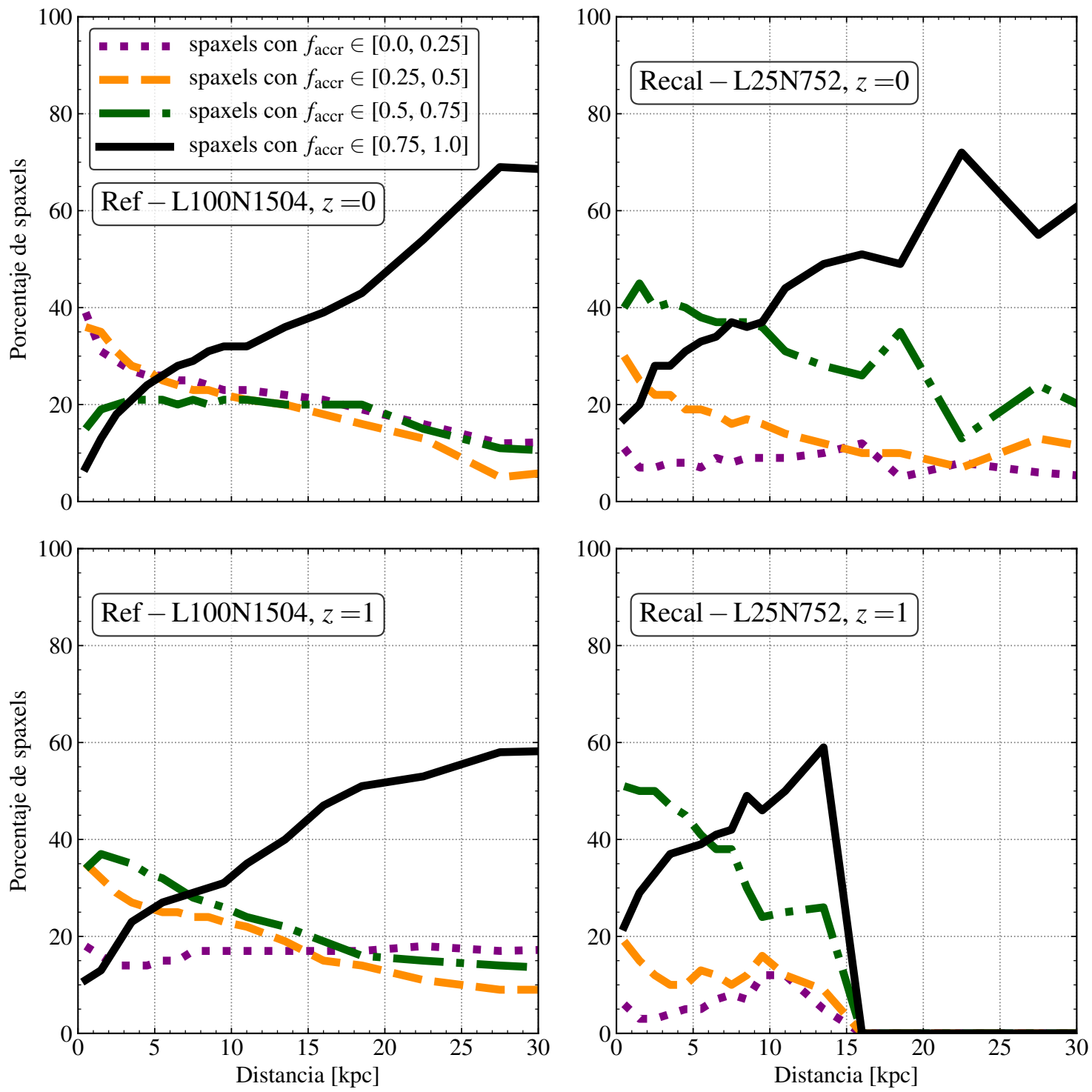

Figura 5.9: Porcentaje de spaxels en función de la distancia a $z=0$ (paneles superiores) y a $z=1$ (paneles inferiores). Los paneles izquierdos muestran los resultados de la simulación Ref-L100N1504, mientras que los paneles derechos muestran los resultados de la simulación Recal-L25N752. Las líneas violeta punteada, naranja a trazos punteada, verde a trazos y negra sólida representan los spaxels que tienen una fracción de gas acretado, $f_{\text {accr }}$, en el rango de $[0,0.25],[0.25,0.5],[0.5,0.75]$ y $[0.75,1]$, respectivamente. 

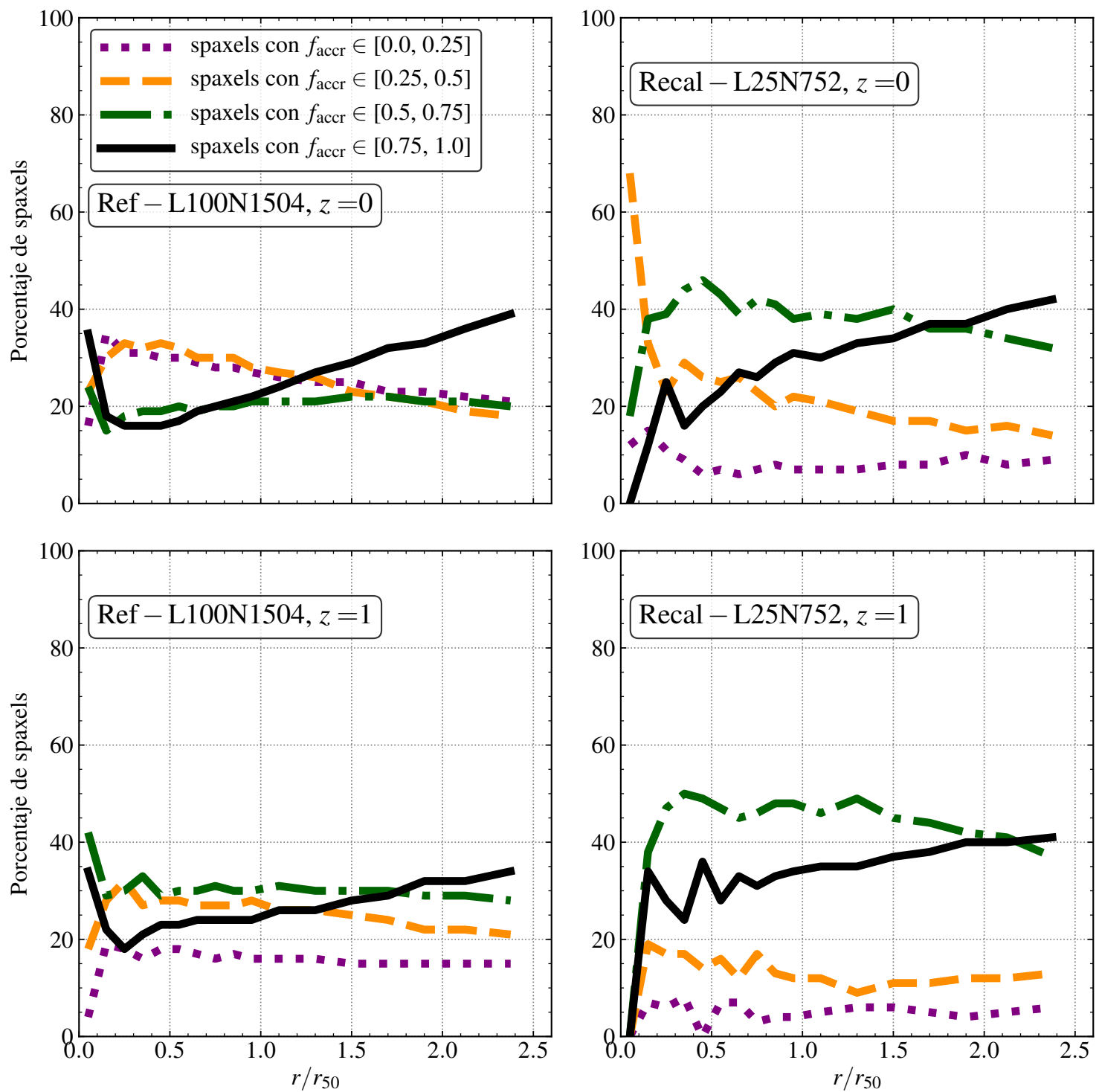

Figura 5.10: Porcentaje de spaxels en función de la distancia normalizada, $r / r_{50}$, a $z=0$ (paneles superiores) y a $z=1$ (paneles inferiores). Los paneles izquierdos muestran los resultados de la simulación Ref-L100N1504, mientras que los paneles derechos muestran los resultados de la simulación Recal-L25N752. Las líneas violeta punteada, naranja a trazos punteada, verde a trazos y negra sólida representan los spaxels que tienen una fracción de gas acretado, $f_{\text {accr }}$, en el rango de $[0,0.25]$, [0.25, 0.5], $[0.5,0.75]$ y $[0.75,1]$, respectivamente.

Los resultados para la simulación Ref-L100N1504 (paneles izquierdos) indican un comportamiento muy claro para spaxels con muy alta $f_{\text {accr }}(\geq 0.75)$ y baja $f_{\text {accr }}(\leq 0.5)$. Los primeros tienen un comportamiento creciente, es decir que a mayor distancia hay mayor cantidad de spaxels con muy alta $f_{\text {accr. }}$. En las regiones internas $(r \leq 5 \mathrm{kpc})$ sólo aportan $\lesssim 20 \%$ de la cantidad de spaxels totales a ese radio. A partir de $r \approx 15 \mathrm{kpc}$, los spaxels con muy alta $f_{\text {accr }}$ aportan más del $40 \%$ de los spaxels totales, llegando a aportar un $60-70 \%$ en las regiones más externas de las galaxias, en comparación con otros tipos de spaxels. Por otro lado, las otras clases de spaxels muestran anti-correlaciones con la distancia. Por ejemplo, los spaxels de muy baja $f_{\text {accr }}(\leq 0.25)$ predominan en las regiones internas, aportando un $30-40 \%$ de los spaxels totales hasta $r=5 \mathrm{kpc}$. Sin embargo, a medida que nos alejamos del centro de las galaxias, la cantidad de spaxels de muy baja $f_{\text {accr }}$ decrece, hasta aportar menos del $20 \%$ de los spaxels en las regiones más externas. A $z=1$, los comportamientos en general son 
similares, con la excepción que en las regiones internas predominan los spaxels con $f_{\text {accr }}$ intermedia (líneas verde punteada a trazos y naranja a trazos). Este resultado podría ser debido a que a mayor redshift las galaxias acretan mayor cantidad de gas, lo que se traduce en que en las regiones internas haya un leve incremento de esta acreción (en los PDFs de la Figura 5.6 puede verse que hay mayor cantidad de spaxels con $f_{\text {accr }}$ intermedia a $z=1$ que a $z=0$ ).
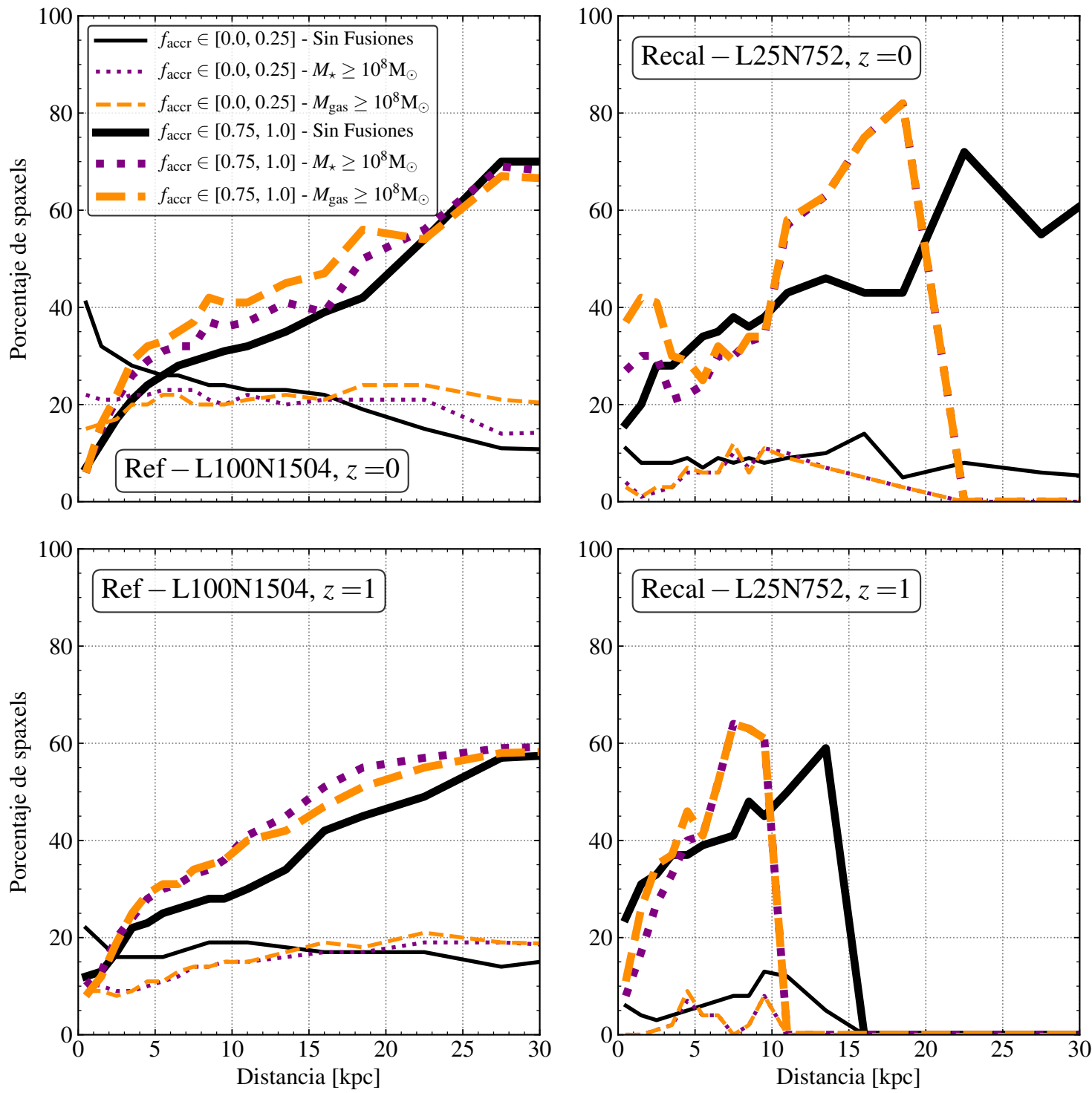

Figura 5.11: Porcentaje de spaxels en función de la distancia a $z=0$ (paneles superiores) y a $z=1$ (paneles inferiores). Los paneles izquierdos muestran los resultados de la simulación Ref-L100N1504, mientras que los paneles derechos muestran los resultados de la simulación Recal-L25N752. Las líneas negras sólidas, violetas punteadas y naranja a trazos denotan los spaxels de galaxias que no sufrieron una fusión en la salida anterior $(z=0.1)$, que han sufrido una fusión con una galaxia de masa estelar mínima de $10^{8} \mathrm{M}_{\odot}$, y que han sufrido una fusión con una galaxia con masa de gas mínima de $10^{8} \mathrm{M}_{\odot}$, respectivamente. Las líneas finas representan spaxels con muy baja fracción de gas acretado, mientras que las líneas gruesas muestran spaxels con alta fracción de gas acretado.

La simulación Recal-L25N752 (paneles derechos) muestra unas tendencias un poco diferentes. Tanto a $z=0$ como a $z=1$ los spaxels con valores muy altos de $f_{\text {accr }}$ predominan en las regiones 
externas $(r \gtrsim 10 \mathrm{kpc}$ ) con más del $40 \%$ de los spaxels. Sin embargo, en las regiones internas, los spaxels con $0.5 \leq f_{\text {accr }} \leq 0.75$ son los que predominan con $\gtrsim 40 \%$, al menos a $r \lesssim 10 \mathrm{kpc}$. A pesar de que esta relación decrece para spaxels con valores bajos de $f_{\text {accr }}$, se observa que, a radio fijo, el porcentaje de spaxels para esta $f_{\text {accr }}$ es menor en Recal-L25N752 que en Ref-L100N1504. Esto puede deberse a que hay muy poca cantidad de spaxels con valores bajos de $f_{\text {accr }}$ para la simulación Recal-L25N752 (como se observa en los paneles de los PDFs de la Figura 5.6).

Hasta aquí no hemos contemplado los tamaños de las galaxias. Estamos comparando galaxias de $10 \mathrm{kpc}$ de radio con galaxias de $20 \mathrm{kpc}$ de radio. Por lo que un radio dado podría ser el fin de una galaxia y la parte intermedia de otra. Para considerar esto, realizamos un análisis similar al de la Figura 5.9 pero graficando el porcentaje de spaxels en función de la distancia normalizada por el radio efectivo, $r_{50}$, de cada galaxia, $r / r_{50}$ (Figura 5.10). Recordemos que el radio efectivo es aquél en donde las galaxias alcanzan la mitad de su masa estelar (Sección 5.1). Nuevamente, los resultados de las simulaciones Ref-L100N1504 y Recal-L25N752 se encuentran en los paneles izquierdos y derechos, respectivamente. Los paneles superiores muestran resultados a $z=0$, mientras que los inferiores muestran resultados a $z=1$. Las clases de spaxel según su $f_{\text {accr }}$ es idéntico al de la Figura 5.9.

A grandes rasgos, los resultados que obtenemos acá son similares a los que obtuvimos recientemente: en las regiones centrales predominan los spaxels con baja $f_{\text {accr }}$ para la simulación RefL100N1504, mientras que en las regiones externas lo hacen los spaxels de alta o muy alta $f_{\text {accr }}$ (para ambas simulaciones). Las regiones internas de Recal-L25N752 tienen una mayor contribución de spaxels de alta $f_{\text {accr }}$ en comparación con Ref-L100N1504 aunque este resultado es el mismo que obtuvimos de la Figura 5.9. Por consiguiente, independientemente de la simulación que estemos analizando y el redshift, podemos extraer tres conclusiones principales de este análisis. Por un lado, tanto en las regiones internas como externas encontramos spaxels con alta $f_{\text {accr }}$, indicando que la acreción de gas puede localizarse a cualquier distancia del centro de las galaxias. Por otro lado, hay una tendencia a que haya una mayor proporción de spaxels de baja $f_{\text {accr }}$ en la región interna en comparación con la externa. Y, finalmente, hay una tendencia de que se encuentre una mayor proporción de spaxels con muy alta $f_{\text {accr }}$ en las regiones externas de las galaxias en comparación con las regiones internas, indicando así una región preferencial para la acreción de gas. La existencia de spaxels de alta $f_{\text {accr }}$ explican algunos de los resultados presentados en el Capítulo 4. En este último vimos que muchas galaxias muestran un cambio en el perfil radial de metalicidad interno, $r \leq r_{50}$, en la presencia de altas tasas de gas acretado. Acá vemos que esto se debe a que la cantidad de spaxels con alta acreción pueden ser altos incluso en las partes internas de la galaxia. La acreción de gas preferencial en las regiones externas podría estar relacionada con recientes fusiones de galaxias, que faciliten una incorporación del gas en las regiones externas mediante el procesos de fusión.

Para discernir si este camino es viable, analizamos en la Figura 5.11 la distribución de los spaxels de distinta clase de $f_{\text {accr }}$, discriminando si provienen de galaxias con una fusión reciente o no. Definimos como fusión reciente a una fusión de dos o más galaxias que sucede en la salida anterior al redshift analizado. Es decir, a $z=0$ analizamos las galaxias que son producto de dos o más galaxias hace $1.34 \mathrm{Gyr}$ (calculadas a $z=0.1$ ), mientras que al analizar las galaxias a $z=1$ calculamos su historia de fusión $0.93 \mathrm{Gyr}$ antes $(z=1.26)$. Debido a la resolución de las simulaciones, se consideran dos tipos posibles de fusiones: por un lado, fusiones producto de galaxias progenitoras con masa estelar $\geq 10^{8} \mathrm{M}_{\odot}$; por el otro, fusiones producto de galaxias progenitoras con masa de gas $\geq 10^{8} \mathrm{M}_{\odot}$ (esta última nos permite asegurarnos que las galaxias progenitoras cuenten todas con gas). Para simplificar los gráficos, nos concentramos en los spaxels de muy alta $f_{\text {accr }}(\geq 0.75$, líneas gruesas) y spaxels de muy baja $f_{\text {accr }}(\leq 0.25$, líneas finas). Las líneas negra sólida, violeta punteada y naranja a trazos representan spaxels que pertenecen a galaxias que no han sufrido fusiones recientes, a galaxias que han sufrido fusiones recientes entre galaxias de $M_{\star} \gtrsim 10^{8} \mathrm{M}_{\odot}$ y galaxias que han sufrido fusiones recientes entre galaxias con masa de gas total $\gtrsim 10^{8} \mathrm{M}_{\odot}$, respectivamente. Nuevamente, los resultados de las simulaciones Ref-L100N1504 y Recal-L25N752 se encuentran en los paneles izquierdos y 
derechos, respectivamente. Las distribuciones se muestran tanto a $z=0$ (paneles superiores), como a $z=1$ (paneles inferiores).

Las tendencias encontradas para cada simulación y cada redshift se mantienen independientemente de si las galaxias sufrieron o no una fusión reciente. Es decir, para la simulación Ref-L100N1504 predominan los spaxels de muy baja $f_{\text {accr }}$ en las regiones internas y los de muy alta $f_{\text {accr }}$ en las regiones externas, mientras que Recal-L25N752 muestra un predominio de spaxels de muy alta $f_{\text {accr }}$ a todas las distancias. Sin embargo, pueden observarse algunas diferencias entre galaxias con y sin fusiones recientes. Primero, las galaxias con fusiones recientes tienen una menor proporción de spaxels de muy baja $f_{\text {accr }}$ en las regiones internas (e incluso este comportamiento puede extenderse a regiones intermedias o externas). Segundo, las galaxias con fusiones recientes muestran una proporción de spaxels de muy alta $f_{\text {accr }}$ en las regiones internas prácticamente idéntica a la de galaxias sin fusiones. Por último, en todos los casos se observa para las galaxias con fusiones recientes un aumento considerable $(>10 \%)$ de la proporción de spaxels con muy alta $f_{\text {accr }}$ en las regiones intermedias y/o externas, en comparación con los spaxels de muy alta $f_{\text {accr }}$ de galaxias sin fusiones.

Si tenemos en cuenta los tamaños de las galaxias para este último análisis, podemos reproducir la proporción de spaxels en función, ahora, de la distancia normalizada por el radio efectivo de las galaxias, $r_{50}$, como vemos en la Figura 5.12. De esta manera, vemos un cambio en la región donde el gas es acretado. A $z=0$ hay un aumento considerable $(\gtrsim 20 \%)$ de spaxels con muy alta $f_{\text {accr }}$ en galaxias con fusiones recientes en la región de $r \lesssim 0.5 \times r_{50}$ en ambas simulaciones. RecalL25N752 muestra que, a partir de $r \approx r_{50}$, las galaxias con fusiones recientes tienen una cantidad de spaxels de muy alta $f_{\text {accr }}$ similar en relación con los spaxels de muy alta $f_{\text {accr }}$ en galaxias sin fusiones recientes. Mientras que Ref-L100N1504 muestra que las galaxias con fusiones recientes tienen $5 \%$ más de spaxels con muy alta $f_{\text {accr }}$ en comparación con galaxias $\sin$ fusiones recientes. A $z=1 \mathrm{y} \mathrm{a}$ $r \lesssim 0.5 \times r_{50}$, galaxias con fusiones recientes tienen una misma (Ref-L100N1504) o menor (RecalL25N752) cantidad de spaxels con muy alta $f_{\text {accr }}$ en comparación con galaxias sin fusiones recientes. Recal-L25N752 muestra que galaxias con fusiones recientes presentan un aumento considerable de spaxels con muy alta $f_{\text {accr }}$ cerca de $r \approx r_{50} \mathrm{y}$, a partir de esa distancia, la cantidad de estos spaxels crece con la distancia hasta superar la cantidad de spaxels de muy alta $f_{\text {accr }}$ de galaxias sin fusiones recientes.

Estos resultados indicarían que, por un lado, las fusiones de galaxias tienden a aportar mayor cantidad de gas acretado que la que reciben galaxias que no han tenido fusiones recientes, o al menos contribuyen a distribuir el gas acretado de manera diferente. Vimos que las galaxias con fusiones recientes presentan una menor cantidad de spaxels con muy baja $f_{\text {accr }}$, independientemente de si se analiza la distribución normalizada o no por $r_{50}$. Por el otro, este "extra" en la acreción pareciera depender de la masa estelar de la galaxia para definir su localización. Galaxias de la simulación RefL100N1504 (con $M_{\star} \geq 10^{10} \mathrm{M}_{\odot}$ ) que han padecido fusiones recientes muestran una mayor cantidad de spaxels con muy alta $f_{\text {accr }}$ en comparación con galaxias sin fusiones recientes. Sin embargo, galaxias con fusiones recientes de Recal-L25N752 obtienen su acreción de gas preferentemente dentro del radio efectivo con un incremento considerable en comparación con galaxias sin fusiones recientes, mientras que para $r \gtrsim r_{50}$ la cantidad de spaxels con muy alta $f_{\text {accr }}$ es igual a la cantidad de spaxels con similares valores de acreción en galaxias sin fusiones recientes $(z=0)$ o mayor a partir de $2 \times r_{50}$. Es importante en este punto tener en cuenta la incidencia de fusiones de cada simulación. Ref-L100N1504 cuenta con 116 (86) galaxias a $z=0$ que presentan fusiones recientes de galaxias con $M_{\star} \geq 10^{8} \mathrm{M}_{\odot}\left(M_{\text {gas }} \geq 10^{8} \mathrm{M}_{\odot}\right)$, mientras que Recal-L25N752 tiene 11 (8) galaxias con estas condiciones, de las cuales sólo 3 galaxias tienen $M_{\star} \leq 10^{10} \mathrm{M}_{\odot}$. A $z=1$, la situación resulta más desalentadora para Recal-L25N752, contando con 14 (9) galaxias que presentan fusiones recientes de galaxias, 2 de las cuales tienen $M_{\star} \leq 10^{10} \mathrm{M}_{\odot}$, en comparación con Ref-L100N1504 que tiene 560 (455). En este sentido, la incidencia de fusiones en galaxias de baja masa $\left(M_{\star} \leq 10^{10}\right)$ resulta mucho menor que en galaxias masivas $\left(M_{\star} \geq 10^{10}\right)$, como sugieren Lagos et al. (2018a). Esto implica que la 
estadística de los resultados de Recal-L25N752 no sea tan buena como la de Ref-L100N1504. La diferencia a distintos redshifts puede deberse a que las galaxias acretan mayor cantidad de gas a mayor redshift y para el caso particular de galaxias con fusiones recientes esto resulta acentuado. Diferenciar entre dos tipos de fusiones, una generada por la fusión de galaxias con masa estelar $\geq 10^{8} \mathrm{M}_{\odot}$ y otra generada por la fusión entre galaxias con masa de gas $\geq 10^{8} \mathrm{M}_{\odot}$, no pareciera modificar nuestras conclusiones y ambos tipos de fusiones presentarían tendencias similares.
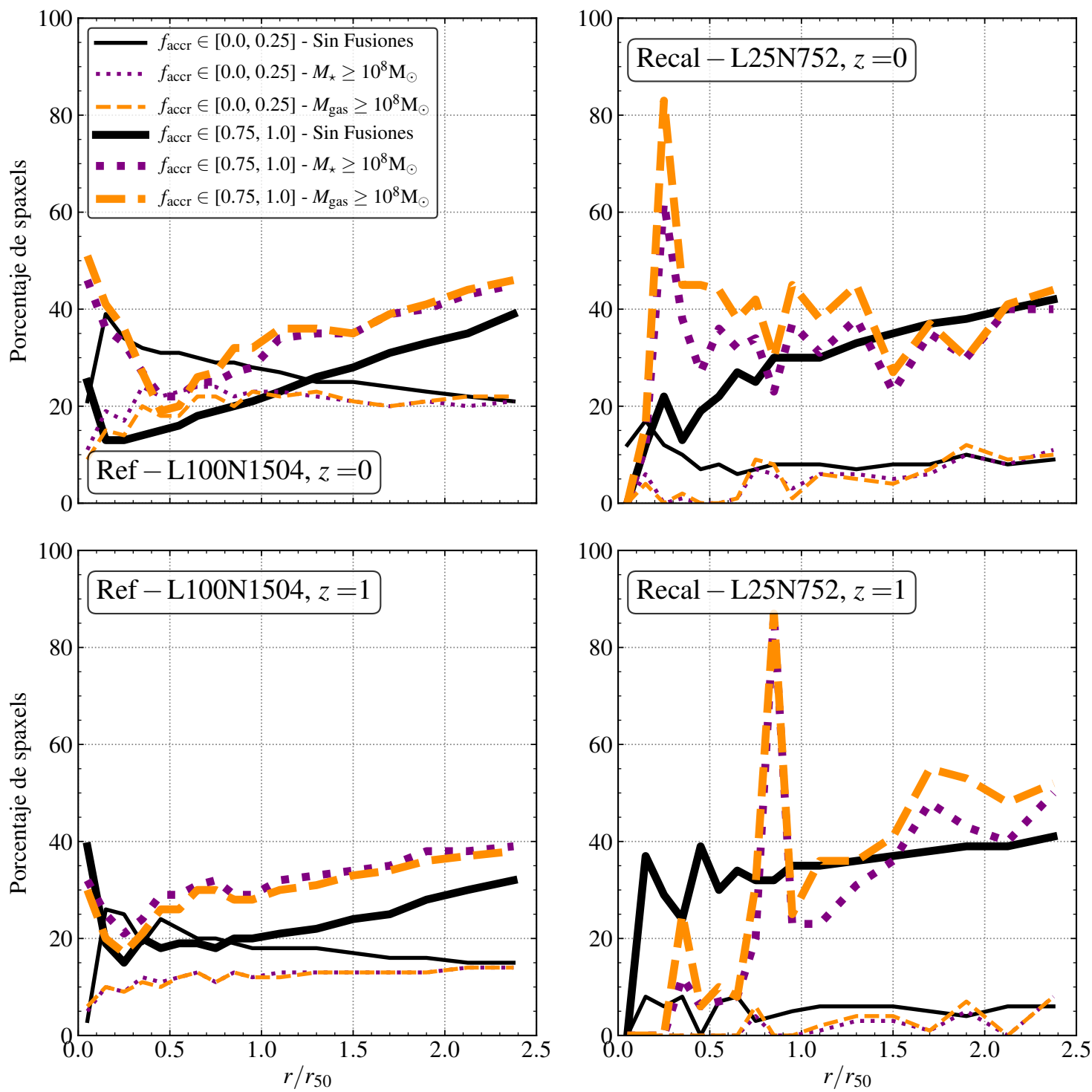

Figura 5.12: Porcentaje de spaxels en función de la distancia a $z=0$ (paneles superiores) y a $z=1$ (paneles inferiores). Los paneles izquierdos muestran los resultados de la simulación Ref-L100N1504, mientras que los paneles derechos muestran los resultados de la simulación Recal-L25N752. Las líneas negras sólidas, violetas punteadas y naranja a trazos denotan los spaxels de galaxias que no sufrieron una fusión en la salida anterior $(z=0.1)$, que han sufrido una fusión con una galaxia de masa estelar mínima de $10^{8} \mathrm{M}_{\odot}$, y que han sufrido una fusión con una galaxia con masa de gas mínima de $10^{8} \mathrm{M}_{\odot}$, respectivamente. Las líneas finas representan spaxels con muy baja fracción de gas acretado, mientras que las líneas gruesas muestran spaxels con alta fracción de gas acretado. 


\subsection{Conclusiones sobre el estudio de la metalicidad local}

En este Capítulo usamos las simulaciones del proyecto EAGLE, la simulación de referencia de mayor volumen Ref-L100N1504 y las simulaciones de alta resolución Recal-L25N752, para estudiar la metalicidad de las galaxias de manera local y su relación con otras propiedades locales, como la densidad superficial de masa estelar $\left(\Sigma_{\star}\right)$, la densidad superficial de SFR $\left(\Sigma_{\mathrm{SFR}}\right)$ y la fracción de gas acretado $\left(f_{\text {accr }}\right)$. Nos enfocamos en galaxias que tengan una masa estelar $\gtrsim 10^{10} \mathrm{M}_{\odot}$ para las simulaciones Ref-L100N1504, mientras que para Recal-L25N752 extendimos este valor hasta considerar galaxias con $M_{\star} \gtrsim 10^{9} \mathrm{M}_{\odot}$. Las galaxias simuladas analizadas aquí son centrales y con formación estelar activa ( $\mathrm{sSFR}>10^{-11} \mathrm{yr}^{-1}$ ), y tienen un redshift de $z \leq 1$.

Para encontrar las partículas de gas que están siendo acretadas por la galaxia, utilizamos un método de seguimiento, el cual sigue la historia de las partículas. Nuestro interés se enfoca en identificar una acreción de gas que desencadene formación estelar, seleccionando así aquellas partículas de gas que, en un tiempo anterior, se clasifican como no-SF. En este Capítulo presentamos los resultados del estudio de la metalicidad local de las galaxias a bajo y alto redshift, así como también analizamos la injerencia que tiene la acreción de gas a nivel local en los cambios de metalicidad observados.

Como vimos anteriormente, las simulaciones EAGLE cuentan con una combinación de volúmenes y resoluciones que no sólo permite estudiar en detalle diversas propiedades integradas de las galaxias, sino que además posibilita el estudio teórico de las regiones locales (llamadas aquí spaxels) con una estadística suficiente y sin perder confiabilidad en los resultados. Esto permite que las simulaciones EAGLE sean una herramienta más que apropiada para el estudio mostrado en este Capítulo.

A continuación, resumimos nuestras conclusiones:

- La metalicidad de los spaxels simulados crece con la $\Sigma_{\star}$, independiente de la masa estelar total de la galaxia (Figura 5.3) y del redshift (a menos hasta $z \leq 1$ ). Encontramos que las pendientes de la rMZR simulada coinciden con lo observado a $z=0$ (Figura 5.2), aunque hay una desviación en la normalización de la relación que puede explicarse debido a los diversas técnicas para inferir metalicidades en las observaciones (Ellison et al., 2008). A su vez, observamos que la $\Sigma_{\mathrm{SFR}}$ también crece con la $\Sigma_{\star}$ (Figura 5.5) en todo el rango de redshift analizado. A $z=0$, esta relación está en buen acuerdo con las observaciones (Figura 5.4). Ambas relaciones muestran tendencias similares a sus análogas relaciones globales, la MZR y la secuencia principal de galaxias.

- La metalicidad de los spaxels muestra una anti-correlación con la fracción de gas acretada, $f_{\text {accr }}$ (Figura 5.6) a redshift $z \leq 1$. La mediana de esta relación no muestra grandes signos de evolución, cambiando su normalización de $z=1 \rightarrow 0$ en sólo 0.1 dex (donde galaxias con igual $f_{\text {accr }}$ presentan mayor metalicidad a menor redshift). Esto nos está indicando que la acreción de gas diluye el contenido de metales del gas que ya se encontraba en la galaxia.

- Al comparar los residuos de la metalicidad con la $f_{\text {accr }}$ (Figura 5.7) encontramos que, a mayor valor de $f_{\text {accr }}$, la metalicidad es menor a la esperada (de hasta 0.2 dex), mientras que spaxels con menores valores de $f_{\text {accr }}$ presentan una metalicidad por encima de la esperada $(\lesssim 0.1-0.2$ dex). De forma análoga, analizamos la relación entre los residuos de metalicidad con la $\Sigma_{\mathrm{SFR}}$ (Figura 5.8), encontrando que en promedio la metalicidad no se aparta de su valor esperado al variar la $\Sigma_{\mathrm{SFR}}$. Esto nos indica que la metalicidad es más sensible a la $f_{\text {accr }}$ que a otras propiedades locales como la $\Sigma_{\mathrm{SFR}}$. Así, la metalicidad inferida observacionalmente podría ser una buena indicadora de regiones donde se produjo una reciente acreción de gas. Estos resultados se mantienen en todo el rango de redshift analizado.

- Las regiones internas de las galaxias $\left(\lesssim 0.5 \times r_{50}\right)$ muestran una mayor cantidad de spaxels con $f_{\text {accr }}$ muy baja $(\leq 0.25)$ en comparación con spaxels con otros valores de $f_{\text {accr }}$ (Figura 5.10). 
A su vez, a medida que tomamos spaxels cada vez más lejanos a los centros de las galaxias $\left(\gtrsim 1 \times r_{50}\right)$ comienza a haber mayor proporción de spaxels con muy alta $f_{\text {accr }}(\geq 0.75)$ que spaxels con menores valores de $f_{\text {accr }}$. Esto nos indica que los spaxels con menor metalicidad se van a alojar, preferentemente, en las regiones más externas de las galaxias, dado que los spaxels con mayor $f_{\text {accr }}$ tienen menor metalicidad.

- Hay una tendencia de que galaxias que han sufrido fusiones recientes tengan una mayor cantidad de spaxels con valores de $f_{\text {accr }}$ muy altos, en particular en las regiones centrales, es decir $r \lesssim r_{50}$ (Figura 5.12).

Este estudio es pionero en el análisis del efecto de la acreción de gas en la metalicidad de las regiones resueltas o locales de galaxias, desde el punto de vista teórico. En el mismo, mostramos que la acreción de gas modifica directamente el contenido químico del gas de las galaxias, en comparación con otras propiedades, como por ejemplo, la $\Sigma_{\mathrm{SFR}}$. Desde un punto de vista local, esto significa que la formación estelar no determina la metalicidad como se deduce de los resultados a partir de propiedades integradas (al menos, a primer orden), sino que es la acreción de gas lo que determina el grado de enriquecimiento químico que una región galáctica tiene. Esto llevaría a pensar que la acreción de gas resulta más fundamental a nivel local para determinar los cambios en la metalicidad que otras propiedades de galaxias. Algo interesante de analizar a futuro es el comportamiento de galaxias satélites, las cuales tienen una supresión de la acreción de gas, investigando cómo se modifican las relaciones aquí estudiadas; este enfoque sería interesante para aportar más información a los resultados presentados en este Capítulo.

Observacionalmente, inferir la acreción de gas resulta un desafío. De esta manera, no sólo confirmamos que la metalicidad es una buena estimadora de la acreción de gas, sino que además los residuos de la metalicidad dan información relevante sobre esta acreción. Los residuos tienen la ventaja de no estar tan afectados por el calibrador de metalicidad utilizado como la metalicidad en sí, por lo que resulta más directa la comparación con diversos estudios.

Un camino a seguir a partir de aquí es analizar si estos resultados se mantienen para otros elementos químicos, como el hierro, de forma de poder discernir si otros elementos resultan tan sensibles a la acreción de gas como el oxígeno. Tanto una respuesta afirmativa como negativa tienen importante interés. De cumplirse, hay otras posibilidades para poder inferir la acreción de gas de manera observacional. En caso contrario, distintos elementos químicos podrían estar relacionados a distintos procesos, lo que llevaría a realizar diferentes análisis dependiendo del proceso físico que se quiera estudiar. 


\section{Capítulo 6}

\section{Conclusiones}

Esta Tesis tiene como objetivo estudiar la evolución química de las galaxias con el fin de mejorar nuestro entendimiento sobre la formación de éstas, al estudiar la relación entre diversas propiedades de las galaxias, entre ellas, la metalicidad de la fase fría del gas, y los procesos físicos que determinan las mismas. Para ello, se profundiza en el estudio de la relación masa-metalicidad de las galaxias. La misma puede dar indicios sobre los diferenes procesos de acreción y eyección de gas, a la vez que refleja los proccesos de feedback (entre otros) que las galaxias han sufrido a lo largo del tiempo. A su vez, se analiza la composición química de las galaxias a lo largo de sus discos con el fin de dar aportes sobre las propiedades que moldean la forma de los perfiles radiales de metalicidad, mientras que se analiza si hay alguna región preferencial donde la metalicidad es considerablemente alta o baja.

El estudio del enriquecimiento químico de las galaxias presentado aquí se ha realizado utilizando tanto el modelo semi-analítico de formación y evolución de galaxias SAG como las simulaciones hidrodinámicas cosmológicas EAGLE (descritos en el Capítulo 2). Dado que el estudio de la formación de galaxias carece de un tratamiento experimental debido a que es imposible rehacer un Universo entero, el uso de simulaciones numéricas cosmológicas permite simular porciones del Universo con el fin de entender los procesos físicos involucrados en la formación de las galaxias. La versatilidad que tienen estas simulaciones numéricas para simular bloques representativos del Universo utilizando diversas aproximaciones las convierten en excelentes laboratorios cosmológicos. En particular, el modelo semi-analítico SAG cuenta con una descripción del enriquecimiento químico no instantánea (entre muchos otros procesos), y es corrido sobre una simulación de DM de $1 \mathrm{Gpc} h^{-1}$ de lado. Esto permite generar una gran población de galaxias en ambientes de distina densidad, es decir, tanto galaxias aisladas como galaxias residentes en grandes cúmulos de galaxias. Por su parte, las simulaciones hidrodinámicas EAGLE son simulaciones de punta las cuales generan una población de galaxias lo suficientemente grande como para tener una buena estadística, al tiempo que pueden obtenerse características tanto globales como locales al contar con galaxias simuladas que son espacialmente resueltas (a diferencia de SAG y, en general, de los modelos semi-analíticos). A pesar de que las simulaciones numéricas presentan diferentes desventajas según sean modelos semi-analíticos o simualciones hidrodinámicas autoconsistentes, tales como limitaciones en la resolución de masa o resolución espacial, incapacidad para generar galaxias como objetos extendidos, y la carencia de una modelización matemática conocida y/o consensuada de ciertos procesos físicos (entre otras), el estudio teórico de la formación de las galaxias resulta clave para poder desentrañar posibles caminos evolutivos de las galaxias, mientras se encuentran resultados convergentes utilizando distintos métodos.

En primera instancia, analizamos las predicciones de cuatro versiones del modelo semi-analítico SAG sobre la evolución de la relación masa-metalicidad en el Capítulo 3. Estas versiones varían, principalmente, en la prescripción del modelado del feedback de supernovas (modelos $\mathrm{SAG}_{\beta 1.9}, \mathrm{SAG}_{\beta 1.3}$ y $\mathrm{SAG}_{\beta 0.0}$ ) y en la receta que determina el destino del gas reciclado $\left(\mathrm{SAG}_{\beta 1.9}\right.$ y $\left.\mathrm{SAG}_{\beta 1.9-\mathrm{Rec}}\right)$, como 
se detalla en la Sección 2.1. Para que SAG pueda reproducir la evolución observada de la MZR, que indica que galaxias de masa estelar similar presentan metalicidades más bajas a mayor redshift, es necesario un feedback de SN suficientemente eficiente a alto redshift, y que esa eficiencia decrezca con el tiempo. Así, la eficiencia de los vientos generados por las SNs resulta mayor a alto redshift, lo que produce una inyecta de energía al medio tal que transporta los metales recientemente sintetizados y aquéllos ya presentes en el gas directamente a la fase gaseosa caliente, retrasando la contaminación del gas frío en donde las estrellas se forman. Entonces, a medida que pasa el tiempo (y gracias al enfriamiento radiativo) el gas frío se contamina gradualmente, lo que lleva a que la MZR evolucione. En este sentido, el feedback de SN resulta un regulador de la contaminación del gas, no sólo por ser responsable de inyectar metales al ISM (tanto los metales que se encontraban en la estrella al formarse como los metales recientemente sintetizados), sino por ser el principal encargado de definir la fase gaseosa donde esos metales se depositan.

En segundo lugar, mostramos la distribución de metales teniendo en cuenta su distancia con respecto al centro de la galaxia en la que se encuentran, es decir, analizando los perfiles radiales de metalicidad. Para ello, es necesario describir las galaxias como objetos extendidos, por lo que hacemos uso de las simulaciones EAGLE. Así, no sólo analizamos propiedades globales de las galaxias (como la acreción de gas, la masa estelar y la metalicidad), sino que descomponemos a las galaxias en anillos concéntricos y calculamos la metalicidad producida en cada anillo. Sin embargo, analizamos los perfiles sólo hasta un radio efectivo $\left(r_{50}\right)$, donde encontramos que hay mayores cambios. Para poder cuantificar estos cambios, calculamos la pendiente interna de los RMP de las galaxias, $\alpha$, utilizando un ajuste lineal. Así, analizamos los cambios que esta pendiente $\alpha$ muestra en función de la masa estelar de las galaxias, la tasa de acreción de gas $\left(\dot{M}_{\text {accr }}\right)$, la SFR y la fracción de gas $\left(f_{\text {gas }}\right)$. Encontramos que $\alpha$ decrece (se hace más negativa) a medida que estas propiedades aumentan, aunque significativamente de manera distinta para cada propiedad, siendo mayor el cambio para $\dot{M}_{\text {accr }}$. Es decir, galaxias que presentan valores altos de $\dot{M}_{\text {accr }}$ también muestran valores más negativos de $\alpha$, lo que lleva a RMP internos más empinados, mientras que galaxias con valores bajos de $\dot{M}_{\text {accr }}$ presentan RMP internos más planos. Debido a que la masa estelar está relacionada con las otras propiedades mencionadas, decidimos eliminar esa dependencia para corroborar si las relaciones encontradas se siguien manteniendo. De esta manera, procedimos al cálculo de residuos, en donde se obtiene la diferencia de una propiedad respecto de los valores medianos de la relación entre esa propiedad y la masa estelar (como se detalla en la Sección 4.5.2). Así, encontramos que los residuos de $\alpha$ siguen mostrando una clara anti-correlación con los residuos de la $\dot{M}_{\text {accr }}$. Esto nos indica que la tasa de acreción de gas juega un papel importante en la regulación de la distribución de los metales en las galaxias. Cuanto mayor es la tasa de acreción de gas, más rápida y eficiente será la formación estelar y, por lo tanto, la liberación de metales y energía al medio.

Por último, en el Capítulo 5 nos adentramos en el estudio de las propiedades de las galaxias localmente resueltas. Es decir, en lugar de estudiar las propiedades globales de éstas (como la masa estelar, la SFR o la masa de gas total), dividimos las galaxias en regiones resueltas (con tamaños de $1 \mathrm{kpc}$ $\times 1 \mathrm{kpc}$ ) y estudiamos sus propiedades; nos referimos a estas regiones como spaxels. Así, estudiamos la metalicidad de los spaxels y las relaciones que tienen con otras propiedades resueltas, como la densidad superficial de masa estelar, $\Sigma_{\star}$, la densidad superficial de SFR, $\Sigma_{\mathrm{SFR}}$, y la fracción de gas acretado, $f_{\text {accr }}$. Encontramos que la metalicidad de los spaxels crece al aumentar tanto $\Sigma_{\star}$ como $\Sigma_{\mathrm{SFR}}$, de manera análoga a lo que se deduce de la MZR y de la secuencia principal de galaxias. Además, encontramos que al aumentar la $f_{\text {accr }}$ la metalicidad local disminuye, hecho que sucede independientemente de la masa estelar total de la galaxia, de la simulación utilizada, o incluso del redshift. Esta relación resulta incluso más fuerte que la hallada para la relación entre la metalicidad local y la $\Sigma_{\mathrm{SFR}}$. A su vez, vimos que los spaxels cuya $f_{\text {accr }}$ es relativamente alta $(\geq 0.75)$ se encuentran preferencialmente en las regiones externas de las galaxias, mientras que el grueso de los spaxels con valores bajos de $f_{\text {accr }}(\leq 0.25)$ predominan en las regiones internas. De estos resultados se desprende que la 
metalicidad es significativamente sensible a la acreción de gas y entonces puede ser una buena estimadora de la acreción en trabajos observacionales. También se concluye que esta acreción se produce predominantemente en las regiones externas, donde los spaxels que allí se encuentran resultan ser de menor metalicidad que en las regiones internas (lo que genera un RMP negativo). Estos resultados se mantienen cuando se distingue las galaxias que han sufrido fusiones recientes de las que no, con la única diferencia que las primeras cuentan con una mayor cantidad de spaxels con $f_{\text {accr }}$ alta $(>0.75)$ en comparación con las segundas.

Ciertamiente, estos resultados son novedosos e importantes para el estudio del enriquecimiento químico de las galaxias, sin embargo, todavía queda mucho por hacer. Por un lado, no encontramos un cambio significativo en las pendientes de la MZR a distintos $z$ al estudiarlas con el modelo SAG. Además, la MZR modelada por SAG no presenta un aplanamiento para galaxias de alta masa $\left(M_{\star} \gtrsim 10^{11} \mathrm{M}_{\odot}\right)$, lo cual sí se observa tanto en observaciones como en simulaciones hidródinámicas (como por ejemplo EAGLE; S15). Esto puede estar relacionado a que el modelado del feedback de AGN actualmente implementado no es lo suficientemente efectivo a distintos redshifts como para que se aprecie ese cambio en la relación. Actualmente, se está trabajando en la implementación de un modelo de feedback de AGN que capture más cabalmente la complejidad del fenómeno, por lo que podríamos encontrar nuevos resultados una vez que el nuevo modelo se encuentre calibrado. Asimismo, se puede incorporar una descripción de la distribución espacial de las componentes bariónicas de las galaxias en los SAMs dando lugar a la generación de perfiles radiales de distintas propiedades de las galaxias (Henriques et al., 2020; Xie et al., 2020). Aunque las aproximaciones utilizadas en este tipo de implementaciones se sumen a las ya existentes, la disponibilidad de un modelo con estas características puede ser muy útil para entender cómo se establecen los perfiles radiales de distintas propiedades, en particular, los RMPs. Así, se propone realizar modificaciones al modelo SAG con el fin de incorporar una descripción de los RMPs de las galaxias.

Por otro lado, al estudiar los RMPs que se extraen de las simulaciones EAGLE encontramos que las regiones externas no resultan bien definidas (debido a que el número de partículas cae considerablemente), además de que la $\dot{M}_{\text {accr }}$ se obtiene a partir de un cálculo simple y, entonces, resulta una cota inferior. En esta línea, extender el estudio de los RMPs utilizando un cálculo más refinado de la $\dot{M}_{\text {accr }}$ sería interesante para poder delimitar si las distintas fuentes de esta acreción alteran significativamente la forma de los perfiles. Además, incorporar el estudio de los RMPs de las galaxias satélites aportaría información relevante. Así, se espera que el ambiente en donde las galaxias están inmersas tenga un efecto importante sobre la acreción de material que las galaxias padecen y, entonces, el grado de diferencia que encontremos con los resultados aquí expuestos puede permitir identificar distintas fuentes de acreción de gas o, incluso, procesos que evitan dicha acreción.

Asimismo, el estudio de los RMPs aquí presentado no tiene en cuenta la simetría axial de las galaxias. Para abordar esto (al menos a primer orden), se analizaron las propiedades de los spaxels de galaxias. Sin embargo, al igual que con el estudio anterior sólo se analizaron los spaxels de galaxias centrales. Extender el estudio de las propiedades locales a galaxias satélites resulta de gran interés, dado que éstas están fuertemente afectadas por el ambiente en que se encuentran. Además, analizar si nuestros resultados varían dependiendo del elemento químico utilizado para determinar la metalicidad (recordemos que aquí se analizó la metalicidad como la abundacia del oxígeno relativa al hidrógeno) podría permitir encontrar distintos mecanismos de acreción dependiendo del elemento químico o, en caso contrario, ampliar la búsqueda de la acreción de gas de manera observacional al utilizar otros indicadores, además del oxígeno.

Gracias a las nuevas generaciones de simulaciones, como CLUSTER-EAGLE (Barnes et al., 2017), EAGLE-XL, COLIBRE o las simulaciones Hydrangea (Bahé et al., 2017), así como también a los nuevos relevamientos observacionales (como MAGPI), un estudio del enriquecimiento químico de las galaxias teniendo en cuenta el ambiente en donde estas se encuentran resulta el paso siguiente natural en estudio de la evolución química de las galaxias. 


\section{Bibliografía}

Anders, E. \& Grevesse, N. 1989, Geochim. Cosmochim. Acta, 53, 197

Andrews, B. H. \& Martini, P. 2013, ApJ, 765, 140

Anglés-Alcázar, D. et al. 2017, MNRAS, 470, 4698

Arnold, C., Leo, M. \& Li, B. 2019, Nature Astronomy, 3, 945

Asplund, M., Grevesse, N. \& Sauval, A. J. 2005, in Astronomical Society of the Pacific Conference Series, Vol. 336, Cosmic Abundances as Records of Stellar Evolution and Nucleosynthesis, ed. T. G. Barnes, III \& F. N. Bash, 25

Baes, M. et al. 2011, ApJS, 196, 22

Bahé, Y. M. et al. 2017, MNRAS, 470, 4186

Bahé, Y. M. et al. 2016, MNRAS, 456, 1115

Baldry, I. K. et al. 2012, MNRAS, 421, 621

Barnes, D. J. et al. 2017, MNRAS, 471, 1088

Barrera-Ballesteros, J. K. et al. 2018, ApJ, 852, 74

Barrera-Ballesteros, J. K. et al. 2016, MNRAS, 463, 2513

Baugh, C. M. 2006, Reports on Progress in Physics, 69, 3101

Behroozi, P. S., Wechsler, R. H. \& Conroy, C. 2013a, ApJ, 770, 57

Behroozi, P. S., Wechsler, R. H. \& Wu, H.-Y. 2013b, ApJ, 762, 109

Behroozi, P. S. et al. 2013c, ApJ, 763, 18

Belfiore, F. et al. 2017, MNRAS, 469, 151

Bennett, C. L. et al. 2013, ApJS, 208, 20

Benson, A. J. 2010, Phys. Rep., 495, 33

Bernardi, M. et al. 2017, MNRAS, 467, 2217

Blumenthal, G. R. et al. 1984, Nature, 311, 517

Bode, P., Ostriker, J. P. \& Turok, N. 2001, ApJ, 556, 93

Boissier, S. \& Prantzos, N. 1999, MNRAS, 307, 857 
Booth, C. M. \& Schaye, J. 2010, MNRAS, 405, L1

Booth, R. S. et al. 2009, arXiv e-prints, arXiv:0910.2935

Boselli, A. et al. 2014, A\&A, 564, A66

Bothwell, M. S. et al. 2013, MNRAS, 433, 1425

Bouché, N. F. 2017, Astrophysics and Space Science Library, Vol. 430, Gas Accretion and StarFormation Rates with IFUs and Background Quasars, ed. A. Fox \& R. Davé, 355

Bower, R. G. et al. 2006, MNRAS, 370, 645

Bower, R. G. et al. 2017, MNRAS, 465, 32

Brown, T. et al. 2017, MNRAS, 466, 1275

Brown, T. et al. 2018, MNRAS, 473, 1868

Bryant, J. J. et al. 2015, MNRAS, 447, 2857

Bundy, K. et al. 2015, ApJ, 798, 7

Bunn, E. F. \& White, M. 1997, ApJ, 480, 6

Cano-Díaz, M. et al. 2016, ApJ, 821, L26

Carton, D. et al. 2018, MNRAS, 478, 4293

Carton, D. et al. 2015, MNRAS, 451, 210

Chabrier, G. 2003, PASP, 115, 763

Chen, Y.-M. et al. 2012, MNRAS, 421, 314

Cole, S. et al. 2000, MNRAS, 319, 168

Collacchioni, F. et al. 2018, MNRAS, 481, 954

Collacchioni, F. et al. 2021, Boletin de la Asociacion Argentina de Astronomia La Plata Argentina, 62,165

Collacchioni, F. et al. 2020, MNRAS, 495, 2827

Cora, S. A. 2006, MNRAS, 368, 1540

Cora, S. A. et al. 2019, MNRAS, 483, 1686

Cora, S. A. et al. 2018, MNRAS, 479, 2

Correa, C. A. et al. 2018, MNRAS, 478, 255

Crain, R. A. et al. 2017, MNRAS, 464, 4204

Crain, R. A. et al. 2015, MNRAS, 450, 1937

Creasey, P., Theuns, T. \& Bower, R. G. 2013, MNRAS, 429, 1922

Cresci, G. et al. 2010, Nature, 467, 811 
Croton, D. J. et al. 2006, MNRAS, 365, 11

Dalla Vecchia, C. \& Schaye, J. 2012, MNRAS, 426, 140

Davé, R., Finlator, K. \& Oppenheimer, B. D. 2011, MNRAS, 416, 1354

Davé, R., Finlator, K. \& Oppenheimer, B. D. 2012, MNRAS, 421, 98

Davé, R. et al. 2017, MNRAS, 467, 115

Davis, M. et al. 1985, ApJ, 292, 371

De Rossi, M. E. et al. 2017, MNRAS, 472, 3354

Díaz, Á. I. et al. 2007, MNRAS, 382, 251

Dolag, K. et al. 2009, MNRAS, 399, 497

Driver, S. P. et al. 2018, MNRAS, 475, 2891

Einasto, J. 1965, Trudy Astrofizicheskogo Instituta Alma-Ata, 5, 87

Eke, V. R., Cole, S. \& Frenk, C. S. 1996, MNRAS, 282, 263

Ellis, R. 2000, in Astronomical Society of the Pacific Conference Series, Vol. 195, Imaging the Universe in Three Dimensions, ed. W. van Breugel \& J. Bland-Hawthorn, 13

Ellison, S. L. et al. 2008, ApJ, 672, L107

Erb, D. K. et al. 2006, ApJ, 644, 813

Erroz-Ferrer, S. et al. 2019, MNRAS, 484, 5009

Ferland, G. J. et al. 1998, PASP, 110, 761

Finlator, K. 2017, in Astrophysics and Space Science Library, Vol. 430, Gas Accretion onto Galaxies, ed. A. Fox \& R. Davé, 221

Foster, A. R. et al. 2012, ApJ, 756, 128

Foster, C. et al. 2020, arXiv e-prints, arXiv:2011.13567

Fox, A. \& Davé, R., eds. 2017, Astrophysics and Space Science Library, Vol. 430, Gas Accretion onto Galaxies

Furlong, M. et al. 2017, MNRAS, 465, 722

Furlong, M. et al. 2015, MNRAS, 450, 4486

Gargiulo, I. D. et al. 2015, MNRAS, 446, 3820

Gingold, R. A. \& Monaghan, J. J. 1977, MNRAS, 181, 375

Gonzalez-Perez, V. et al. 2014, MNRAS, 439, 264

Grevesse, N. \& Sauval, A. J. 1998, Space Sci. Rev., 85, 161

Gruppioni, C. et al. 2015, MNRAS, 451, 3419 
Gunn, J. E. \& Gott, J. R. I. 1972, ApJ, 176, 1

Guo, Q. et al. 2016, MNRAS, 461, 3457

Guo, Q. et al. 2011, MNRAS, 413, 101

Haardt, F. \& Madau, P. 2001, in Clusters of Galaxies and the High Redshift Universe Observed in X-rays, ed. D. M. Neumann \& J. T. V. Tran, 64

Hägele, G. F. 2008, PhD thesis, Universidad Autónoma de Madrid

Heald, G. et al. 2011, A\&A, 526, A118

Henriques, B. M. B. et al. 2009, MNRAS, 396, 535

Henriques, B. M. B. et al. 2015, MNRAS, 451, 2663

Henriques, B. M. B. et al. 2013, MNRAS, 431, 3373

Henriques, B. M. B. et al. 2020, MNRAS, 491, 5795

Hirschmann, M., De Lucia, G. \& Fontanot, F. 2016, MNRAS, 461, 1760

Ho, I. T. et al. 2015, MNRAS, 448, 2030

Ho, S. H., Martin, C. L. \& Turner, M. L. 2019, ApJ, 875, 54

Hopkins, P. F. 2013, MNRAS, 428, 2840

Hopkins, P. F. et al. 2014, MNRAS, 445, 581

Hopkins, P. F., Quataert, E. \& Murray, N. 2012, MNRAS, 421, 3522

Hsieh, B. C. et al. 2017, ApJ, 851, L24

Hubble, E. P. 1929, ApJ, 69, 103

Hughes, T. M. et al. 2013, A\&A, 550, A115

Hwang, H.-C. et al. 2019, ApJ, 872, 144

Jiang, L. et al. 2014, MNRAS, 440, 2115

Johnston, S. et al. 2008, Experimental Astronomy, 22, 151

Kacprzak, G. G. 2017, Astrophysics and Space Science Library, Vol. 430, Gas Accretion in StarForming Galaxies, ed. A. Fox \& R. Davé, 145

Kamphuis, P. et al. 2013, MNRAS, 434, 2069

Kant, I. 1755, Allgemeine Naturgeschichte und Theorie des Himmels

Kennicutt, Robert C., J. 1998, ApJ, 498, 541

Kewley, L. J. \& Ellison, S. L. 2008, ApJ, 681, 1183

Klypin, A. et al. 2016, MNRAS, 457, 4340

Knebe, A. et al. 2018, MNRAS, 474, 5206 
Kormendy, J. \& Ho, L. C. 2013, ARA\&A, 51, 511

Kroupa, P. 2001, MNRAS, 322, 231

Lacey, C. G. et al. 2016, MNRAS, 462, 3854

Lagos, C. D. P. et al. 2014, MNRAS, 440, 920

Lagos, C. d. P. et al. 2012, MNRAS, 426, 2142

Lagos, C. D. P., Cora, S. A. \& Padilla, N. D. 2008, MNRAS, 388, 587

Lagos, C. d. P. et al. 2015, MNRAS, 452, 3815

Lagos, C. d. P., Lacey, C. G. \& Baugh, C. M. 2013, MNRAS, 436, 1787

Lagos, C. d. P. et al. 2018a, MNRAS, 473, 4956

Lagos, C. d. P. et al. 2016, MNRAS, 459, 2632

Lagos, C. d. P. et al. 2018b, MNRAS, 481, 3573

Lange, R. et al. 2016, MNRAS, 462, 1470

Lara-López, M. A. et al. 2010, A\&A, 521, L53

Lara-López, M. A. et al. 2013, MNRAS, 433, L35

Le Brun, A. M. C. et al. 2014, MNRAS, 441, 1270

Lequeux, J. et al. 1979, A\&A, 500, 145

Leroy, A. K. et al. 2008, AJ, 136, 2782

Leslie, S. K. et al. 2020, ApJ, 899, 58

Li, C. \& White, S. D. M. 2009, MNRAS, 398, 2177

Lilly, S. J. et al. 2013, ApJ, 772, 119

Lucy, L. B. 1977, AJ, 82, 1013

Ludlow, A. D., Schaye, J. \& Bower, R. 2019a, MNRAS, 488, 3663

Ludlow, A. D. et al. 2019b, MNRAS, 488, L123

Ly, C. et al. 2016, ApJ, 828, 67

Ma, X. et al. 2016, MNRAS, 456, 2140

Ma, X. et al. 2017, MNRAS, 466, 4780

Macciò, A. V. et al. 2019, MNRAS, 484, 5400

Maiolino, R. \& Mannucci, F. 2019, A\&A Rev., 27, 3

Maiolino, R. et al. 2008, A\&A, 488, 463

Mannucci, F. et al. 2010, MNRAS, 408, 2115 
Mannucci, F. et al. 2009, MNRAS, 398, 1915

Marigo, P. 2001, A\&A, 370, 194

Marino, R. A. et al. 2016, A\&A, 585, A47

Matthee, J. \& Schaye, J. 2018, MNRAS, 479, L34

McAlpine, K. et al. 2015, Advancing Astrophysics with the Square Kilometre Array (AASKA14), 83

McAlpine, S. et al. 2017, MNRAS, 468, 3395

McAlpine, S. et al. 2016, Astronomy and Computing, 15, 72

McCarthy, I. G. et al. 2011, MNRAS, 412, 1965

McClure, R. D. \& van den Bergh, S. 1968, AJ, 73, 1008

McConnell, N. J. \& Ma, C.-P. 2013, ApJ, 764, 184

Mendel, J. T. et al. 2014, ApJS, 210, 3

Mitchell, P. D., Schaye, J. \& Bower, R. G. 2020, MNRAS, 497, 4495

Mo, H., van den Bosch, F. C. \& White, S. 2010, Galaxy Formation and Evolution

Mo, H. J., Mao, S. \& White, S. D. M. 1998, MNRAS, 295, 319

Mollá, M. et al. 2016, MNRAS, 462, 1329

Møller, P. et al. 2013, MNRAS, 430, 2680

Monaghan, J. J. 1992, ARA\&A, 30, 543

Montuori, M. et al. 2010, A\&A, 518, A56

Moster, B. P., Naab, T. \& White, S. D. M. 2013, MNRAS, 428, 3121

Muñoz Arancibia, A. M. et al. 2015, MNRAS, 446, 2291

Muratov, A. L. et al. 2015, MNRAS, 454, 2691

Muratov, A. L. et al. 2017, MNRAS, 468, 4170

Naab, T. et al. 2014, MNRAS, 444, 3357

Naab, T. \& Ostriker, J. P. 2017, ARA\&A, 55, 59

Nagao, T., Maiolino, R. \& Grazian, A. 2008, in Astronomical Society of the Pacific Conference Series, Vol. 399, Panoramic Views of Galaxy Formation and Evolution, ed. T. Kodama, T. Yamada, \& K. Aoki, 272

Navarro, J. F., Frenk, C. S. \& White, S. D. M. 1997, ApJ, 490, 493

Neistein, E. et al. 2012, MNRAS, 421, 3579

Nelson, D. et al. 2019, MNRAS, 490, 3234

Nelson, D. et al. 2013, MNRAS, 429, 3353 
Oosterloo, T. A. et al. 2007, A\&A, 465, 787

Padilla, N. D. et al. 2014, MNRAS, 443, 2801

Padovani, P. \& Matteucci, F. 1993, ApJ, 416, 26

Pagel, B. E. J. et al. 1979, MNRAS, 189, 95

Patrício, V. et al. 2019, MNRAS, 489, 224

Peacock, J. A. 1997, MNRAS, 284, 885

Perez, J., Michel-Dansac, L. \& Tissera, P. B. 2011, MNRAS, 417, 580

Pilyugin, L. S. et al. 2017, A\&A, 608, A127

Planck Collaboration et al. 2014a, A\&A, 571, A1

Planck Collaboration et al. 2014b, A\&A, 571, A16

Planck Collaboration et al. 2020, A\&A, 641, A6

Poetrodjojo, H. et al. 2018, MNRAS, 479, 5235

Popping, A. et al. 2009, A\&A, 504, 15

Portinari, L., Chiosi, C. \& Bressan, A. 1998, A\&A, 334, 505

Press, W. H. \& Schechter, P. 1974, ApJ, 187, 425

Puchwein, E. \& Springel, V. 2013, MNRAS, 428, 2966

Putman, M. E. 2017, Astrophysics and Space Science Library, Vol. 430, An Introduction to Gas Accretion onto Galaxies, ed. A. Fox \& R. Davé, 1

Qu, Y. et al. 2017, MNRAS, 464, 1659

Rahmati, A. et al. 2013a, MNRAS, 430, 2427

Rahmati, A. et al. 2013b, MNRAS, 431, 2261

Riess, A. G. et al. 1998, AJ, 116, 1009

Rosa, D. A. et al. 2014, MNRAS, 444, 2005

Rosales-Ortega, F. F. et al. 2010, MNRAS, 405, 735

Rosales-Ortega, F. F. et al. 2012, ApJ, 756, L31

Ruiz, A. N. et al. 2015, ApJ, 801, 139

Rupke, D. S. N., Veilleux, S. \& Baker, A. J. 2008, ApJ, 674, 172

Salmon, J. 1996, ApJ, 460, 59

Sánchez, S. F. et al. 2017, MNRAS, 469, 2121

Sánchez, S. F. et al. 2012, A\&A, 538, A8

Sánchez, S. F. et al. 2014, A\&A, 563, A49 
Sánchez, S. F. et al. 2013, A\&A, 554, A58

Sánchez Almeida, J. et al. 2018, MNRAS, 476, 4765

Sánchez Almeida, J. et al. 2015, ApJ, 810, L15

Sánchez-Menguiano, L. et al. 2016, A\&A, 587, A70

Sánchez-Menguiano, L. et al. 2019, ApJ, 882, 9

Savaglio, S. et al. 2005, in American Institute of Physics Conference Series, Vol. 761, The Spectral Energy Distributions of Gas-Rich Galaxies: Confronting Models with Data, ed. C. C. Popescu \& R. J. Tuffs, 425-428

Schaller, M. et al. 2015, MNRAS, 454, 2277

Schaye, J. 2001, ApJ, 562, L95

Schaye, J. 2004, ApJ, 609, 667

Schaye, J. et al. 2015, MNRAS, 446, 521

Schaye, J. \& Dalla Vecchia, C. 2008, MNRAS, 383, 1210

Schaye, J. et al. 2010, MNRAS, 402, 1536

Schaye, J. et al. 2000, MNRAS, 318, 817

Schreiber, C. et al. 2015, A\&A, 575, A74

Sersic, J. L. 1968, Atlas de Galaxias Australes

Shen, S. et al. 2003, MNRAS, 343, 978

Sijacki, D. et al. 2008, MNRAS, 387, 1403

Silk, J. \& Mamon, G. A. 2012, Research in Astronomy and Astrophysics, 12, 917

Sillero, E. et al. 2017, MNRAS, 472, 4404

Somerville, R. S. \& Davé, R. 2015, ARA\&A, 53, 51

Sparke, L. S. \& Gallagher, John S., I. 2007, Galaxies in the Universe: An Introduction

Springel, V. 2005, MNRAS, 364, 1105

Springel, V., Di Matteo, T. \& Hernquist, L. 2005, MNRAS, 361, 776

Springel, V., Frenk, C. S. \& White, S. D. M. 2006, Nature, 440, 1137

Springel, V. \& Hernquist, L. 2002, MNRAS, 333, 649

Springel, V. \& Hernquist, L. 2003, MNRAS, 339, 289

Springel, V. et al. 2018, MNRAS, 475, 676

Springel, V. et al. 2001, MNRAS, 328, 726

Stevens, A. R. H., Croton, D. J. \& Mutch, S. J. 2016, MNRAS, 461, 859 
Stott, J. P. et al. 2014, MNRAS, 443, 2695

Tecce, T. E. et al. 2010, MNRAS, 408, 2008

Thielemann, F.-K. et al. 2003, in From Twilight to Highlight: The Physics of Supernovae, ed. W. Hillebrandt \& B. Leibundgut, 331

Tinker, J. et al. 2008, ApJ, 688, 709

Tissera, P. B. et al. 2016, MNRAS, 456, 2982

Tissera, P. B. et al. 2019, MNRAS, 482, 2208

Trayford, J. W. et al. 2017, MNRAS, 470, 771

Trayford, J. W. et al. 2020, MNRAS, 491, 3937

Trayford, J. W. \& Schaye, J. 2019, MNRAS, 485, 5715

Trayford, J. W. et al. 2016, MNRAS, 460, 3925

Trayford, J. W. et al. 2015, MNRAS, 452, 2879

Tremonti, C. A. et al. 2004, ApJ, 613, 898

Troncoso, P. et al. 2014, A\&A, 563, A58

van de Sande, J. et al. 2019, MNRAS, 484, 869

van de Voort, F. 2017, in Astrophysics and Space Science Library, Vol. 430, Gas Accretion onto Galaxies, ed. A. Fox \& R. Davé, 301

van de Voort, F. et al. 2011, MNRAS, 414, 2458

Veilleux, S., Cecil, G. \& Bland-Hawthorn, J. 2005, ARA\&A, 43, 769

Vogelsberger, M. et al. 2013, MNRAS, 436, 3031

Vogelsberger, M. et al. 2014, Nature, 509, 177

Vogelsberger, M. et al. 2018, MNRAS, 474, 2073

Vogelsberger, M. et al. 2020, Nature Reviews Physics, 2, 42

von Steiger, R. \& Zurbuchen, T. H. 2016, ApJ, 816, 13

Wetzel, A. R. et al. 2016, ApJ, 827, L23

White, S. D. M., Efstathiou, G. \& Frenk, C. S. 1993, MNRAS, 262, 1023

White, S. D. M. \& Rees, M. J. 1978, MNRAS, 183, 341

Wiersma, R. P. C., Schaye, J. \& Smith, B. D. 2009a, MNRAS, 393, 99

Wiersma, R. P. C. et al. 2009b, MNRAS, 399, 574

Winther, H. A. et al. 2015, MNRAS, 454, 4208

Wright, R. J. et al. 2019, MNRAS, 487, 3740 
Wright, R. J. et al. 2020, MNRAS, 498, 1668

Wright, T. 1750, An original theory or new hypothesis of the Universe: Founded upon the laws of nature, and solving by mathematical principles the general phaenomena of the visible creation; and particularly the Via Lactea

Wu, Y.-Z. et al. 2016, MNRAS, 457, 2929

Wuyts, E. et al. 2016, ApJ, 827, 74

Xie, L. et al. 2020, MNRAS, 498, 4327

Xie, L. et al. 2017, MNRAS, 469, 968

Yates, R. M., Thomas, P. A. \& Henriques, B. M. B. 2017, MNRAS, 464, 3169

Yuan, F. \& Narayan, R. 2014, ARA\&A, 52, 529

Zahid, H. J. et al. 2012, ApJ, 750, 120

Zahid, H. J. et al. 2014a, ApJ, 791, 130

Zahid, H. J. et al. 2013, ApJ, 771, L19

Zahid, H. J. et al. 2014b, ApJ, 792, 75

Zinchenko, I. A. et al. 2019, A\&A, 623, A7 


\section{Apéndice A}

\section{Masas de oxígeno en las distintas componentes bariónicas de SAG}

En el Capítulo 3 presentamos los resultados de nuestra investigación sobre el impacto del feedback de SN en la evolución de la MZR. Dado que la MZR involucra la abundancia del oxígeno en la fase de gas frío, analizamos el contenido de masa de este elemento químico en las diferentes componentes bariónicas modeladas por SAG. La Figura A.1 muestra la masa de oxígeno como función de la masa estelar a redshift $z=0,2.2$ y 3,5 en los paneles superior, intermedio e inferior, respectivamente, para galaxias generadas por el modelo $\mathrm{SAG}_{\beta 1.9}$. Mostramos las contribuciones de la masa de oxígeno de las distintas componentes bariónicas, a saber, la fase de gas caliente (línea sólida), la fase de gas frío (línea sólida gruesa), la masa de oxígeno encerrada en las estrellas (línea sólida fina) y la masa total de oxígeno producida por las estrellas en las galaxias (línea a trazos). Esta última es la suma en el tiempo del oxígeno producido por las estrellas en la galaxia, $\Delta M_{\mathrm{ej}}^{\mathrm{O}}+\Delta M_{\mathrm{ej}(\mathrm{Ia})}^{\mathrm{O}}$ (involucra Ecuaciones 2.18 y 2.19). A pesar de que la componente de gas eyectado (Ecuación 2.21) contenga algo de oxígeno, no se muestra en la Figura debido a que típicamente es varios órdenes de magnitud menor que en las otras componentes bariónicas (los valores medios de su masa de oxígeno no superan la cantidad de $\approx 10^{4} \mathrm{M}_{\odot}$ en ningún redshift). Esto responde a que la componente de masa eyectada generalmente es una pequeña contribución a la fracción bariónica, excepto en halos de baja masa. El anterior es un comportamiento general de los modelos semi-analíticos (Lagos et al., 2018b).

La mayor parte de la masa de oxígeno producida por las estrellas está ubicada en la fase caliente del gas, independientemente de la masa estelar y redshift en la que la galaxia se encuentre. Sin embargo, cuando el tiempo pasa, la diferencia entre la masa de oxígeno en el gas caliente y frío se vuelve más pequeña. Además, para galaxias con $M_{\star} \leq 10^{10-10.5} \mathrm{M}_{\odot}$, la cantidad de oxígeno encerrada en las estrellas es menor que la que hay en la fase fría del gas, lo que es opuesto a lo que obtenemos para galaxias de $M_{\star} \geq 10^{10-10.5} \mathrm{M}_{\odot}$. Esta inversión tiene lugar en galaxias masivas a medida que la masa de la reserva de gas frío se vuelve más chica que su masa estelar (ver la relación entre la fracción de gas frío y la masa estelar a $z=0$ en la Figura 2.3), que naturalmente lleva a que la cantidad de oxígeno contenida en el gas frío sea menor que la que está encerrada en estrellas. Debido a que el oxígeno en el gas caliente domina la cantidad total de oxígeno en el sistema halo-galaxia, es esperable que la evolución exacta de la metalicidad del gas caliente tenga un fuerte impacto en la evolución de la metalicidad de la galaxia. 

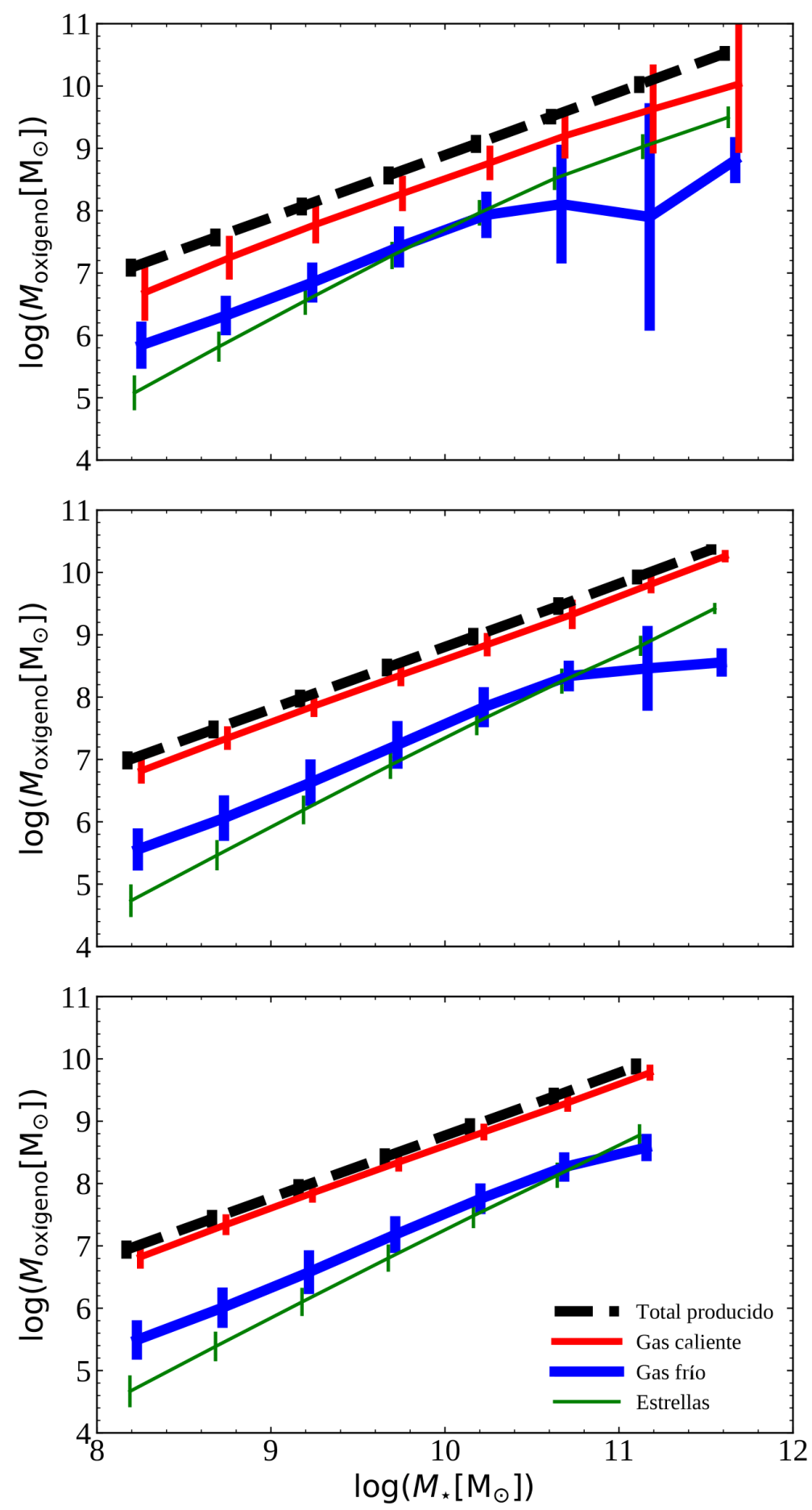

Figura A.1: Masa de oxígeno de galaxias que forman estrellas para el modelo $\mathrm{SAG}_{\beta 1.9}$ en función de la masa estelar a $z=0$ (panel superior), $z=2.2$ (panel intermedio), y $z=3.5$ (panel inferior). Las líneas negra a trazos, roja sólida, azul sólida gruesa y verde sólida fina representan, respectivamente, los valores medios del contenido total de la masa de oxígeno producida por la galaxia durante la nucleosíntesis, el oxígeno de la masa de gas caliente, el oxígeno contenido en el gas frío, y la cantidad de oxígeno encerrado en estrellas. La dispersión de $1 \sigma$ es representada por las barras de error. En todos los redshifts, la mayoría de la masa de oxígeno se encuentra en la fase de gas caliente. 


\section{Apéndice B}

\section{Características de la relación $Z_{\mathrm{hot}}-M_{\star}$ a $z=0$ en SAG}

\section{B.1. Relación entre $M_{\text {halo }}$ y la dispersión en la relación $Z_{\text {hot }}-M_{\star}$}

En el Capítulo 3, describimos el análisis del enriquecimiento químico de la fase caliente del gas de las galaxias en función de su masa estelar. Esta relación se caracteriza por una tendencia invertida a altas masas estelares para $z \lesssim 2$ y con una gran dispersión comparada con lo esperado para el ICM, como se ve en la Figura 3.8. Con el fin de entender estas características de la relación $Z_{\mathrm{hot}}-M_{\star}$, estimamos los valores promedio de $Z_{\text {hot }}$ de las galaxias $S F$ en $\mathrm{SAG}_{\beta 1.9}$, considerando intervalos de masa de DM del (sub)halo, $M_{\text {halo }}$, en los que las galaxias yacen. $M_{\text {halo }}$ está dada por la masa de cada (sub)estructura contenida dentro de $r_{200}$, que es el radio donde la densidad de materia es 200 veces la densidad crítica del Universo, es decir, $M_{\text {halo }}=M_{\mathrm{DM}}\left(r_{200}\right)$. El panel superior izquierdo de la Figura 3.8 muestra el resultado de tres rangos de masa de halo, a saber, $M_{\text {halo }} / \mathrm{M}_{\odot} \in\left[10^{12}, 10^{13}\right],\left[10^{13}, 10^{14}\right]$ y $\left[10^{14}, 10^{16}\right]$. Como puede verse, la metalicidad del gas caliente de galaxias que habitan halos de baja masa es mayor para galaxias de alta masa estelar, produciendo una gran dispersión. En cambio, galaxias en los intervalos de masa de halo más bajas muestran un incremento de los valores promedio de $Z_{\text {hot }}$ con la masa estelar; aquellas galaxias con $M_{\star} \gtrsim 5 \times 10^{10} \mathrm{M}_{\odot}$ residiendo en los dos intervalos de masa de halo más masivos son responsables del decaimiento de $Z_{\text {hot }}$. Entonces, tanto las dispersiones como la inversión de la relación están fuertemente correlacionados con la masa del halo.

El gas caliente de las galaxias centrales residiendo en los dos intervalos de masa de halo más grandes representan el ICM. Se encuentra que la población de galaxias con $M_{\star} \gtrsim 10^{11} \mathrm{M}_{\odot}$ es dominada por centrales. En efecto, para los halos dentro de los rangos de masa $M_{\text {halo }} / \mathrm{M}_{\odot} \in\left[10^{12}, 10^{13}\right]$, $\left[10^{13}, 10^{14}\right]$ y $\left[10^{14}, 10^{16}\right]$, el porcentaje correspondiente de galaxias centrales con respecto al número total de galaxias (centrales más satélites) seleccionadas de acuerdo a este corte de masa estelar es $\approx 88 \%, 94 \%$ y $97 \%$. Así, los valores promedio de $Z_{\text {hot }}$ para diferentes intervalos de masa de halo pueden ser comparados con la metalicidad observada del gas caliente que rodea los grupos y cúmulos de galaxias presentados por Yates et al. (2017, representados por estrellas en el panel superior izquierdo de la Figura 3.8), aún cuando la muestra completa de galaxias modeladas incluye también galaxias satélites. Estos autores mostraron la relación entre la abundancia del hierro (pesada por masa) dentro de $r_{500}, \bar{Z}_{\mathrm{Fe}, 500}$, y la temperatura del gas caliente $T_{500}=T\left(r_{500}\right)$ del ICM para una muestra homogeneizada de grupos y cúmulos compilada de la literatura, donde $r_{500}$ es el análogo a $r_{200}$ pero la densidad de materia es 500 veces la densidad crítica. La conversión de $\bar{Z}_{\mathrm{Fe}, 500}-T_{500}$ a $Z_{\mathrm{hot}}-M_{\star}$ puede verse a continuación en la Sección B.2. Considerando las galaxias dentro de halos de $M_{\text {halo }}>10^{13} \mathrm{M}_{\odot}$, que es el rango considerado por Yates et al. (2017), podemos ver que la metalicidad del gas caliente predicha por el modelo $\mathrm{SAG}_{\beta 1.9}$ está en buen acuerdo con los datos observacionales. Sin embargo, las galaxias modeladas con $M_{\star} \gtrsim 10^{11} \mathrm{M}_{\odot}$ dentro de halos de $M_{\text {halo }} / \mathrm{M}_{\odot} \in\left[10^{14}, 10^{16}\right]$ muestran 
una tendencia opuesta, con $Z_{\text {hot }}$ decreciendo a medida que la masa estelar crece. Entendemos que este comportamiento es debido a que el modelo SAG produce una alta tasa de fusiones de manera artificial para galaxias a $z<2$ (discutido en detalle en C18). Debido a esto, los halos calientes de galaxias centrales adquieren demasiado gas con metalicidades bajas de las galaxias de baja masa con las que se están fusionando, resultando en una dilución de la metalicidad. De manera similar, Yates et al. (2017) señala que un proceso importante para la dilución de la metalicidad del gas caliente es la caída de satélites en sistemas masivos, lo que favorece la acreción de gas prístino o de baja metalicidad.

\section{B.2. Conversión de $\bar{Z}_{\mathrm{Fe}, 500}-T_{500}$ a $Z_{\mathrm{hot}}-M_{\star}$}

Los valores promedio de la metalicidad de gas caliente, $Z_{\text {hot }}$, de las galaxias de $\mathrm{SAG}_{\beta 1.9}$ son presentados como función de la masa estelar en la Figura 3.8 (panel superior izquierdo). Los resultados modelados son comparados con las abundancias de hierro observadas del gas caliente, $\bar{Z}_{\mathrm{Fe}, 500}{ }^{1}$, que rodea los grupos y cúmulos presentados por Yates et al. (2017). Dado que sus abundancias de hierro están dadas en función de la temperatura del gas caliente, $T_{500}$, debemos realizar dos conversiones basadas en los resultados del modelo.

Por un lado, para convertir los valores de $\bar{Z}_{\mathrm{Fe}, 500}$ de las observaciones a $Z_{\mathrm{hot}}$, aplicamos la correlación entre la metalicidad del gas caliente y la abundancia del hierro en el gas caliente que observamos internamente en $\mathrm{SAG}_{\beta 1.9}$. Esto es $Z_{\mathrm{hot}}=1.55 \log \left(Z_{\mathrm{Fe}, \mathrm{SAG}}\right)+1.92$, lo que ya tiene en cuenta los diferentes valores solares utilizados para normalizar cada una de estas cantidades. Recordamos que, en el modelo, $Z_{\text {hot }}$ ha sido normalizada con el valor solar de $Z_{\odot}=0.0196$ (von Steiger \& Zurbuchen, 2016). La abundancia de hierro $Z_{\mathrm{Fe}, \mathrm{SAG}}$ es referida al mismo valor solar adoptado por Yates et al. (2017). Luego, usamos los valores observados de $\bar{Z}_{\mathrm{Fe}, 500}$ en lugar de $\mathrm{Z}_{\mathrm{Fe}, \mathrm{SAG}}$ como entrada en esta relación para obtener la correspondiente $Z_{\text {hot }}$.

Por otro lado, obtenemos la relación entre la masa estelar y la temperatura del gas a $r_{500}$ del modelo. Este radio es obtenido de $r_{200}$ (dadas las salidas de la simulación de DM subyacente), adoptando un perfil de densidad isotermo. La temperatura del gas caliente de las galaxias modeladas, $T_{500, \mathrm{SAG}}$, es estimada siguiendo el procedimiento descrito en Yates et al. (2017, ver su sección 2.3), en donde se necesita la temperatura a $r_{200}, T_{200}$. Esta propiedad es calculada mediante la aplicación de la conversión entre la velocidad virial y temperatura de Springel et al. (2001), $T_{200}=35.9\left(V_{\text {vir }} / \mathrm{km} \mathrm{s}^{-1}\right)^{2} \mathrm{~K}$, la cual juega un rol clave en la clasificación de ambientes de galaxias (Yates et al., 2017). Los grupos son definidos como aquellos halos con temperatura $k_{\mathrm{B}} T_{200}<2.5 \mathrm{kev}\left(k_{\mathrm{B}}\right.$ es la constante de Boltzmann), mientras que los cúmulos son caracterizados por $k_{\mathrm{B}} T_{200}>2.5 \mathrm{kev}$. Adoptando diferentes perfiles de temperatura para grupos y cúmulos (dadas las ecuaciones 6 y 8 de Yates et al. 2017), es-

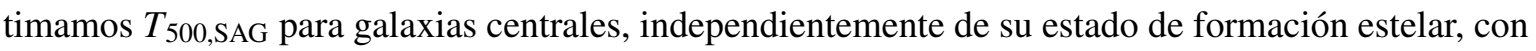
la condición de que su $M_{\text {halo }} \geq 10^{13} \mathrm{M}_{\odot}$, para realizar una comparación justa con las observaciones. Entonces, la masa estelar de las galaxias centrales dentro de halos con temperatura de gas observada, $T_{500}$, es obtenida a través de la conexión entre $T_{500, \text { SAG }} \mathrm{y} M_{\star}$ encontrada a partir del modelo.

\footnotetext{
${ }^{1}$ Aquí usamos que $\log \left(\mathrm{Z}_{\mathrm{Fe}}\right) \equiv\left[\frac{\mathrm{Fe}}{\mathrm{H}}\right]=\log \left(\frac{N_{\mathrm{Fe}}}{N_{\mathrm{H}}}\right)-\log \left(\frac{N_{\mathrm{Fe}, \odot}}{N_{\mathrm{H}, \odot}}\right)$, siendo la abundancia solar del hierro $\left(\frac{N_{\mathrm{Fe}, \odot}}{N_{\mathrm{H}, \odot}}\right)=3.16 \times 10^{-5}$ (Grevesse \& Sauval, 1998).
} 


\section{Apéndice C}

\section{Convergencia de la relación $\dot{M}_{\text {accr }}-$ RMP en EAGLE}

Para el análisis realizado en el Capítulo 4, consideramos galaxias SF centrales con al menos 10 partículas de gas SF que han sido acretadas (que estaban fuera de las galaxias hace $\approx 100 \mathrm{Myr}$ ). Este mínimo de partículas se traduce en un valor de $\dot{M}_{\mathrm{accr}} \approx 9 \times 10^{-2} \mathrm{M}_{\odot} \mathrm{yr}^{-1}$. Aquí, evaluamos la robustez de nuestros resultados respecto del número mínimo de partículas de gas acretado permitidas.

Mostramos en la Figura C.1 la relación entre los residuos de la pendiente del RMP, $\Delta \alpha$, y los residuos de la $\dot{M}_{\text {accr }}, \Delta \dot{M}_{\text {accr }}$, para cuatro cortes en el mínimo de número de partículas de gas $\mathrm{SF}$, desde al menos 1 partícula a 100 partículas, como muestra la leyenda. La definición de residuos se encuentra en la Sección 4.5.2. Por claridad, sólo mostramos la dispersión de $1 \sigma$ para nuestro corte original de 10 partículas para ambos modelos (la región sombreada magenta muestra la simulación Recal-L25N752, mientras que la cian representa la simulación Ref-L100N1504).

Podemos ver que, para todo redshift analizado, prácticamente no hay diferencia entre los cuatro cortes de mínimo número de partículas de gas. Es interesante analizar los casos donde las galaxias tienen menos de 10 partículas de gas SF acretadas. Para la simulación Ref-L100N1504, 17 galaxias a $z=0(\approx 10 \%$ de la muestra a ese redshift $)$ tienen menos de 10 partículas y muestran las mismas tendencias que las reportadas en el Capítulo 4. A $z=1$, sólo una galaxia de esta simulación tiene menos de 10 partículas de gas acretadas (lo que corresponde a $\dot{M}_{\text {accr }} \approx 10^{-2.5} \mathrm{M}_{\odot} \mathrm{yr}^{-1}$ y $\alpha \approx 0.015$ ). Por el contrario, la simulación Recal-L25N752 no tiene ninguna galaxia SF central con menos de 10 partículas de gas SF siendo acretadas (al menos hasta $z \leq 1$ ). Por lo tanto, concluimos que nuestros resultados son robustos con el mínimo número elegido de 10 partículas de gas $\mathrm{SF}$ acretadas. 

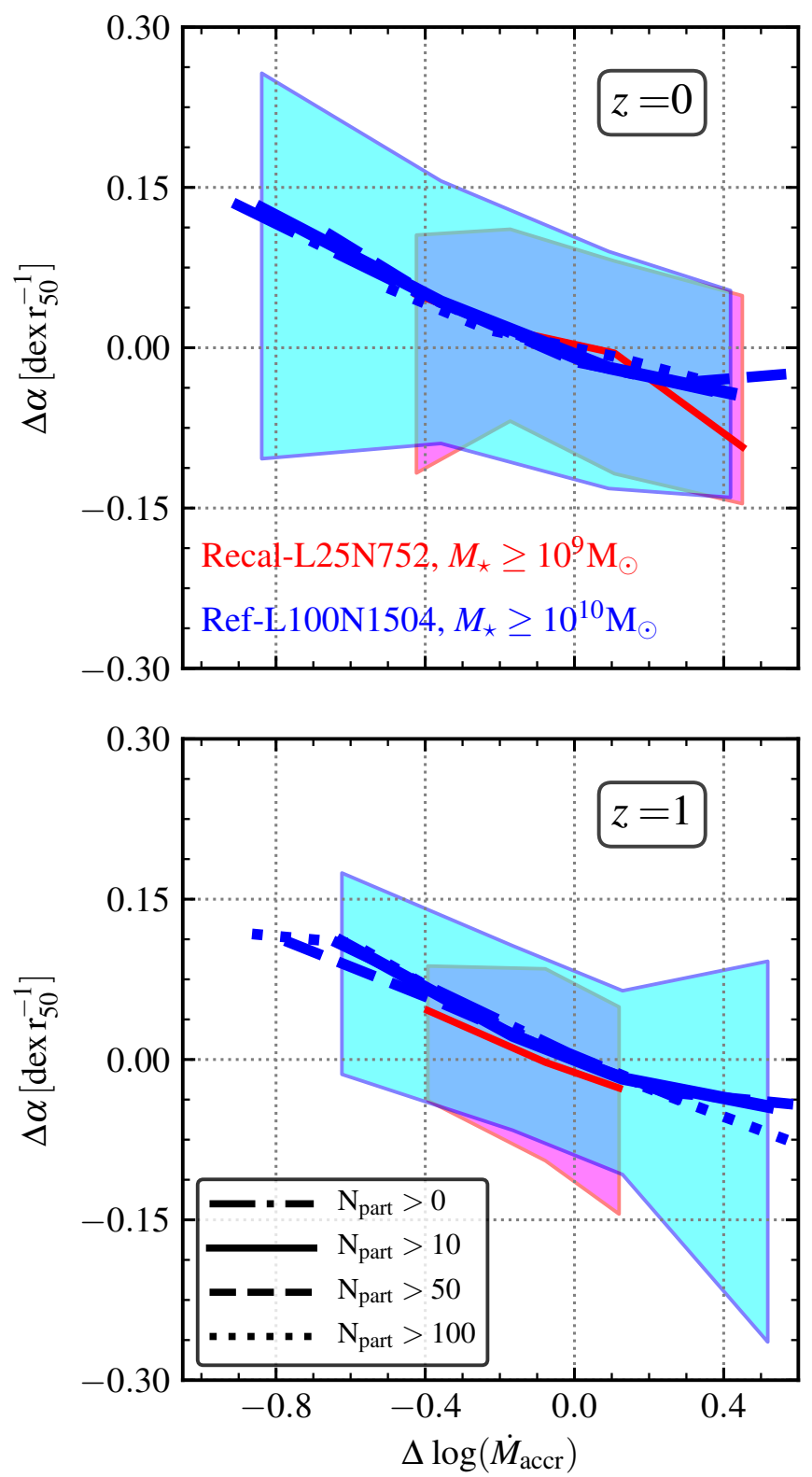

Figura C.1: Residuos de la relación $\alpha-M_{\star}$ como función de los residuos de la relación $\dot{M}_{\text {accr }}-M_{\star}$, con $\alpha$ siendo la pendiente interna del RMP $\left(r / r_{50} \leq 1\right)$. Los paneles superior e inferior muestran galaxias a $z=0$ y $z=$ 1, respectivamente. Los colores rojo y azul representan las simulaciones Recal-L25N752 y Ref-L100N1504, respectivamente. Esta Figura es similar a la Figura 4.4, pero ahora se comparan los diferentes números mínimos de partículas de gas en el gas acretado. Líneas sólida, a trazos y punteada muestran la $\dot{M}_{\text {accr }}$ de galaxias con $\geq 10, \geq 50 \mathrm{y} \geq 100$ partículas de gas acretado, respectivamente. Las barras de error muestran la dispersión de $1 \sigma$ sólo para el caso de $\geq 10$ partículas de gas por claridad. 


\section{Apéndice D}

\section{Comparación de la pendiente de los RMP con trabajos anteriores}

A lo largo del Capítulo 4, trabajamos con unidades galactocéntricas en función del radio pesado por el radio mitad de masa estelar, es decir de $r / r_{50}$. Esto nos permite trabajar con las galaxias independientemente de su tamaño, además que nos proporciona una manera de separar a las galaxias en regiones internas y externas. Sin embargo, en la bibliografía se pueden encontrar diversos cortes en radio para calcular la pendiente del RMP, es decir $\alpha$. Así, el corte podría darse por el radio en el que la galaxia alcanza el $25 \%$ de su brillo (o masa), podría calcularse el perfil hasta alcanzar un múltiplo de este radio (por ejemplo, $2.5 \times \mathrm{R}_{25}$ ), podría calcularse la pendiente sin realizar corte alguno en radio, o hasta podría no pesarse el radio. En este último caso, la pendiente $\alpha$ tiene unidades de dex kpc ${ }^{-1}$.

Nos preguntamos, entonces, si los valores de la pendiente hallados en el Capítulo 4 son consistente con trabajos anteriores. Para ello, tomamos resultados de trabajos que no pesen el radio al calcular el perfil, y los comparamos con nuestros resultados de la pendiente interda (o sea, la pendiente que se obtiene al calcular el RMP hasta $r=1 \times r_{50}$ ) en las unidades correspondientes. Dado que la mayor cantidad de material se encontrará en la región interna de la galaxia, esto nos da una buena aproximación de si las pendientes halladas en esta Tesis tienen valores acordes a lo hallado previamente, a pesar de la diferencia en el cálculo para obtenerlas.

En la Figura D.1 mostramos la relación entre la pendiente $\alpha$ en unidades de dex $\mathrm{kpc}^{-1}$ como función de la masa estelar a $z=0$. Aunque una comparación con las observaciones no es directa (debido a las diferencias en el cálculo de la pendiente), mostramos en este Apéndice nuestros resultados comparados con las observaciones de Belfiore et al. (2017) (círculos verde llenos) y Ho et al. (2015) (diamantes negros llenos), y los resultados de simulaciones previos de Tissera et al. (2016) (diamantes rosas), Ma et al. (2017) (círculos violetas) y Tissera et al. (2019) (línea punteada verde). Vemos que las pendientes halladas por nuestro método están en buen acuerdo con los resultados previos (sean observacionales o teóricos), aunque para una mejor comparación es necesario un juego de observaciones que adopte el radio $r_{50}$. 


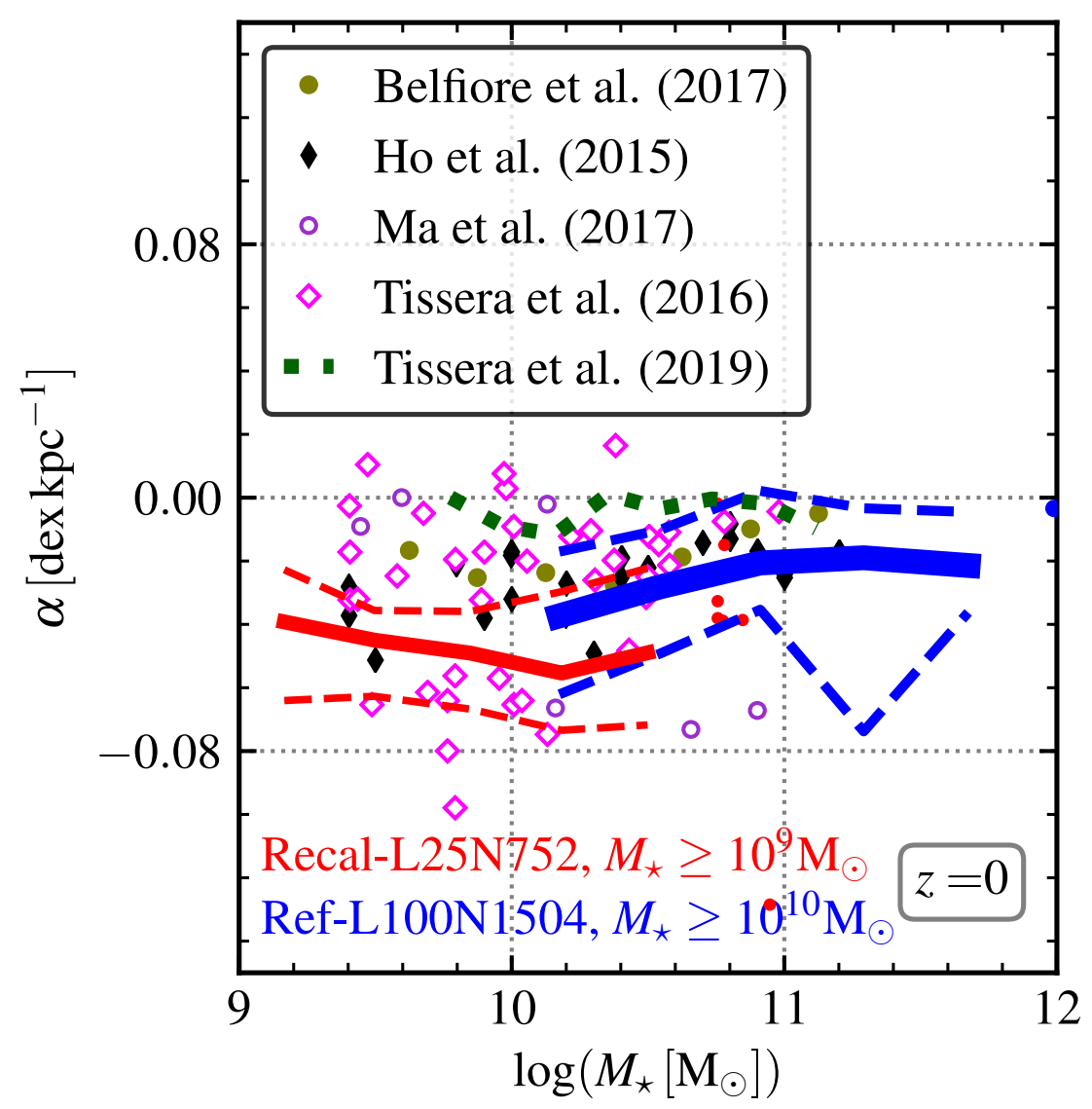

Figura D.1: Relación entre la pendiente interna $\alpha$ en unidades de dex $\mathrm{kpc}^{-1}$, como función de la masa estelar a $z=0$ para las simulaciones Recal-L25N752 (rojo) y Ref-L100N1504 (azul). A pesar de que la comparación de nuestros resultados con las observaciones no es directa debido a la diferencia en el cálculo de la pendiente $\alpha$, esta Figura muestra algunos resultados observacionales y teóricos, a fin de mostrar la similitud en resultados. Los valores medianos están representado por las líneas sólidas, mientras que los percentiles 16-84 se muestran con líneas a trozos. Los cículos verdes llenos y los diamantes negros llenos denotan las observaciones de Belfiore et al. (2017) y Ho et al. (2015), respectivamente. Por otro lado, los resultados observacionales de Ma et al. (2017), Tissera et al. (2016) y Tissera et al. (2019) se muestran en círculos violetas, diamantes rosas y línea a trozos verde, respectivamente. 


\section{Apéndice E}

\section{Tasa de acreción de gas como función de la masa estelar en EAGLE}

En el Capítulo 4 mostramos la MZR en intervalos de $\dot{M}_{\text {accr }}$, en donde ya se advierten cambios en la metalicidad de las galaxias a masa estelar fija debido a la $\dot{M}_{\text {accr }}$. Para poder entender en más detalle cómo la $\dot{M}_{\text {accr }}$ afecta la evolución química de las galaxias, es relevante analizar cómo la $\dot{M}_{\text {accr }}$ depende de la masa estelar, $M_{\star}$.

La Figura E.1 muestra la relación $\dot{M}_{\text {accr }}-M_{\star}$ a redshift $z=0$ (panel superior) y $z=1$ (panel inferior). Las líneas sólidas muestran los valores medianos, mientras que las líneas a trazos representan los percentiles 16 - 84. La línea negra punteada a trazos en una función lineal con pendiente $\equiv 1$ para usar de referencia.

Como es esperado, la relación $\dot{M}_{\text {accr }}-M_{\star}$ tiene una pendiente positiva, es decir, que galaxias con $M_{\star}$ creciente también muestran una creciente $\dot{M}_{\text {accr }}$, y, a masa estelar fija, $\dot{M}_{\text {accr }}$ decrece con el tiempo. Esta relación es bastante ajustada (alrededor de $\approx 0.5$ dex de dispersión, cuantificada por los percentiles 16-84). Puede verse un pequeño desplazamiento entre las dos simulaciones para galaxias con $M_{\star} \approx 10^{10} \mathrm{M}_{\odot}$, aunque no tan pronunciado como en la Figura 4.3. Este desplazamiento no es inesperado dado los diferentes ambientes en que residen las galaxias de ambas simulaciones (Furlong et al., 2015). A pesar de esto, vemos que una vez que prescindimos de la dependencia con la masa estelar, emerge una relación más clara entre la $\dot{M}_{\text {accr }}$ y la pendiente del RMP. 

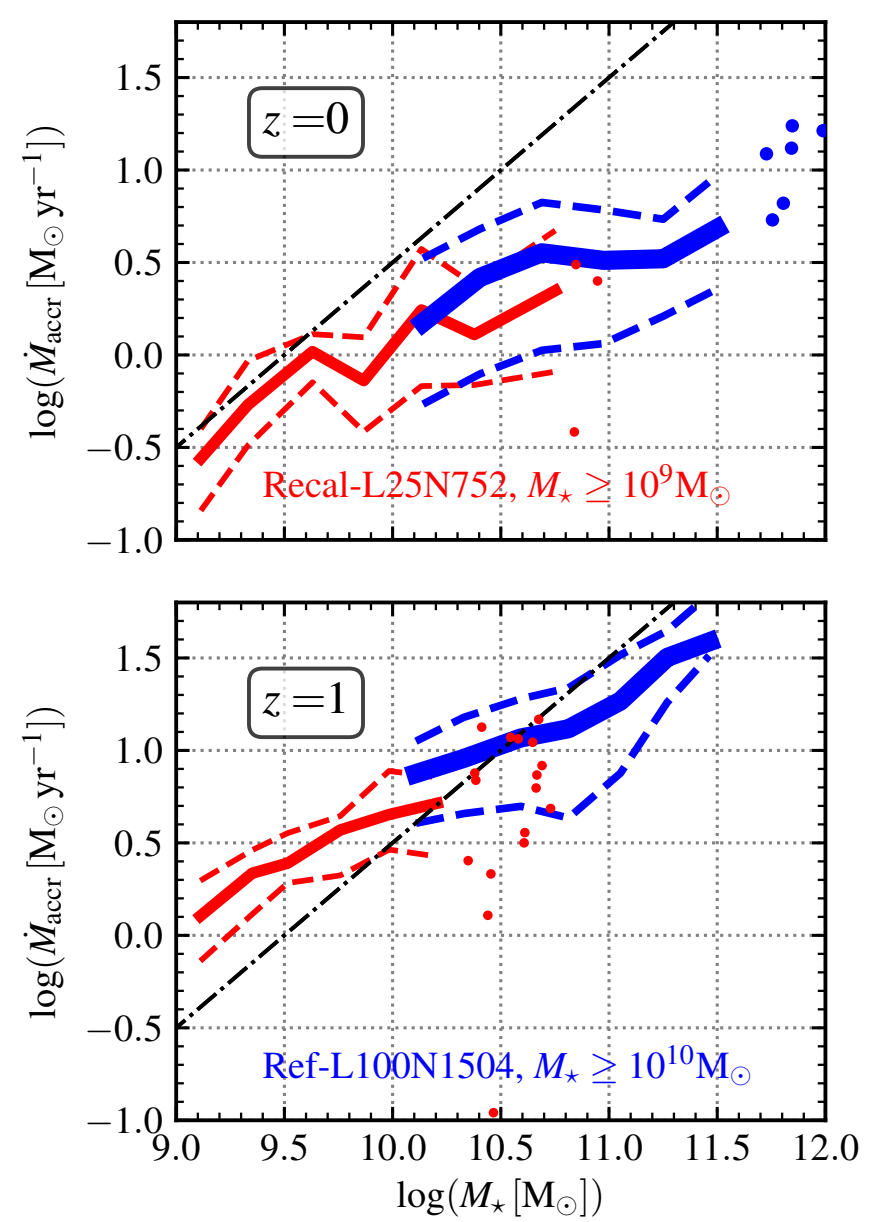

Figura E.1: $\dot{M}_{\text {accr }}$ como función de la masa estelar a redshift $z=0$ (panel superior) y $z=1$ (panel inferior). Las simulaciones Recal-L25N752 y Ref-L100N1504 son mostradas en rojo y azul, respectivamente. Las líneas sólidas muestran los valores medios de la relación, mientras que las líneas a trazos representan los percentiles 16 - 84. Se muestran las galaxias individuales en los intervalos con $<10$ objetos (símbolos). La línea punteada a trazos muestra la función $\log _{10}\left(\dot{M}_{\text {accr }}\right)=\log _{10}\left(M_{\star}\right)+9.5$, a modo de referencia. 


\section{Apéndice F}

\section{Relación entre la fracción de hidrógeno molecular y la $\dot{M}_{\text {accr }}$ en EAGLE}

En el Capítulo 4 mostramos los cambios de la pendiente de los RMPs, $\alpha$, como función de la fracción de gas de las galaxias, en donde nuestra definición de fracción de gas involucra tanto el gas molecular $\mathrm{H}_{2}$ como el gas atómimco $\mathrm{HI}$. Dado que observacionalmente es más sencillo estimar el $\mathrm{H}_{2}$ que el $\mathrm{HI}$ a $z \gtrsim 0.5$, testeamos si nuestras conclusiones de la Sección 4.5.4 podrían cambiar si consideramos solamente el primero.

La Figura F.1 muestra los cambios de la pendiente $\alpha$ y sus residuos en función de la fracción de gas, cuya definición varía con respecto a la utilizada en la la Figura 4.7. Ahora, consideramos sólo la contribución del $\mathrm{H}_{2} \mathrm{y}$, así, tenemos que la fracción de gas resulta definida como $f_{\mathrm{gas}}=M_{\mathrm{H}_{2}} /\left(M_{\star}+\mathrm{H}_{2}\right)$. A primera vista, una de las diferencias que notamos es que los valores de $f_{\text {gas }}$ caen considerablemente comparados con nuestra definición previa (alcanzan valores de hasta 0.15 y 0.25 a $z=0$ y $z=1$, respectivamente, en comparación con valores de 0.7 para ambos redshifts que obtuvimos mediante el análisis detallado en el Capítulo 4). Esto se debe al hecho de que $M_{\mathrm{HI}}$ es más abundante que la componente molecular. Otro hecho interesante para remarcar es que los valores máximos de $f_{\text {gas }}$ incrementan con el aumento de redshift, mostrando la razón por la cual es más sencillo estimar observacionalmente $M_{\mathrm{H}_{2}}$ a redshift $z \gtrsim 0.5$. Debido a que las tendencias encontradas tanto en la Sección 4.5.4 como aquí son similares (independientemente de los valores que alcanza $f_{\text {gas }}$ ), concluimos que tanto si la definición de la fracción de gas involucra $\mathrm{H}_{2} \mathrm{O} \mathrm{HI}$ (no se muestra), los resultados encontrados en el Capítulo 4.5.4 permanencen iguales. 

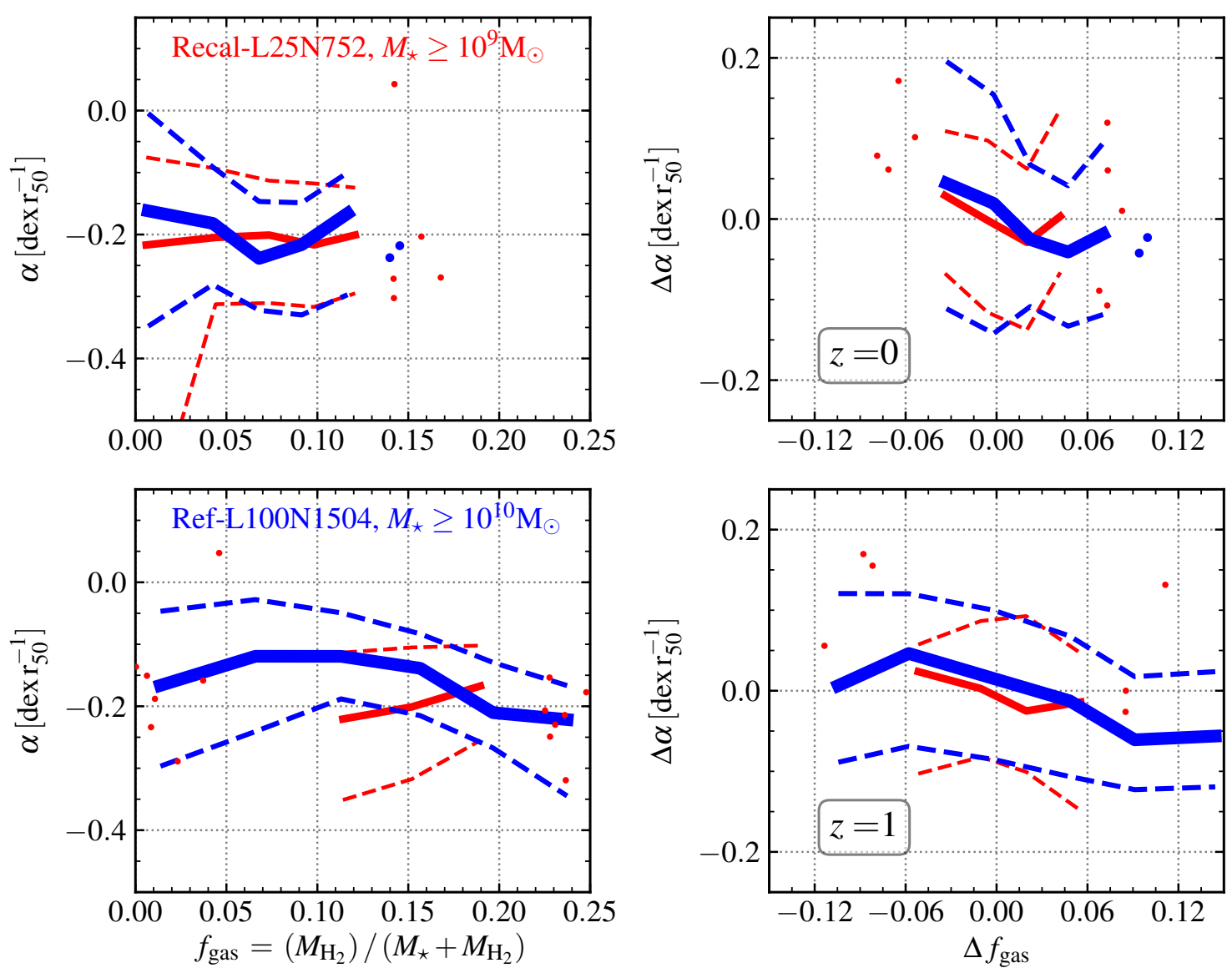

Figura F.1: Izquierda: Las líneas sólidas muestran los valores medios de la pendiente interna del RMP $\left(r / r_{50} \leq\right.$ 1) como función de la fracción de gas molecular (definida como $f_{\text {gas }}=\mathrm{H}_{2} /\left(M_{\star}+\mathrm{H}_{2}\right)$ ) a redshift $z=0$ (panel superior) y a $z=1$ (panel inferior), para las simulaciones Recal-L25N752 (rojo) y Ref-L100N1504 (azul). Derecha: Las líneas sólidas muestran los valores medios de los residuos de la relación entre la pendiente interna del RMP y la $M_{\star}$, como función de los residuos de la fracción de gas molecular $\left(f_{\text {gas }}=\mathrm{H}_{2} /\left(M_{\star}+\mathrm{H}_{2}\right)\right)$ y la $M_{\star}$. Todos los paneles: los percentiles $16-84$ son denotados con líneas a trazos. Mostramos galaxias individuales donde los intervalos tienen $<10$ objetos (círculos rojos). 


\section{Apéndice G}

\section{Influencia del tamaño de spaxels en la estimación de propiedades locales de las galaxias}

En el Capítulo 5 presentamos resultados sobre el estudio de la metalicidad y otras propiedades locales de galaxias considerando regiones de $1 \mathrm{kpc} \times 1 \mathrm{kpc}$, las cuales denominamos spaxels. La determinación del tamaño de estos spaxels se basa en dos condiciones. Por un lado, observacionalmente se elige una región con una dimensión de $\sim 2.5$ segundos de arco para poder definir si el valor de una determinada propiedad es alto o bajo; a $z=0$, esta medida angular equivale a un tamaño similar al que tomamos para nuestros spaxels. Por otro lado, el límite de resolución de las simulaciones utilizadas no nos permite tomar spaxels más pequeños sin perder confianza en los resultados, al menos para la simulación Ref-L100N1504. El hecho de que los resultados que obtuvimos se modifiquen dependiendo del tamaño de los spaxels puede llegar a relacionarse con el grado de confiabilidad de las simulaciones hidrodinámicas a la hora de representar propiedades locales. En ese caso, las propiedades globales podrían estar relativamente bien entendidas, aunque no así la física de la que derivan esas propiedades. En este sentido, la evaluación de los cambios y similitudes de los análisis planteados al modificar el tamaño de los spaxels resulta imperativa. Dado que la simulación Recal-L25N752 tiene una longitud máxima de suavizado (softening) de $0.35 \mathrm{kpc}$, se podrían tomar spaxels de menor tamaño sin perder confiabilidad. Realizamos entonces nuevamente los análisis que mostramos en el Capítulo 5, pero utilizando spaxels de dimensión $0.5 \mathrm{kpc} \times 0.5 \mathrm{kpc}$.

Presentamos como ejemplo la Figura G.1. En ella se muestra la relación entre la metalicidad de los spaxels de $0.5 \mathrm{kpc}$ en función de la $f_{\text {accr }}$ para la simulación Recal-L25N752 a $z=0$ (paneles izquierdos) y a $z=1$ (paneles derechos). Los paneles inferiores muestran la PDF de la $f_{\text {accr. }}$. Los valores medianos de la relación están representados por las líneas negras sólidas, mientras que la dispersión de $1 \sigma$ se muestra con las sombras grises. Los símbolos violeta, naranja, verde y rosa denotan la mediana de la relación para spaxels en galaxias de $M_{\star} / \mathrm{M}_{\odot} \in\left[10^{9}, 10^{9.5}\right], M_{\star} / \mathrm{M}_{\odot} \in\left[10^{9.5}, 10^{10}\right]$, $M_{\star} / \mathrm{M}_{\odot} \in\left[10^{10}, 10^{10.5}\right]$ y $M_{\star} / \mathrm{M}_{\odot} \in\left[10^{10.5}, 10^{11}\right]$, respectivamente. Las líneas negras a trazos representan los valores medianos de la simulación Recal-L25N752 utilizando spaxels de $1 \mathrm{kpc}$, a modo de comparación. La relación encontrada para los spaxels de $0.5 \mathrm{kpc}$ resulta extremadamente similar a la que se obtiene con spaxels de $1 \mathrm{kpc}$. Hay una pequeña diferencia entre ambas, tal que la pendiente de la relación obtenida para spaxels de $1 \mathrm{kpc}$ resulta levemente más negativa que la pendiente de la relación obtenida por spaxels de $0.5 \mathrm{kpc}$. Las relaciones que se obtienen para los valores medios de spaxels de $0.5 \mathrm{kpc}$ de lado que se encuentran en galaxias de un particular rango de masa estelar son similares a las obtenidas en la Figura 5.6.

La similitud de los resultados obtenidos al considerar spaxels de $0.5 \mathrm{kpc}$ y de $1 \mathrm{kpc}$ de lado, se encuentra también en el resto de las relaciones analizadas que, por simplicidad, no se muestran en 
este apéndice. Concluimos, entonces, que cambiar los tamaños de los spaxels no afecta los resultados que presentamos en el Capítulo 5.
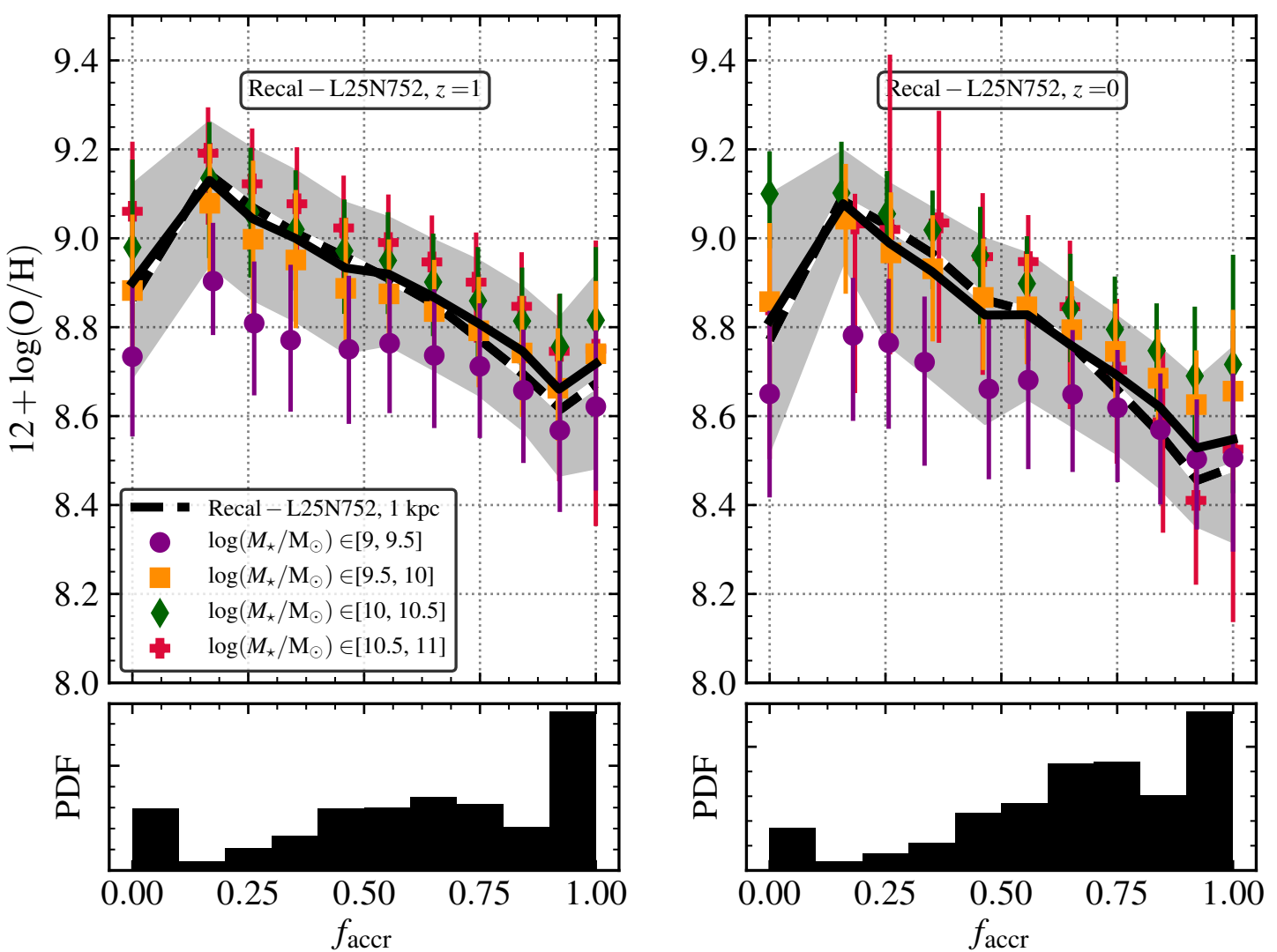

Figura G.1: Metalicidad de los spaxels en función de la fracción de gas acretado, $f_{\text {accr }}$, a redshift $z=0$ (paneles izquierdos) y $z=1$ (paneles derechos). La línea negra muestra los valores medios de la relación, mientras que la sombra gris denota la dispersión de $1 \sigma$ en percentiles 16 - 84. Los círculos violeta, cuadrado naranja, rombo verdes y signos "+" rosas denotan los valores medios de la relación para galaxias en determinado rango de masa estelar (como se muestra en la figura). Las barras de error corresponden a los percentiles $16-84$ de cada rango de masa estelar correspondiente. La línea negra a trazos representa la mediana de la relación para la simulación Recal-L25N752 utilizando spaxels de $1 \mathrm{kpc} \times 1 \mathrm{kpc}$. 